\title{
JUL 121990
}

Computer Programs for
Eddy-Current Defect Studies

Prepared by J. R. Pate, C. V. Dodd

Oak Ridge National Laboratory

Prepared for

U.S. Nuclear Regulatory Commission

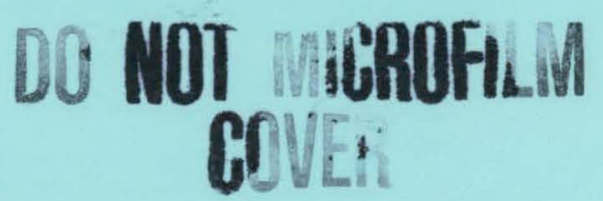




\section{DISCLAIMER}

This report was prepared as an account of work sponsored by an agency of the United States Government. Neither the United States Government nor any agency Thereof, nor any of their employees, makes any warranty, express or implied, or assumes any legal liability or responsibility for the accuracy, completeness, or usefulness of any information, apparatus, product, or process disclosed, or represents that its use would not infringe privately owned rights. Reference herein to any specific commercial product, process, or service by trade name, trademark, manufacturer, or otherwise does not necessarily constitute or imply its endorsement, recommendation, or favoring by the United States Government or any agency thereof. The views and opinions of authors expressed herein do not necessarily state or reflect those of the United States Government or any agency thereof. 


\section{DISCLAIMER}

Portions of this document may be illegible in electronic image products. Images are produced from the best available original document. 


\section{AVAILABILITY NOTICE}

Availability of Reference Materials Cited in NRC Publications

Most documents cited in NRC publications will be avallable from one of the following sources:

1. The NRC Public Document Room, 2120 L Street, NW, Lower Level, Washington, DC 20555

2. The Superintendent of Documents, U.S. Government Printing Office, P.O. Box 37082, Washington, DC 20013-7082

3. The National Technical Information Service, Springfield, VA 22161

Although the listing that follows represents the majority of documents clted in NRC publications, it is nut Intended to be exhaustive.

Rofcrenced documents avallable for inspection and copying for a fee from the NRC Public Document Room Include NRC correspondence and internal NRC memoranda; NRC Office of Inspection and Enforcement bulletins, circulars, information notices, Inspection and investigation notires; Licensee Event Reports; vendor reports and correspondence; Commission papers; and applicant and licensee documents and correspondence.

The following documents in the NUREG series are avallable for purchase from the GPO Sales Program: formal NRC staff and contractor reports, NRC-sponsored conference proceedings, and NRC booklets and brochures. Also available are Regulatory Guides, NRC regulations in the Code of Federal Regulations, and Nuclear Regulatory Commission Issuances.

Documents avallable from the National Technical Information Service Include NUREG series reports and technical reports prepared by other federal agencles and reports prepared by the Atomic Energy Commission, forerunner agency to the Nuclear Regulatory Commission.

Documents avallable from public and special technical libraries include all open literature items, such as books, Journal and periodical articles, and transactions. Federal Register notices, federal and state legislation, and congressional reports can usually be obtained from these llbrarles.

Documents such as theses, dissertations, foreign reports and translations, and non-NRC conference proceedings are avallable for purchase from the organization sponsuiliy the publlcation clted.

Single coples of NRC draft reports are avallable free, to the extent of supply, upon written request to the Office of Information Resources Management, Distribution Section, U.S. Nuclear Regulatory Commission, Washington, DC 20555.

Coples of industry codes and standards used in a substantive manner in the NRC regulatory process are maintained at the NRC Llbrary, 7920 Norfolk Avenue, Bethesda, Maryland, and are avallable there for reference use by the public. Codes and standards are usually copyrighted and may be purchased from the originating organization or, If they are American National Standards, from the American National Standards Institute, 1430 Broadway, New York, NY 10018.

\section{DISCLAIMER NOTICE}

This report was prepared as an accon unt of work sponsored by an agency of the Unilud States Government. Neither the United States Government nor any agency thereof, or any of their employees, makes any warranty, expresed or implied, or assumes any legal liability of responsibility for any third party's use, or the results of such use, of any information, apparatus, product or process disclosed in this report, or represents that its use by such third party would not infringe privately owned rights. 


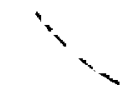

i NUREG/CR--5553

) $190013306^{3,5}$

I..

\section{Computer Programs for \\ Eddy-Current Defect Studies}

Manuscript Completed: March 1990

Date Published: June 1990

Prepared by

J. R. Pate, C. V. Dodd

Oak Ridge National Laboratory

Operated by Martin Marietta Energy Systems, Inc.

Dak Ridge National Laboratory

Oak Ridge, TN 37831

Prepared for

Division of Engineering

Otfice of Nuclear Regulatory Research

U.S. Nuclear Regulatory Commission

Washington, IX): 20555

NRC FIN B0417 


\section{THIS PAGE}

\section{WAS INTENTIONALLY \\ LEFT BLANK}


CONTENTS

INTRODUCTION . . . . . . . . . . . . . . . . . . . . . . . 1

PANCAKE COIL PROGRAMS . . . . . . . . . . . . . . . . . . . . . . . . . . 4

PCBLDF builds a magnitude and phase lookup file. . . . . . . 8

PCDSF calculates magnitude and phase of DSF for lattice of points . . . . . . . . . . . . . . . . . . . . . 17

PCDSFPLT generates a contour plot of magnitude of DSF . . . . 25

PCFIX converts raw data to normalized impedance change . . . 31

PCAVZSCN calculates defect impedance change, average over

PCAVVSCN calculates defect impedance change, average over

volume ....................... . 45

PCGRAPH plots two sets of data on same graph . . . . . . . . . . 59

PCINV inverts scan of pancake coil data to get depth and volume .. . . . . . . . . . . . . . . . . . . . . 64

PCRTSCAN converts raw voltage readings to impedance change. . 69

REFLECTION COIL PROGRAMS . . . . . . . . . . . . . . . . . . . . . . . . . 8.2

RFBLDF builds a lookup file of magnitude and phase of DSF . . 87

RFDSF calculates magnitude and phase of DSF for a lattice of

points . . . . . . . . . . . . . . . . . . . . 98

RFDSFPLT generates a contour plot of magnitude of DSF . . . . 107

RFAVZSCN calculates defect voltage change, average over

depth . . . . . . . . . . . . . . . . . . . . . . . . . 113

RFGRAPH plots two sets of data on same graph . . . . . . . . 131

RFINV inverts scan of defect using reflection coil data . . . 137

CIRCUMFERENTIAL BORESIDE COIL PROGRAMS . . . . . . . . . . . . . 143

ABBORAR calculates defect impedance change for absolute coil .. . . . . . . . . . . . . . . . . . . . . . 144

DBDSF calculates -DSF at lattice of points for differential coil...................... 159

DBDSFPLT generates a contour plot from DBDSF data . . . . . . 167

DFBORAR calculates defect impedance change, average over depth. . . . . . . . . . . . . . . . . . 173

CIRCUMFERENTIAL ENCIRCLING COIL PROGRAMS . . . . . . . . . . . . . . . $\quad .188$

ABENCAR calculates impedance change for absolute coil . . . . 189

DFENCAR calculates defect impedance change, average over depth................. 202

SUBROUTINES . . . . . . . . . . . . . . . . . . . . . . . . . . . . 218

$\operatorname{BESO}(\mathrm{XJOR}, \mathrm{R})$. . . . . . . . . . . . . . . . . . . . . . 219

$\operatorname{BESELI}(\mathrm{Q} 1, \mathrm{RJ} 1)$. . . . . . . . . . . . . . . . . . . . . . 220

$\operatorname{BESI}(\mathrm{X}, \mathrm{XIZRO}, \mathrm{XIONE})$. . . . . . . . . . . . . . . . . . . . . 221

$\operatorname{BESK}(\mathrm{X}, \mathrm{XKZRO}, \mathrm{XKONE})$. . . . . . . . . . . . . . . . . . 223 
$\operatorname{BESSEL}(\mathrm{XJ} 1, \mathrm{X}, \mathrm{R})$.

CMDBES (X, Y, ZIOR, ZIOI , ZKOR, ZKOI, BIIR, BIII, BKIR, BKII) . . . . . 226

GRID . . . . . . . . . . . . . . . . . . . . . . . . . 235

$\mathrm{IJBSSL}(\mathrm{A}, \mathrm{R} 1, \mathrm{R} 2, \mathrm{I}, \mathrm{J})$. . . . . . . . . . . . . . . . . 236

$\mathrm{KJBSSL}(\mathrm{A}, \mathrm{R} 1, \mathrm{R} 2, \mathrm{~K}, \mathrm{~J})$. . . . . . . . . . . . . . . . . . 239

PCLKUP (DEPTH, RHSMAG, LHSPHA) $\quad . \quad$. . . . . . . . . . . . . . . 243

RFLKUP(DEPTH, RHSMAG, LHSPHA) . . . . . . . . . . . . . . . . . 245

Data File REF.DAT . . . . . . . . . . . . . . . . . . . . 247

REFERENCES .... . . . . . . . . . . . . . . . . . . . . . . . . 248 
COMPUTER PROGRAMS FOR EDDY-CURRENT DEFECT STUDIES'

J. R. Pate and C. V. Dodd

\begin{abstract}
Several computer programs to aid in the design of eddycurrent tests and probes have been written. The programs, written in Fortran, deal in varlous ways with the response to defects exhibited by four types of probes: the pancake probe, the reflection probe, the circumferential boreside probe, and the circumferential encircling probe. Programs are included which calculate the impedance or voltage change in a coil due to a defect, which calculate and plot the defect sensitivity factor of a coil, and which invert calculated or experimental readings to obtain the size of a defect. The theory upon which the programs are based is the Burrows point defect theory, and thus the calculations of the programs will be more accurate for small defects.
\end{abstract}

\title{
INTRODUCTION
}

This report contains computer programs for a number of eddy-current probes applied to various test situations. The probes analyzed and presented here are the pancake probe, the reflection probe, the circumferential boreside probe, and the circumferential encircling probe. Both absolute and differential probes are used for the last two cases. The programs are written to run in Ryan-McFarland Fortran, although some of them have been run using Microsoft Fortran and NDP Fortran with very little change. The programs have been run on PC-AT clones, using either an Intel 80286 or 80386 microprocessor. Grafmatic plotting software from Microcompatibles is used for the contour plots and also must be installed on the machines.

The purpose of these programs is to analyze the effects of defects in conductors for the design of eddy-current tests, probes, and instrumentation. We can use them first to calculate the changes in eddy-

Research Sponsored by the Office of Nuclear Regulatory Research, Division of Engineering Safety, U. S. Nuclear Regulatory Commission under Interagency Agreement DOE 1886-8010-9B with the U.S. Department of Energy under contract DE-AC05-840R21400 with Martin Marietta Energy Systems, Inc. 
current readings that various types of defects produce. The defects can be treated as point defects, as point defects averaged over the depth of the actual defects, or as point defects averaged over the volume of the defects. The effect of a defect can also be inverted so that the volume and depth of the defect can be calculated from the instrument readings. In addition, we can plot the "defect sensitivity factor" (DSF) for the various probes and designs. The DSF at a point in a conductor is proportional to the coil voltage produced by a point defect at that location.

The programs were written and used for the analysis of eddy-current steam generator problems of interest to the Nuclear Regulatory Commission (NRC). Experimental measurements have been made that verify the accuracy of these programs for the NRC problems. However, they are quite general and may be used for a wide number of different eddy-current problems that have similar geometries. The programs are relatively fast running and sufficiently accurate so that eddy-current design studies of specific problems can be run with a minimum investment of time and equipment.

The accuracy of the impedance or voltage calculations for the cases without the defects is on the order of $0.005 \%$. This is more accurate than the coils and standards can be constructed. The accuracy of the impedance change produced by the defect depends in general on the size of the defect. In addition, several different methods for calculating the defect are given. The accuracy of the theory increases as the defect size decreases, but the experimental error increases. For the optimum defect size, the accuracy of the impedance change due to the defect is on the order of 20\%. However, most of the problem of eddy-current steam generator inspection is the elimination of the effect of unwanted property variations on the defect measurements, and the theory and programs listed here are well suited to study this problem.

This report is divided into five sections, one for each of the four coil types used and a fifth for common subroutines. We have attempted to make the description of each program sufficient (except for the subroutines), but it will probably be necessary to refer to an earlier prograll discussion in some cases. We also have given the equations that the programs are evaluating, along with the program variables used, so that the reader can more easily modify the programs for other uses.

The defect theory is based on the model of M. L. Burrows, with a correction of the sign of the term. Burrows gave an expression for both a "current defect" and a "magnetic defect," which only occurs in ferromagnetic materials. While the theory and discussion do carry the terms for ferromagnetic materials, the programs are written for nonferromagnetic materials, with a relative permeability of unity. None of the equations for the "magnetic defect" are given. The equation' for the voltage $V_{2 d}$ induced in coil 2 by the perturbation of the eddy-current flow from coil 1 due to the presence of a defect is: 


$$
\frac{V_{2 \mathrm{~d}}}{I_{1}}=-\left[\frac{3}{2} \sigma \omega^{2}\left(\frac{A_{2}}{I_{2}}\right)\left(\frac{A_{1}}{I_{1}}\right)\right] \times\left[\operatorname{Vol} \alpha_{22}\right]
$$

where $I_{1}$ and $I_{2}$ are the currents flowing in coil 1 and coil 2, respectively, and $A_{1}$ and $A_{2}$ are the vector potentials at the defect due to the currents flowing in the coils. The term $\sigma$ is the conductivity of the material, $\omega$ is the angular frequency, Vol is the volume of the defect and $\alpha_{22}$ is a shape and orientation factor for the defect. It is equal to unity for a spherical defect and assumed to be unity for all the other cases in this report.

The theory and equations are presented in this report only to aid in the explanation of the computer programs. A complete and rigorous derivation is left for other work. ${ }^{2-4}$ 
PANCAKE COIL PROGRAMS

The programs in this section perform various functions relating to the effect of a defect in a single conducting plate on the impedance of a pancake coil. Figure 1 shows a cutaway view of a pancake coil. In general, the shorter the coil, the greater its sensitivity to the property changes in the material. Thus, short flat coils shaped like a pancake, with a small relative inner diameter, are more sensitive to defects in the conductor. This is reasonable since the turns of wire are closer to the conductor than in a long narrow solenoid. However, the theory and programs work for any shape of coil and conductor with this geometry.

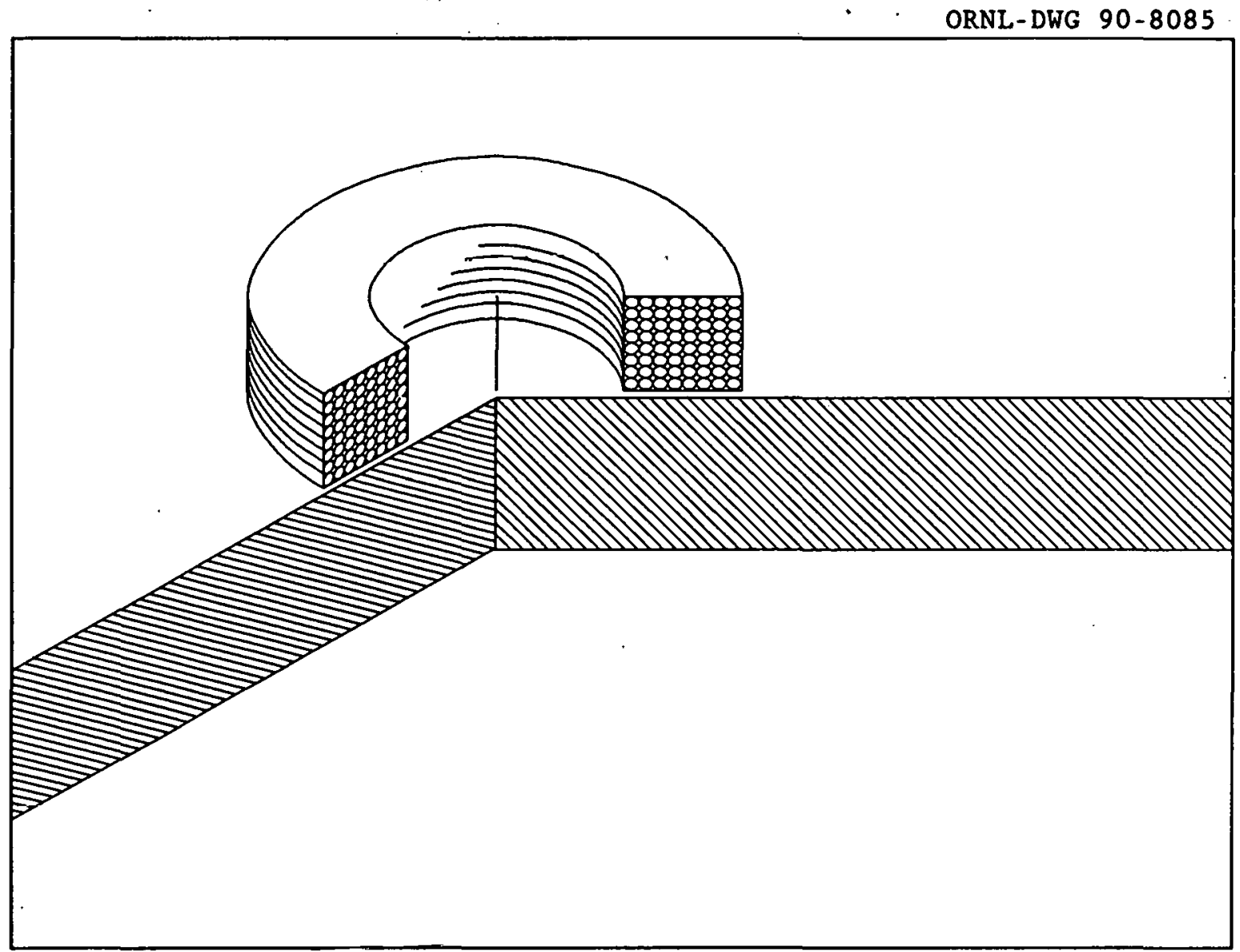

F1g. 1. Cutaway view of a pancake coil above a conducting plate.

Figure 2 shows a cross section of a pancake coil above a plate. The coil has been labeled with variables relating to its geometry which are common to all of the programs in this section. The coil is above a spherical defect, located at $r$ and $z$ with respect to the coil and the top surface of the plate, respectively.

The basic equations for the programs are presented below, with a detailed 


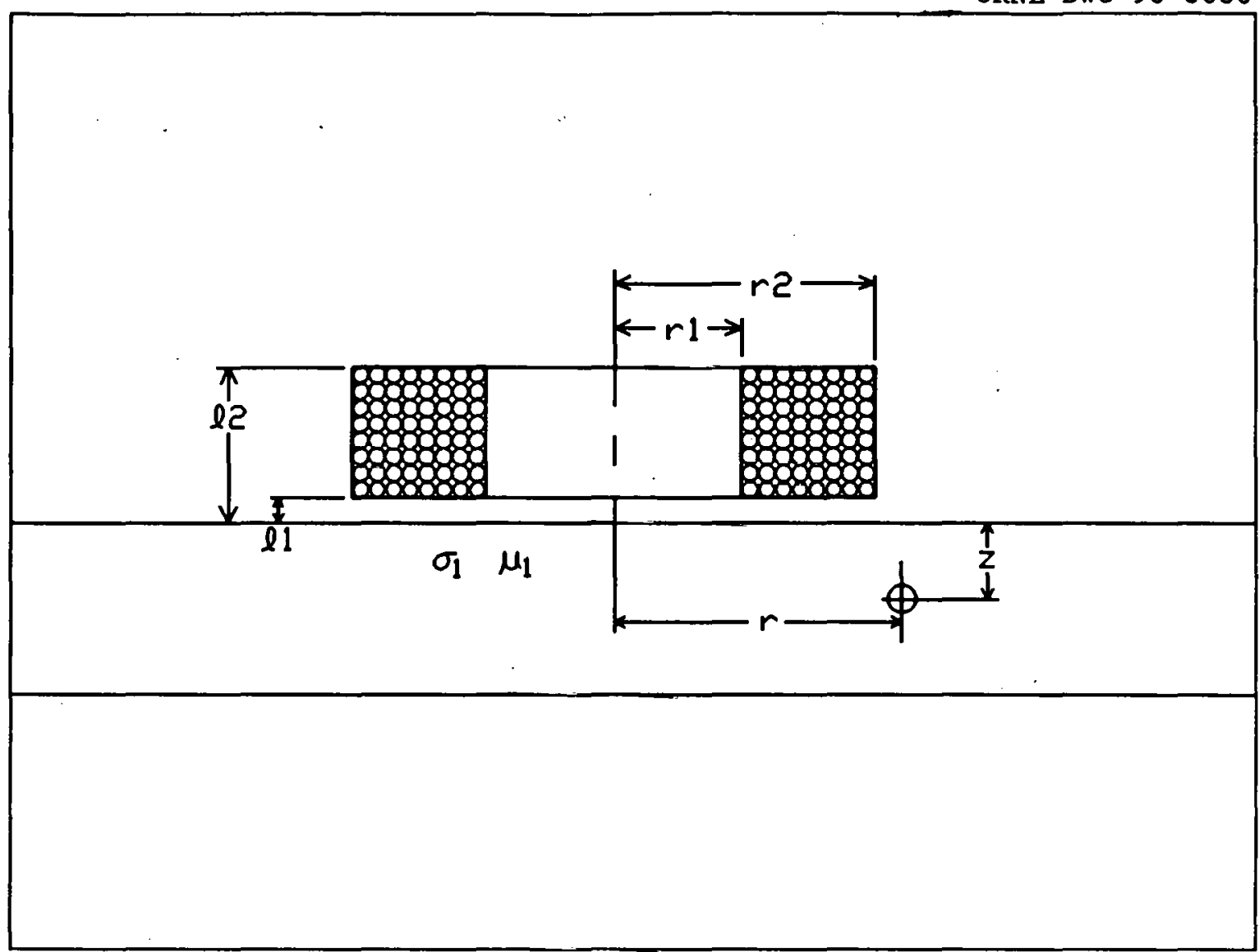

Fig. 2. Cross section of a pancake coil above a conducting plate.

derivation given elsewhere. The impedance change due to a small spherical defect at $r, z$ is:

$Z_{\mathrm{nd}}(r, z)=\frac{-3\left(\omega \mu \sigma, \overline{\mathrm{r}}^{2}\right)}{2 \pi I_{\mathrm{air}}} \operatorname{Vol}_{\mathrm{n}}\left[\int_{0}^{\infty} \frac{J\left(r_{2}, r_{1}\right) J_{1}(\alpha r)\left(e^{-\alpha \ell_{1}}-e^{-\alpha \ell_{2}} F\left(\alpha, \alpha_{1}, z\right)\right.}{\alpha^{3}} \mathrm{~d} \alpha\right]^{2}$

where $J\left(r_{2}, r_{1}\right)=\int_{\alpha r_{1}}^{q r_{2}} x J,(x) d x$

and $\quad \alpha_{1}=\left(\alpha^{2}+j \omega \mu \sigma_{1} \overline{\mathrm{r}}^{2}\right)^{1 / 2}$

The term $I_{\text {alr }}$ is related to the air inductance of the coil and is:

$I_{\mathrm{a} \mid r}=\int_{0}^{\infty} \frac{1}{\alpha^{b}}\left[J\left(r_{2}, r_{1}\right)\right]^{2} 2\left[\alpha\left(l_{2}-l_{1}\right)+\exp \left(-\alpha \ell_{2}+\alpha l_{1}\right)-1\right] d \alpha$ 
The term $F\left(\alpha, \alpha_{1}, z\right)$ depends on the number of planar layers of conductors. For the simple case of a semi-infinite plane beneath the coil we have:

$$
F\left(\alpha, \alpha_{1}, z\right)=\frac{\alpha e^{\alpha_{1} z}}{\alpha+\alpha_{1}}
$$

We can take the square root of both. sides of the equation, multiply by a weighting factor $r J_{1}(a r)$, and integrate over the signal produced as we scan across the defect:

$$
\begin{aligned}
& \int_{0}^{\infty} r J_{1}(\mathrm{ar}) \sqrt{-\mathrm{Z}_{\mathrm{nd}}(\mathrm{r}, z)} d r= \\
& {\left[\frac{3 \omega \mu \sigma, \bar{r}^{2}}{2 \pi I_{\mathrm{a} i r}} V o I_{\mathrm{n}}\right]^{1 / 2} \int_{0}^{\infty} r J_{1}(a r) d r \int_{0}^{\infty} \frac{J\left(r_{2}, r_{1}\right) J_{1}(\alpha r)\left(e^{-\alpha \ell_{1}}-e^{\left.-\alpha \ell_{2}\right) F\left(\alpha, \alpha_{1}, z\right)}\right.}{\alpha^{3}} d \alpha(6)}
\end{aligned}
$$

We shall now use the Fourier-Bessel Integral, which is:

$f(a)=\int_{0}^{\infty} r J_{1}(a r) \int_{0}^{\infty} \alpha J_{1}(\alpha r) f(\alpha) d \alpha d r$

to simplify the above equation. The result is:

$\int_{0}^{\infty} r J_{1}(\mathrm{ar}) \sqrt{-\mathrm{Z}_{\mathrm{nd}}(\mathrm{r}, \mathrm{z})} d r=\left[\frac{3 \omega \mu \sigma, \overline{\mathrm{r}}^{2}}{2 \pi I_{\mathrm{ar} r}} \mathrm{Vol}_{\mathrm{n}}\right]^{1 / 2} \frac{J\left(r_{2}, r_{1}\right)\left(e^{-a l_{1}}-e^{-a l_{2}} F\left(a, a_{1}, z\right)\right.}{a^{4}}$

We now transpose the equation and simplify the terms using some definitions:

$\sqrt{\operatorname{Vol}_{n}} e^{a_{1} z}=C M_{0} e^{i \theta}$

where

$C=\sqrt{\frac{2 \pi I_{\mathrm{a} 1 \mathrm{r}}}{3 \omega \mu \sigma_{1} \overline{\mathrm{r}}^{2}}} \frac{a^{3}}{J\left(r_{2}, r_{1}\right)\left[\exp \left(-a \ell_{1}\right)-\exp \left(-a \ell_{2}\right)\right]}$

$M_{0}=\operatorname{Mag}\left[\left(a+a_{1}\right) \int_{0}^{\infty} \sqrt{-Z_{n d}(r, z)} r J_{1}(a r) d r\right]$ 
and

$\theta=\operatorname{Pha}\left[\left(a+a_{1}\right) \int_{0}^{\infty} \sqrt{-Z_{n d}(r, z)} r J_{1}(a r) d r\right]$

Then the defect depth and volume can be calculated from the magnitude and phase shift of the expression and the real and imaginary parts of $a_{1}$, which we will call $x$ and $y$.

Thus

$$
z=\theta / y
$$

and

$$
V o I_{n}=\left[C M_{0} \exp (-x \theta / y)\right]^{2}
$$

For the simple case of the pancake coil above the semi-infinite conducting plate, we are able to directly invert the defect signal for a spherical defect and obtain the volume and depth of the defect. However, the equations become more messy for the case of a plate of thickness $c$. We generate functions that cannot be solved directly so we must use a lookup table. The program PCBLDF is used for this purpose and is discussed below. 
PCBLDF builds a magnitude and phase lookup file

Program PCBLDF builds a lookup file containing the magnitude and phase of the following integral

$$
\begin{aligned}
& \int_{0}^{\infty} \frac{1}{\alpha^{3}} J\left(r_{2}, r_{1}\right)\left[\frac{J_{0}\left(\alpha r_{1}\right)-J_{0}\left(\alpha r_{2}\right)}{\alpha}\right] \cdot\left[\exp \left(-\alpha \ell_{1}\right)-\exp \left(-\alpha \ell_{2}\right)\right] \\
& {\left[\frac{\alpha\left(\alpha_{1}+\alpha\right) \exp \left(\alpha_{1}(2 c+z)\right)+\alpha\left(\alpha_{1}-\alpha\right) \exp \left(-\alpha_{1} z\right)}{-\left(\alpha-\alpha_{1}\right)^{2}+\left(\alpha_{1}+\alpha\right)^{2} \exp \left(2 \alpha_{1} c\right)}\right] d \alpha}
\end{aligned}
$$

at different depths $z$ in a conducting plate. This is similar to the equation that we had for the semi-infinite plate except the term for $F\left(\alpha, \alpha_{1}, z\right)$ has been replaced by the term in the final set of large brackets. We have replaced the semi-infinite plate with a plate of thickness $c$. Also, we have used a weighting term so that the data used for the inversion are concentrated near the coil windings. This allows us to use data that have a higher signal-to-noise ratio. The weighting function is unity over the dimensions of the coil, from $r_{1}$ to $r_{2}$, and zero elsewhere. The result of this particular choice is that the function $J_{0}\left(\alpha r_{1}\right)-J_{0}\left(\alpha r_{2}\right)$ is produced.

This lookup file can be used by programs PCINV and PCRTSCAN to calculate the depth and volume of defects in the plate.

\section{Summary}

1. Dimension arrays and declare variable types.

2. Initialize variables.

3. Select a value for the depth in the plate at which to calculate the integral.

4. Calculate the integral.

5. Store the depth along with the magnitude and phase of the integral at this depth.

6. Loop to 3 until the calculations have been done at points all the way through the plate.

\section{$\underline{\text { Variables }}$}

Starred variables must be assigned by the user.

AIRIND $\quad$ The inductance in henries of the coil in air. 
FREQ $\quad$ The operating frequency in hertz.

$\mathrm{L}^{\circ}$

LI"

L2

LOD

LOU:

$\mathrm{MZT}^{\circ}$

NS

NZT

$\mathrm{R} 1^{\circ}$

R2

R3

RHSMAG

RHSPHA

RHO1 $^{\circ}$

$\mathrm{T} 1^{*}$

TRN $^{*}$

$\mathrm{U} 1^{\circ}$

WUSRR

2D

ZD2

ZMSTEP

The length of the coil. The value is input in inches and normalized by the program.

The lift-off of the coil. The vallue is input in inches and normalized by the program.

The normalized distance between the top of the coil and the plate. This value is: computed by the program.

The number of the I/O unit: connected to the output data file.

The number of the $I / O$ undt connected to the printer.

The number of depths throughout the plate at which the progran does the caliculations.

The side of the plate which is nearer to the point at which the integral. is being calculated. If NS $=1$, the point is closer to the near side; if NS $=2$, the point is closer to the far side.

The number of parts into which each defect is divided to perform the calculations.

The inner radius of the coil. The value is input in inches and normalized by the program.

The outer radius of the coil.. The value is input in inches and normalized by: the program.

The mean radius of the coiil in inches.

The magnitude of the integral' at a certain depth in the plate.

The phase in degrees of the integral at a certain depth in the plate.

The electrical resistivity of the plate in $\mu \Omega$ $\mathrm{cm}$.

The thickness of the plate.. The value is input in inches and normalized by the program.

The number of turns in the coili..

The relative magnetic permeabilitity of the plate. The product of the angular frequency, the magnetic permeability, the electrical conductivity, and the square of the mean coil radius.

The normalized distance from the side of the plate where the defect is located to the bottom of the defect. A negative number..

The normalized distance from the near side of the plate to the renter of the defect. A negative number.

The normalized axial distance between the depths at which the program does the calculations.

Notes

1. Program PCBLDF assists program PCINV in the inversion process. The programs use the equation 


$$
\begin{aligned}
& \int_{r_{1}}^{r_{2}}\left[-Z_{\mathrm{nd}}(r, z)\right]^{1 / 2} d r= \\
& {\left[\frac{3 \omega \mu \sigma \bar{r}^{2} V o I_{\mathrm{n}} \alpha_{22}}{2 \pi I_{\mathrm{air}}}\right]^{1 / 2}\left[\int_{0}^{\infty} \frac{1}{\alpha^{3}} J\left(r_{2}, r_{1}\right)\left[\frac{J_{0}\left(\alpha r_{1}\right)-J_{0}\left(\alpha r_{2}\right)}{\alpha}\right]\right.} \\
& \left.\left[\exp \left(-\alpha \ell_{1}\right)-\exp \left(-\alpha \ell_{2}\right)\right]\left[\frac{\alpha(\alpha+\alpha) \exp \left(\alpha_{1}(2 c+z)\right)+\alpha(\alpha,-\alpha) \exp \left(-\alpha_{1} z\right)}{-\left(\alpha-\alpha_{1}\right)^{2}+\left(\alpha_{1}+\alpha\right)^{2} \exp \left(2 \alpha_{1} c\right)}\right] d \alpha\right]
\end{aligned}
$$

Program PCBLDF calculates the integral on the righthand side of this equation. It is clear that since $V o I_{n}$, the defect volume, can be factored out of the right side of the equation, it has no effect on the phase of the right side of the equation. Therefore, the phase depends only upon $z$, the depth of the defect. So when program PCINV calculates the integral on the left-hand side, it obtains a value for the phase of this integral, and it can search the table built by program PCBLDF until it finds this phase. The depth in the table corresponding to this phase is the depth of the defect. Then, knowing the magnitude of both integrals (the magnitude of the integral on the right was calculated and stored in the file by program PCBLDF, and the magnitude of the integral was previously calculated by program PCINV), program PCINV can solve for the volume of the defect. 
Integration Section of Program PCBLDF

\section{Symbol definitions}

The following are definitions of the symbols used to describe the program variables appearing in the integration section of this program. All lengths are normalized unless otherwise noted.

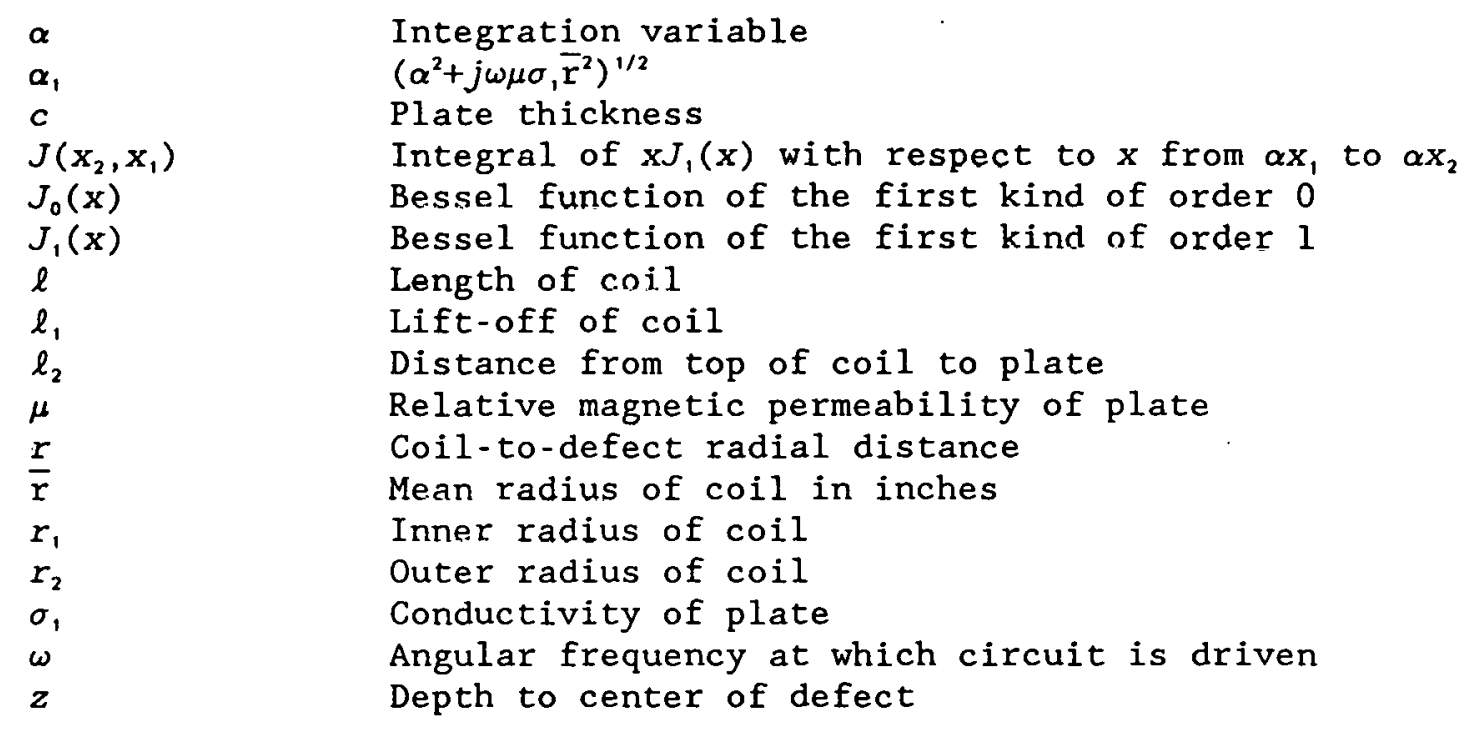

Variables appearing in the integration section

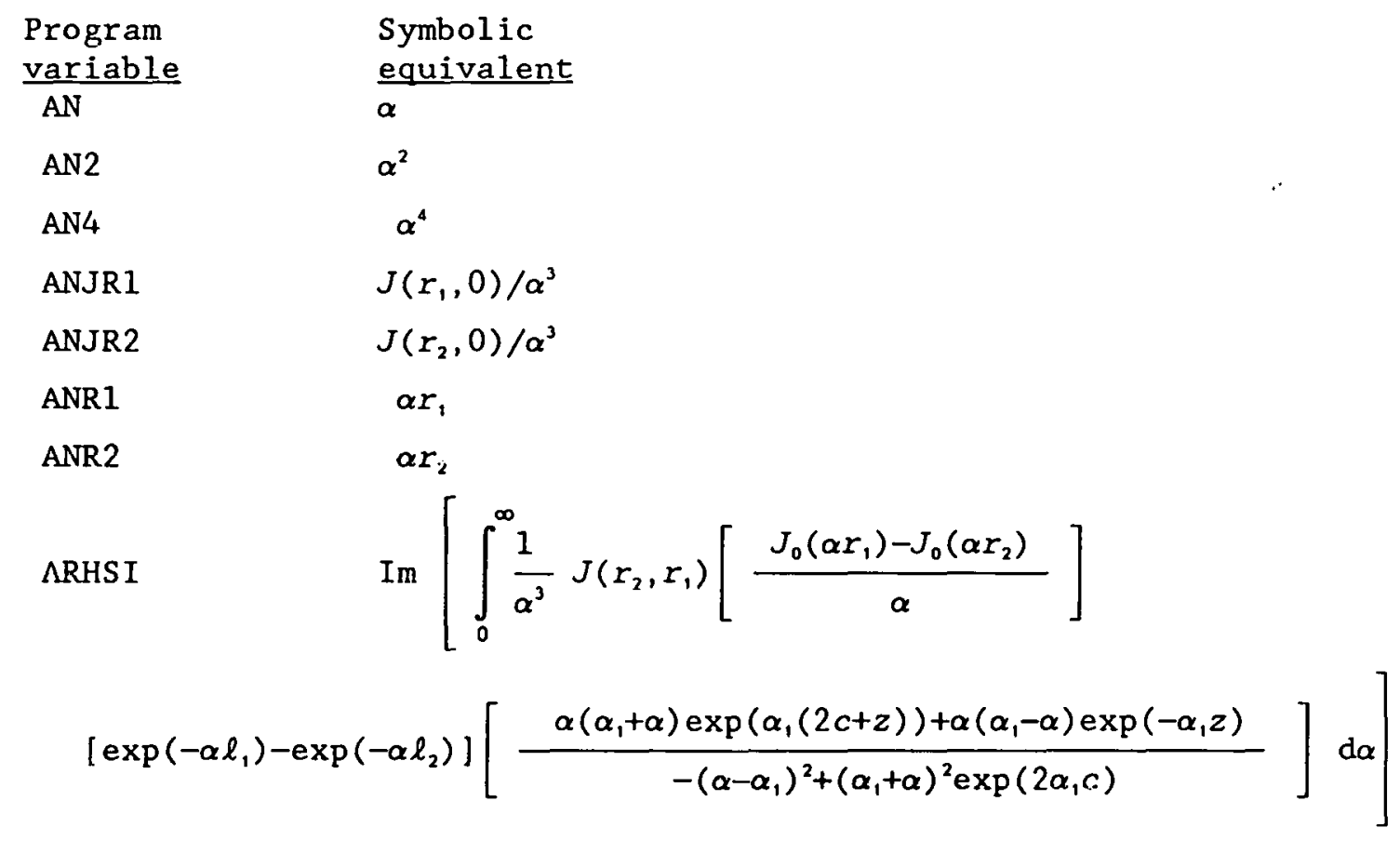


ARHSR $\quad \operatorname{Re}\left[\int_{0}^{\infty} \frac{1}{\alpha^{3}} J\left(r_{2}, r_{1}\right)\left[\frac{J_{0}\left(\alpha r_{1}\right)-J_{0}\left(\alpha r_{2}\right)}{\alpha}\right]\right.$
$\left.\left[\exp \left(-\alpha \ell_{1}\right)-\exp \left(-\alpha \ell_{2}\right)\right]\left[\frac{\alpha\left(\alpha_{1}+\alpha\right) \exp \left(\alpha_{1}(2 c+z)\right)+\alpha(\alpha,-\alpha) \exp (-\alpha, z)}{-\left(\alpha-\alpha_{1}\right)^{2}+\left(\alpha_{1}+\alpha\right)^{2} \exp (2 \alpha, c)}\right] \mathrm{d} \alpha\right]$

ASTP $\mathrm{d} \alpha$

DCOYNT1

$\operatorname{Re}[\exp (\operatorname{Im}(2 \alpha, c))]$

DCOYNZ

$\operatorname{Re}\left[\exp \left(\operatorname{Im}\left(\alpha_{1} z\right)\right)\right]$

DEXNT1

$\exp [\operatorname{Re}(2 \alpha, c)]$

DEXNZ

$\exp [\operatorname{Re}(\alpha, z)]$

DNI

$\operatorname{Im}\left[-\left(\alpha-\alpha_{1}\right)^{2}+(\alpha,+\alpha)^{2} \exp \left(2 \alpha_{1} c\right)\right]$

DNR

$\operatorname{Re}\left[-\left(\alpha-\alpha_{1}\right)^{2}+\left(\alpha_{1}+\alpha\right)^{2} \exp (2 \alpha, c)\right]$

DSIYNT1

$\operatorname{Im}[\exp (\operatorname{Im}(2 \alpha, c))]$

DSIYNZ

$\operatorname{Im}[\exp (\operatorname{Im}(\alpha, z))]$

FI

$\operatorname{Im}\left[\frac{\alpha\left(\alpha_{1}+\alpha\right) \exp (2 \alpha, c) \exp (\alpha, z)+\alpha(\alpha,-\alpha) \exp (-\alpha, z)}{-(\alpha-\alpha,)^{2}+(\alpha,+\alpha)^{2} \exp (2 \alpha, c)}\right]$

FR

$\operatorname{Re}\left[\frac{\alpha(\alpha,+\alpha) \exp (2 \alpha, c) \exp (\alpha, z)+\alpha(\alpha,-\alpha) \exp (-\alpha, z)}{-(\alpha-\alpha,)^{2}+(\alpha,+\alpha)^{2} \exp (2 \alpha, c)}\right]$

J01MJ02

$J_{0}\left(\alpha r_{1}\right)-J_{0}\left(\alpha r_{2}\right)$

JOR1

$J_{0}\left(\alpha r_{1}\right)$

JOR2

$J_{0}\left(\alpha r_{2}\right)$

JANR21

$J\left(r_{2}, r_{1}\right) / \alpha^{3}$

NMI

$\operatorname{Im}\left[\alpha(\alpha,+\alpha) \exp (2 \alpha, c) \exp \left(\alpha_{1} z\right)+\alpha(\alpha,-\alpha) \exp \left(-\alpha_{1} z\right)\right]$

NMI 1

$\operatorname{Im}[\alpha(\alpha,+\alpha) \exp (2 \alpha, c) \exp (\alpha, z)]$

NMI2

$\operatorname{Im}[\alpha(\alpha,-\alpha) \exp (-\alpha, z)]$

NMR

$\operatorname{Re}\left[\alpha(\alpha,+\alpha) \exp \left(2 \alpha_{1} c\right) \exp \left(\alpha_{1} z\right)+\alpha(\alpha,-\alpha) \exp \left(-\alpha_{1} z\right)\right]$

NMR 1

$\operatorname{Re}[\alpha(\alpha,+\alpha) \exp (2 \alpha, c) \exp (\alpha, z)]$

NMR2

$\operatorname{Re}[\alpha(\alpha,-\alpha) \exp (-\alpha, z)]$

RDEXNZ

$\exp [\operatorname{Re}(-\alpha, z)]$

RFAC

$$
\frac{1}{\alpha^{3}} J\left(r_{2}, r_{1}\right)\left[\frac{J_{0}\left(\alpha r_{1}\right)-J_{0}\left(\alpha r_{2}\right)}{\alpha}\right]\left[\exp \left(-\alpha \ell_{1}\right)-\exp \left(-\alpha \ell_{2}\right)\right] \mathrm{d} \alpha
$$




$$
\begin{aligned}
& \text { RHSI } \operatorname{Im}\left[\frac{1}{\alpha^{3}} \cdot T\left(r_{2}, r_{1}\right)\left[\frac{J_{0}\left(\alpha r_{1}\right)-J_{0}\left(\alpha r_{2}\right)}{\alpha}\right]\right. \\
& \left.\left[\exp \left(-\alpha l_{1}\right)-\exp \left(-\alpha l_{2}\right)\right]\left[\frac{\alpha\left(\alpha_{1}+\alpha\right) \exp \left(\alpha_{1}(2 c+z)\right)+\alpha(\alpha,-\alpha) \exp \left(-\alpha_{1} z\right)}{-\left(\alpha-\alpha_{1}\right)^{2}+(\alpha,+\alpha)^{2} \exp (2 \alpha, c)}\right] d \alpha\right] \\
& \operatorname{RHSR}\left[\frac{1}{\alpha^{3}} J\left(r_{2}, r_{1}\right)\left[\frac{J_{0}\left(\alpha r_{1}\right)-J_{0}\left(\alpha r_{2}\right)}{\alpha}\right]\right. \\
& \left.\left[\exp \left(-\alpha l_{1}\right)-\exp \left(-\alpha \ell_{2}\right)\right]\left[\frac{\alpha\left(\alpha_{1}+\alpha\right) \exp \left(\alpha_{1}(2 c+z)\right)+\alpha\left(\alpha_{1}-\alpha\right) \exp \left(-\alpha_{1} z\right)}{-\left(\alpha-\alpha_{1}\right)^{2}+\left(\alpha_{1}+\alpha\right)^{2} \exp \left(2 \alpha_{1} c\right)}\right] \mathrm{d} \alpha\right] \\
& \text { SMAIR } \quad \int_{0}^{\infty} \frac{2(\alpha \ell+\exp (-\alpha \ell)-1)\left[J\left(r_{2}, r_{1}\right)\right]^{2}}{\alpha^{6}} d \alpha \\
& \text { WUSRR2 }\left(\omega \mu \sigma, \overline{\mathrm{r}}^{2}\right)^{2} \\
& \mathrm{XN} \quad \operatorname{Re}\left[\alpha_{1}\right] \\
& \text { YN } \operatorname{Im}\left[\alpha_{1}\right]
\end{aligned}
$$

Notes for the integration section

I1. The program has been tested and found to be accurate enough with a step size of 0.01 and with the upper limit of the integration equal to 50 . 


\section{Listing}

PROGRAM PCBLDF
November 14, 1988
IMPLICIT REAL $* 8$ (A-H, O-Z)

REAL $* 8$ L, L1, L2, JANR21, LHSPHA

REAL *8 NMR1A, NMR1B , NMR1, NMR2, NMR

REAL *8 NMI1A, NMI1B, NMI1, NMI2, NMI

REAL*8 JOR1, JOR2, J01MJ02

DATA LOU/8/,LOD/40/,PI/3.141592653/

DATA FREQ/500./,RHO1/4.054/,U1/1.0/

DATA TRN $/ 800 . /, \mathrm{T} 1 / 0.25 /$, ASTP $/ 0.01 /$

C TIME AND DATE ARE PRINTED

CALL GETTIM( IHR, IMN, ISE, IFR)

CALL GETDAT (IYR, IMO, IDA)

IYR=IYR -1900

WRITE (LOU , 2) IHR, IMN, ISE, IMO, IDA, IYR

2 FORMAT(' PCBLDF TIME ', I2,' :', I2,' :', I2

$*$,' DATE ', I $2, \prime^{\prime}$, , I $2,{ }^{\prime}$, I 2 )

WRITE $($ LOU, 5$)$

5 FORMAT (6X, 'IN RAD', 4X, 'OT RAD', 4X, 'LENGTH' , $4 \mathrm{X}$, 'LIF OFF'

$*, 3 \mathrm{X},{ }^{\prime}$ CLADTH' )

$\mathrm{R} 1=0.1000$

$\mathrm{R} 2=0.4100$

$\mathrm{L}=0.1000$

$\mathrm{L} 1=0.01$

$\mathrm{R} \cdot 3=0.5 *(\mathrm{R} 1+\mathrm{R} 2)$

WRITE (LOU , 10) R1 , R2 , L , L1 , T1

$\mathrm{R} 1=\mathrm{R} 1 / \mathrm{R} 3$

$\mathrm{R} 2=2.0-\mathrm{R} 1$

$\mathrm{L}=\mathrm{L} / \mathrm{R} 3$

$\mathrm{L} 1=\mathrm{L} 1 / \mathrm{R} 3$

$\mathrm{T} 1=\mathrm{T} 1 / \mathrm{R} 3$

$\mathrm{L} 2=\mathrm{L}+\mathrm{L} 1$

WUSRR $=0.5093979 * \mathrm{U} 1 * \mathrm{R} 3 * \mathrm{R} 3 * \mathrm{FREQ} / \mathrm{RHO} 1$

WRITE(LOU , 15) R1, R2, L, L1, T1

10 FORMAT('ACT ', 5(F7.4,3X))

15. FORMAT ('NOR ', 5(F7.4,3X))

WRITE (LOU , 20)R3, FREQ , RHO1, U1, WUSRR

20 FORMAT (' RBAR',F7.4,' FREQ= ', 1PE13.6,' RHO=' , OPF9.4,

*' PERM=', F7.3,' WUSRR=' ,F9.4)

c:

SMAIR=AIRIND* $(L *(R 2-R 1)) * * 2 /(0.0254 * 4 . E-07 * T R N * T R N * R 3 * P I * P I)$

WUSRR $2=$ WUSRR $*$ WUSRR

ZMSTEP $=$ T1 $/$ MZT

DO $1200 \mathrm{MZ}=0, \mathrm{MZT}$

IF (MZ.GT . (0.5*MZT)) THEN

C Far side defect

NS $=2$

$\mathrm{ZM}=(\mathrm{MZT}-\mathrm{MZ}) * \mathrm{ZMSTEP}$ 


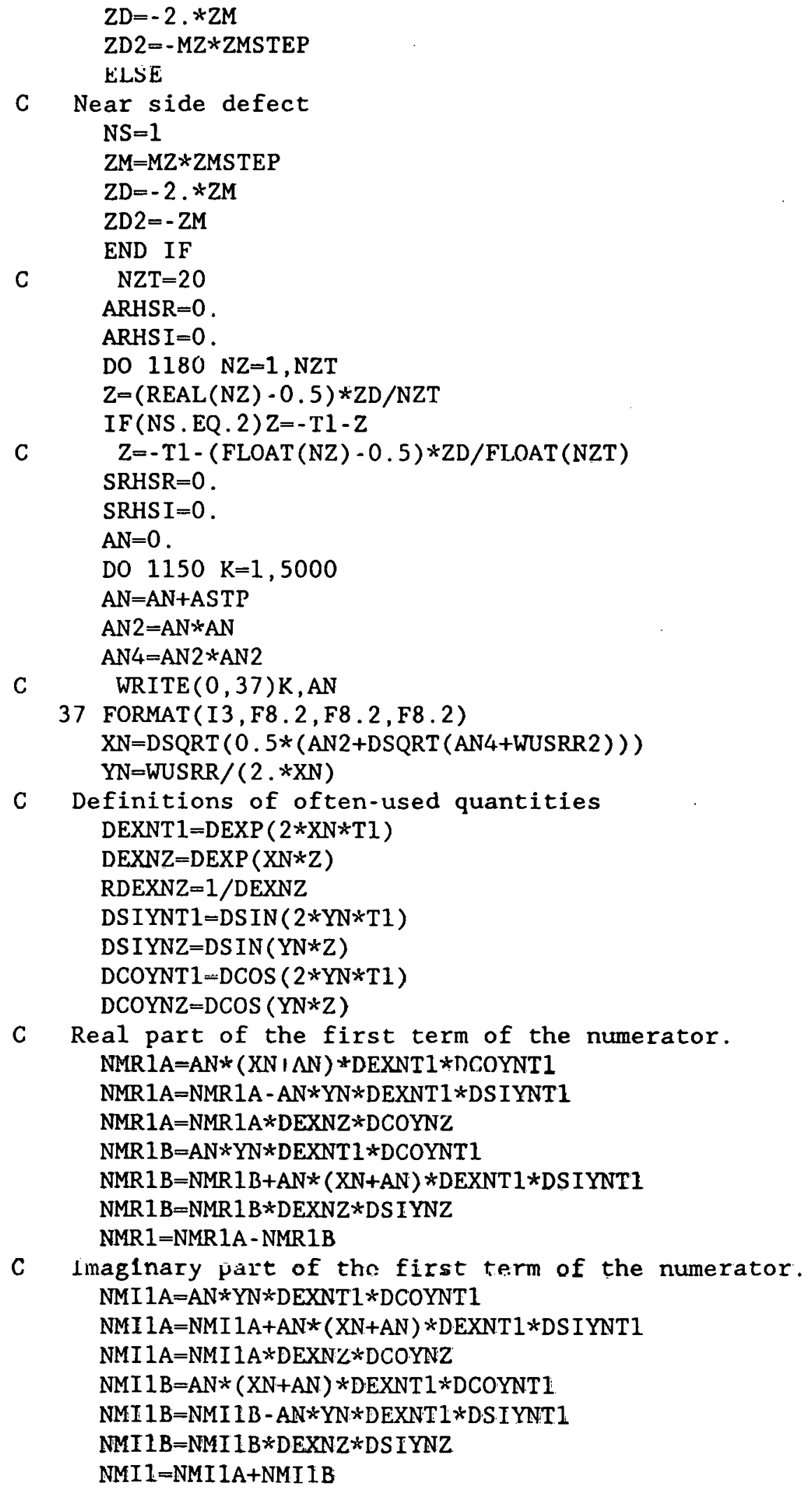


C Real part of the second term of the numerator. NMR $2=A N *(X N-A N) * R D E X N Z * D C O Y N Z$ NMR2 $=$ NMR $2+A N * Y N * R D E X N Z * D S I Y N Z$

C Imaginary part of the second term of the numerator. NMI $2=A N * Y N * R D E X N Z * D C O Y N Z$ NMI $2=$ NMI $2-A N *(X N-A N) * R D E X N Z * D S I Y N Z$

C Real part of the denominator. $\mathrm{DNR}=-(\mathrm{AN}-\mathrm{XN}) *(\mathrm{AN}-\mathrm{XN})+\mathrm{YN} * \mathrm{YN}$ $\mathrm{DNR}=\mathrm{DNR}+\mathrm{DEXNT} 1 *((\mathrm{XN}+\mathrm{AN}) *(\mathrm{XN}+\mathrm{AN})-(\mathrm{YN} * \mathrm{YN})) * \mathrm{DCOYNT} 1$ $D N R=D N R-D E X N T 1 *(2 * Y N *(X N+A N)) * D S I Y N T 1$

$C$ Imaginary part of the denominator. $\mathrm{DN} I=2 * \mathrm{YN} *(\mathrm{AN}-\mathrm{XN})$ DNI $=$ DNI + DEXNT $1 *(2 *(\mathrm{XN}+\mathrm{AN}) * \mathrm{YN}) * \mathrm{DCOYNT} 1$ $\mathrm{DNI}=\mathrm{DNI}+\mathrm{DEXNT} 1 *((\mathrm{XN}+\mathrm{AN}) *(\mathrm{XN}+\mathrm{AN})-\mathrm{YN} * \mathrm{YN}) * \mathrm{DSI} \mathrm{YNT} 1$

C

$\mathrm{NMR}=\mathrm{NMR} 1+\mathrm{NMR} 2$

$\mathrm{NMI}=\mathrm{NMI} 1+\mathrm{NMI} 2$

$\mathrm{FR}=(\mathrm{NMR} * \mathrm{DNR}+\mathrm{NMI} * \mathrm{DNI}) /(\mathrm{DNR} * \mathrm{DNR}+\mathrm{DNI} * \mathrm{DNI})$

$\mathrm{FI}=(\mathrm{NMI} * \mathrm{DNR}-\mathrm{NMR} * \mathrm{DNI}) /(\mathrm{DNR} * \mathrm{DNR}+\mathrm{DNI} * \mathrm{DNI})$

CALL BESSEL(ANJR2, AN, R2)

CALL BESSEL(ANJR1, AN, R1)

JANR2 $1=$ ANJ R2 - ANJR1

$\mathrm{ANR} 1=\mathrm{AN} * \mathrm{R} 1$

CALL BESO (JOR1, ANRI)

ANR2 $=A$ AN*R2

CALL BESO (J.OR2, ANR2)

JO1MJ 02 =JOR1-JOR2

RFAC $=$ JANR $21 * J 01 M J 02 *(\operatorname{DEXP}(-\operatorname{AN} * \mathrm{~L} 1)-\operatorname{DEXP}(-\mathrm{AN} * \mathrm{~L} 2)) * \mathrm{ASTP} / \mathrm{AN}$

$\mathrm{RHSR}=\mathrm{RFAC} * \mathrm{FR}$

RHSI $=$ RFAC*FI

C WRITE $(0,1149)$ AN, RHSR, RHSI

1149 FORMAT (F9.3 , 3X, D11.3, 3X, D11.3)

$S R H S R=S R H S R+R H S R$

SRHSI $=$ SRHS I +RHSI

1150 CONTINUE

ARHSR $=$ ARHSR+SRHSR

ARHSI=ARHS I+SRHS I

1180 CONTINUE

$\mathrm{ARHSR}=\mathrm{ARHSR} / \mathrm{NZT}$

ARHSI=ARHSI/NZT

RHSMAG $=$ DSQRT (ARHSR *ARHSR+ARHS I *ARHSI)

RHS PHA =ATAN2 (ARHSI , ARHSR) *180./PI

WRITE (LOD, 1205) ZD2, RHSMAG, RHSPHA

WRITE (LOU, 1205) ZD2, RHSMAG, RHSPHA

WRITE $(0,1205)$ ZD2, RHSMAG , RHSPHA

1200 CONTINUE

1205 FORMAT (F6.3 1X, D11. 3, 1X, F7 . 2)

STOP

END 
PCDSF calculates mag. and phase of DSF for lattice of points

Program PCDSF calculates the magnitude and phase of the defect sensitivity factor of a pancake coil at a lattice of points throughout a plate. It stores the calculations so that they can be plotted by program PCDSFPLT. In Fig. 3 we show a pancake coil above a conducting plate, with the plate divided into a lattice of points.

ORNL-DWG $90-8087$

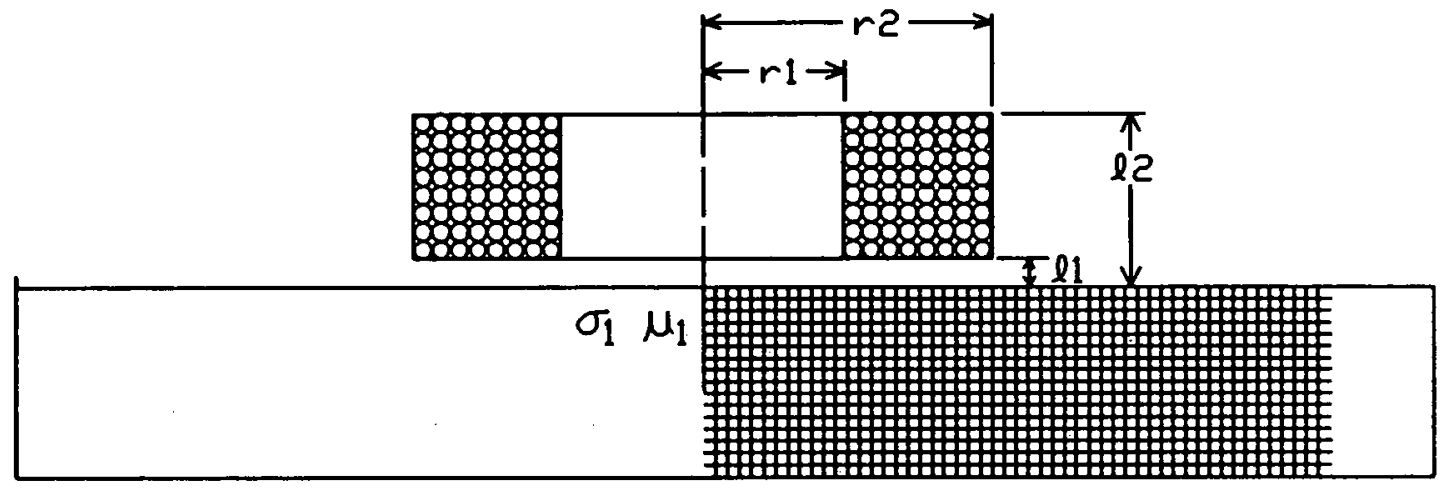

Fig. 3. Pancake coil with the conductor divided into a lattice of points.

The defect sensitivity factor for the pancake coil above a conducting plate at a point $r, z$ in the plate is:

$D S F(r, z)=\frac{-3\left(\omega \mu \sigma, \overrightarrow{\mathrm{r}}^{2}\right)}{2 \pi I_{\mathrm{air} .}}\left[\int \frac{J\left(\mathrm{r}_{2}, \mathrm{r}_{1}\right) J_{1}(\alpha r)\left(e^{-\alpha \ell_{1}}-e^{-\alpha \ell_{2}} F\left(\alpha, \alpha_{1}, z\right)\right.}{\alpha^{3}} \mathrm{~d} \alpha\right]^{2}$

The various terms in the equation are explained in Eqs. (2), (3), (4), and (15). Since the points to be calculated are in a regular lattice, and the terms in the numerical integration are factorable, a considerable amount of computation time can be saved by performing the computations that vary 
over the $r$ dimension and the $z$ dimension separately for each value of $\alpha$. The values of the different factors that depend only on $r$ or $z$ are computed once and stored in an array. Then for the different locations the array values are multiplied and then summed to compute the integral. The output from this program is stored in the data file PCDSF.DAT.

\section{Summary}

1. Dimension arrays and declare variable types.

2. Initialize variables.

3. Write the information about the coil and plate geometry to a file.

4. Do the parts of the integration which are independent of the position of the point.

5. Choose a value for the axial coordinate of the position of the point. Do the parts of the integration that depend only upon this coordinate.

6. Choose a value for the radial coordinate of the position of the point. Complete the integration for this point.

7. Loop to 6 until done.

8. Loop to 5 until done.

9. Write the results to a data file.

\section{Variables}

DELTAR ${ }^{\circ}$ The normalized radial distance between adjacent points at which the program calculates the defect sensitivity factor.

DELTAZ The normalized axial distance between adjacent points at which the program calculates the defect sensitivity factor.

DSFI The imaginary part of the defect sensitivity factor.

DSFM The magnitude of the defect sensitivity factor.

DSFP The phase in radians of the defect sensitivity factor.

DSFR The real part of the defect sensitivity factor.

FREQ $\quad$ The operating frequency in hertz.

$\mathrm{L}^{\circ} \quad$ The length of the coil. The value is input in inches and normalized by the program

L1 The lift-off of the coil. The value is input in inches and normalized by the program.

LOD $\quad$ The number of the I/O unit connected to the output data file.

LOU The number of the $I / O$ unit connected to the printer.

NRT' The total number of points in the radial direction at which the defect sensitivity factor is to be calculated.

NZT' The total number of points in the axial direction at which the defect sensitivity factor is to be calculated.

$\mathrm{R}^{\circ}$ The inner radius of the coil. The value is input 


\begin{tabular}{|c|c|}
\hline $\mathrm{R}^{\circ}$ & $\begin{array}{l}\text { in inches and normalized by the program: } \\
\text { The outer radius of the coil. The value is input } \\
\text { in inches and normalized by the program. }\end{array}$ \\
\hline R3 & The mean radius of the coil in inches. \\
\hline RD & $\begin{array}{l}\text { The normalized radial distance between the coil } \\
\text { axis and the point at which the program is } \\
\text { calculating the defect sensitivity factor. }\end{array}$ \\
\hline $\begin{array}{l}\mathrm{RHO}^{\circ} \\
\mathrm{T} 1^{\circ}\end{array}$ & $\begin{array}{l}\text { The resistivity of the plate in } \mu \Omega-\mathrm{cm} \text {. } \\
\text { The thickness of the plate. The value is input } \\
\text { in inches and normalized by the program. }\end{array}$ \\
\hline TRN" & The number of turns in the coil. \\
\hline $\mathrm{U} 1^{\circ}$ & The relative magnetic permeability of the plate. \\
\hline WUSRR & $\begin{array}{l}\text { The product of the angular operating frequency, } \\
\text { the magnetic permeability of the plate, the } \\
\text { electrical conductivity of the plate, and the } \\
\text { square of the mean radius of the coil. }\end{array}$ \\
\hline
\end{tabular}

Notes

1. The integration in this program is very similar to the integration in program PCAVZSCN. Program PCAVZSCN calculates the impedance change in a pancake coil due to a defect, but in order to do this, it must calculate the defect sensitivity factor. The main difference between the programs is that program PCDSF calculates the defect sensitivity factor at different depths in the plate while program PCAVZSCN calculates the average defect sensitivity factor over a range of depths.

Sample output

Output sent to printer:

$\begin{array}{llllll}\text { PCDSF } & \text { TIME } & 7: 44: 26 & \text { DATE } 8 / 9 / 89 & \\ & \text { IN RAD } & \text { OT RAD } & \text { LENGTH } & \text { LIFTOFF } & \text { CLADTH } \\ \text { ACT } & 0.1000 & 0.4250 & 0.0500 & 0.0100 & 0.2500 \\ \text { NOR } & 0.3810 & 1.6190 & 0.1905 & 0.0381 & 0.9524\end{array}$

RBAR 0.2625 FREQ $=6.000000 E+02$ RHO=4.0540 PERM $=1.000$ WUSRR $=5.1950$

NORM IMPD:RL 0.190682 IM 0.777969 AIR IND $1.099840 \mathrm{E}-02$

Partial 1isting of output stored on PCDSF.DAT:

\begin{tabular}{lrrr}
\multicolumn{2}{l}{40} & \multicolumn{1}{l}{40} \\
0.05000 & 0.02442 & \\
0.38095 & 1.61905 & \\
0.19048 & 0.03810 & \\
0.95238 & & \\
1 & 1 & $0.17513 \mathrm{D}-03$ & $0.24106 \mathrm{D}+01$ \\
1 & 2 & $0.16525 \mathrm{D}-03$ & $0.23596 \mathrm{D}+01$ \\
1 & 3 & $0.15496 \mathrm{D}-03$ & $0.23083 \mathrm{D}+01$ \\
1 & 4 & $0.14450 \mathrm{D}-03$ & $0.22563 \mathrm{D}+01$ \\
1 & 5 & $0.13407 \mathrm{D}-03$ & $0.22037 \mathrm{D}+01$ \\
1 & 6 & $0.12385 \mathrm{D}-03$ & $0.21504 \mathrm{D}+01$
\end{tabular}


$\begin{array}{llll}1 & 7 & 0.11397 D-03 & 0.20963 D+01\end{array}$

$\begin{array}{llll}1 & 8 & 0.10454 D-03 & 0.20413 D+01\end{array}$

$\begin{array}{llll}1 & 9 & 0.95618 \mathrm{D}-04 & 0.19856 \mathrm{D}+01\end{array}$

$110 \quad 0.87259 \mathrm{D}-04 \quad 0.19292 \mathrm{D}+01$ 


\section{Listing}

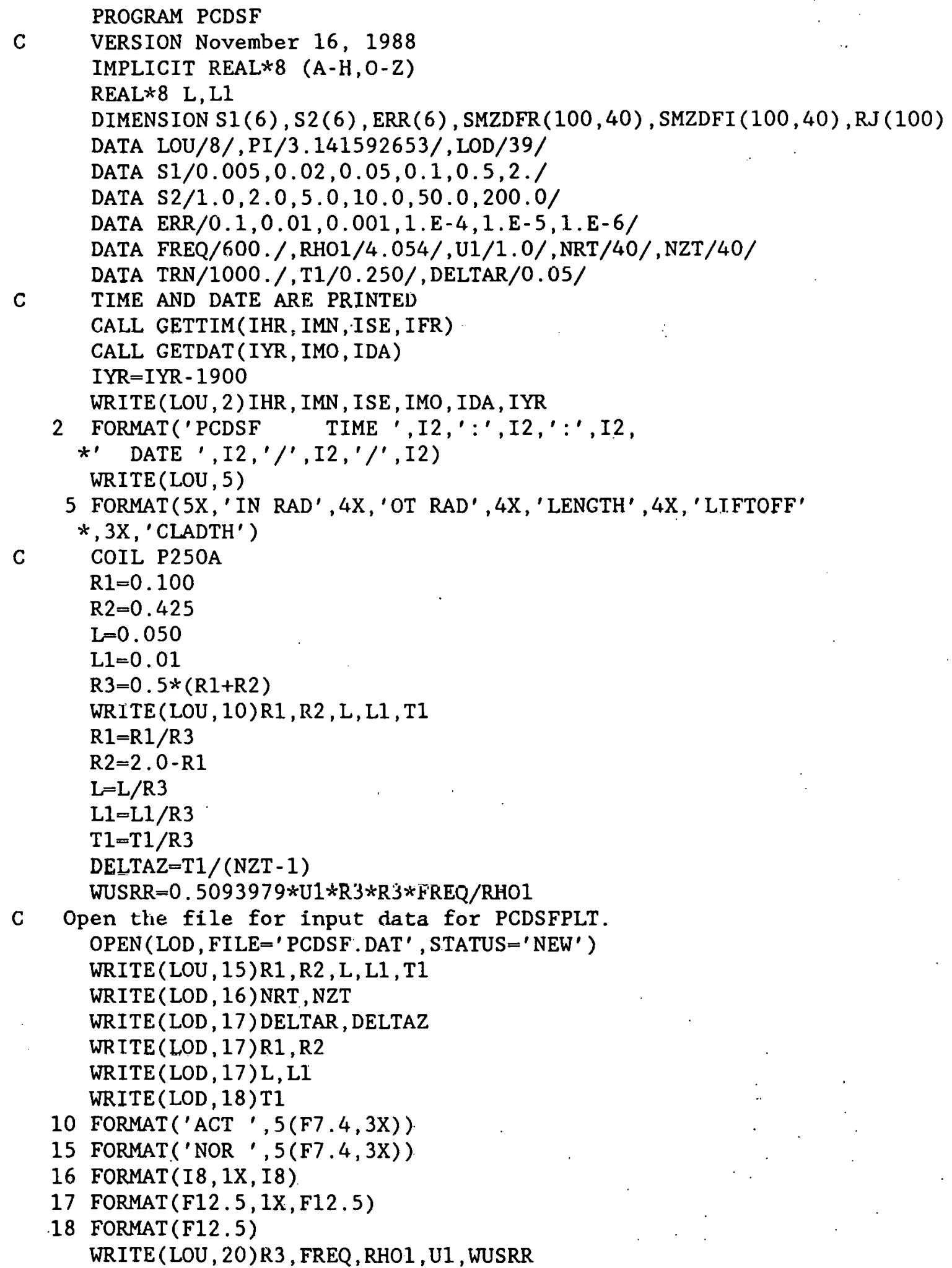




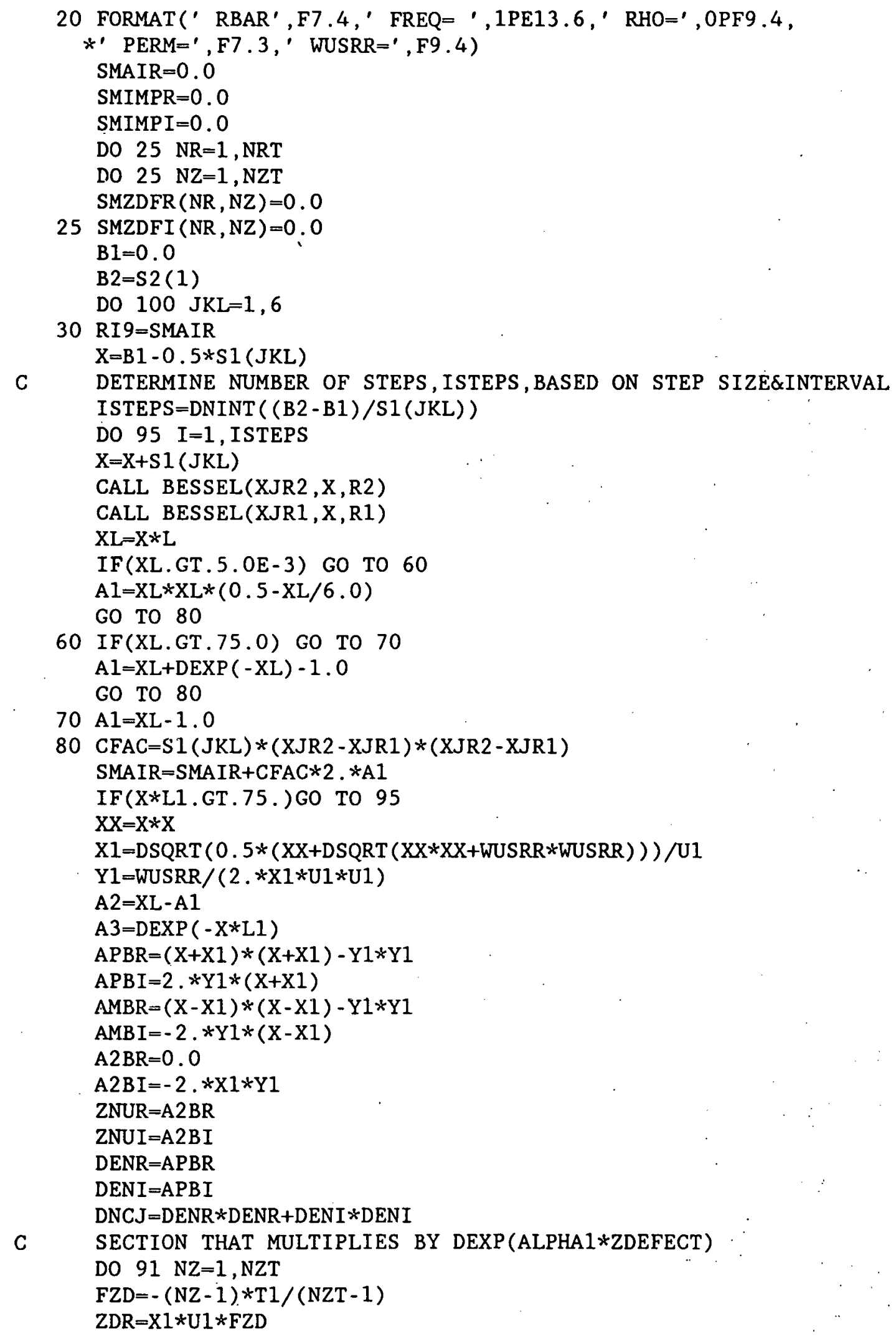


IF(ZDR.LT . -60.0)GO TO 93

$\mathrm{ZDI}=\mathrm{Y} 1 * \mathrm{U} 1 * \mathrm{FZD}$

$X P D R=D E X P(Z D R)$

$\mathrm{CSDI}=\mathrm{DCOS}(\mathrm{ZDI}) * \mathrm{XPDR}$

SNDI $=D S I N(2 D I) * X P D R$

$\mathrm{XX} 1=\mathrm{X} * \mathrm{X} 1+\mathrm{XX}$

$\mathrm{XY} 1=\mathrm{X} * \mathrm{Y} 1$

$\mathrm{X} 1 \mathrm{X}=\mathrm{X} * \mathrm{X} 1-\mathrm{XX}$

$\mathrm{ZNDR}=\mathrm{XX} 1 * \mathrm{CSDI}-\mathrm{XY} 1 *$ SNDI

$\mathrm{ZNDI}-\mathrm{XXI} * \mathrm{SNDI}+\mathrm{XY} 1 * \mathrm{CSDI}$

C SECTION THAT MULTIPLIES BY DEXP(ALPHA $*(2 * T H+Z D E F E C T))$

$\mathrm{TZR}=\mathrm{X} 1 * \mathrm{U} 1 *(2 . * \mathrm{~T} 1+\mathrm{FZD})$

IF(TZR.GT.60.) GO TO 87

$\mathrm{TZI}=\mathrm{Y} 1 * \mathrm{U} 1 *(2 . * \mathrm{~T} 1+\mathrm{FZD})$

$X P Z R=D E X P(-T Z R)$

$\mathrm{CSZI}=\mathrm{DCOS}(\mathrm{TZI}) * X P Z R$

$S N Z I=D S I N(T Z I) * X P Z R$

$\mathrm{ZNDR}=\mathrm{XX} 1 * \mathrm{CSDI}-\mathrm{XY} 1 * \mathrm{SNDI}+\mathrm{X} 1 \mathrm{X} * \mathrm{CSZI}+\mathrm{XY} 1 * \mathrm{SNZI}$

$\mathrm{ZNDI}=\mathrm{XX} 1 * \mathrm{SNDI}+\mathrm{XY} 1 * \mathrm{CSDI}+\mathrm{XY} 1 * \mathrm{CSZI}-\mathrm{X} 1 \mathrm{X} * \mathrm{SNZI}$

C SECTION THAT MULTIPLIES BY DEXP (-ALPHA $1 * 2 *$ CLADTH)

$\mathrm{TR}=2 . * \mathrm{X} 1 * \mathrm{U} 1 * \mathrm{~T} 1$

IF (TR.GT.60.)GO TO 87

$\mathrm{TI}=2 . * \mathrm{Y} 1 * \mathrm{U} 1 * \mathrm{~T} 1$

$\mathrm{XPTR}=\mathrm{DEXP}(-\mathrm{TR})$

$\operatorname{CSTI}=\mathrm{DCOS}(\mathrm{TI}) * \mathrm{XPTR}$

$\operatorname{SNTI}=\mathrm{DSIN}(\mathrm{TI}) * X P T R$

$\mathrm{DENR}=\mathrm{APBR}-\mathrm{AMBR} * \mathrm{CSTI}-\mathrm{AMBI} * \mathrm{SNTI}$

$\mathrm{DENI}=\mathrm{APBI}-\mathrm{AMBI} * \mathrm{CSTI}+\mathrm{AMBR} * \mathrm{SNTI}$

$\mathrm{ZNUR}=\mathrm{A} 2 \mathrm{BR}-\mathrm{A} 2 \mathrm{BR} * \mathrm{CSTI}-\mathrm{A} 2 \mathrm{BI} * \mathrm{SNTI}$

$\mathrm{ZNUI}=\mathrm{A} 2 \mathrm{BI}-\mathrm{A} 2 \mathrm{BI} * \mathrm{CSTI}+\mathrm{A} 2 \mathrm{BR} * \mathrm{SNTI}$

$\mathrm{DNCJ}=\mathrm{DENR} * \mathrm{DENR}+\mathrm{DENI} * \mathrm{DENI}$

$87 \mathrm{ZDRL}=(\mathrm{ZNDR} * \mathrm{DENR}+\mathrm{ZNDI} * \mathrm{DENI}) / \mathrm{DNCJ}$

$\mathrm{ZDIM}=(\mathrm{DENR} * \mathrm{ZNDI}-\mathrm{ZNDR} * \mathrm{DENI}) / \mathrm{DNCJ}$

$\mathrm{DFAC}=\mathrm{A} 2 * \mathrm{~A} 3 * \mathrm{~S} 1(\mathrm{JKL}) *(\mathrm{XJR} 2-\mathrm{XJR} 1)$

C

LOOP OVER THE R VARIATION FOR THE DEFECT

DO YU NK=1, NRT

IF (NZ.GT.1)GO TO 89

$\mathrm{RD}=($ FLOAT $(\mathrm{NR})-.5) * \mathrm{DELTAR}$

$\mathrm{XRD}=\mathrm{X} * \mathrm{RD}$

CALL BESEL1 (XRD, RJ1)

$\mathrm{RJ}(\mathrm{NR})=\mathrm{RJ} 1$

$89 \operatorname{SMZDFR}(\mathrm{NR}, \mathrm{NZ})=\operatorname{SMZDFR}(\mathrm{NR}, \mathrm{NZ})+\mathrm{RJ}(\mathrm{NR}) * \mathrm{ZDRL} * \mathrm{DFAC}$

$90 \operatorname{SMZDFI}(\mathrm{NR}, \mathrm{NZ})=\operatorname{SMZDFI}(\mathrm{NR}, \mathrm{NZ})+\mathrm{RJ}(\mathrm{NR}) * \mathrm{ZDIM} * \mathrm{DF} \Lambda \mathrm{C}$

91 CONTINUE

$93 \mathrm{ZRL}=(\mathrm{ZNUR} * \mathrm{DENR}+\mathrm{ZNUI} * \mathrm{DENI}) / \mathrm{DNCJ}$

$\mathrm{ZIM}=(\mathrm{DENR} * \mathrm{ZNUI}-\mathrm{ZNUR} * \mathrm{DENI}) / \mathrm{DNCJ}$

SMIMPR $=$ SMIMPR $+\mathrm{A} 2 * \mathrm{~A} 2 * \mathrm{~A} 3 * \mathrm{~A} 3 * \mathrm{ZRL} * \mathrm{CFAC}$

SMIMPI $=$ SMIMP $I+A 2 * A 2 * A 3 * A 3 * 2 I M * C F A C$

95 CONTINUE

$\mathrm{B} 1=\mathrm{B} 2$

$\mathrm{B} 2=\mathrm{B} 2+\mathrm{B} 2(\mathrm{JKL})$ 


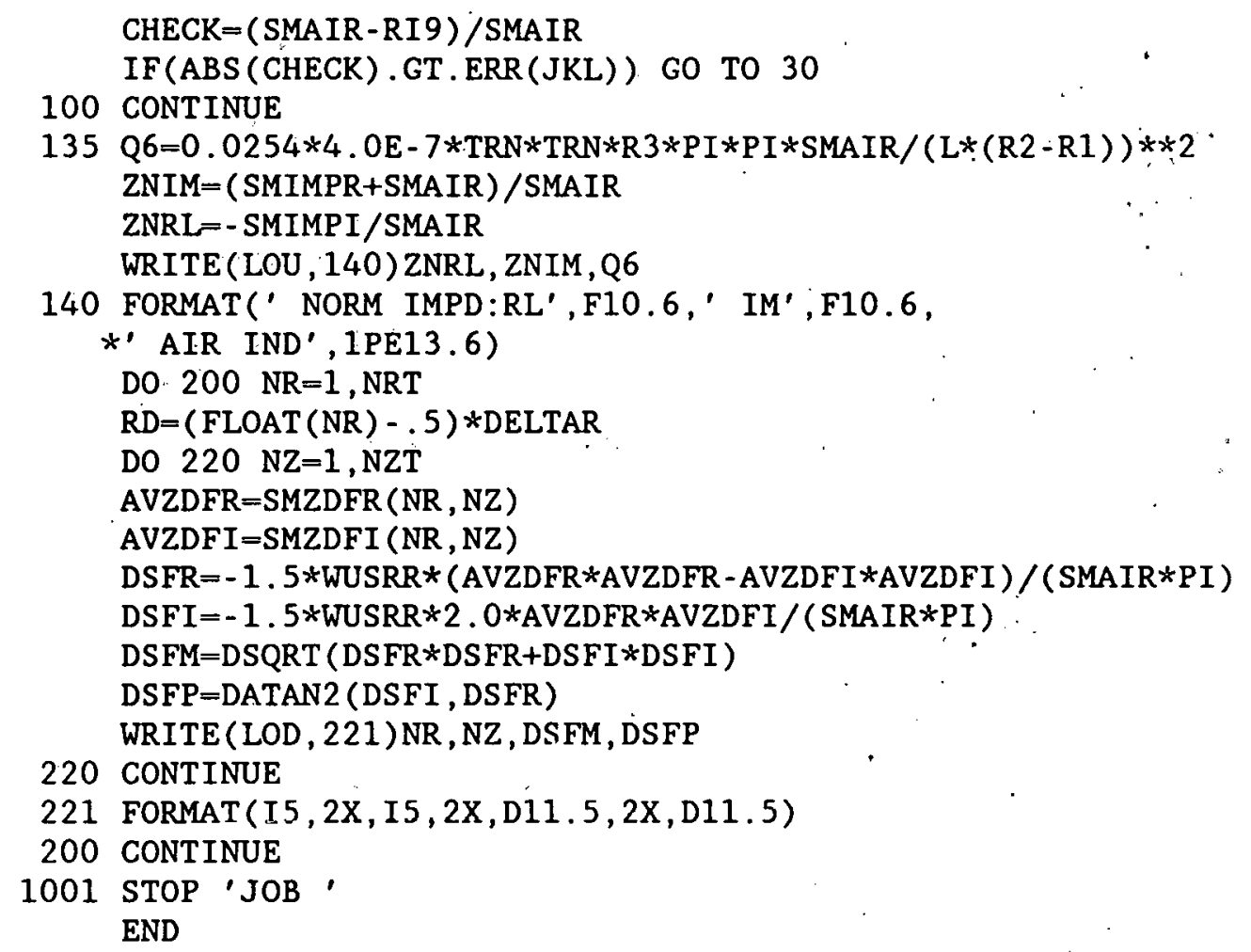


PCDSFPLT generates a contour plot of magnitude of DSF

Program PCDSFPLT generates a contour plot of the magnitude of the defect sensitivity factor for a pancake coil using calculations performed and stored by program PCDSF. The cross section of the coil and the coil axis are also drawn on the plot with the same scale as the contours, to show their relative positions. Only a small change in the program is necessary to compute and plot contours of the phase of the defect sensitivity factor. There are two lines with the label 140, and one is commented out. With the first line in, the magnitude contours are plotted, and with the second line the phase contours are plotted. Both use the same data file from PCDSF.

\section{Summary}

1. Dimension arrays and declare variable types.

2. Initialize variables.

3. Open the file created by program PCDSF.

4. Read in the information about the coil and the plate from the file.

5. Calculate the position of the data points in the normalized coordinate system.

6. Set the label flags for the contours.

7. Read the data stored by program PCDSF into array DSFMA.

8. Specify the values of the magnitude of the defect sensitivity factor where the contours are to be drawn.

9. Call the PRINTMATIC contour initialization routines.

10. Draw the contours.

11. Draw the coil and the plate.

\section{Variables}

Starred variables must be set by the user.

$\mathrm{CNM}^{\circ} \quad$ Array giving the values of the magnitude of the defect sensitivity factor at which contours are to be drawn.

DELTAR The normalized distance in the radial direction between adjacent data points.

DELTAZ The normalized distance in the axial direction between adjacent data points.

DSFMA Array containing the values of the magnitude of the defect sensitivity factor which were read in from the data file.

DSFMMAX The maximum value of the magnitude of the defect sensitivity factor.

DSFMMIN The minimum value of the magnitude of the defect sensitivity factor.

L The normalized length of the coil.

L1 The normalized lift-off of the coil.

LBM ${ }^{\circ} \quad$ Array which tells the program which of the 
contours are to be labeled with their values. If all elements of LBM are zero, none of the contours will be labeled.

LOE $\quad$ The number of the I/O unit connected to the input data file.

NAME' Character variable which contains the name of the file which this program uses for output.

NC $C^{*} \quad$ The number of contours to be drawn. This number must be less than or equal to 10 .

NRT The total number of points in the radial direction at which calculations were performed.

NZT The total number of points in the axial direction at which calculations were performed.

R1 The normalized inner coil radius.

R2 The normalized outer coil radius.

T1 The normalized thickness of the plate.

$\mathrm{XX} \quad$ Array describing the radial location of the data points in the normalized coordinate system.

$Y Y \quad$ Array describing the axial location of the data points in the normalized coordinate system.

\section{Notes}

1. The coordinate system set up and used by this program has its origin at the intersection of the coil axis and the near side of the plate. One unit of distance in the coordinate system is equal to one mean radius of the driver coil.

2. The array DSFMA must be dimensioned to exactly NRT by NZT. Each time the value of NRT or NZT is changed in program PCDSF, the statement dimensioning the array in program PCDSFPLT must be changed also.

3. The statements in this program which seem to do nothing but write variables to the screen actually have a more important function. Due to a bug in either the PRINTMATIC routines or in RM/FORTRAN, the PRINTMATIC routine DLINE, which is supposed to draw a straight line, refuses to work. It was discovered by accident that putting a WRITE statement near the call to the routine corrects the problem.

4. Program PCDSFPLT does not actually send anything to the printer; it merely creates a file whose name is given by the program variable NAME. If the value of NAME is 'filename.ext', then to print the file created by program PCDSFPLT, enter.

$$
\text { DPRINT filename.ext }
$$

DPRINT.EXE is a program supplied by PRINTMATIC. For this particular program the variable NAME is set to PCDSF.FIL, so to make a plot one would type:

DPRINT PCDSF.FIL 
Sample output

An example of the contour plot of the magnitude of the defect sensitivity factor is shown in Fig. 4.

COHL

AXIS

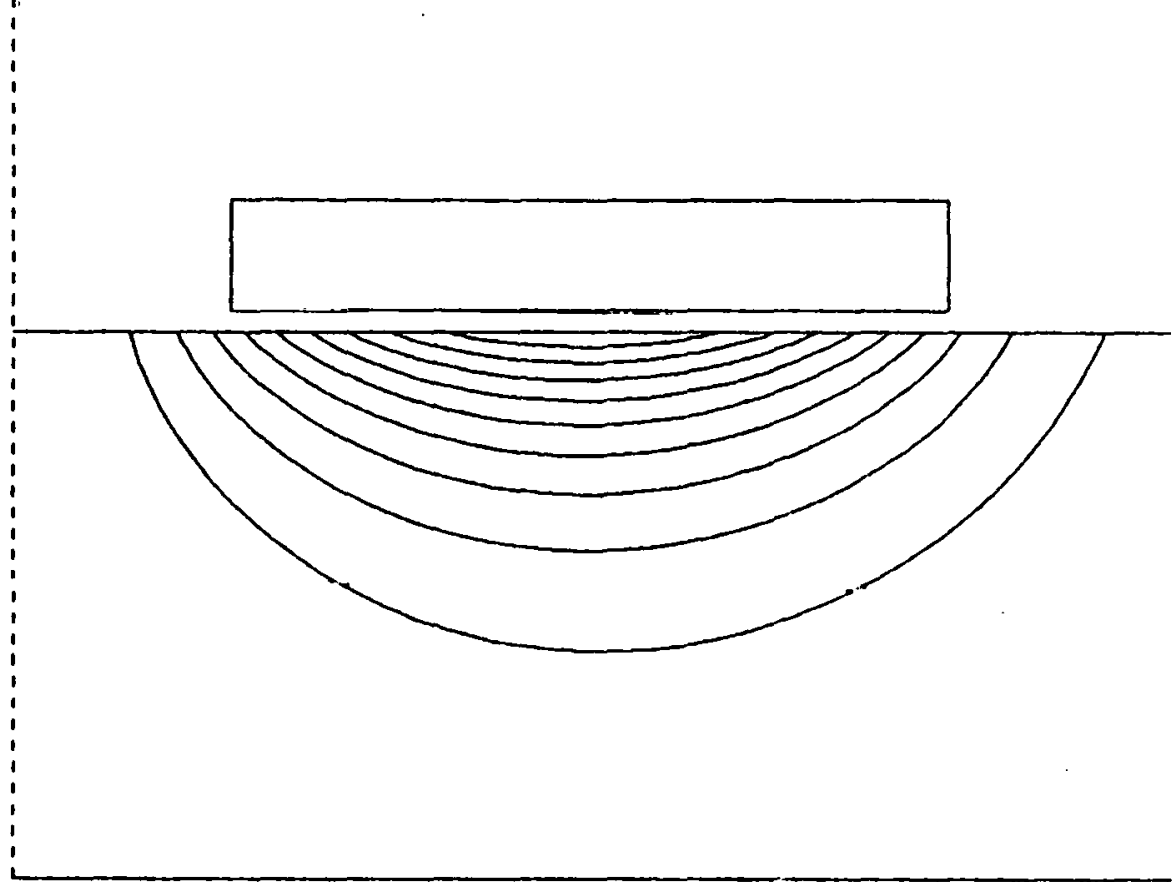

Fig. 4. Plot of the magnitude of the defect sensitivity factor. 


\section{Listing}

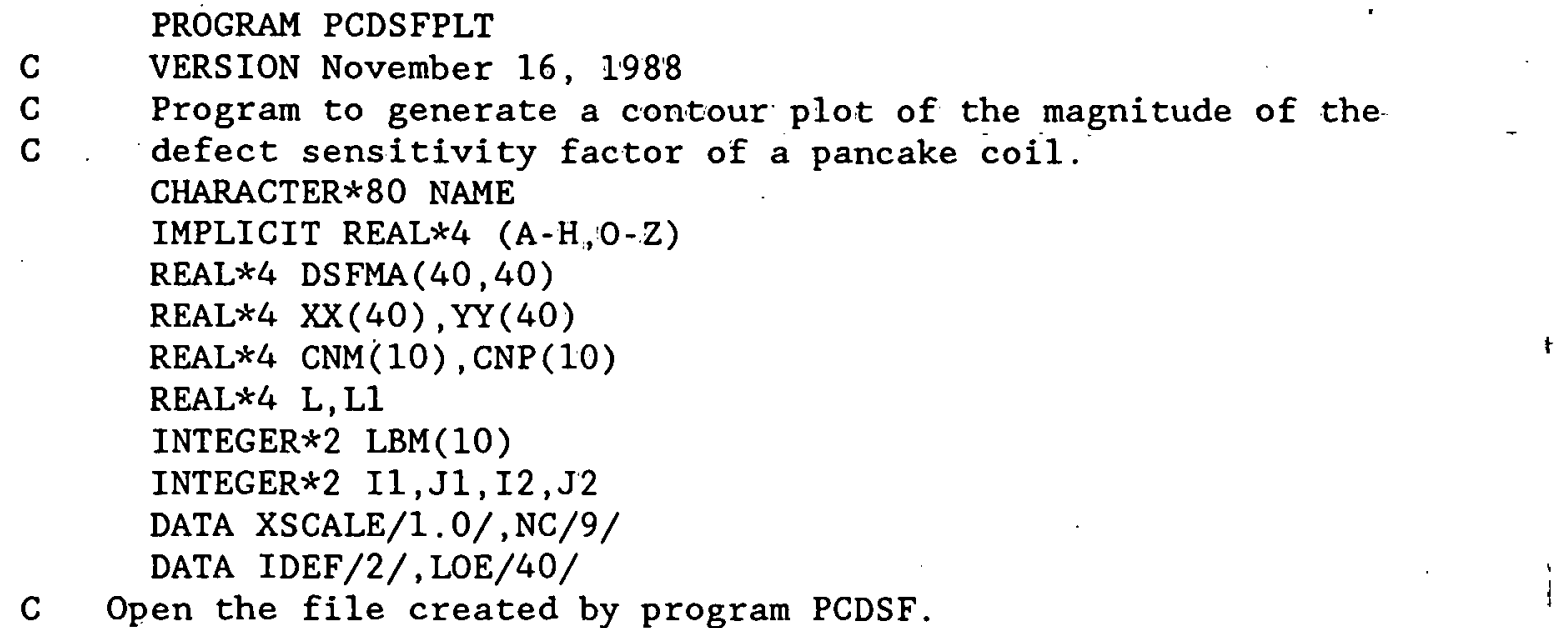

OPEN (LOE , FILE=' PCDSF. DAT' , STATUS=' OLD' )

$C$ Read in the coil information.

READ (LOE, *) NRT, NZT

READ (LOE, *) DELTAR, DELTAZ

$\operatorname{READ}(\mathrm{LOE}, *) \mathrm{R} 1, \mathrm{R} 2$

$\operatorname{READ}(\mathrm{LOE}, *) \mathrm{L}, \mathrm{LI}$

$\operatorname{READ}(\mathrm{LOE}, *) \mathrm{T} 1$

C Calculate the position of the data points in the

C normalized coordinate system.

DO $110 \mathrm{I}=0$, (NRT - 1 )

$\mathrm{XX}(\mathrm{I}+1)=\operatorname{REAL}(\mathrm{I}) *$ DELTAR

110 CONTINUE

DO $120 \quad I=0,(N Z T-1)$

$Y Y(I+1)=-((N Z T-I-1) *$ DELTAZ $)$

120 CONTINUE

C Set the label flags for the contours.

DO $130 \mathrm{I}=1,10$

$\operatorname{LBM}(I)=0$

130 CONTINUE

C Read in the data stored by program PCDSF.

$140 \operatorname{READ}(\mathrm{LOE}, *, \mathrm{END}=150$ ) NR, NZ, DSFM

c $140 \operatorname{READ}(\mathrm{LOE}, *, \mathrm{END}=150) \mathrm{NR}, \mathrm{NZ}, \mathrm{DUM}, \mathrm{DSFM}$

$\mathrm{NZ}=\mathrm{NZT}-\mathrm{NZ}+1$

IF (DSFM. GT . DSFMMAX) DSFMMAX $=$ DSFM

IF (DSFMMIN . EQ .0:) DSFMMIN=DSFM

IF (DSFM. LT . DSFMMIN) DSFMMIN $=$ DSFM

DSFMA $(N R, N Z)=D S F M$

GO TO 140

C Specify the values at which the contours are to be drawn.

150 VARMAG=DSFMMAX - DSFMMIN

CNTDIF=VARMAG $/(\mathrm{NC}+1)$

DO $160 \quad I=1, N C$

CNM $(I)=D S F M M A X-I *$ CNTDIF

160 CONTINUE 
C Call the necessary initialization routines.

NAME $=$ ' PCDSF, FIL'

CALL DINIT (NAME)

CALL DPLOT $(1 ., 1,6 ., 6,-0.1,1.9,-1 ., 1,0 ., 0$.

CALL DCTRDEF $(1,1,1,1,1)$

$C$ Draw the contours.

CALL DCNTOUR (XSCALE, XX, YY, DSFMA, CNM , LBM, NRT , NZT , NC, IDEF)

C Draw the plate.

write $(0, *) \mathrm{j} 2$

$\mathrm{X} 1=0$.

$\mathrm{Y} 1=0$.

CALL DRTOI $(\mathrm{X} 1, \mathrm{Y} 1, \mathrm{I} 1, \mathrm{~J} 1)$

$\mathrm{X} 2=2$.

$\mathrm{Y} 2=-\mathrm{T} 1$

CALL DRTOI (X2, Y2, I2, J2)

write $(0, *) \mathrm{j} 2$

CALL DLINE(I1, J1, I2, J1)

write $(0, *) \mathrm{j} 2$

CALL DLINE(I1, J 2, I 2, J2)

write $(0, *) \mathrm{j} 2$

$C$ Draw the coil.

$\mathrm{X} 1=\mathrm{R} 1$

$\mathrm{Y} 1=\mathrm{L} 1$

CALL DRTOI (X1, Y1 I I J 1$)$

$\mathrm{X} 2=\mathrm{R} 2$

$\mathrm{Y} 2=\mathrm{L}+\mathrm{L} 1$

CALL DRTOI (X2, Y2, I 2, J2)

writè $(0, *) \mathrm{J} 2$

CALL DLINE(I1,J1, I $2, \mathrm{~J} 1$ )

CALL DLINE(I1, J2, I 2, J 2)

CALL DLINE(I1,J1, I1, J 2)

$\operatorname{WRITE}(0, *) \mathrm{I} 2, \mathrm{~J} 1, \mathrm{I} 2, \mathrm{~J} 2$

CALL DLINE(I2,J1, I $2, \mathrm{~J} 2$ )

$\operatorname{WRITE}(0, *) \mathrm{I} 2, \mathrm{~J} 1, \mathrm{I} 2, \mathrm{~J} 2$

$C$ Draw the coil axis.

$\operatorname{WRITE}(n, *) I 1, \mathrm{~J} 1$

$\mathrm{X} 1=0$.

$Y 1=-T 1$

CALL DRTOI $(\mathrm{X} 1, \mathrm{Y} 1, \mathrm{I} 1, \mathrm{~J} 1)$

$\mathrm{X} 2=0$.

$\mathrm{Y} 2=0.6$

CALL DRTOI (X2, Y2, I $2, \mathrm{~J} 2)$

$\operatorname{WRITF}(0, *) \mathrm{I} 1, \mathrm{~J} 1, \mathrm{I} 2, \mathrm{~J} 2$

CALL DDASH(I1, J1, I2, J2, 1, 10, 10)

CALL DRTOT $(-0.1,0.7, \mathrm{I} 1, \mathrm{~J} 1)$

CALL DFONT (4, 'COIL', I1 , J1, 1 )

CALL DRTOI $(-0.1,0.62, \mathrm{I} 1, \mathrm{~J} 1)$

CALL DFONT (4, 'AXIS', I1, J1,1) 
CALL DFINIS.

write $(0, *) j 2$

stop

END 
PCFIX converts raw data to normalized impedance change

Program PCFIX converts raw pancake coil experimental data into the normalized impedance change in the pancake coil due to a defect. The raw data are read directly from voltmeters as the coil is moved over the surface of a plate by a program such as MIZSCN2. The voltmeters are connected to the vertical and horizontal channels of the Zetec MIZ-17. The MIZ-17 makes relative readings of the $x$ and $y$ components of the coil impedance. By comparing the measured change for a known amount of liftoff to the calculated change, both the phase (rotation) and gain of the signal are corrected. The values of the voltage readings with and without the lift-off must be measured and typed into the program.

\section{Summary}

1. Dimension arrays and declare variable types.

2. Initialize variables.

3. Calculate the constant by which the raw readings must be multiplied to convert them to the values of the normalized impedance change in the coil.

4. Read in a data point.

5. Subtract the reading taken on a part of a plate with no defects from the raw reading.

6. Multiply the reading by the constant obtained in 3 .

7. Loop to 4 until done.

8. Average the readings taken on opposite sides of the defect.

9. Write out the results.

\section{Variables}

CLOM ${ }^{\circ}$ The calculated value of the change in the magnitude of the normalized impedance due to the amount of additional lift-off we are using.

CLOP $\quad$ The calculated value of the change in the phase of the normalized impedance due to the amount of additional lift-off we are using.

LOD The number of the I/O unit connected to the file which is used for output.

LOE $\quad$ The number of the I/O unit connected to the file containing the raw data.

LOI The change in the imaginary part of the raw reading due to the additional lift-off.

LOR The change in the real part of the raw reading due to the additional lift-off.

RLOI $I^{\circ} \quad$ The imaginary part of the raw reading made with additional lift-off.

RLOR ${ }^{\circ}$ The real part of the raw reading made with additional lift-off.

SF The ratio of the magnitude of the calculated change in the normalized impedance to the change 
PD

in the raw reading due to additional lift-off.

The difference between the phase of the calculated change in the normalized impedance and the phase of the change in the raw reading due to additional lift-off.

ZEROI $\quad$ The imaginary part of the raw reading taken on a part of the plate with no defects.

ZEROR' The real part of the raw reading taken on a part of the plate with no defects.

ZLOI $\quad$ The imaginary part of the raw reading made on the same part of the plate where the readings for RLOR and RLOI were taken, but ZLOI is the reading without additional lift-off.

ZLOR $\quad$ The real part of the raw reading made on the same part of the plate where the readings for RLOR and RLOI were taken, but ZLOR is the reading without additional lift-off.

ZMAG

The magnitude of the change in the normalized impedance of the coil due to the defect.

ZPHA The phase of the change in the normalized impedance of the coil due to the defect.

\section{Notes}

1. The raw readings taken directly from the voltmeters by a program such as MIZSCN2 differ from the normalized impedance change in the coil by both an additive and a multiplicative constant. To determine the additive constant, we need only take readings on a part of the plate with no defects. This reading is subtracted from the raw readings. To determine the multiplicative constant, we find both the calculated and the experimental changes in readings due to a certain amount of additional lift-off. All subsequent raw readings are then multiplied by the ratio of the calculated value to the measured value. This directly normalizes the readings, and includes any amplification factors in the instrument.

2. This program averages the readings which precede the defect with those which follow the defect. The defect signal is supposed to be symmetric about the center of the defect. Any asymmetry should be due to random noise or to changes in the plate which we are not interested in. Averaging the signal will reduce the effect that such changes have on our results. 


\section{Listing}

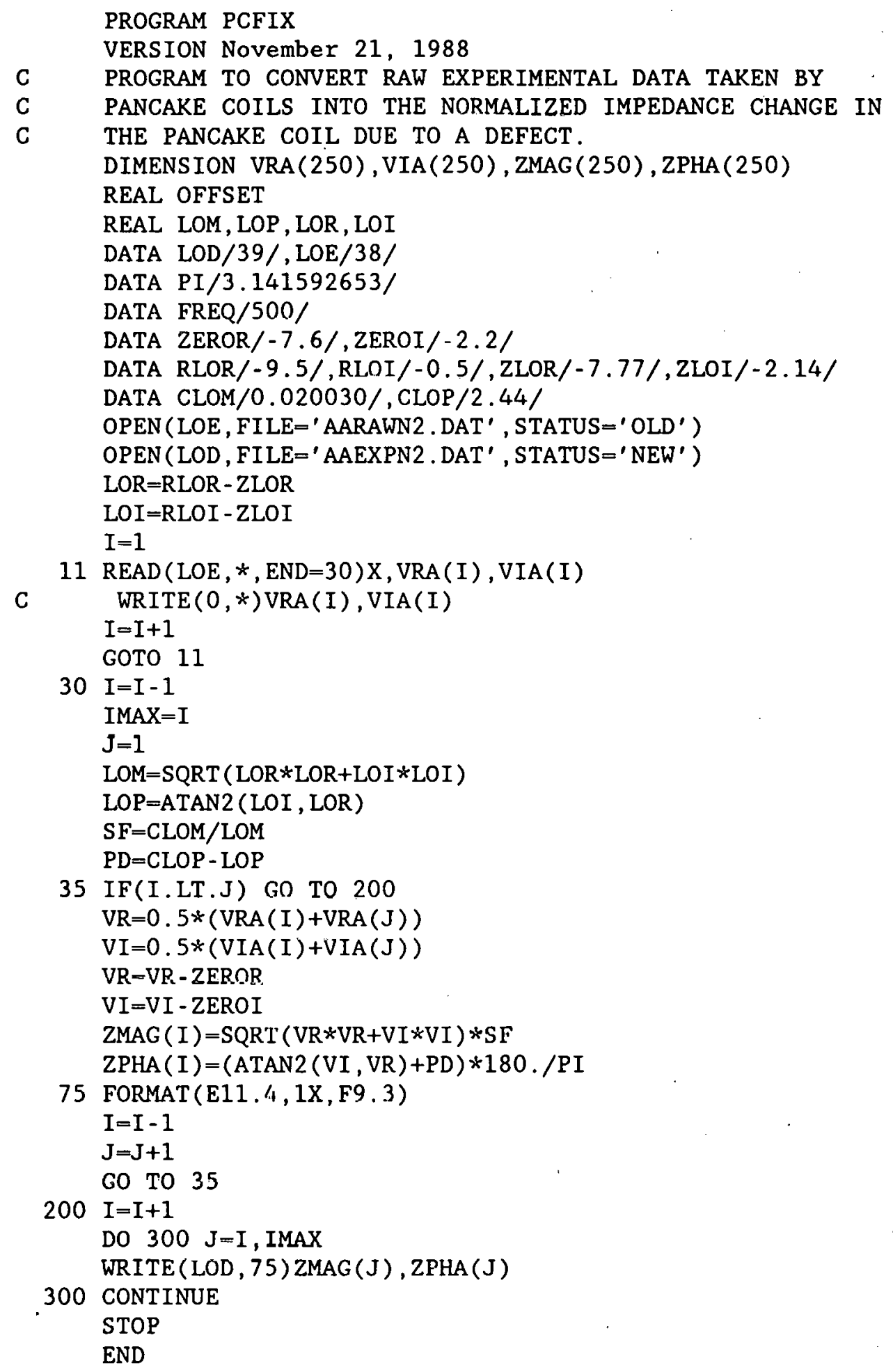


PCAVZSCN calculates defect impedance change, average over depth

Program PCAVZSCN calculates the change in the impedance of a pancake coil due to the presence of a defect in a conducting plate. It does the calculations for a number of different radial distances between the coil axis and the center of the defect, and it has the ability to divide the defect into smaller parts centered on the axis of the defect and to perform the calculations for each part separately, a method of treating the defect that usually gives more accurate results. The assumption is made that the defect has a constant cross section as the depth is varied, such as in a flat bottomed hole. If this is not the case, a weighted average with depth should be used. The output from PCAVZSCN is stored in the file PCAVZSCN.DAT.

\section{Summary}

1. Dimension arrays and declare variable types.

2. Initialize variables.

3. Begin the integration loops, calculating the expressions that are independent of the position of the defect.

4. Assign a value to FZD, the center of the part of the defect we wish to work with.

5. Do the calculations that depend only on the axial position of the defect.

6. Select a value for $\mathrm{RD}$, the radial distance between the coil and the part of the defect we are working with.

7. Complete the integration.

8. Loop to 6 until done.

9. Loop to 4 until done.

10. Write the results of the calculations to a file.

Variables

DELTAR $\quad$ The normalized radial distance between adjacent data points.

DFDEP $\quad$ The distance from the side of the plate where the defect is located to the bottom of the defect in inches.

DFDIAM" The diameter of the defect in inches.

DFM The magnitude of the change in the normalized impedance of the coil due to the defect.

DFP The phase of the change in the normalized impedance of the coil due to the defect.

DSFI The imaginary part of the defect sensitivity factor of the coil.

DSFR The real part of the defect sensitivity factor of the coil.

FREQ $\quad$ The operating frequency in hertz.

$\mathrm{L}^{\circ} \quad$ The length of the coil. The value is input in inches and normalized by the program.

L1. The lift-off of the coil. The value is input in 
inches and normalized by the program.

LOD $\quad$ The number of the I/O unit connected to the output data file.

LOU The number of the $I / O$ unit connected to the printer.

NRT* The number of different radial distances between the coil axis and the center of the defect at which the calculations are performed.

NS $\quad$ The side of the plate where the defect is located. If $\mathrm{NS}=1$, the defect is on the near side; if $\mathrm{NS}=2$, the defect is on the far side.

NZT $^{\circ} \quad$ The total number of parts into which the defect is divided along its axis when the calculations are performed.

Q6 The inductance of the coil in air.

$\mathrm{R}^{*}$ The inner radius of the coil. The value is input in inches and normalized by the program.

R2. The outer radius of the coil. The value is input in inches and normalized by the program.

R3 The mean radius of the coil in inches.

RD The normalized radial distance from the axis of the coil to the center of the defect.

$\mathrm{RHOI}^{\circ}$ The electrical resistivity of the plate in $\mu \Omega$ $\mathrm{cm}$.

T1. The thickness of the plate. The value is input in inches and normalized by the program.

$\mathrm{TRN}^{*} \quad$ The number of turns in the coil.

U1. The relative magnetic permeability of the plate.

VOLN The normalized volume of the defect.

WUSRR The product of the angular frequency, the relative magnetic permeability, the electrical conductivity, and the square of the mean coil radius.

2D The normalized distance from the side of the plate where the defect is located to the bottom of the defect. A negative number.

ZNDFI The illaginary part of tho ohange in the normalized impedance of the coil due to the defect.

ZNDFR The real part of the change in the normalized impedance of the coil due to the defect.

ZNIM The imaginary part of the normalized coil impedance with no defects present.

ZNRL The real part of the normalized coil impedance with no defects present.

\section{Notes}

1. Variable NZT controls the number of parts into which the defect is divided to perform the calculations. Since the theory upon which this program is based is more accurate for small defects, it is desirable to work with the defect in parts rather than as a whole. 
Integration Section of Program PCAVZSCN

Symbol definitions

The following are definitions of the symbols used to describe the program variables appearing in the integration section of this program. All lengths are normalized unless otherwise noted.

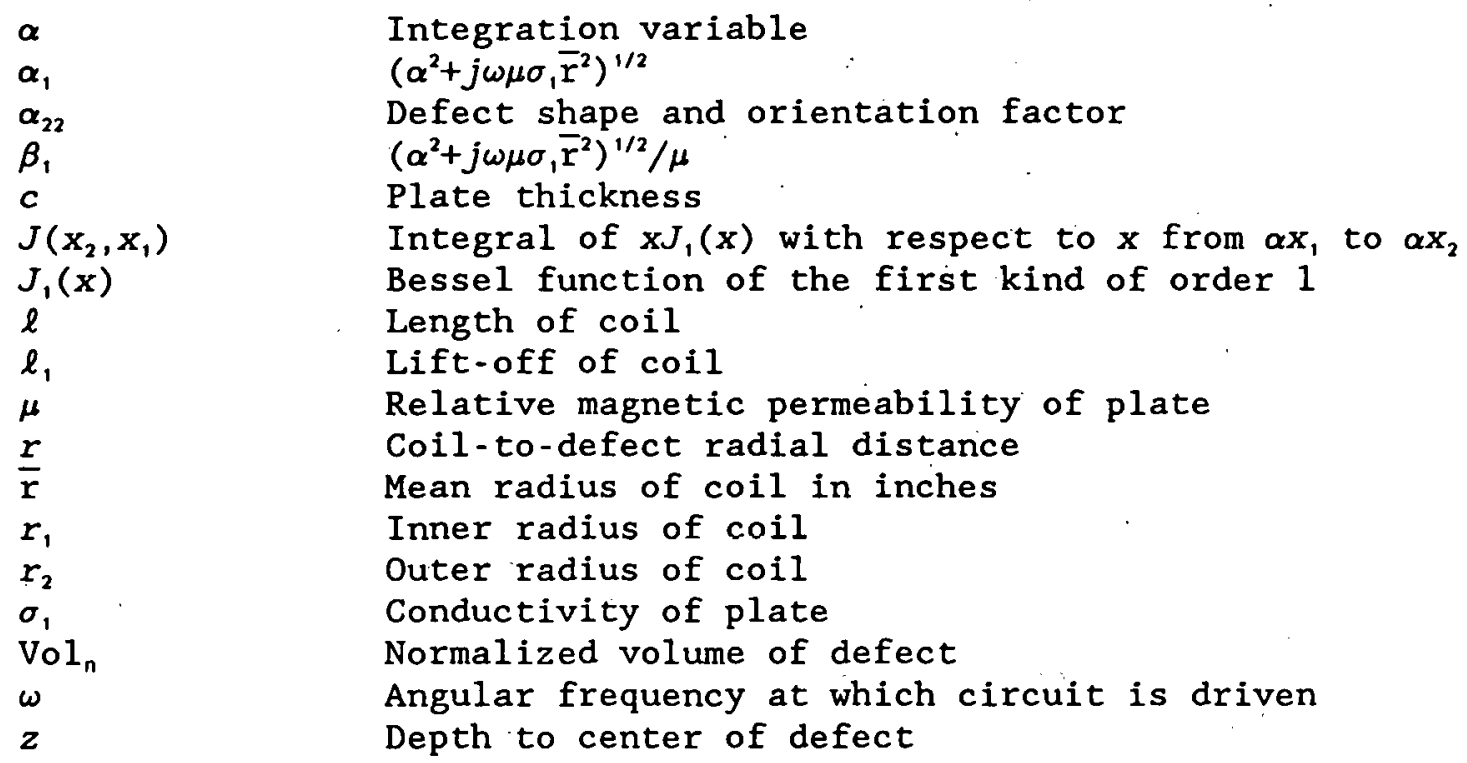

Variables appearing in the integration section

$\begin{array}{ll}\begin{array}{l}\text { Program } \\ \text { variable }\end{array} & \begin{array}{l}\text { Symbolic } \\ \text { equivalent } \\ \alpha \ell+\exp (-\alpha \ell)-1 \\ \text { A1 }\end{array} \\ \text { A2 } & \exp (-\alpha \ell) \\ \text { A2BI } & \operatorname{Im}\left[\alpha^{2}-\beta_{1}{ }^{2}\right] \\ \text { A2BR } & \operatorname{Re}\left[\alpha^{2}-\beta_{1}{ }^{2}\right] \\ \text { A3 } & \exp \left(-\alpha \ell_{1}\right) \\ \text { AMBI } & \operatorname{Im}\left[\left(\alpha-\beta_{1}\right)^{2}\right] \\ \text { AMBR } & \operatorname{Re}\left[\left(\alpha-\beta_{1}\right)^{2}\right] \\ \text { APBI } & \operatorname{Im}\left[\left(\alpha+\beta_{1}\right)^{2}\right] \\ \text { APBR } & \operatorname{Re}\left[\left(\alpha+\beta_{1}\right)^{2}\right] \\ \text { AVZDFI } & (\text { See note I3. } \\ \text { AVZDFR } & (\text { See note I3.) } \\ \text { B1 } & (\text { See note I2. } \\ \text { B2 } & \text { (See note I2.\} }\end{array}$




$$
\begin{aligned}
& \text { CFAC } \quad\left[J\left(r_{2}, r_{1}\right)\right]^{2} \\
& \text { CHECK (See note I2.) } \\
& \operatorname{CSDI} \quad \operatorname{Re}[\exp (\alpha, z)] \\
& \text { CSTI } \operatorname{Re}[\exp (-2 \alpha, c)] \\
& \text { CSZI } \quad \operatorname{Re}\left[\exp \left(-\alpha_{1}(2 c+z)\right)\right] \\
& \text { DENI } \operatorname{Im}\left[\left(\alpha+\beta_{1}\right)^{2}-\left(\alpha-\beta_{1}\right)^{2} \exp (-2 \alpha, c)\right] \\
& \text { DENR } \quad \operatorname{Re}\left[\left(\alpha+\beta_{1}\right)^{2}-\left(\alpha-\beta_{1}\right)^{2} \exp \left(-2 \alpha_{1} c\right)\right] \\
& \text { DFAC } \quad \frac{1}{\alpha^{3}}(1-\exp (-\alpha \ell)) \exp \left(-\alpha \ell_{1}\right) J\left(r_{2}, r_{1}\right) \mathrm{d} \alpha \\
& \text { ERR (See note I2.) } \\
& \text { FZD } z \text { (See note I4.\} } \\
& \text { ISTEPS \{See note I2.\} } \\
& \text { RI9 (See note I2.) } \\
& \text { RJ1 } J_{1}(\alpha r) \\
& \text { S1 do } \\
& \text { S2 (See note I2.) } \\
& \text { SMAIR } \quad \int_{0}^{\infty} \frac{2(\alpha \ell+\exp (-\alpha \ell)-1)\left[J\left(r_{2}, r_{1}\right)\right]^{2}}{\alpha^{6}} d \alpha \\
& \text { SMIMPI } \\
& \operatorname{Im}\left[\int_{0}^{\infty} \frac{(1-\exp (-\alpha l))^{2} \exp \left(-2 \alpha l_{1}\right)\left[J\left(r_{2}, r_{1}\right)\right]^{2}}{\alpha^{6}}\right. \\
& \left.\left[\frac{\left(\alpha^{2}-\beta_{1}{ }^{2}\right)-\left(\alpha^{2}-\beta_{1}{ }^{2}\right) \exp (-2 \alpha, c)}{\left(\alpha+\beta_{1}\right)^{2}-\left(\alpha-\beta_{1}\right)^{2} \exp (-2 \alpha, c)}\right] d \alpha\right] \\
& \left.\left[\frac{\left(\alpha^{2}-\beta_{1}{ }^{2}\right)-\left(\alpha^{2}-\beta_{1}{ }^{2}\right) \exp (-2 \alpha, c)}{\left(\alpha+\beta_{1}\right)^{2}-\left(\alpha-\beta_{1}\right)^{2} \exp (-2 \alpha, c)}\right] \mathrm{d} \alpha\right]
\end{aligned}
$$


SMZDFI

SMZDFR

$$
\begin{aligned}
\operatorname{Im} & {\left[\int_{0}^{\infty} \frac{1}{\alpha^{3}}(1-\exp (-\alpha l)) \exp \left(-\alpha l_{1}\right) J\left(r_{2}, r_{1}\right) J_{1}(\alpha r)\right.} \\
& \left.\alpha\left[\frac{\left(\alpha+\beta_{1}\right) \exp \left(\alpha_{1} z\right)-\left(\alpha-\beta_{1}\right) \exp \left(-\alpha_{1}(2 c+z)\right)}{\left(\alpha+\beta_{1}\right)^{2}-\left(\alpha-\beta_{1}\right)^{2} \exp \left(-2 \alpha_{1} c\right)}\right] \mathrm{d} \alpha\right]
\end{aligned}
$$

$\operatorname{Re}\left[\int_{0}^{\infty} \frac{1}{\alpha^{3}}(1-\exp (-\alpha \ell)) \exp \left(-\alpha \ell_{1}\right) J\left(r_{2}, r_{1}\right) J_{1}(\alpha r)\right.$

$$
\left.\alpha\left[\frac{\left(\alpha+\beta_{1}\right) \exp (\alpha, z)-\left(\alpha-\beta_{1}\right) \exp \left(-\alpha_{1}(2 c+z)\right)}{\left(\alpha+\beta_{1}\right)^{2}-\left(\alpha-\beta_{1}\right)^{2} \exp (-2 \alpha, c)}\right] \mathrm{d} \alpha\right]
$$

SNDI

SNTI

SNZI

TI

TR

TZI

TZR

$\mathrm{X}$

$\mathrm{X} 1$

$\mathrm{X} 1 \mathrm{X}$

$\mathrm{XJR} 1$

XJR2

$\mathrm{XL}$

XPDR

XPTR

XPZR

$X R D$

$\mathrm{XX}$

$\mathrm{XX1}$

$\mathrm{XY1}$

Y1

ZDI
$\operatorname{Im}[\exp (\alpha, z)]$

$-\operatorname{Im}[\exp (-2 \alpha, c)]$

$-\operatorname{Im}\left[\exp \left(-\alpha_{1}(2 c+z)\right)\right]$

$\operatorname{Im}[2 \alpha, c]$

$\operatorname{Re}[2 \alpha, c]$

$\operatorname{Im}\left[\alpha_{1}(2 c+z)\right]$

$\operatorname{Re}\left[\alpha_{1}(2 c+z)\right]$

$\alpha$

$\operatorname{Re}\left[\beta_{1}\right]$

$\operatorname{Re}[\alpha(\beta,-\alpha)]$

$J\left(r_{1}, 0\right)$

$J\left(r_{2}, 0\right)$

$\alpha \ell$

$\exp [\operatorname{Re}(\alpha ; z)]$

$\exp [\operatorname{Re}(-2 \alpha, c)]$

$\exp \left[\operatorname{Re}\left(-\alpha_{1}(2 c+z)\right)\right]$

$\alpha r$

$\alpha^{2}$

$\operatorname{Re}\left[\alpha\left(\alpha+\beta_{1}\right)\right]$

$\operatorname{Im}\left[\alpha\left(\alpha+\beta_{1}\right)\right]=\operatorname{Im}\left[\alpha\left(\beta_{1}-\alpha\right)\right]$

$\operatorname{Im}\left[\beta_{1}\right]$

$\operatorname{Im}[\alpha, z]$ 
ZDIM

$\operatorname{Im}\left[\frac{\left(\alpha+\beta_{1}\right) \exp \left(\alpha_{1} z\right)-\left(\alpha-\beta_{1}\right) \exp \left(-\alpha_{1}(2 c+z)\right)}{\left(\alpha+\beta_{1}\right)^{2}-\left(\alpha-\beta_{1}\right)^{2} \exp \left(-2 \alpha_{1} c\right)}\right]$

ZDR

$\operatorname{Re}[\alpha, z]$

ZDRL

$\operatorname{Re}\left[\alpha \frac{\left(\alpha+\beta_{1}\right) \exp (\alpha, z)-\left(\alpha-\beta_{1}\right) \exp (-\alpha,(2 c+z))}{\left(\alpha+\beta_{1}\right)^{2}-\left(\alpha-\beta_{1}\right)^{2} \exp \left(-2 \alpha_{1} c\right)}\right]$

ZIM

$\operatorname{Im}\left[\frac{\left(\alpha^{2}-\beta_{1}{ }^{2}\right)-\left(\alpha^{2}-\beta_{1}{ }^{2}\right) \exp (-2 \alpha, c)}{\left(\alpha+\beta_{1}\right)^{2}-\left(\alpha-\beta_{1}\right)^{2} \exp \left(-2 \alpha_{1} c\right)}\right]$

ZNDI

$\operatorname{Im}\left[\alpha\left(\alpha+\beta_{1}\right) \exp (\alpha, z)\right]$

ZNDR

$\operatorname{Re}\left[\alpha\left(\alpha+\beta_{1}\right) \exp (\alpha, z)\right]$

ZNUI

$\operatorname{Im}\left[\left(\alpha^{2}-\beta_{1}{ }^{2}\right)-\left(\alpha^{2}-\beta_{1}{ }^{2}\right) \exp (-2 \alpha, c)\right]$

ZNUR

$\operatorname{Re}\left[\left(\alpha^{2}-\beta_{1}{ }^{2}\right)-\left(\alpha^{2}-\beta_{1}{ }^{2}\right) \exp (-2 \alpha, c)\right]$

ZRL

$\operatorname{Re}\left[\frac{\left(\alpha^{2}-\beta_{1}{ }^{2}\right)-\left(\alpha^{2}-\beta_{1}{ }^{2}\right) \exp (-2 \alpha, c)}{\left(\alpha+\beta_{1}\right)^{2}-\left(\alpha-\beta_{1}\right)^{2} \exp (-2 \alpha, c)}\right]$

\section{Notes for the integration section}

I1. A number of the variables in the integration section are not always assigned their exact values but are approximated in certain cases to save time. For example, rather than calculate the exponential of a very small number that has its argument subtracted from it, the Maclaurin series expansion is sometimes used. Also, the exponential of a very large negative number is usually treated as zero.

I2. Several variables appear in the integration section of the program which play no part in the calculations being done. They are merely there to do such things as to determine the maximum step size which can be used while still accurately calculating the integrals.

13. Variables AVZDFR and AVZDFI are the averages of the elements in arrays SMZDFR and SMZDFI, respectively. For improved accuracy, the defect is divided into NZT parts to perform the calculations. The NZT elements of each array contain the calculations for these NZT parts. These elements are averaged to give the total effect of all of these parts on the impedances of the coils.

I4. The variable FZD is the normalized depth from the near side of the plate to the center of the part of the defect with which the program is working. It is not the depth of the center of the actual defect. 
Sample output

Printer output of program PCAVZSCN:

PCAVZSCN 20 POINTS USED FAR SIDE DEFECT TIME 8: 9:55 DATE 8/9/89 IN RAD OT RAD. LENGTH LIFTOFF CLADTH DF DEP

$\begin{array}{lllllll}\text { ACT } & 0.1000 & 0.4100 & 0.1000 & 0.0100 & 0.2500 & -0.0780\end{array}$

$\begin{array}{lllllll}\text { NOR } & 0.3922 & 1.6078 & 0.3922 & 0.0392 & 0.9804 & -0.3059\end{array}$

RBAR $0.2550 \quad F R E Q=5.000000 E+02$ RHO $=4.0540$ PERM $=1.000$ WUSRR $=4.0853$ NORM IMPD:RL 0.155825 IM 0.832098 AIR IND 6.252919E-03 VOLN 2.1905E-02

Partial listing of file PCAVZSCN.DAT:

$0.1550 \mathrm{D}-06 \quad 54.632$

$0.1390 D-05 \quad 54.602$

$0.3832 \mathrm{D}-05 \quad 54.540$

$0.7428 \mathrm{D}-05 \quad 54.448$

$0.1210 \mathrm{D}-04 \quad 54.322$

$0.1774 \mathrm{D}-04 \quad 54.162$

$0.2423 D-04 \quad 53.966$

$0.3143 \mathrm{D}-04 \quad 53.733$

$0.3919 \mathrm{D}-04 \quad 53.459$

$0.4733 \mathrm{D}-04 \quad 53.144$ 


\section{Listing}

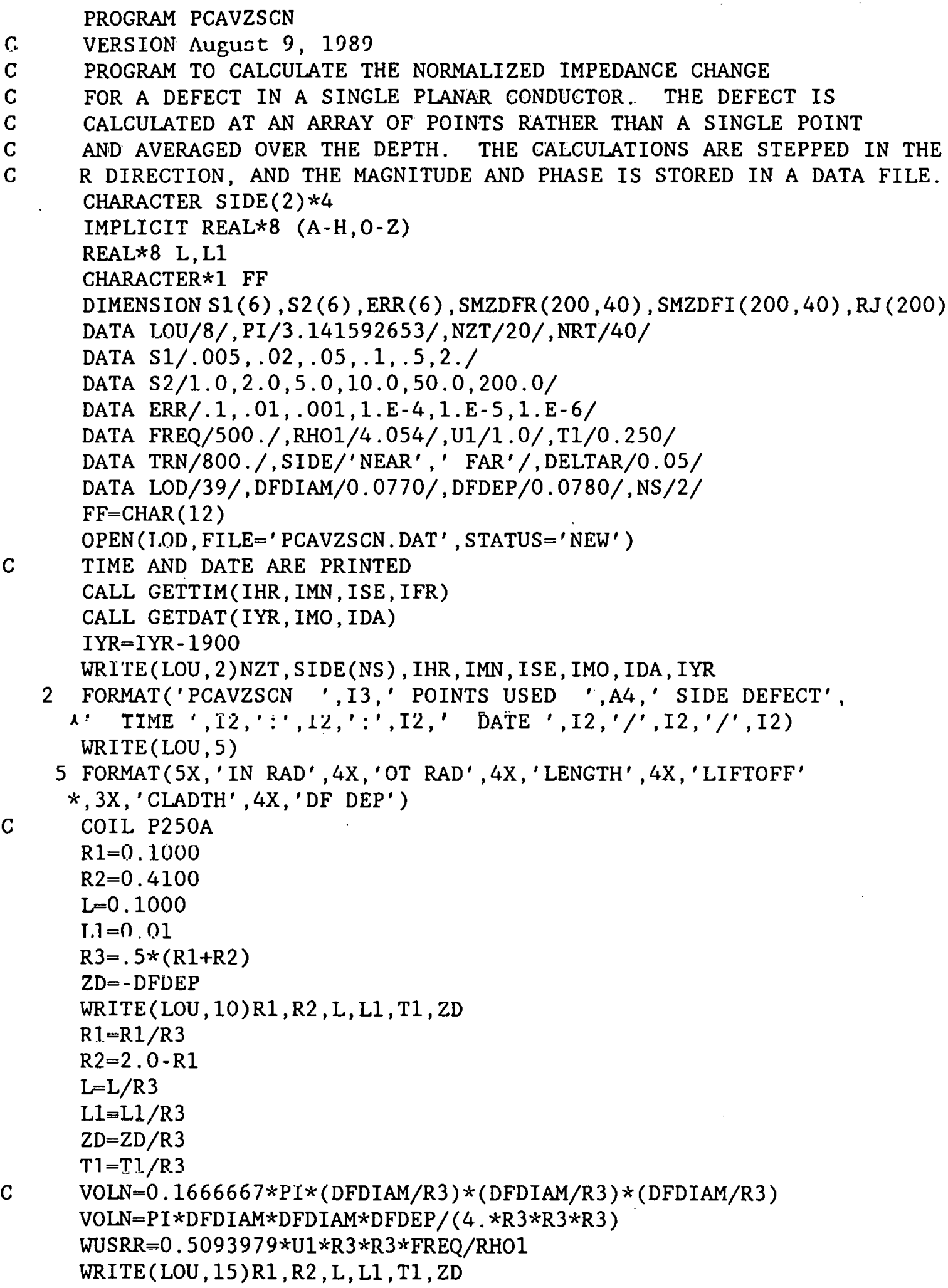




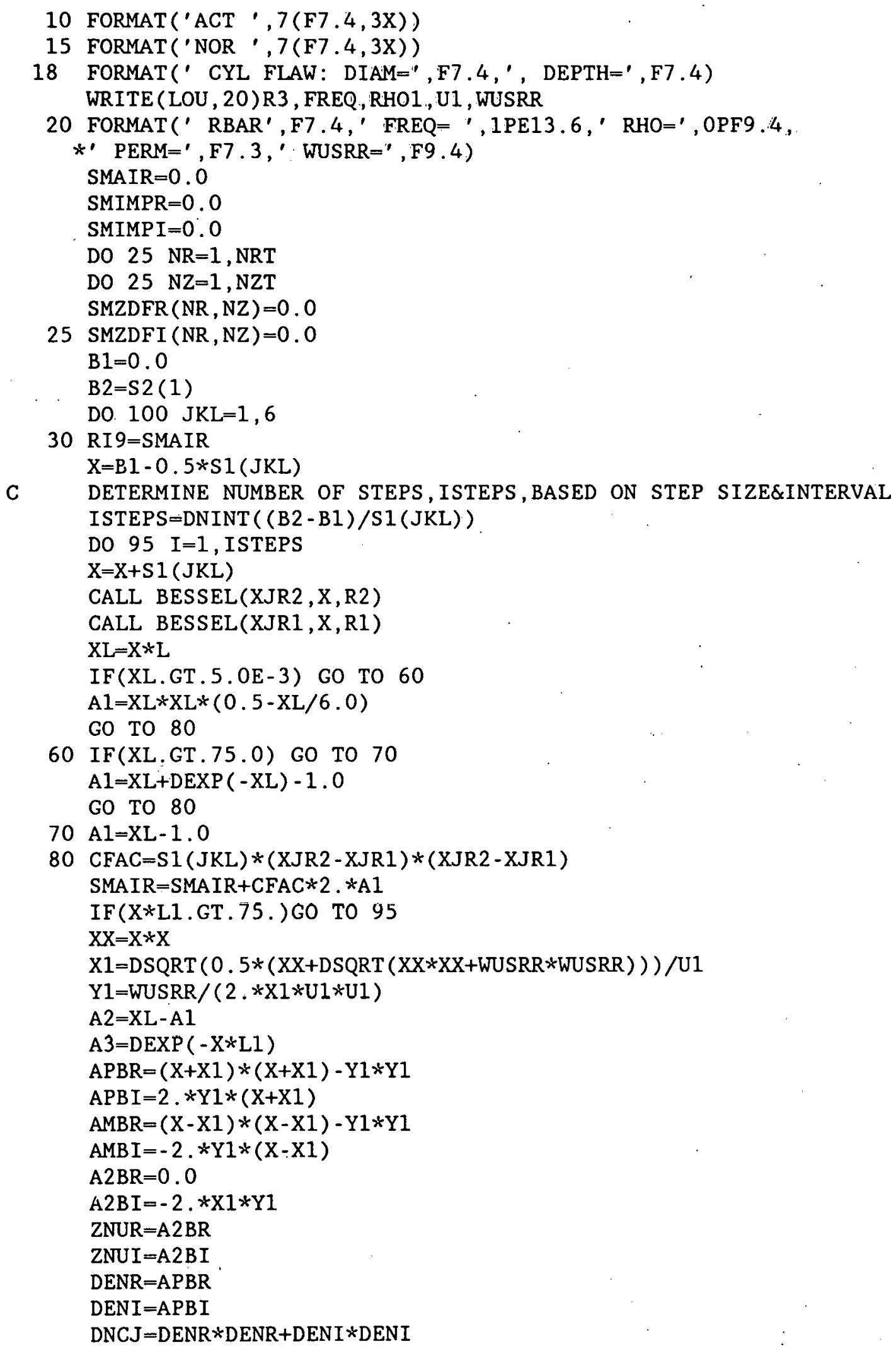




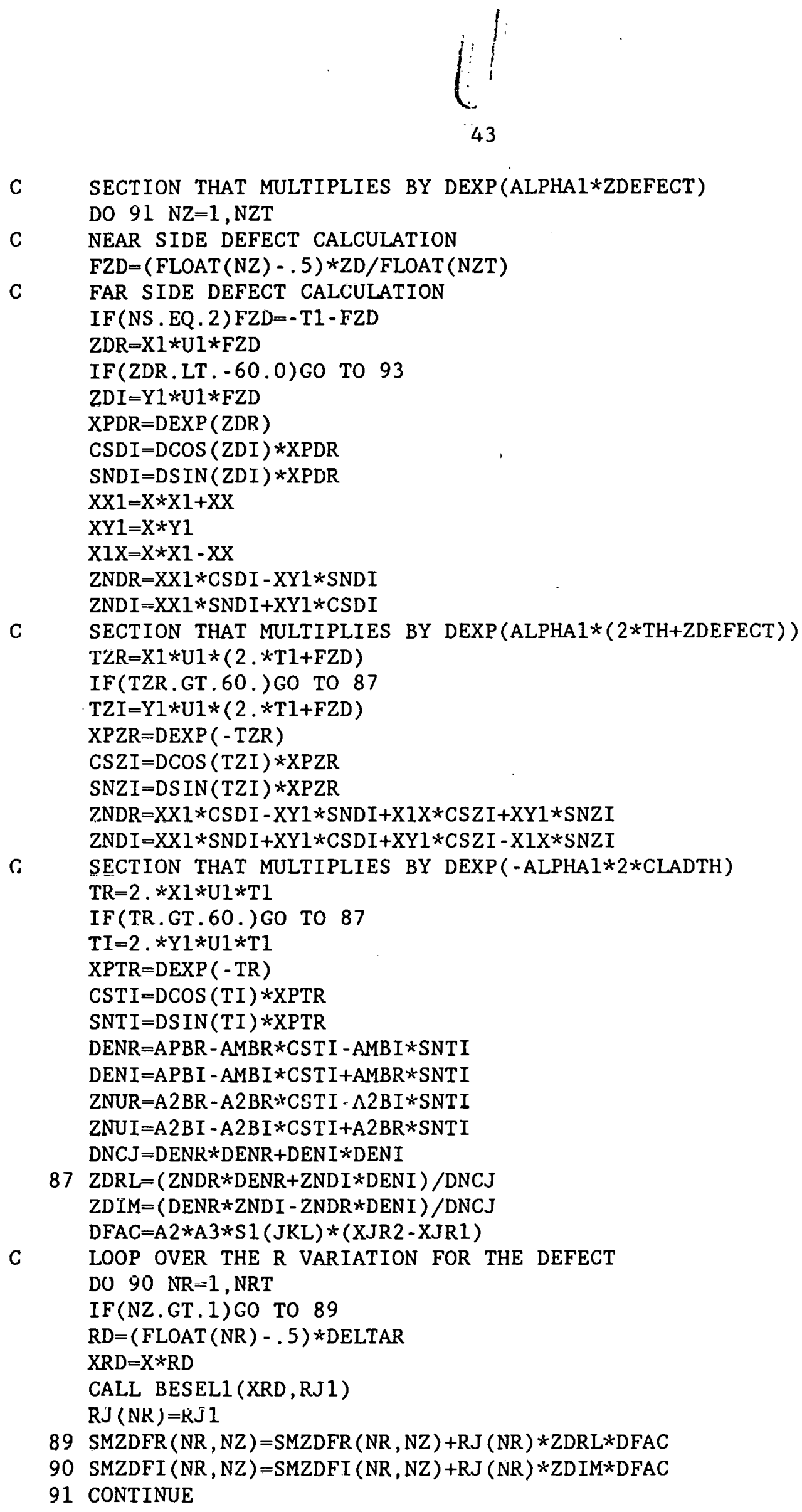


$93 \mathrm{ZRL}=(\mathrm{ZNUR} * \mathrm{DENR}+\mathrm{ZNUI} * \mathrm{DENI}) / \mathrm{DNCJ}$

$\mathrm{ZIM}=(\mathrm{DENR} * \mathrm{ZNUI}-\mathrm{ZNUR} * \mathrm{DENI}) / \mathrm{DNCJ}$

SMIMPR $=S M I M P R+A 2 * A 2 * A 3 * A 3 * Z R L * C F A C$

SMIMPI $=S M I M P I+A 2 * A 2 * A 3 * A 3 * Z I M * C F A C$

95 CONTINUE

$\mathrm{B} 1=\mathrm{B} 2$

$\mathrm{B} 2=\mathrm{B} 2+\mathrm{S} 2(\mathrm{JKL})$

CHECK $=($ SMAIR - RI9) $/$ SMAIR

IF (ABS (CHECK) .GT.ERR (JKL)) GO TO 30

100 CONTINUE

$135 \mathrm{Q} 6=0.0254 * 4.0 \mathrm{E}-7 * \mathrm{TRN} * \mathrm{TRN} * \mathrm{R} 3 * \mathrm{PI} * \mathrm{PI} * \mathrm{SMAIR} /(\mathrm{L} *(\mathrm{R} 2-\mathrm{R} 1)) * * 2$

ZNIM $=($ SMIMPR+SMAIR $) /$ SMAIR

ZNRL $=-$ SMIMPI/SMAIR

WRITE (LOU, 140) ZNRL, ZNIM, Q6, VOLN

140 FORMAT(' NORM IMPD:RL',F10.6,' IM', F10.6,

*'AIR IND', 1PE13.6,' VOLN',1PE11.4)

150 FORMAT(' NORM CHG:RL ',OPF10.6,' IM', OPF10.6,

*' MAG ',OPF10.6,' PHA ',OPF7.2)

160 FORMAT(' NORM DSF:RL',1PE11.4,' IM',1PE11.4,' VOLN',1PE11.4)

DO $200 \mathrm{NR}=1$, NRT

$\mathrm{RD}=($ FLOAT $(\mathrm{NR})-.5) * \mathrm{DELTAR}$

C AVERAGE OVER DEFECT POINTS AT DIFFERENT DEPTHS

AVZDFR $=0.0$

AVZDFI $=0.0$

DO $220 \mathrm{NZ}=1$, NZT

AVZDFR=AVZDFR+SMZDFR $(N R, N Z)$

220 AVZDFI=AVZDFI+SMZDFI (NR, NZ)

$A V Z D F R=A V Z D F R / F L O A T(N Z T)$

AVZDFI $=$ AVZDFI /FLOAT (NZT)

$\mathrm{DSFR}=-1.5 *$ WUSRR $*(\mathrm{AVZDFR} * \mathrm{AVZDFR}-\mathrm{AVZDFI} * \mathrm{AVZDFI}) /(\mathrm{SMAIR} * \mathrm{PI})$

$D S F I=-1.5 *$ WUSRR $* 2.0 * A V Z D F R * A V Z D F I /(S M A I R * P I)$

$\mathrm{ZNDFR}=\mathrm{VOLN} * \mathrm{DSFR}$

$\mathrm{ZNDFI}=\mathrm{VOLN} * \mathrm{DSFI}$

$D F M=V O L N * S Q R T(D S F R * D S F R+D S F I * D S F I)$

$\mathrm{DFP}=\mathrm{ATAN} 2(\mathrm{DSFI}, \mathrm{DSFR}) * 180 . / \mathrm{PI}$

200 WRITE (LOD , 178) DFM, DFP

178 FORMAT (D $1.1 .4,1 \mathrm{X}, \mathrm{F} 8.3$ )

1001 STOP

END 
PCAVVSCN calculates defect impedance change, average over volume

Program PCAVVSCN calculates the change in the impedance of a pancake coil due to the presence of a flat-bottomed hole in a conducting plate. It first calculates the defect sensitivity factor for a lattice of points that extends from the coil axis to the outer edge of the coil field, as shown in Fig. 3. Although this method of averaging over the dimensions of the defect is not mathematically correct, it usually gives more accurate results than assuming that the defect is a point or only averaging over the defect depth. Once the defect sensitivity factor has been computed, the defect can be considered to be at any radial location with respect to the coil and its average effect calculated by summing over the defect sensitivity factor values at the different radial positions multiplied by the volume of that particular element. In Fig. 5 we show the relationship of a flat-bottomed hole to the coil center, looking down on the plate.

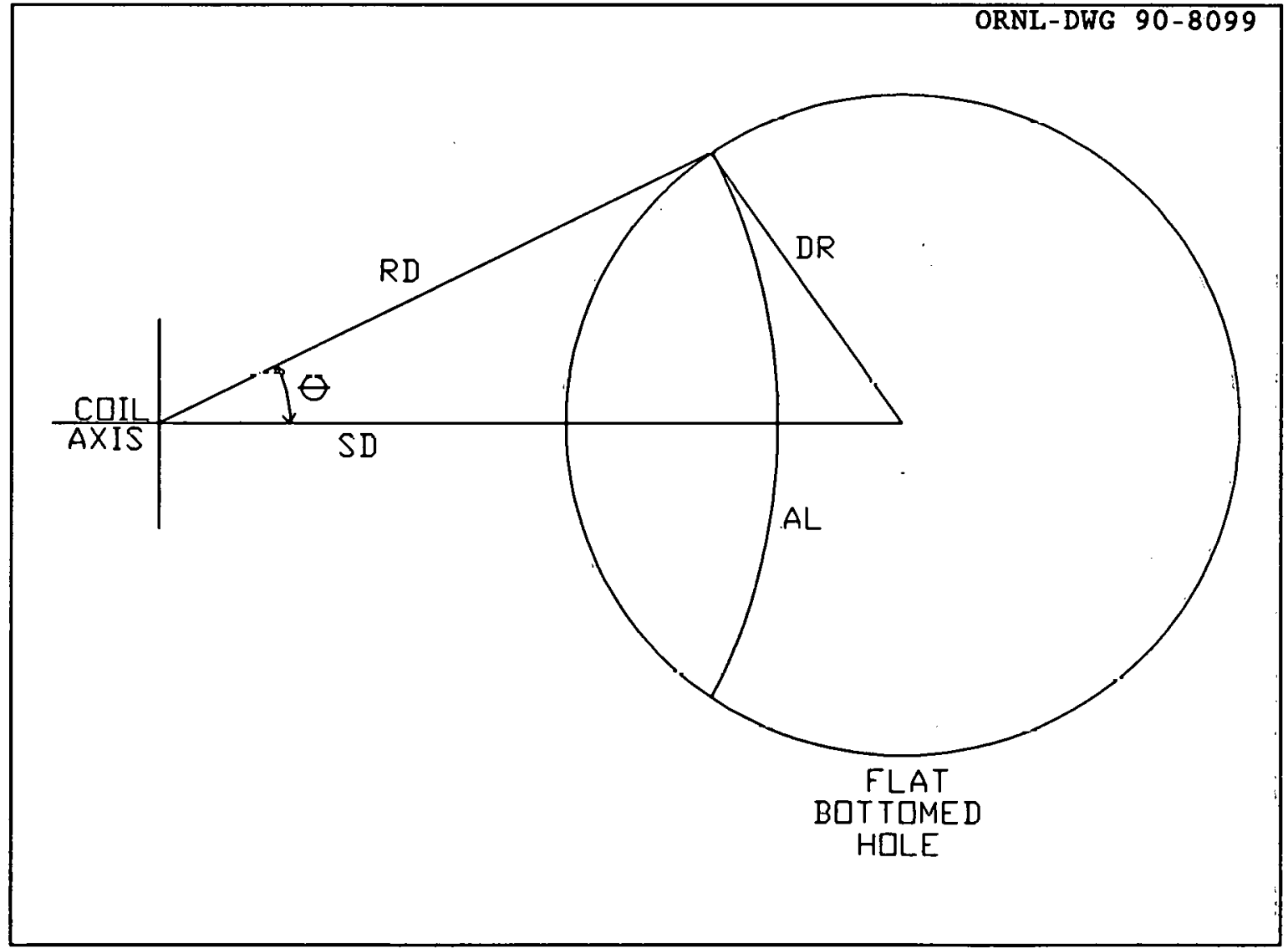

Fig. 5. Top view of flat-bottomed hole referenced to the coil axis.

The defect signal is first averaged over the defect depth in a manner similar to the PCAVZSCN program. Then the arc length AL is multiplied by the real and imaginary value of the average defect signal and this is summed as the distance from the coil axis RD is stepped from one side of 
the defect to the other. This is repeated for different values of the distance from the coil axis to the defect center, SD. This simulates the coil scanning across the defect. It should be noted that the defect sensitivity factor integration does not have to be repeated as SD increases or if another size defect is used if the lattice of points is fine enough. However, rather than choose the lattice fine enough to cover all defect depths presently stored in PCAVVSCN, we have set the program up so that it will compute a new lattice for each defect depth.

The arc length is calculated by the expression:

$$
\mathrm{AL}=2 \mathrm{RD}\left[\cos ^{-1}\left(\frac{\mathrm{SD}^{2}+\mathrm{RD}^{2}-\mathrm{DR}^{2}}{2 \mathrm{RD} \mathrm{SD}}\right)\right]
$$

unless the defect encloses the coil origin. In this case, the arc length is :

$$
\mathrm{AL}=2 \pi \mathrm{RD}
$$

as long as RD is less than DR - SD. Then the expression in Eq. (18) should be used.

The defect volume can also be calculated by multiplying the arc length AL by the incremental step in RD. A comparison of the volume calculated by this method shows an agreement to within 0.18 if the lattice is fine enough to have 40 points across the defect. A lattice of 20 points will give an error of $0.3 \%$.

The defect signal is averaged over the defect volume using the expression:

$$
A V Z D R+j A V Z D I=\sum_{N R} \frac{A L(A V Z D F R(N R)+j A V Z D F I(N R))}{\sum_{N R} A L}
$$

The division by the summation of the arc length in Eq. (19) helps reduce any errors in the computation of the defect volume and furnishes the proper normalization. The limit of the summation is only done for NR values that lie within the defect.

The output for the program is written on the file FORT9.

\section{Summary}

1. Dimension arrays and declare variable types.

2. Initialize variables.

3. Begin the integration loops, calculating the expressions that are independent of the position of the defect.

4. Assign a value to FZD, the center of the part of the defect we wish to work with.

5. Do the calculations that depend only upon the axial position of the defect.

6. Select a value for $\mathrm{RD}$, the radial distance between the coil and the part of the defect we are working with. 
7. Complete the integration.

8. Loop to 6 until done.

9. Loop to 4 until done.

10. Write the results of the calculations to a file.

Variables

ANG

The angle of half the arc length $\mathrm{AL}, \theta$ in Figure 5.

DELTAR' The normalized radial distance between adjacent data points.

DFDEP $\quad$ The distance from the side of the plate where the defect is located to the bottom of the defect in inches.

DFDIAM* The diameter of the defect in inches.

DFM The magnitude of the change in the normalized impedance of the coil due to the defect.

DFP The phase of the change in the normalized impedance of the coil due to the defect.

DR The normalized defect radius.

DRR The normalized defect radius best described by the NDT points.

DSFI The imaginary part of the defect sensitivity factor of the coil.

DSFR The real part of the defect sensitivity factor of the coil.

FREQ* The operating frequency in hertz.

$\mathrm{L}^{\circ}$

The length of the coil. The value is input in inches and normalized by the program.

L1 The lift-off of the coil. The value is input in inches and normalized by the program.

LOD $\quad$ The number of the I/O unit connected to the output data file.

LOU The number of the $I / O$ unit connected to the printer.

NDF The number denoting the particular defect in the array of defects.

NDT The number of radial points across the defect.

NRD The integer that denotes the radial location at which the defect sensitivity is calculated.

NRT. The total number of radial points at which the defect sensitivity calculations are performed.

NRTT The total number of points in the radial direction at which the averaged impedance change is calculated. Presently set to $2 / 3$ of NRT.

NS $\quad$ The side of the plate where the defect is located. If $N S=1$, the defect is on the near side; if $N S=2$, the defect is on the far side.

NZT ${ }^{*} \quad$ The total number of parts into which the defect. is divided along its axis when the calculations: are performed. 
Q6 The inductance of the coil in air.

$\mathrm{R}^{\circ}$ The inner radius of the coil. The value is: input

R2. The outer radius of the coil. The value is input.

R3 The mean radius of the coil in inches.

RD The normalized radial distance from the axis of the coil to the center of the defect.

RHO1 $1^{\circ}$ The electrical resistivity of the plate in $\mu \Omega$ $\mathrm{cm}$.

T1 The thickness of the plate. The value is input in inches and normalized by the program.

TRN $\quad$ The number of turns in the coil.

U1 The relative magnetic permeability of the plate.

VOLN The normalized volume of the defect.

WIISRR The product of the angular frequency, the relative magnetic permeability, the electrical conductivity, and the square of the mean coil radius.

2D The normalized distance from the side of the plate where the defect is located to the bottom of the defect. A negative number.

ZNDFI The imaginary part of the change in the normalized impedance of the coil due to the defect.

ZNDFR The real part of the change in the normalized impedance of the coil due to the defect.

ZNIM The imaginary part of the normalized coil impedance with no defects present.

ZNRL The real part of the normalized coil impedance with no defects present.

Notes

1. Variable NZT controls the number of parts into which the defect is divided to perform the calculations. Since the theory upon which this program is based is more accurate for small defects, it is desirable to work with the defect in parts rather than as a whole. 
Integration Section of Program PCAVVSCN

\section{Symbol definitions}

The following are definitions of the symbols used to describe the program variables appearing in the integration section of this program. All lengths are normalized unless otherwise noted.

\begin{tabular}{|c|c|}
\hline$\alpha$ & Integration variable \\
\hline$\alpha_{1}$ & \\
\hline$\alpha_{22}$ & Defect shape and orientation factor \\
\hline $\begin{array}{l}\beta_{1} \\
c\end{array}$ & $\begin{array}{l}\left(\alpha^{2}+j \omega \mu \sigma, r^{2}\right)^{1 / 2} / \mu \\
\text { Plate thickness }\end{array}$ \\
\hline $\begin{array}{l}J\left(x_{2}, x_{1}\right) \\
J,(x) \\
\ell\end{array}$ & $\begin{array}{l}\text { Integral of } x J_{1}(x) \text { with respect to } x \text { from } \alpha x_{1} \text { to } \alpha x_{2} \\
\text { Bessel function of the first kind of order } 1 \\
\text { Length of coil }\end{array}$ \\
\hline$\ell_{1}$ & Lift-off of coil \\
\hline$\mu$ & Relative magnetic permeability of plate \\
\hline$\underline{r}$ & Coil-to-defect radial distance \\
\hline$\overline{\mathbf{r}}$ & Mean radius of coil in inches \\
\hline$r_{1}$ & Inner radius of coil \\
\hline$r_{2}$ & Outer radius of coil \\
\hline$\sigma_{1}$ & Conductivity of plate \\
\hline $\begin{array}{l}V_{0} I_{n} \\
\omega\end{array}$ & Normalized volume of defect \\
\hline $\begin{array}{l}\omega \\
z\end{array}$ & Angular frequency at which ci \\
\hline & Depth to center of defect \\
\hline
\end{tabular}

Variables appearing in the integration section

\begin{tabular}{|c|c|}
\hline $\begin{array}{l}\text { Program } \\
\text { variable } \\
\text { A1 }\end{array}$ & $\begin{array}{l}\text { Symbolic } \\
\text { equivalent } \\
\alpha \ell+\exp (-\alpha \ell)-1\end{array}$ \\
\hline A2 & $1-\exp (-\alpha \ell)$ \\
\hline $\mathrm{A} 2 \mathrm{BI}$ & $\operatorname{Im}\left[\alpha^{2}-\beta_{1}{ }^{2}\right]$ \\
\hline $\mathrm{A} 2 \mathrm{BR}$ & $\operatorname{Re}\left[\alpha^{2}-\beta_{1}{ }^{2}\right]$ \\
\hline A3 & $\exp \left(-\alpha \ell_{1}\right)$ \\
\hline AMBI & $\operatorname{Im}\left[\left(\alpha-\beta_{1}\right)^{2}\right]$ \\
\hline AMBR & $\operatorname{Re}\left[\left(\alpha-\beta_{1}\right)^{2}\right]$ \\
\hline APBI & $\operatorname{Im}\left[\left(\alpha+\beta_{1}\right)^{2}\right]$ \\
\hline APRR & $\operatorname{Re}\left[\left(\alpha+\beta_{1}\right)^{2}\right]$ \\
\hline AVZDFI (NR) & \{See note I3.\} \\
\hline AVZDFR(NR) & (See note I3.) \\
\hline B1 & $\{$ See note 12.$\}$ \\
\hline B2 & (See note 12. ) \\
\hline
\end{tabular}




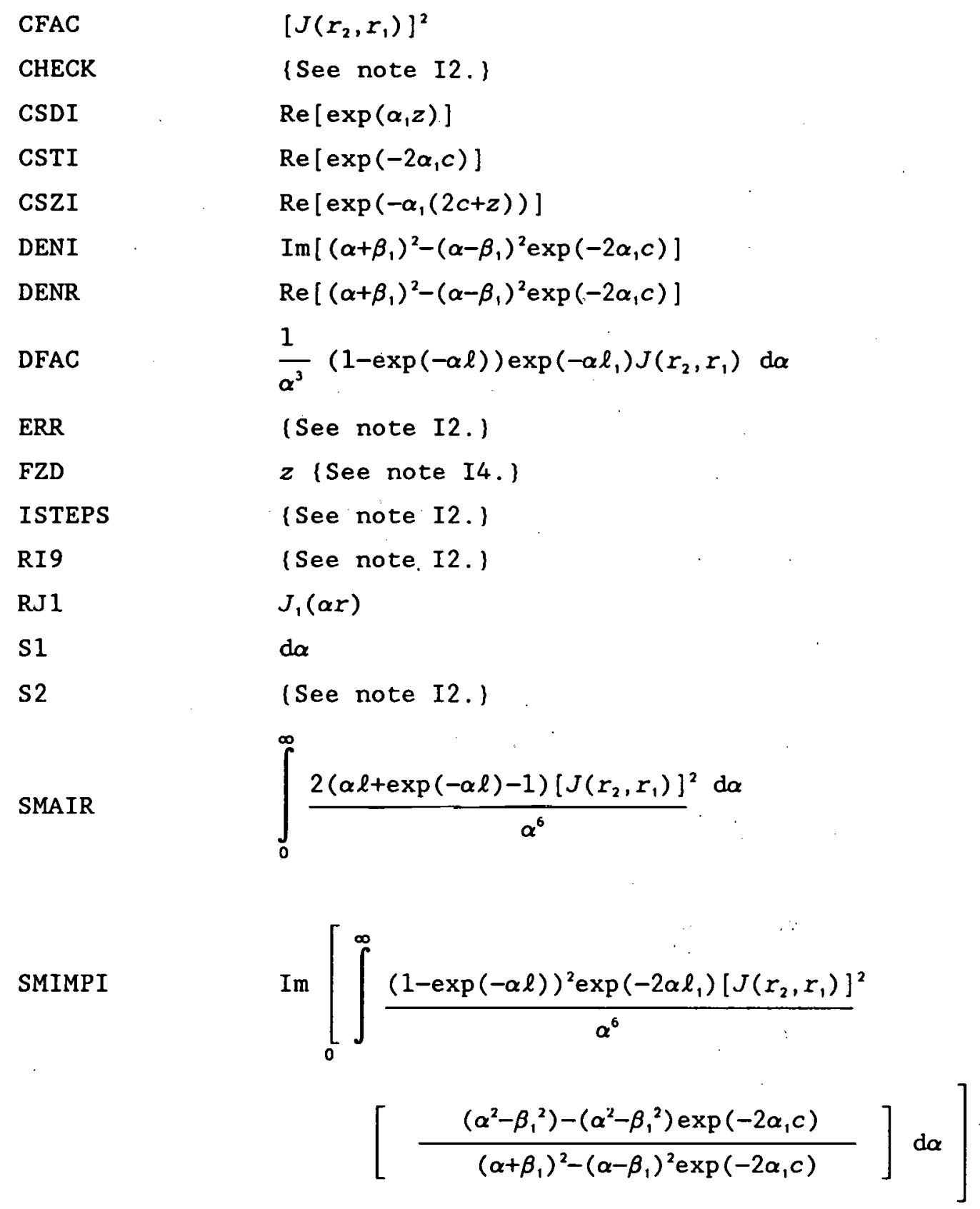


SMIMPR

$\operatorname{Rc} \int_{0}^{\infty} \frac{(1-\exp (-\alpha, l))^{2} \exp \left(-2 \alpha \ell_{1}\right)\left[J\left(r_{2}, r_{1}\right)\right]^{2}}{\alpha^{6}}$

$$
\left.\left[\frac{\left(\alpha^{2}-\beta_{1}{ }^{2}\right)-\left(\alpha^{2}-\beta_{1}{ }^{2}\right) \exp \left(-2 \alpha_{1} c\right)}{\left(\alpha+\beta_{1}\right)^{2}-\left(\alpha-\beta_{1}\right)^{2} \exp (-2 \alpha, c)}\right] d \alpha\right]
$$

SMZDFI

$\operatorname{Im}\left[\int_{0}^{\infty} \frac{1}{\alpha^{3}}(1-\exp (-\alpha \ell)) \exp \left(-\alpha \ell_{1}\right) J\left(r_{2}, r_{1}\right) J_{1}(\alpha r)\right.$

$$
\left.\left[\frac{\left(\alpha+\beta_{1}\right) \exp (\alpha, z)-\left(\alpha-\beta_{1}\right) \exp (-\alpha,(2 c+z))}{\left(\alpha+\beta_{1}\right)^{2}-\left(\alpha-\beta_{1}\right)^{2} \exp (-2 \alpha, c)}\right] \mathrm{d} \alpha\right]
$$

SMZDFR

$$
\begin{aligned}
\operatorname{Re} & {\left[\int_{0}^{\infty} \frac{1}{\alpha^{3}}(1-\exp (-\alpha l)) \exp \left(-\alpha l_{1}\right) J\left(r_{2}, r_{1}\right) J_{1}(\alpha r)\right.} \\
& {\left.\left[\frac{\left(\alpha+\beta_{1}\right) \exp \left(\alpha_{1} z\right)-\left(\alpha-\beta_{1}\right) \exp \left(-\alpha_{1}(2 c+z)\right)}{\left(\alpha+\beta_{1}\right)^{2}-\left(\alpha-\beta_{1}\right)^{2} \exp (-2 \alpha, c)}\right] \mathrm{d} \alpha\right] }
\end{aligned}
$$

SNDI

SNTI

SNZI

TI

TR

TZI

TZR

$\mathrm{X}$

$\mathrm{X} 1$

$\mathrm{X} 1 \mathrm{X}$

XJR1

XJR2

$\mathrm{XI}$

XPDR
$\operatorname{Im}[\exp (\alpha, z)]$

$-\operatorname{Im}[\exp (-2 \alpha, c)]$

$-\operatorname{Im}\left[\exp \left(-\alpha_{1}(2 c+z)\right)\right]$

$\operatorname{Im}[2 \alpha, c]$

$\operatorname{Re}[2 \alpha, c]$

$\operatorname{Im}\left[\alpha_{1}(2 c+z)\right]$

$\operatorname{Re}\left[\alpha_{1}(2 c+z)\right]$

$\alpha$

$\operatorname{Re}[\beta$,

$\operatorname{Re}\left[\alpha\left(\beta_{1}-\alpha\right)\right]$

$J\left(r_{1}, 0\right)$

$J\left(r_{2}, 0\right)$

$\alpha \ell$

$\exp [\operatorname{Re}(\alpha, z)]$ 


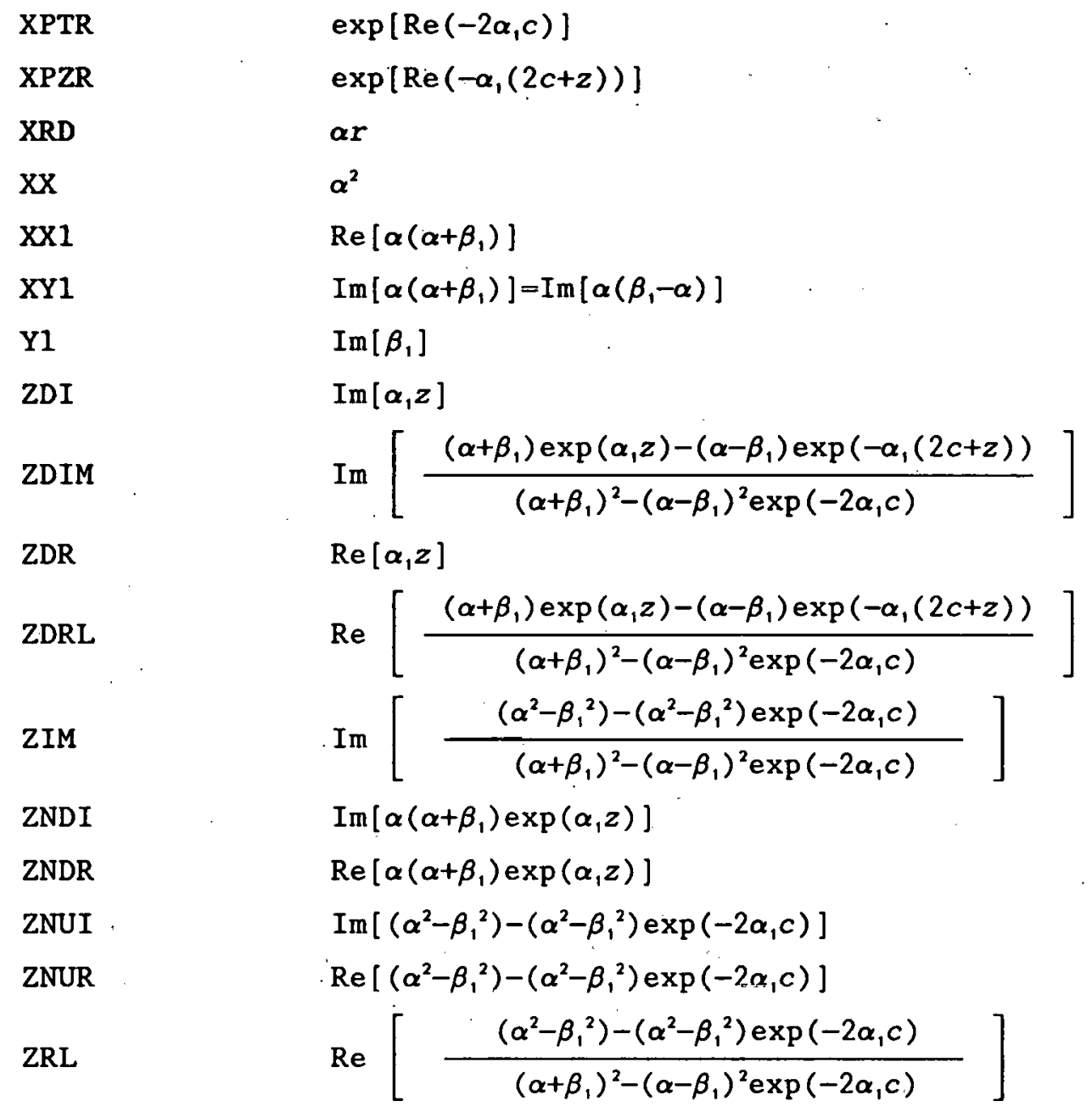

\section{Notes for the integration section}

I1. A number of the variables in the integration section are not always assigned their exact values but are approximated in certain cases to save time. For example, rather than calculate the exponential of a very small number, the Maclaurin series expansion is sometimes used. Also, the exponential of a very large negative number is usually treated as zero.

12. Several variables appear in the integration section of the program which play no part in the calculations being done. They are merely there to do such things as to determine the maximum step size which can be used while still accurately calculating the integrals.

I3. Variables AVZDFR(NR) and AVZDFI(NR) are the averages of the elements in arrays SMZDFR(NZ,NR) and SMZDFI(NZ,NR), respectively, summed over NZT. For improved accuracy, the defect is divided into NZT parts over the depth of the defect, to perform the calculations. The NZT elements of each 
array contain the calculations for these NZT parts. In addition, the defect is divided into NRTT parts in the radial direction. The contributions from these elements are averaged to give the total effect of all of these parts on the impedances of the coils.

14. The variable FZD is the normalized depth from the near side of the plate to the center of the part of the defect with which the program is working. It is not the depth of the center of the actual defect.

\section{Sample output}

Partial printer output of program PCAVVSCN:

PCAVVSCN 20 POINTS USED FAR SIDE DEFECT TIME 9: 1:43 DATE 8/9/89

IN RAD OT RAD LENGTH 0-LIFTOFF L.O. VAR CLADTH DF DEP

$\begin{array}{lllllllr}\text { ACT } & 0.1000 & 0.4100 & 0.1000 & 0.0100 & 0.0200 & 0.2540 & -0.2210 \\ \text { NOR } & 0.3922 & 1.6078 & 0.3922 & 0.0392 & 0.0784 & 0.9961 & -0.8667\end{array}$

RBAR 0.2550 FREQ $=5.000000 E+02$ RHO $=4.0900$ PERM $=1.000$ WUSRR $=4.0493$

NORM IMPD:RL 0.155213 IM 0.833067 AIR IND 6.252919E-03 VOLN 5.1590E-01 LIFT-OFF PHASE $=140.00$
$R$ (NOR) MAG PHASE
$\begin{array}{lll}0.010 & 0.001331 & 93.93\end{array}$
$0.030 \quad 0.001343 \quad 93.93$
$\begin{array}{lll}0.050 & 0.001358 & 93.92\end{array}$
$\begin{array}{lll}0.070 & 0.001380 & 93.91\end{array}$
$0.090 \quad 0.001409 \quad 93.90$
$0.110 \quad 0.001446 \quad 93.88$
$0.130 \quad 0 \quad 0 n 1490 \quad 93.86$
$\begin{array}{lll}0.150 & 0.001541 & 93.84\end{array}$
$\begin{array}{llll}0.170 & 0.001601 & 93.81\end{array}$
$\begin{array}{llll}0.190 & 0.001668 & 93.78\end{array}$ 


\section{Listing}

\section{PROGRAM PCAVVSCN}

C VERSION August 9, 1989

C PROGRAM TO CALCULATE THE NORMALIZED IMPEDANCE CHANGE FOR A FLAT

C BOTTOM HOLE IN A SINGLE PLANAR CONDUCTOR. THE DEFECT IS

C CALCULATED AT AN ARRAY OF POINTS RATHER THAN A SINGLE POINT

C THE DEFECT SIGNAL IS AVERAGED OVER BOTH THE R AND $Z$ :DIMENSIONS.

C THE CALCULATIONS ARE STEPPED IN THE R DIRECTION, AS THE DEFECT IS C SCANNED BY THE PROBE.

$\begin{array}{llllllllllllllllll}\text { C DIA NS } & .221 & .188 & .158 & .129 & .097 & .076 & \text { FS } & .222 & .189 & .158 & .129 & .098 & .077\end{array}$

$\begin{array}{llllllllllllllllllll}\text { C DEP NS } & .221 & .188 & .157 & .128 & .096 & .078 & \text { FS } & .221 & .188 & .156 & .128 & .096 & .078\end{array}$ CHARACTER SIDE $(2) * 4$ IMPLICIT REAL $* 8$ (A-H,O-Z)

REAL $* 8$ L, L1, L2 DIMENSION DFDIAM(12), DFDEP(12), NSIDE (12)

DIMENSION $S 1(6), S 2(6), \operatorname{ERR}(6), R J(200)$

DIMENSION $\operatorname{SMZDFR}(20,200), \operatorname{SMZDFI}(20,200), \operatorname{AVZDFR}(200), \operatorname{AVZDFI}(200)$

DATA LOU/8/,LOD/9/,PI/3.141592653/,NZT/20/,NDF/7/,NRT/150/

DATA $\mathrm{S} 1 / .005, .02, .05, .1, .5,2 . /$

DATA S2/1.0,2.0,5.0,10.0,50.0,200.0/

DATA ERR/.1,.01,.001,1.E-4,1.E-5,1.E-6/

DATA FREQ/500./,RHO1/4.09/,U1/1.0/

DATA TRN/800./,T1/0.254/,SIDE/'NEAR',' FAR'/,DELTAR/0.02/

DATA DFDIAM/

$* .221, .188, .158, .129, .097, .076, .222, .189, .158, .129, .098 ; .077 /$

DATA DFDEP/

$* .221, .188, .157, .128, .096, .078, .221, .188, .156, .128, .096, .078 /$

DATA NSIDE $/ 1,1,1,1,1,1,2,2,2,2,2,2$ /

$\mathrm{NS}=\mathrm{NS}$ IDE $(\mathrm{NDF})$

C TIME AND DATE ARE PRINTED

CALL GETTIM(IHR, IMN, ISE, IFR)

CALL GETDAT (IYR, IMO, IDA)

$I Y R=I Y R-1900$

WRITE (LOU, 2) NZT, SIDE(NS), IHR, IMN, ISE, IMO, IDA, IYR

2 FORMAT ('PCAVVSCN' , I3,' POINTS USED ',A4,' SIDE DEFECT',

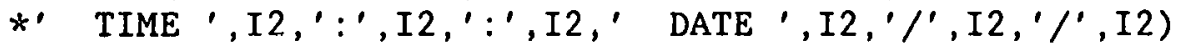

WRITE (LOU, 5)

5 FORMAT(5X, 'IN RAD' , 4X, 'OT RAD' , 4X, 'LENGTH' , 2X, '0-LIFTOFF'

C

*, 2X, 'L.O. VAR', 3X, 'CLADTH', 4X,' DF DEP')

COIL P255A

$\mathrm{R} 1=0.100$

$\mathrm{R} 2=0.410$

$\mathrm{L}=0.100$

C

COIL P371A R $1=0.275 \quad \mathrm{R} 2=0.4665 \quad \mathrm{~L}=0.265$
$\mathrm{~L} 1=0.01$
$\mathrm{~L} 2=0.02$
$\mathrm{R} 3=.5 *(\mathrm{R} 1+\mathrm{R} 2)$
$\mathrm{ZD}=-\mathrm{DFDEP}(\mathrm{NDF})$
$\mathrm{WRITE}(\mathrm{LOU}, 10) \mathrm{R} 1, \mathrm{R} 2, \mathrm{~L}, \mathrm{~L} 1, \mathrm{~L} 2, \mathrm{~T} 1, \mathrm{ZD}$
$\mathrm{R} 1=\mathrm{R} 1 / \mathrm{R} 3$




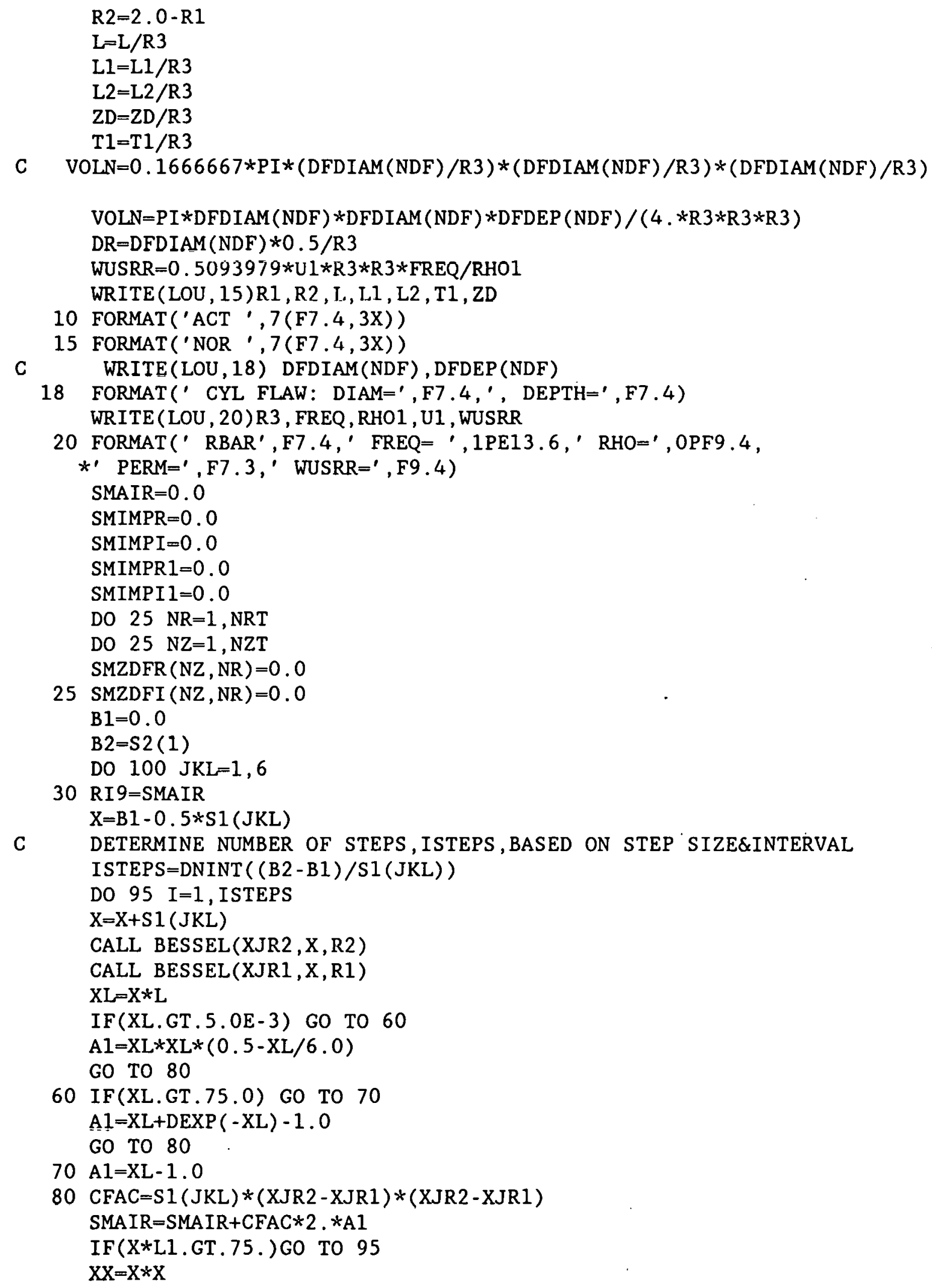


$\mathrm{XI}=\mathrm{DSQRT}(0.5 *(\mathrm{XX}+\mathrm{DSQRT}(\mathrm{XX} * \mathrm{XX}+\mathrm{WUSRR} * \mathrm{WUSRR}))) / \mathrm{U} 1$

$\mathrm{Y} 1=\mathrm{WUSRR} /(2, * \mathrm{X} 1 * \mathrm{U} 1 * \mathrm{U} 1)$

$\mathrm{A} 2=\mathrm{XL}-\mathrm{A} 1$

$A 3=\operatorname{DEXP}(-X * \mathrm{~L} 1)$

$A 4=\operatorname{DEXP}(-X * L 2)$

$\mathrm{APBR}=(\mathrm{X}+\mathrm{X} 1) *(\mathrm{X}+\mathrm{X} 1)-\mathrm{Y} 1 * \mathrm{Y} 1$

$\mathrm{APBI}=2 . * \mathrm{Y} 1 *(\mathrm{X}+\mathrm{X} 1)$

$\mathrm{AMBR}=(\mathrm{X}-\mathrm{X} 1) *(\mathrm{X}-\mathrm{X} 1)-\mathrm{Y} 1 * \mathrm{Y} 1$

$\mathrm{AMBI}=-2 . * \mathrm{Y} 1 *(\mathrm{X}-\mathrm{X} 1)$

$\mathrm{A} 2 \mathrm{BR}=0.0$

$\mathrm{A} 2 \mathrm{BI}=-2 . * \mathrm{X} 1 * \mathrm{Y} 1$

$\mathrm{ZNUR}=\mathrm{A} 2 \mathrm{BR}$

$\mathrm{ZNUI}=\mathrm{A} 2 \mathrm{BI}$

$\mathrm{DENR}=\mathrm{APBR}$

$\mathrm{DENI}=\mathrm{APBI}$

$D N C J=D E N R * D E N R+D E N I * D E N I$

C

SECTION THAT MULTIPLIES BY DEXP(ALPHAI*ZDEFECT)

DO $91 \mathrm{NZ}=1$, NZT

C NEAR SIDE DEFECT CALCULATION

$F Z D=(F L O A T(N Z)-.5) * Z D / F L O A T(N Z T)$

C FAR SIDE DEFECT CALCULATION

IF (NS .EQ . 2) FZD $=-T 1-F Z D$

$\mathrm{ZDR}=\mathrm{X} 1 * \mathrm{U} 1 * \mathrm{FZD}$

IF(ZDR.LT . -60.0)GO TO 93

$\mathrm{ZDI}=\mathrm{Y} 1 * \mathrm{U} 1 * \mathrm{FZD}$

$\mathrm{XPDR}=\mathrm{DEXP}(\mathrm{ZDR})$

$\mathrm{CSDI}=\mathrm{DCOS}(\mathrm{ZDI}) * \mathrm{XPDR}$

$\operatorname{SNDI}=\mathrm{DSIN}(\mathrm{ZDI}) * X P D R$

$\mathrm{XX} 1=\mathrm{X} * \mathrm{X} 1+\mathrm{XX}$

$\mathrm{XY} 1=\mathrm{X} * \mathrm{Y} 1$

$\mathrm{X} 1 \mathrm{X}=\mathrm{X} * \mathrm{X} 1-\mathrm{XX}$

$\mathrm{ZNDR}=\mathrm{XX} 1 * \mathrm{CSDI}-\mathrm{XY} 1 * \mathrm{SNDI}$

$\mathrm{ZNDI}=\mathrm{XX} 1 * \mathrm{SNDI}+\mathrm{XY} 1 * \mathrm{CSDI}$

C SECTION THAT MULTIPLIES BY DEXP(ALPHAI* $(2 * T H+Z D E F E C T)$ )

$\mathrm{TZR}=\mathrm{X} 1 * \mathrm{U} 1 *(2 . * \mathrm{~T} 1+\mathrm{FZD})$

IF(TZR.GT.60.)GO TO 87

$\mathrm{TZI}=\mathrm{Y} 1 * \mathrm{U} 1 *(2 . * \mathrm{~T} 1+\mathrm{FZD})$

$\mathrm{XPZR}=\mathrm{DEXP}(-\mathrm{TZR})$

$\mathrm{CSZI}=\mathrm{DCOS}(\mathrm{TZT}) * X P Z R$

$S N Z I=D S I N(T Z I) * X P Z R$

$\mathrm{ZNDR}=\mathrm{XX} 1 * \mathrm{CSDI}-\mathrm{XY} 1 * \mathrm{SNDI}+\mathrm{X} 1 \mathrm{X} * \mathrm{CSZI}+\mathrm{XY} 1 * \mathrm{SNZI}$

$\mathrm{ZNDI}=\mathrm{XX} 1 * \mathrm{SNDI}+\mathrm{XY} 1 * \mathrm{CSDI}+\mathrm{XY} 1 * \mathrm{CSZI}-\mathrm{X} 1 \mathrm{X} * \mathrm{SNZI}$

C SECTION THAT MULTIPLIES BY DEXP (-ALPHA $1 * 2 *$ CLADTH)

$\mathrm{TR}=2 . * \mathrm{X} 1 * \mathrm{U} 1 * \mathrm{~T} 1$

IF(TR.GT.60.) GO TO 87

$\mathrm{TI}=2 . * \mathrm{Y} 1 * \mathrm{U} 1 * \mathrm{~T} 1$

$\mathrm{XPTR}=\mathrm{DEXP}(-\mathrm{TR})$

$\operatorname{CSTI}=\mathrm{DCOS}(\mathrm{TI}) * \mathrm{XPTR}$

SNTI $=$ DSIN $(T I) * X P T R$

$\mathrm{DENR}=\mathrm{APBR}-\mathrm{AMBR} * \mathrm{CSTI}-\mathrm{AMBI} * \mathrm{SNTI}$

$\mathrm{DENI}=\mathrm{APBI}-\mathrm{AMBI}+\mathrm{CST} I+\mathrm{AMBR} * \mathrm{SNTI}$ 


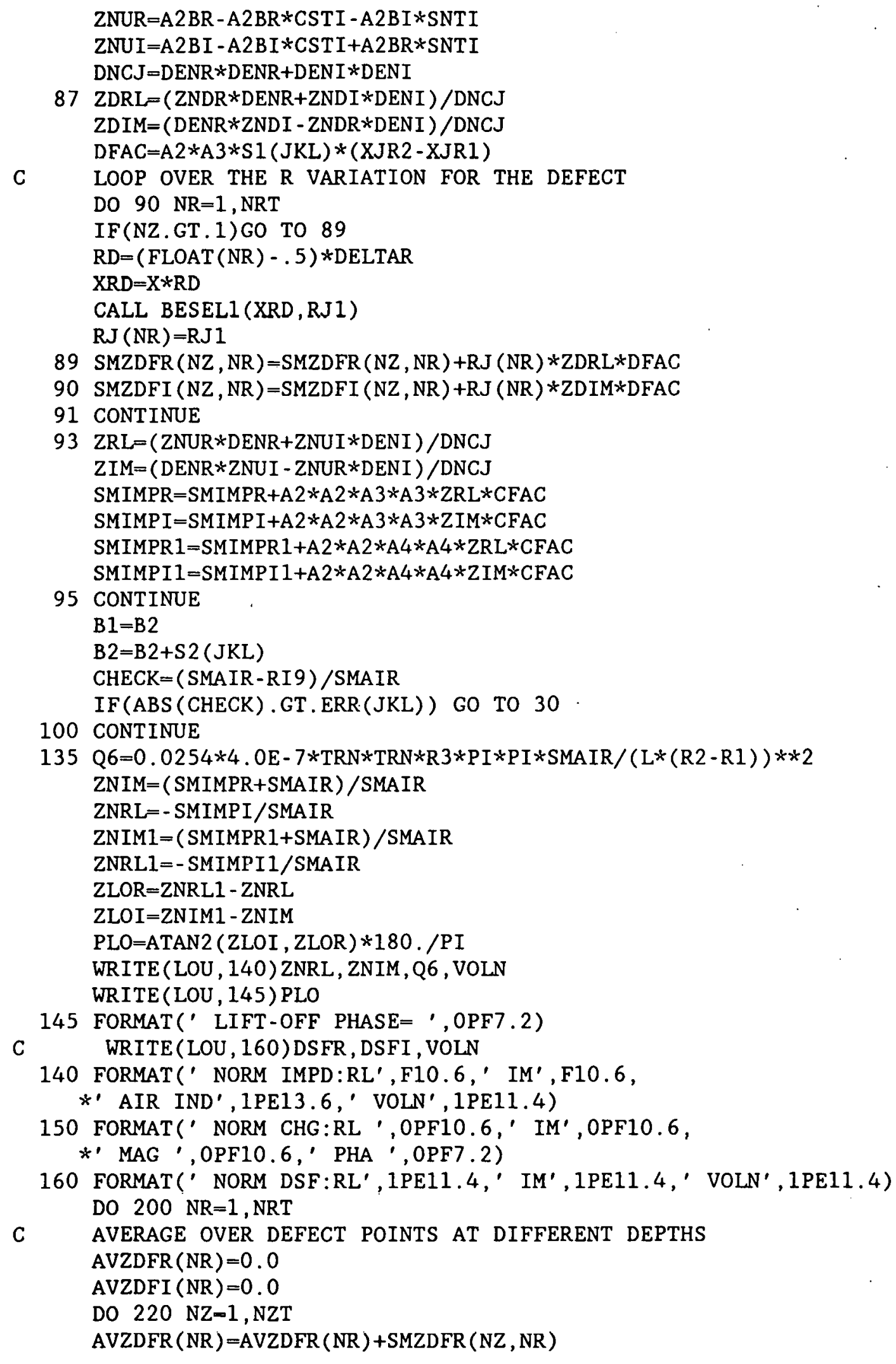


$220 \operatorname{AVZDFI}(\mathrm{NR})=\operatorname{AVZDFI}(\mathrm{NR})+\operatorname{SMZDFI}(\mathrm{NZ}, \mathrm{NR})$

$\operatorname{AVZDFR}(\mathrm{NR})=\operatorname{AVZDFR}(\mathrm{NR}) /$ FLOAT $(\mathrm{NZT})$

$\operatorname{AVZDFI}(N R)=\operatorname{AVZDFI}(\mathrm{NR}) /$ FLOAT $(N Z T)$

200 CONTINUE

C AVERAGE OVER THE DEFECT AT DIFFERENT R VALUES

C SD=LOCATION OF DEFECT CENTER

C RD=FIELD POINT WHERE DEFECT IMPEDANCE CHANGE IS CALCULATED

C DR=DEFECT RADIUS - ALL DIMENSIONS ARE NORMALIZED

WRITE (LOU, *) ' R(NOR) MAG PHASE'

$\mathrm{NDT}=2.0 * \mathrm{DR} / \mathrm{DELTAR}$

$D R R=F L O A T(N D T) * D E L T A R * 0.5$

C START SD AT 0 IF NDT EVEN, 1ST HALF STEP IF NDT ODD ."

$S D=F L O A T(M O D(N D T, 2)) * .5 * D E L T A R$

NRTT $=2 *$ NRT $/ 3$

DO $400 \mathrm{NR}=1$, NRTT

AVZDR $=0.0$

AVZDI $=0.0$

SUML $=0.0$

$\mathrm{RD}=\mathrm{SD}-\mathrm{DRR}+\mathrm{DELTAR} * .5$

DO $300 \mathrm{ND}=1$, NDT

$\mathrm{AL}=0.0$

IF (RD.LT.0.0)GO TO 290

$\mathrm{NRD}=(0.50001+\mathrm{RD} / \mathrm{DELTAR})$

IF (RD . LT.DR-SD) $\mathrm{AL}=2 . * 3.14159 * \mathrm{RD}$

IF (RD.LT.DR-SD) GO TO 280

$\mathrm{ANG}=(\mathrm{SD} * \mathrm{SD}+\mathrm{RD} * \mathrm{RD}-\mathrm{DR} * \mathrm{DR}) /(2 . * \mathrm{RD} * \mathrm{SD})$

$\mathrm{AL}=2 . * \mathrm{RD} * \mathrm{ACOS}$ (ANG)

280 CONTINUE

$290 \mathrm{RD}=\mathrm{RD}+\mathrm{DELTAR}$

SUML=SUML+AL

$A V Z D R=A V Z D R+A V Z D F R(N R D) * A L$

$A V Z D I=A V Z D I+A V Z D F I(N R D) * A L$

300 CONTINUE

AVZDR=AVZDR/SUML

AVZDI $=A V Z D I / S U M L$

$\mathrm{DSFR}=-1.5 *$ WUSRR $*(A V Z D R * A V Z D R-A V Z D I * A V Z D I) /(S M A I R * P I)$

$D S F I=-1.5 * W U S R R * 2.0 * A V Z D R * A V Z D I /(S M A I R * P I)$

$\mathrm{ZNDFR}=\mathrm{VOLN} * \mathrm{DSFR}$

$\mathrm{ZNDFI}=\mathrm{VOLN} * \mathrm{DSFI}$

$\mathrm{DFM}=\mathrm{VOLN} * \mathrm{SQRT}(\mathrm{DSFR} * \mathrm{DSFR}+\mathrm{DSFI} * \mathrm{DSFI})$

$\mathrm{DFP}=\mathrm{ATAN} 2(\mathrm{DSFI}, \mathrm{DSFR}) \times 180 . / \mathrm{PI}$

WRITE (LOU, 380) SD , DFM, DFP

WRITE (LOD , 390) DFM , DFP

380 FORMAT (F7. 3, F10.6,F7.2)

390 FORMAT (E12.5, F7.2)

$S D=S D+D E L T A R$

400 CONTINUE

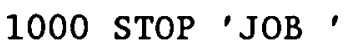

END 
PCGRAPH plots two sets of data on same graph

Program PCGRAPH plots two sets of data on the same graph and sends the output to the screen and the printer. It is normally used to compare the calculated and the experimental values of the impedance change of a pancake coil due to a defect at different distances from the defect. The initial plot is made on the CRT and then the program sends the data on the screen to the printer.

\section{Summary}

1. Dimension arrays and declare variabile types.

2. Initialize variables.

3. Open the files containing the data:.

4. Read in the data and convert them to: a: form usable by the graphics subroutines.

5. Graph the data on the screen.

6. Send the contents of the screen to the printer.

\section{Variables}

CX An array containing the real parts of one set of the data to be plotted.

$\mathrm{CY} \quad$ An array containing the imaginary: parts of one set of the data to be plotted.

DFDEP $\quad$ The depth to the bottom of the defect in inches.

DFDIAM $\quad$ The diameter of the defect in inches.

EX An array containing the real parts of one set of the data to be plotted.

EY An array containing the imaginary parts of one set of the data to be plotted.

FF Character variable containing the form-feed character.

FREQ $\quad$ The operating frequency in hertz.

GIM Factor by which the imaginary parts of the data to be graphed are multiplied to make the graphs as large as possible.

GRL Factor by which the real parts of the data to be graphed are multiplied to make the graphs as large as possible.

$L^{*} \quad$ The length of the coil. The value is input in inches and normalized by the program.

L1 The lift-off of the coil. The value is input in inches and normalized by the program.

LOEC The number of the I/O unit connected to the file containing the calculated data.

LOEE $\quad$ The number of the I/O unit connected to the file containing the experimental data.

LOU The number of the $I / O$ unit connected to the printer. 


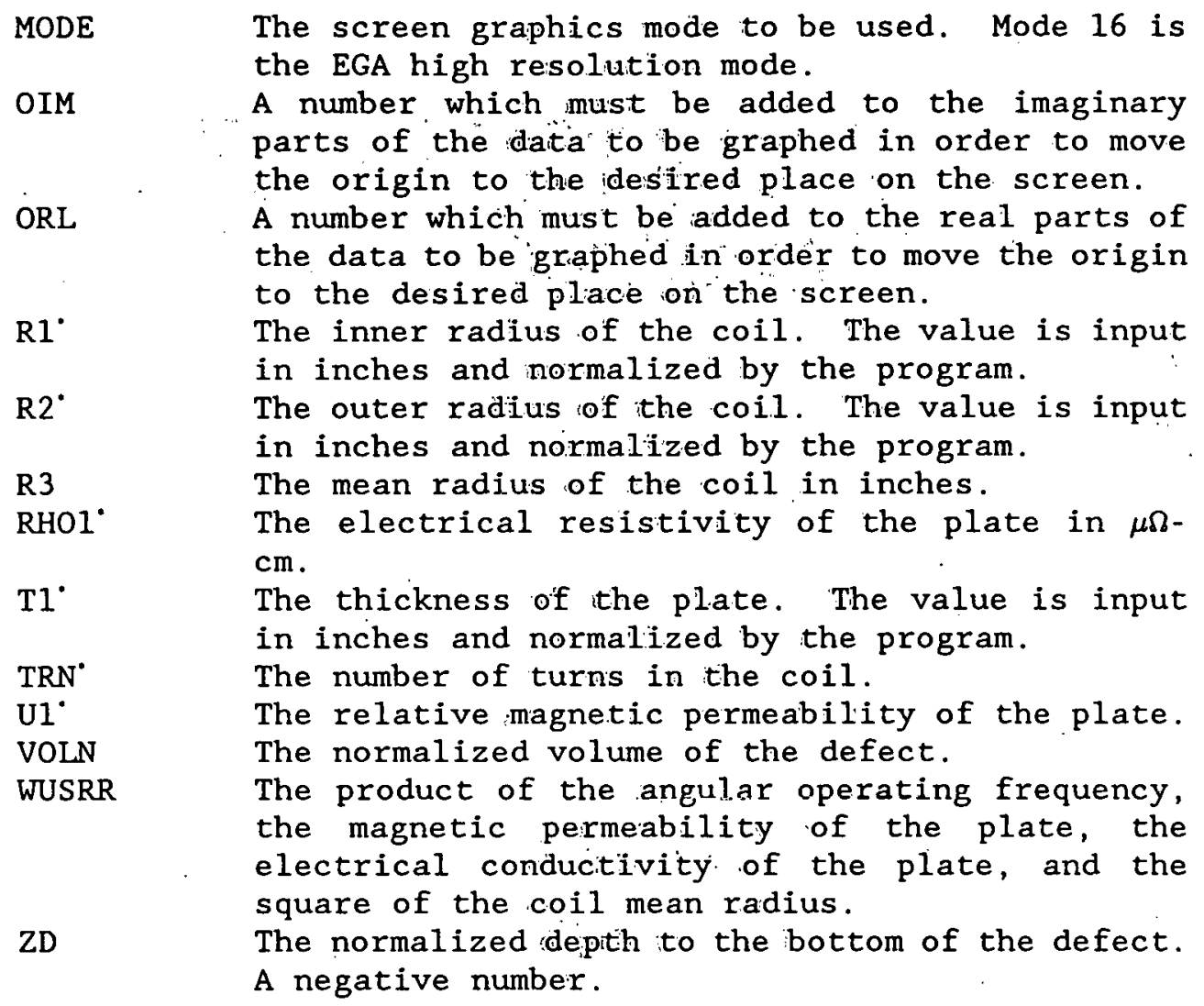




\section{Listing}

FROGRAM PCGRAPH

CHARACTER $* 1$ FF

DIMENSION CX(200),CY(200), EX(200), EY(200)

REAL GRL, GIM, L, LI

DATA LOU/8/,LOEE/38/, LOEC/39/

DATA PI $/ 3.141592653 /, \mathrm{MODE} / 16 /$

DATA ORL $/ 330 /, 0$ IM $/ 50 /$

DATA FREQ/500./,RHO1/4.054/,U1/1.0/,TRN/800./

DATA DFDIAM/0.1881/,DFDEP/0.1881/

$F F=$ CHAR (12)

CALL GETTIM(IHR, IMN, ISE, IFR)

CALL GETDAT (IYR, IMO, IDA)

$I Y R=I Y R-1900$

WRITE (LOU , 2) IHR, IMN, ISE, IMO, IDA, IYR

2 FORMAT(' PCGRAPH TIME', I2, ':', I2, ' :', I2

*,' DATE ', I2,' $/$ ', I2,' $/$ ', I2, ()

OPEN (LOEE , FILE $=$ ' QEXPN2. DAT' , STATUS=' OLD' )

OPEN (LOEC, FILE=' OCALN2. DAT' , STATUS=' OLD' $)$

WRITE (LOU, *)' NEAR SIDE DFFECT'

WRITE (LOU , 5)

5 FORMAT( $5 \mathrm{X}$,' IN RAD', 4X, 'OT RAD', 4X, 'LENGTH' ,4X, 'LIFTOFF'

$*, 3 \mathrm{X},{ }^{\prime}$ CLADTH' $4 \mathrm{X}$, 'DF DEP' , 4X, 'DFDIAM')

$\mathrm{R} 1=0.1000$

$\mathrm{R} 2=0.4100$

$\mathrm{L}=0.1000$

$\mathrm{L} 1=0.010$

$\mathrm{T} 1=0.250$

$\mathrm{R} 3=0.5 *(\mathrm{R} 1+\mathrm{R} 2)$

$\mathrm{ZD}=-\mathrm{DFDEP}$

WRITE (LOU , 10)R1 , R2 , L , L1 , T1 , ZD , DFDIAM

10 FORMAT ('ACT:' , 7(F7.4,3X))

$\mathrm{R} 1=\mathrm{R} 1 / \mathrm{R} 3$

$\mathrm{R} 2=2.0-\mathrm{R} 1$

$\mathrm{L}=\mathrm{L} / \mathrm{R} 3$

$\mathrm{L} 1-\mathrm{L} 1 / \mathrm{R} 3$

$\mathrm{TI}=\mathrm{T} 1 / \mathrm{R} 3$

$\mathrm{ZD}=\mathrm{ZD} / \mathrm{R} 3$

DFDIAM $=$ DFDIAM $/$ R 3

WRITE (LOU , 15) R1, R2 , L , L1 , T1 , ZD , DFDIAM

15 FORMAT ('NOR:' , 7(F7.4,3X))

VOLN $=$ PI $*$ DFDIAM $*$ DFDIAM $*$ DFDEP $/(4 * * \mathrm{R} 3 * \mathrm{R} 3 * \mathrm{R} 3)$

WUSRR $-0.5093979 * U 1 * R 3 * R 3 * F R E Q / R H O 1$

WRITE (LOU , 18)R3, RHO1, U1, WUSRR

18 FORMAT(' RBAR', F7.4,' RHO=', OPF7.4,

*' PERM=',F7.3,' WUSRR=',F9.4)

CALL QSMODE (MODE)

CALL GRID

11 CXMAX $=0$.

$C Y M A X=0$. 
$\operatorname{EXMAX}=0$.

EYMAX $=0$.

EMMAX $=0$.

CMMAX $=0$.

$I=1$

20 READ (LOEE , *, END=29) EMAG , EPHA

$E P H A=E P H A * P I / 180$.

$\operatorname{EX}(I)=E M A G * \operatorname{COS}(E P H A)$

$\operatorname{EY}(I)=E M A G * S I N(E P H A)$

$\operatorname{IF}(\operatorname{EX}(I) . G T$. EXMAX) EXMAX $=E X(I)$

IF (EY (I) . GT . EYMAX) EYMAX $=E Y(I)$

IF(EMAG.GT . EMMAX) THEN

EMMAX =EMAG

EPMMAX $=E P H A$

END IF

$I=I+1$

GO TO 20

$29 \operatorname{EX}(\mathrm{I})=999$.

$I=1$

30 READ ( EOEC, *, END=40) CMAG, CPHA

$\mathrm{CPHA}=\mathrm{CPHA} * \mathrm{PI} / 180$.

$C X(I)=C M A G * \operatorname{COS}(C P H A)$

CY (I) $=$ CMAG $*$ S IN $($ CPHA $)$

IF $(\mathrm{CX}$ (I) . GT . CXMAX) $\mathrm{CXMAX}=\mathrm{CX}$ (I)

IF (CY(I) . GT . CYMAX) CYMAX $=C Y$ (I)

IF.(CMAG..GT. CMMAX) THEN

GMMAX $=$ CMAG

GPMMAX = CPHA

END IF

$\mathrm{I}=\mathrm{I}+1$

GO TO 30

$40 \mathrm{CX}(\mathrm{I})=999$.

$\operatorname{EGIM}=300 . / \mathrm{EXMAX}$

EGRL $=300 . /$ EYMAX

IF(EGIM.GT.EGRL) THEN

EGIM=EGRL

ELSE

EGRL=EGIM

END IF

CGIM $=300 . / \mathrm{CXMAX}$

CGRL $=300 . /$ CYMAX

IF.(CGIM.GT. CGRL) THEN

CGIM=CGRL

ELSE

CGRL $=$ CGIM

END IF

IF(EGIM.GT,CGIM) THEN

$G I M=C G I M$

$\mathrm{GRL}=\mathrm{CGRL}$

ELSE

(GIM=EGIM 


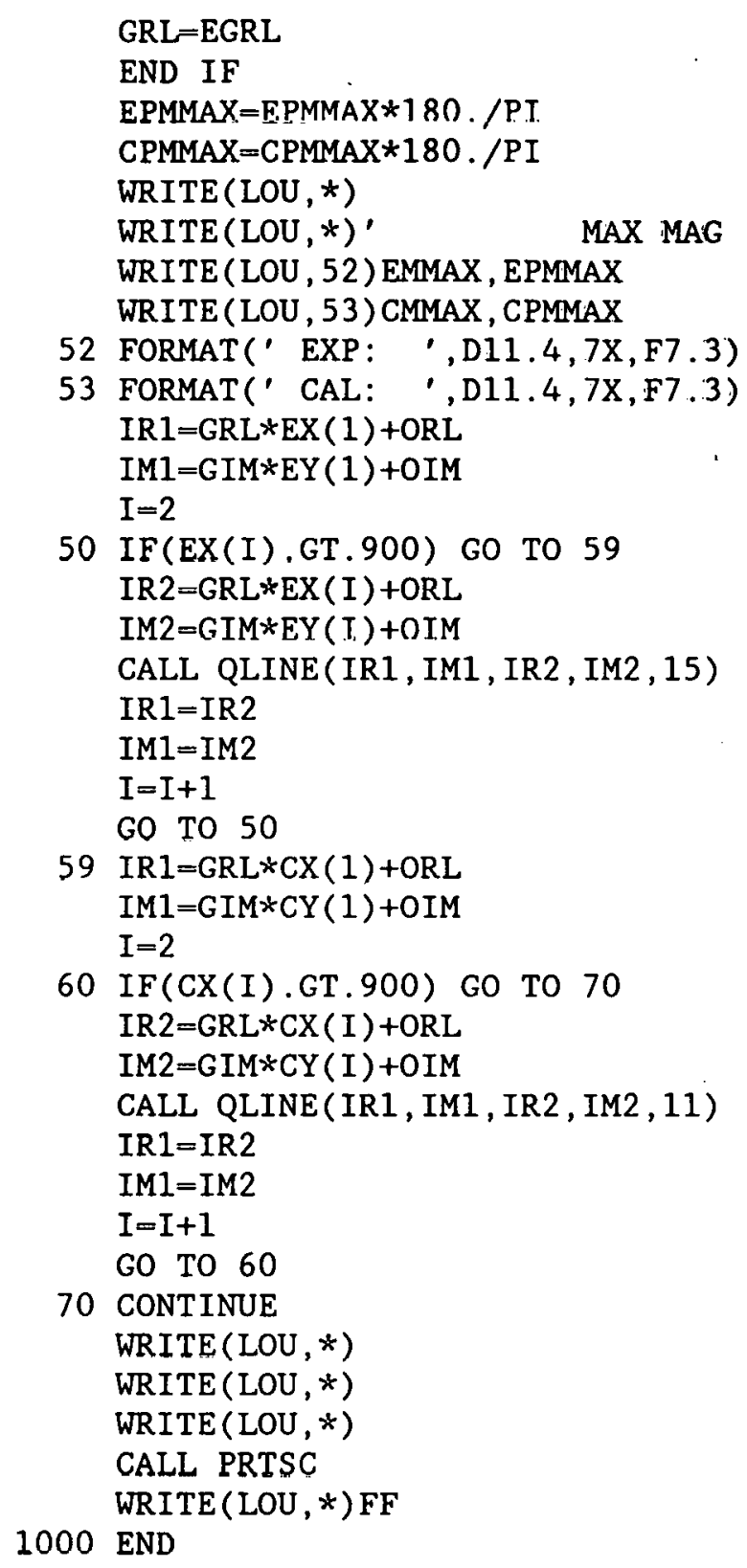


PCINV Inverts scan of pancake coll data to get depth and volume

Program PCINV calculates the depth and volume of a defect given the change in the impedance of a pancake coil as it scans past the defect. The program works with experimental data stored in a file by program PCFIX or with calculated data stored by program PCAVZSCN, and it uses a lookup file built by program PCBLDF. The program calculates the integral of minus the impedance change in the coil due to the defect with respect to the radial distance between the coil axis and the defect from the inner radius of the coil to the outer radius of the coil. It then compares the phase of this complex integral with the phases stored in a lookup file by program PCBLDF. When the phase of the integral matches the phase in the lookup file, the program reads the corresponding depth and magnitude from the lookup file. The depth is equal to the depth of the defect, and the magnitude can be used to find the volume of the defect. The defect is assumed to have the shape of a flat-bottomed hole.

\section{Summary}

1. Declare variable types.

2. Initialize variables.

3. Open the file containing the experimental data, read in the data, and calculate the integral.

4. Find the depth and magnitude in the lookup file corresponding to the phase of the integral.

5. Calculate the inverted defect depth and volume based on the experimental data.

6. Open the file containing the calculated data, read in the data, and calculate the integral.

7. Find the depth and magnitude in the lookup file corresponding to the phase of the integral.

8. Calculate the inverted defect depth and volume based on the calculated data.

\section{Variables}

$\begin{array}{ll}\text { AIRIND: } & \text { The inductance in henries of the coil in air. } \\ \text { DELRDC } & \text { The normalized distance between adjacent } \\ \text { calculated data points. } \\ \text { The normalized distance between adjacent } \\ \text { experimental data points. } \\ \text { The inverted depth of the center of the defect. } \\ \text { DEPTH } \\ \text { A negative number. } \\ \text { DFDEP } & \text { The actual depth of the defect in inches. } \\ \text { DFDIAM: } & \text { The actual diameter of the defect in inches. } \\ \text { DFM } & \text { The magnitude of the change in the impedance of } \\ \text { DFP. } & \text { The coil due to the defect. } \\ \text { The phase of the change in the impedance of the }\end{array}$


$\mathrm{L}^{\circ}$

$\mathrm{LI}^{\circ}$

LHSPHA

LOEC ${ }^{*}$

LOEE ${ }^{*}$

LOU

$\mathrm{R} 1^{\circ}$

R2 ${ }^{\circ}$

R3

RDC

RDE

RHOI $^{\circ}$

RHSMAG

SMAIR

$\mathrm{T}^{\circ}$

TRN"

$\mathrm{U} 1{ }^{\circ}$

VOL1

VOLN

WUSRR

XMAG

ZD
The length of the coil. The value is input in inches and normalized by the program.

The lift-off of the coil. The value is input in inches and normalized by the program.

The phase of the integral calculated by the program.

The number of the $I / 0$ unit connected to the file containing the calculated data.

The number of the $I / O$ unit connected to the file containing the experimental data.

The number of the $I / O$ unit connected to the printer.

The inner radius of the coil. The value is input in inches and normalized by the program.

The outer radius of the coil. The value is input in inches and normalized by the program.

The mean radius of the coil in inches.

The radial distance between the axis of the coil and the center of the defect in the part of the program which inverts the calculated data.

The radial distance between the axis of the coil and the center of the defect in the part of the program which inverts the experimental data.

The electrical resistivity of the plate in $\mu \Omega$ $\mathrm{cm}$.

The magnitude retrieved from the lookup file.

A quantity related to the inductance of the coil in air. It is used to normalize the impedance of the coil.

The thickness of the plate. The value is input in inches and normalized by the program.

The number of turns in the coil.

The relative magnetic permeability of the plate.

The inverted normalized volume of the defect.

The actual normalized volume of the defect.

The product of the angular operating frequency, the magnetic permeability of the plate, the electrical conductivity of the plate, and the square of the mean radius of the coil.

The magnitude of the integral calculated by the program.

The normalized depth of the center of the defect. A negative number. 


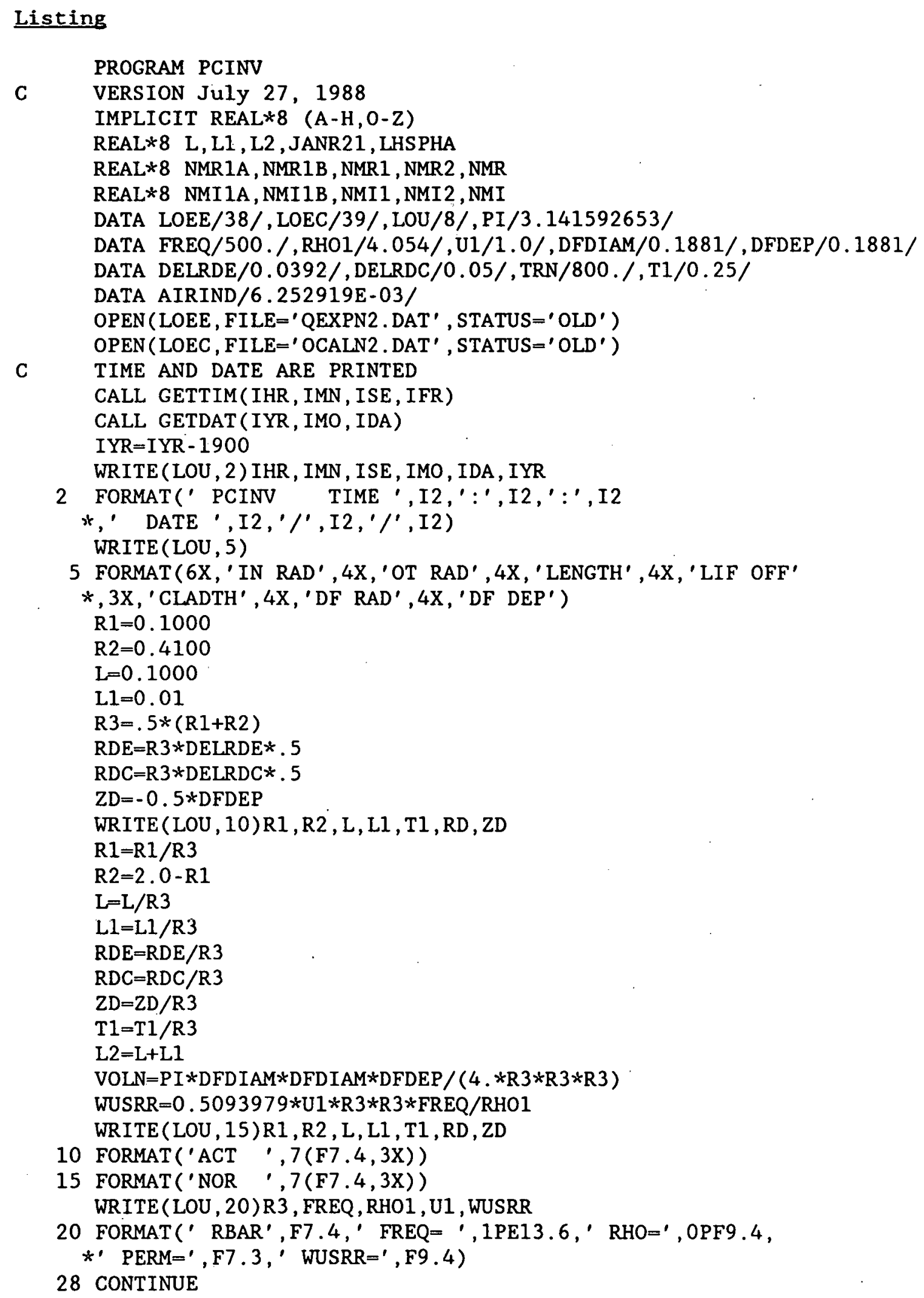




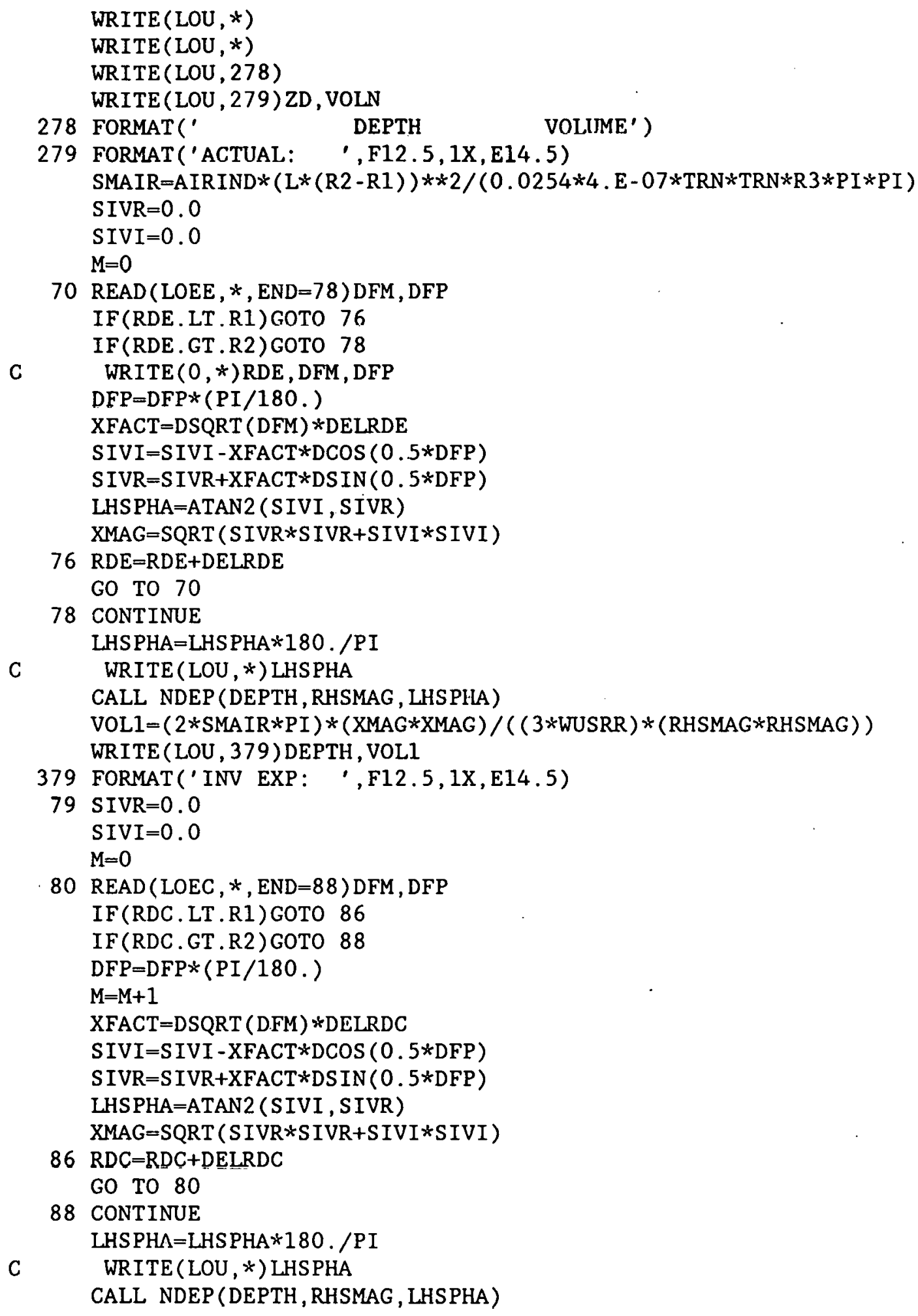


68

VOLI $=(2 *$ SMAIR $* P I) *(X M A G * X M A G) /((3 *$ WUSRR $) *($ RHSMAG*RHSMAG $))$ WRITE (LOU , 479) DEPTH, VOL1

479 FORMAT('INV CAL: ',F12.5, 1X,E14.5)

END 
PCRTSCAN converts raw voltage readings to impedance change

Program PCRTSCAN converts raw experimental voltage readings taken by a pancake coil scanning across a plate into the impedance change in the coil due to a defect. The program can then use this array of impedance changes to locate defects in the plate and to calculate the depth and volume of the defects. The program locates the defects by constructing three windows, a "zero" window and two windows directly under the coil windings. These two windows are located from R1 to R2 on either side of the coil axis, and a running sum of the magnitude of the impedance change, referenced to the zero window, is kept for each of these windows. A dot product is performed between the impedance change in these two windows, and the defect center is located at the maximum value of this product. The "zero" window is shifted so that it will be in a clean region of the sample, but on either side of the defect. In Fig. 6 we show the magnitude of the impedance change plotted for a scan of six defects on the near side of the plate.

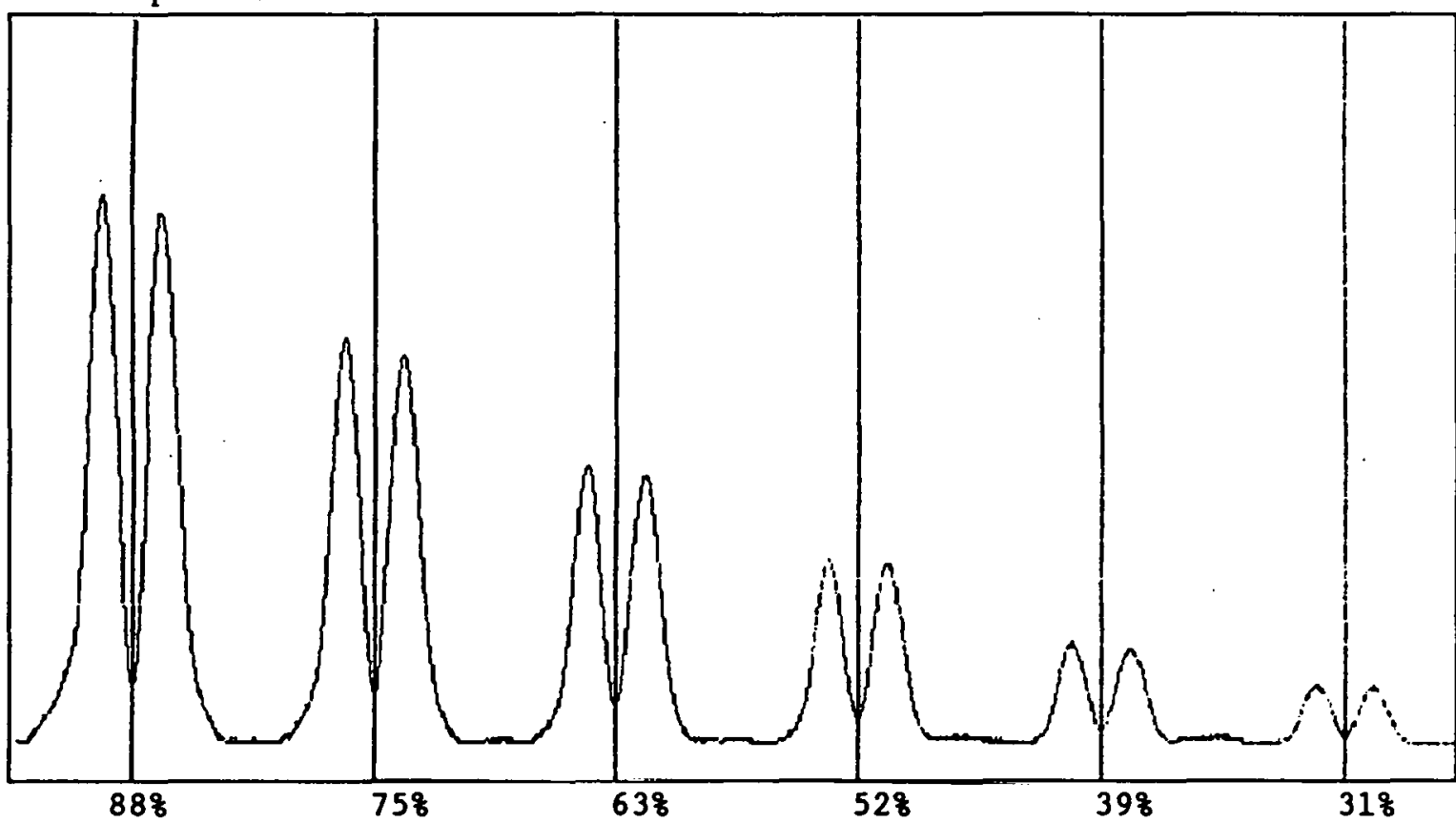

Figure 6 Magnitude of impedance change measured with a pancake coil for near side defects.

The defects have a diameter approximately equal to the depth of the defect, so that the defect volume falls off as the depth cubed. In Fig. 7 we show a similar plot for defects on the far side of the plate. Note that the noise has increased such that the last two defects have not been located using the present noise cut-off level. However, these are very low volume defects compared to those normally detected by eddy-current tests. Relative to the wall thickness, the ASME Section XI 408 standard defect has a volume 94 times greater than the 398 defects in the test 
plate. The defects were chosen to be relatively small so that better agreement would be obtained with the theory.

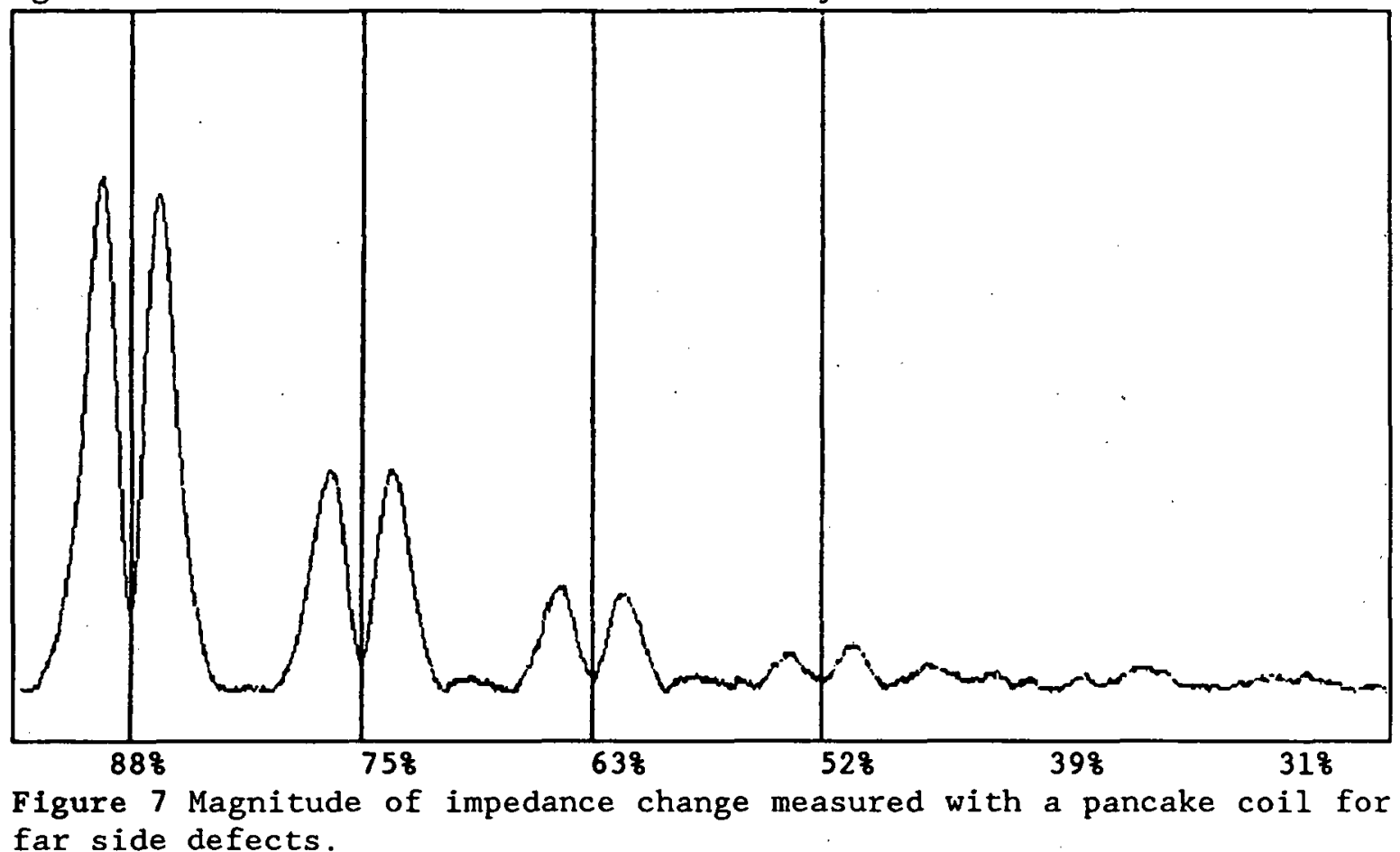

The original version of PCRTSCAN read data directly from voltmeters connected to the MIZ17, but later versions, such as the present listing, read the data taken from the MIZ17 and stored in a data file.

\section{Summary}

1. Dimension arrays and declare variable types.

2. Initialize variables.

3. Get the voltage readings with additional lift-off and convert these readings to the impedance change caused by the additional lift-off.

4. Take enough readings to fill up both of the active windows and the region between the windows.

5. Average the first NZ readings taken on the plate and use this average value as the voltage in the absence of defects.

6. Calculate the integrals in the active windows and solve for the depth and volume of a possible defect centered between the active windows.

7. Check to see if a defect is located between the active windows. If there is a defect, find and record its depth and volume.

8. Advance the zero window one point. Check to see if the entire zero window is out of the range of defects. If it is, average the values in the zero window to find the new value of the voltage in the absence of defects.

9. Advance the active windows one point.

10. Go to 6 until the entire plate has been scanned. 
11. Graph the results.

\section{Variables}

AIRIND*

The inductance of the coil in air.

CLOM $^{\circ}$

The magnitude of the calculated value of the change in the impedance of the coil due to additional lift-off.

CLOP The phase in radians of the calculated value of the change in the impedance of the coil due to additional lift-off.

DEFECT An array which contains the position of each defect located by the program. It contains the distance in inches from each defect to the point where the scan began.

DELR The normalized distance between adjacent data points.

DELTAX The distance between adjacent data points in inches.

DEPA An array containing the inverted depths at each point along the plate.

DP The dot product of the integrals from the two active windows.

FF

A character variable containing the form feed character.

FREQ The operating frequency in hertz.

HA1 The number of lle first data poine in the firot active window.

HA2 The number of the first data point in the second active window.

HZ The number of the first data point in the zero window.

ICP Flag which is set when a defect is located and is reset when the dot product of the integrals in the t.wn astive windows stops decreasing. This flag must be 0 for the program to signal that it has found a defect.

$L^{*} \quad$ The length of the coil. The value is input in inches and normalized by the program.

L1' The lift-off of the coil. The value is input in inches and normalized by the program.

L2 The distance from the top of the coil to the plate.

LA1 The number of the last data point in the first active window.

LA2 The number of the last data point in the second active window.

LHSMAG The magnitude of the average of the integrals from the two active regions.

LHSPHA The phase in degrees of the average of the 
integrals from the two active regions.

LOD $\quad$ The number of the I/O unit connected to the lookup file built by program PCBLDF.

LOE' The number of the I/O unit connected to the file containing the raw experimental data.

LOU The number of the $I / O$ unit connected to the printer.

L2 The number of the last data point in the zero window.

NA

The number of data points in each active window.

ND

NE

The number of defects located by the program.

The number of data points in the region between the active windows.

$\mathrm{NZ}$

$\mathrm{R} 1^{\circ}$

R2 ${ }^{*}$

The number of data points in the zero window.

The inner radius of the coil. The value is input in inches and normalized by the program.

R3 The outer radius of the coil. The value is input in inches and normalized by the program.

RAWI The mean radius of the coil in inches.

RAWR An array containing the imaginary parts of the raw readings taken at each point across the plate.

An array containing the real parts of the raw readings taken at each point across the plate.

$\mathrm{RHO1}^{\circ}$ The electrical resistivity of the plate in $\mu \Omega$ $\mathrm{cm}$.

SACTI The imaginary part of the average of the integrals from the two active regions.

SACTR The real part of the average of the integrals from the two active regions.

SCFAC The ratio of the magnitude of the calculated change in the normalized impedance to the magnitude of the change in the raw reading due to additional lift-off.

SCPHA The difference between the phase of the calculated change in the normalized impedance and the phase of the change in the raw reading due to additional lift-off.

TACTI1 The imaginary part of the integral over the region spanned by the first active window.

TACTI2 The imaginary part of the integral over the region spanned by the second active window.

TACTR1 The real part of the integral over the region spanned by the first active window.

TACTR2 The real part of the integral over the region spanned by the second active window.

TRN $^{*} \quad$ The number of turns in the coil.

$\mathrm{U} 1^{*}$ The relative magnetic permeability of the plate.

VLOI The imaginary part of the experimental reading taken with additional lift-off.

VLOM The magnitude of the experimental reading taken with additional lift-off. 
VLOP The phase of the experimental reading taken with additional lift-off.

VLOR $\quad$ The real part of the experimental reading taken with additional lift-off.

VOLA An array containing the inverted volume at each point along the plate.

VTOL The maximum amount of drift in the readings in the zero window that the program will tolerate for it to re-zero the readings.

WUSRR. The product of the angular frequency, the magnetic permeability, the electrical conductivity, and the square of the mean coil radius.

ZEROI The value of the imaginary part of the voltage on a section of the plate with no defects.

ZEROR The value of the real part of the voltage on a section of the plate with no defects.

ZEROY The difference between the number 20 and the number of data points since the program last found a zero point on the plate. If it has been more than 20 points since the last time the program found a zero, $2 E R O Y=0$.

Notes

1. For the program to signal that it has found a defect, four conditions must be satisfied:

(a) The inversion of the preliminary data must result in a defect which is inside the plate.

(b) The scalar product of the integrals from the two active windows must decrease twice consecutively after having increased.

(c) The scalar product of the integrals from the two active windows must be greater than 4.0E-04.

(d) No zero can have been detected within the last 20 readings.

Some of these criteria may be unnecessary.

2. If the program is too sensitive to zeros, that is, if it finds zeros in places it should not, it can be made less sensitive by increasing the value of NZ or by decreasing the value of VTOL. Conversely, if the program fails to find a zero in a clean region of the plate, it may be made more sensitive to such regions by decreasing NZ or by increasing VTOL.

3. When the program locates a defect, it searches until it finds the next zero on the plate, and it averages the zeros on both sides of the defect. While this makes the inversion more accurate, it also makes it possible that the program will overlook a defect. If there are two defects on the plate which are not separated by a region that the program recognizes as clean, the program will not detect the second defect. 
4. The program averages the first $\mathrm{NZ}$ readings and uses this as the value on the clean part of the plate until it finds a clean section of the plate. If the very first region presented to the program has a defect in it, the results will be unpredictable and very likely undesirable because the program uses the very first reading in its calculation of SCFAC and SCPHA which are used to convert all subsequent readings to the normalized impedance change of the coil. 


\section{Listing}

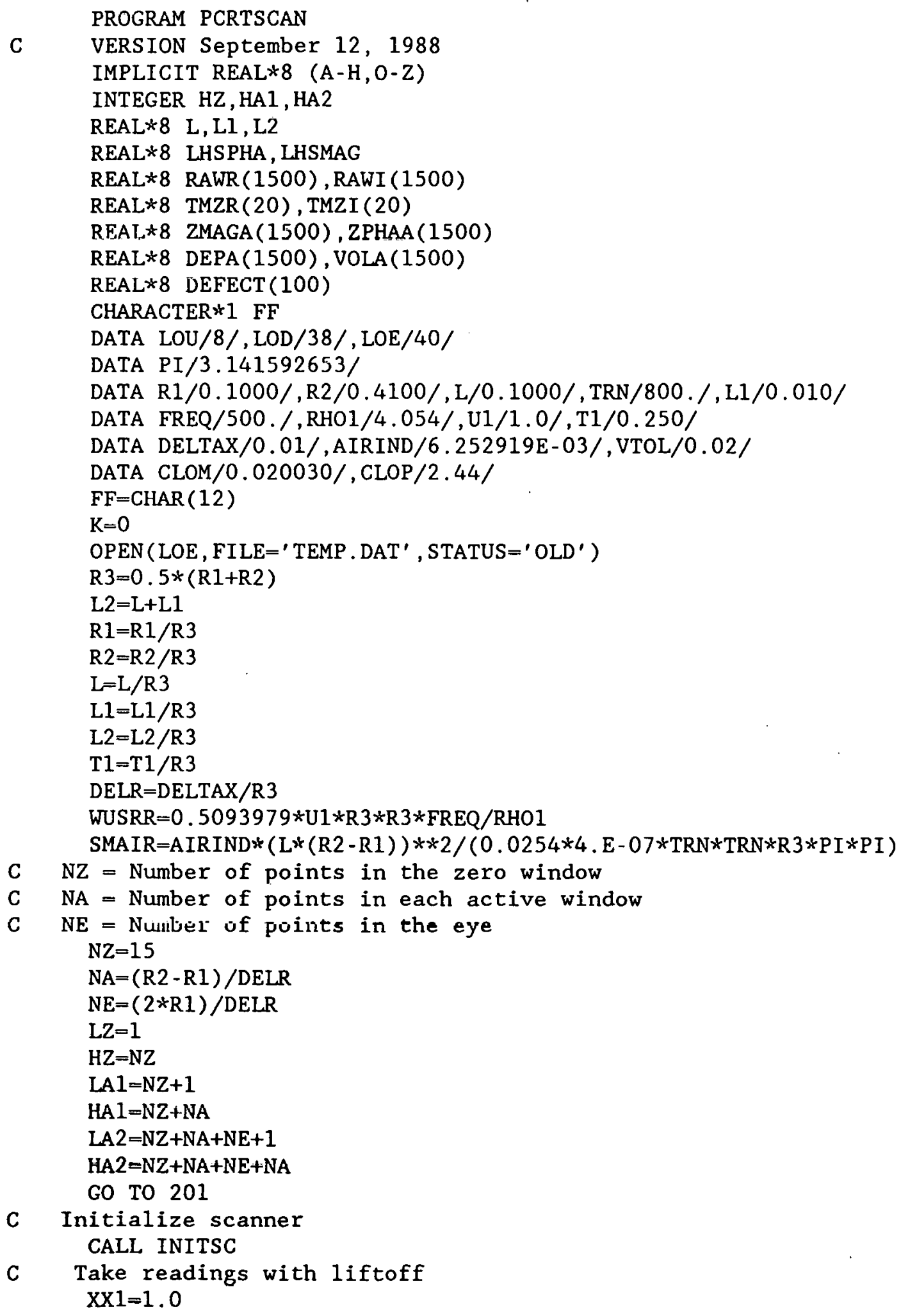




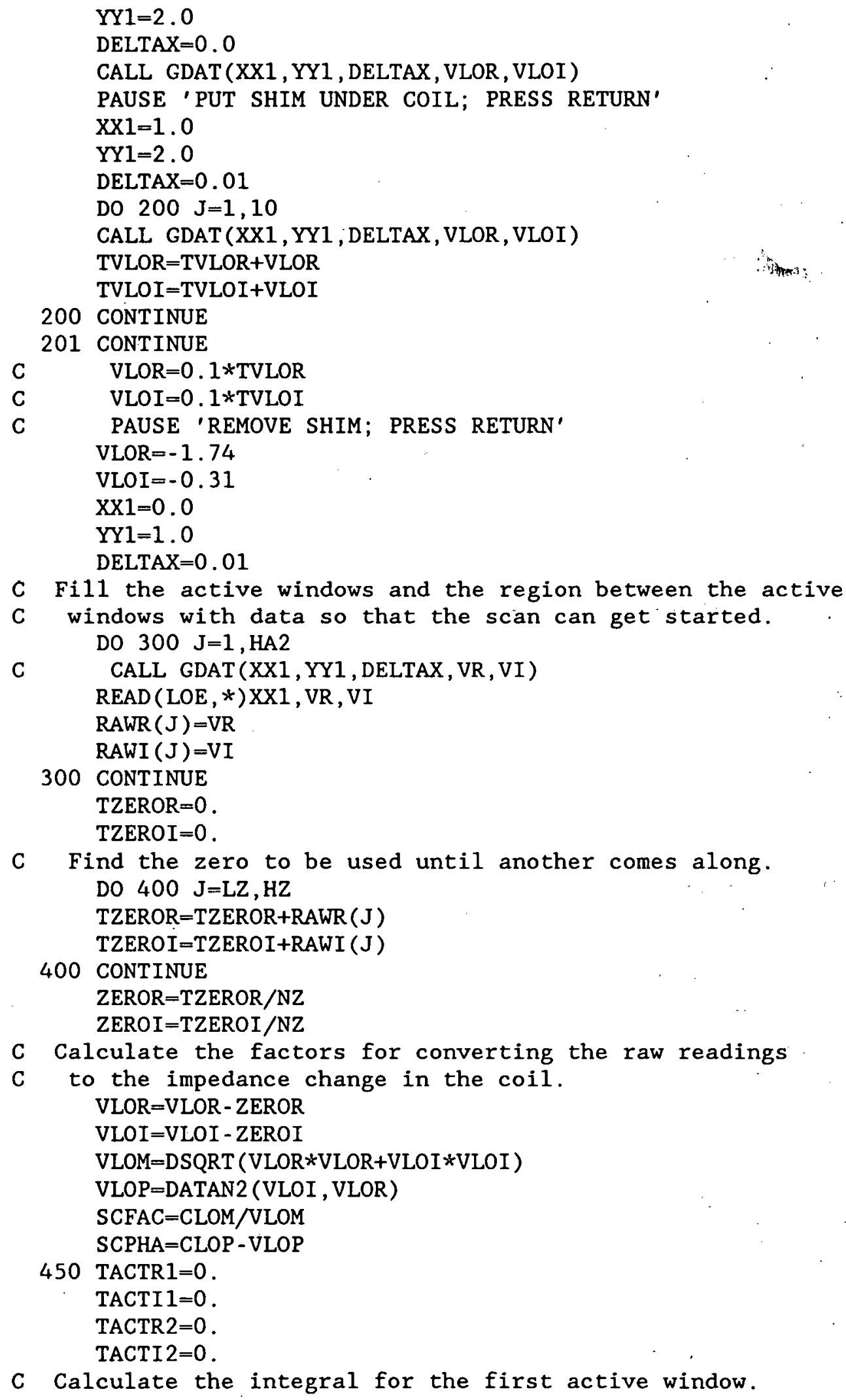

C Calculate the integral for the first active window. 


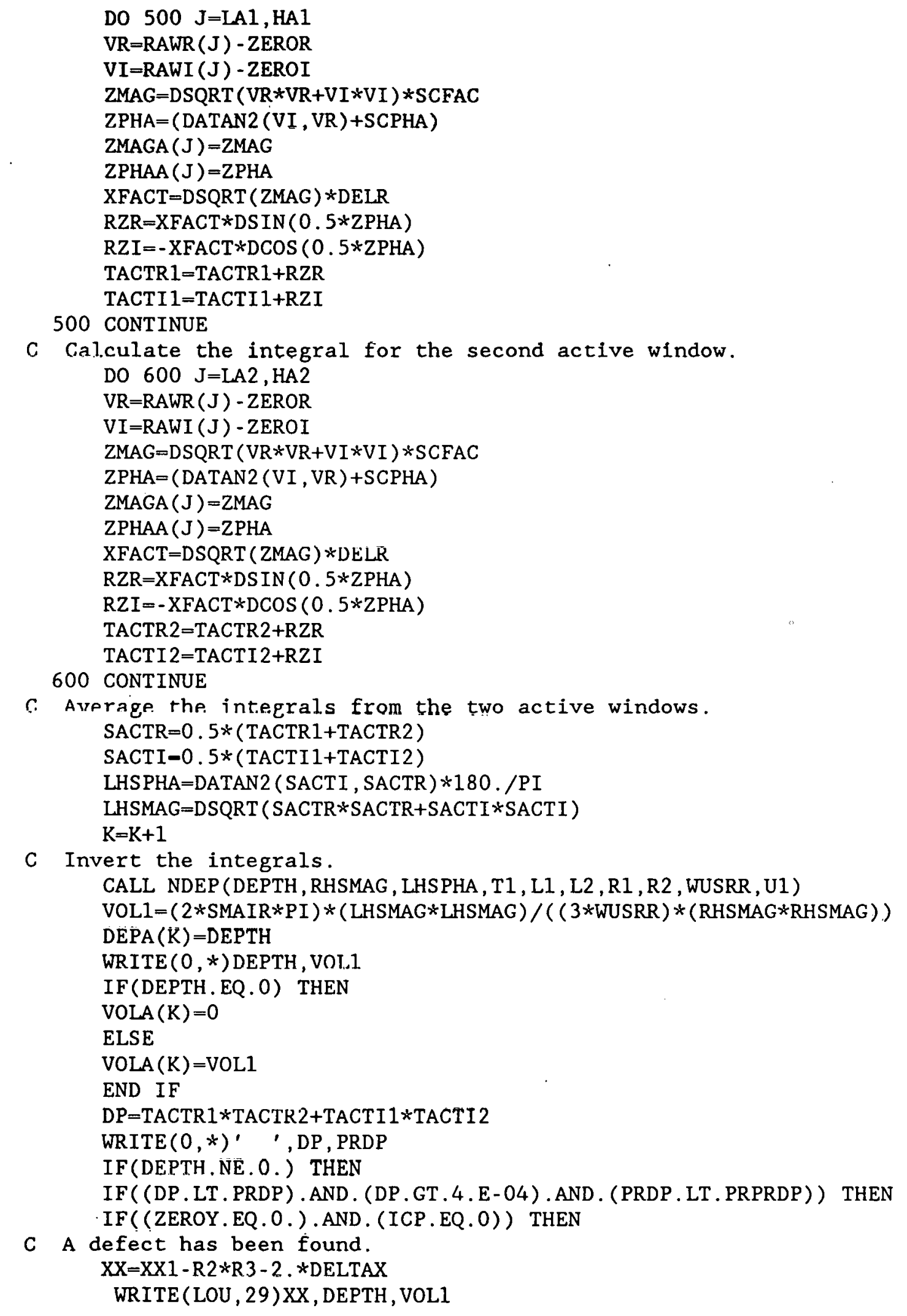




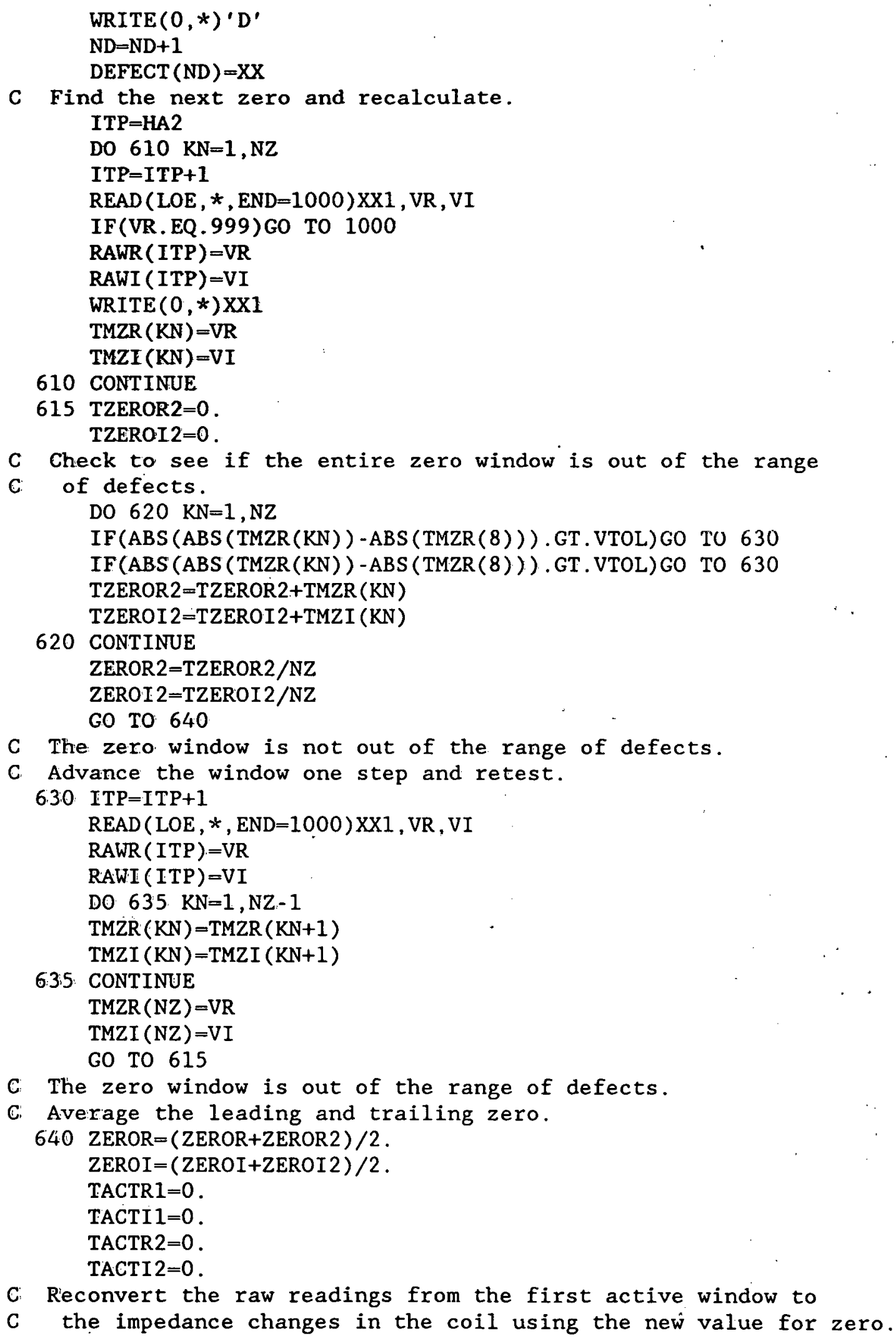




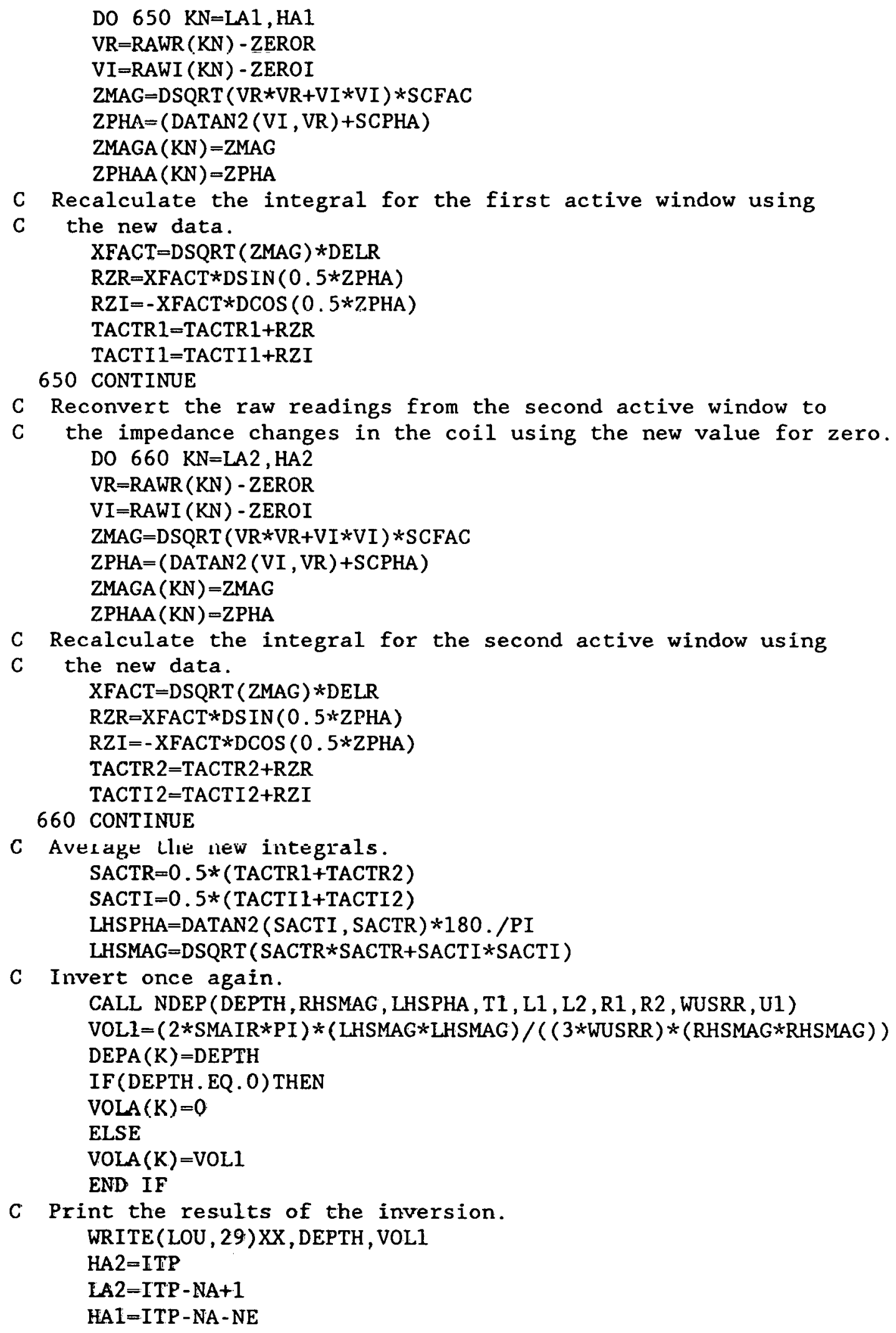




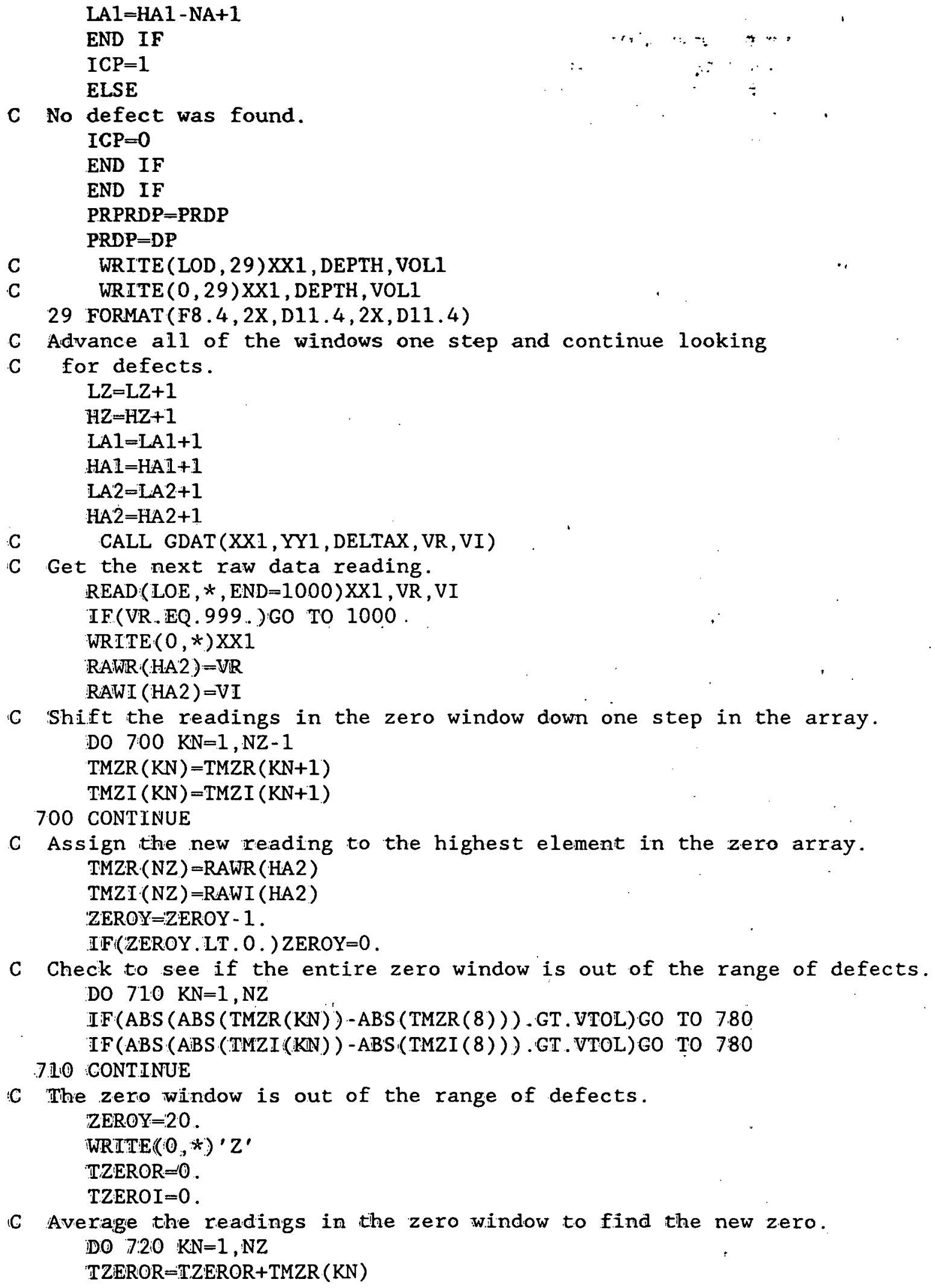

$C$ Check to see if the entire zero window is out of the range of defects. DO $710 \mathrm{KN}=1, \mathrm{NZ}$

$C$ The zero window is out of the range of defects.

ZEROY $=20$.

WRITE $(0, *)^{\prime} Z^{\prime}$

TZEROR $=0$.

TZEROI $=0$.

IC Average the readings in the zero window to find the new zero. DO $7: 20 \mathrm{KN}=1, \mathrm{NZ}$ TZEROR=TZEROR+TMZR (KN) 


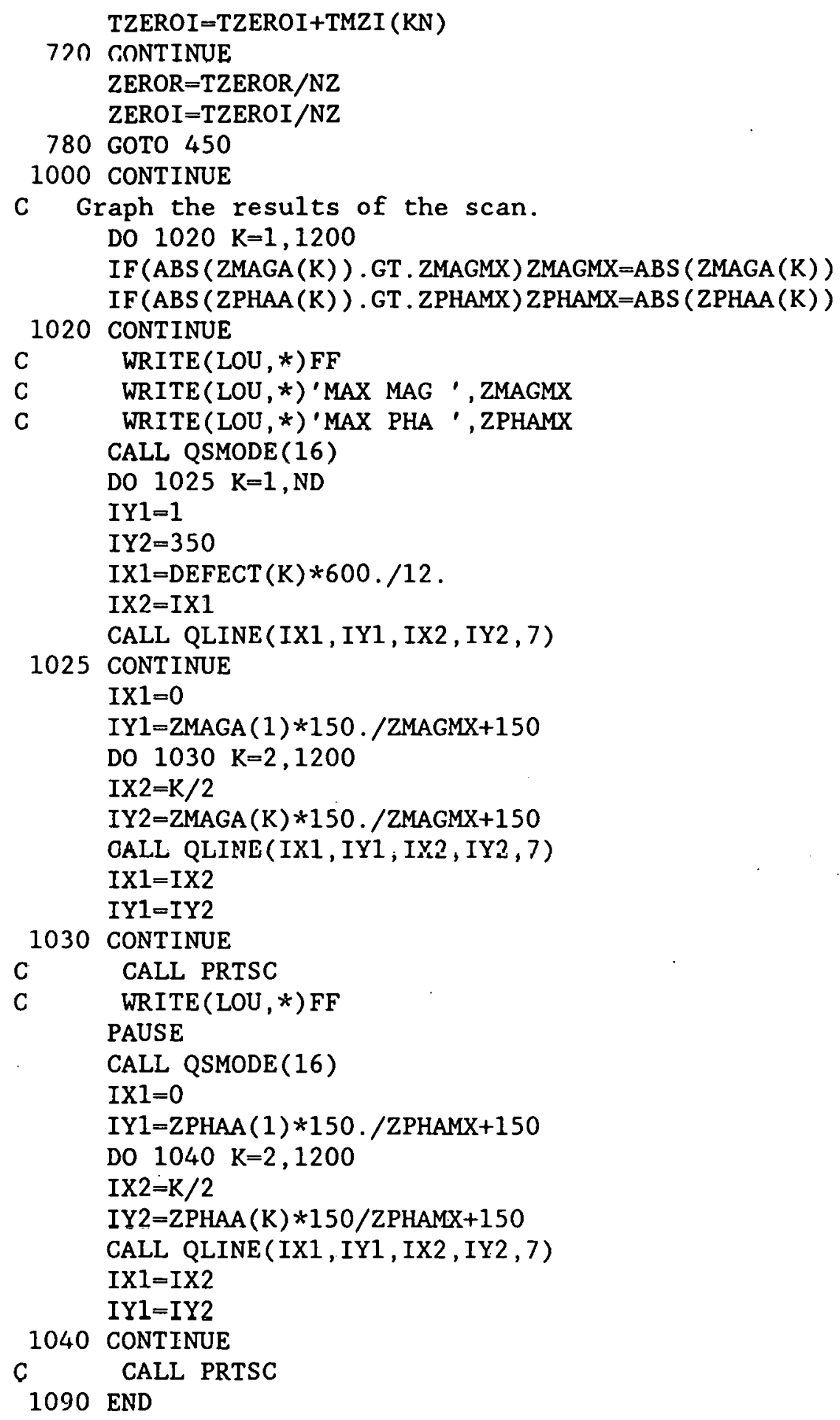


The programs in this section perform various functions relating to the effect of a defect in a single conducting plate on the induced voltage in the pickup coils of a reflection probe. A reflection probe above a conducting plate is shown in Fig. 8. The probe consists of a large driver coil with two pick-up coils mounted at either end. The pick-up coils are connected in opposition so that their signal cancels out with the probe in air. When the probe is placed on a conductor, the field can be considered to be equal to the field reflected back from the conductor.

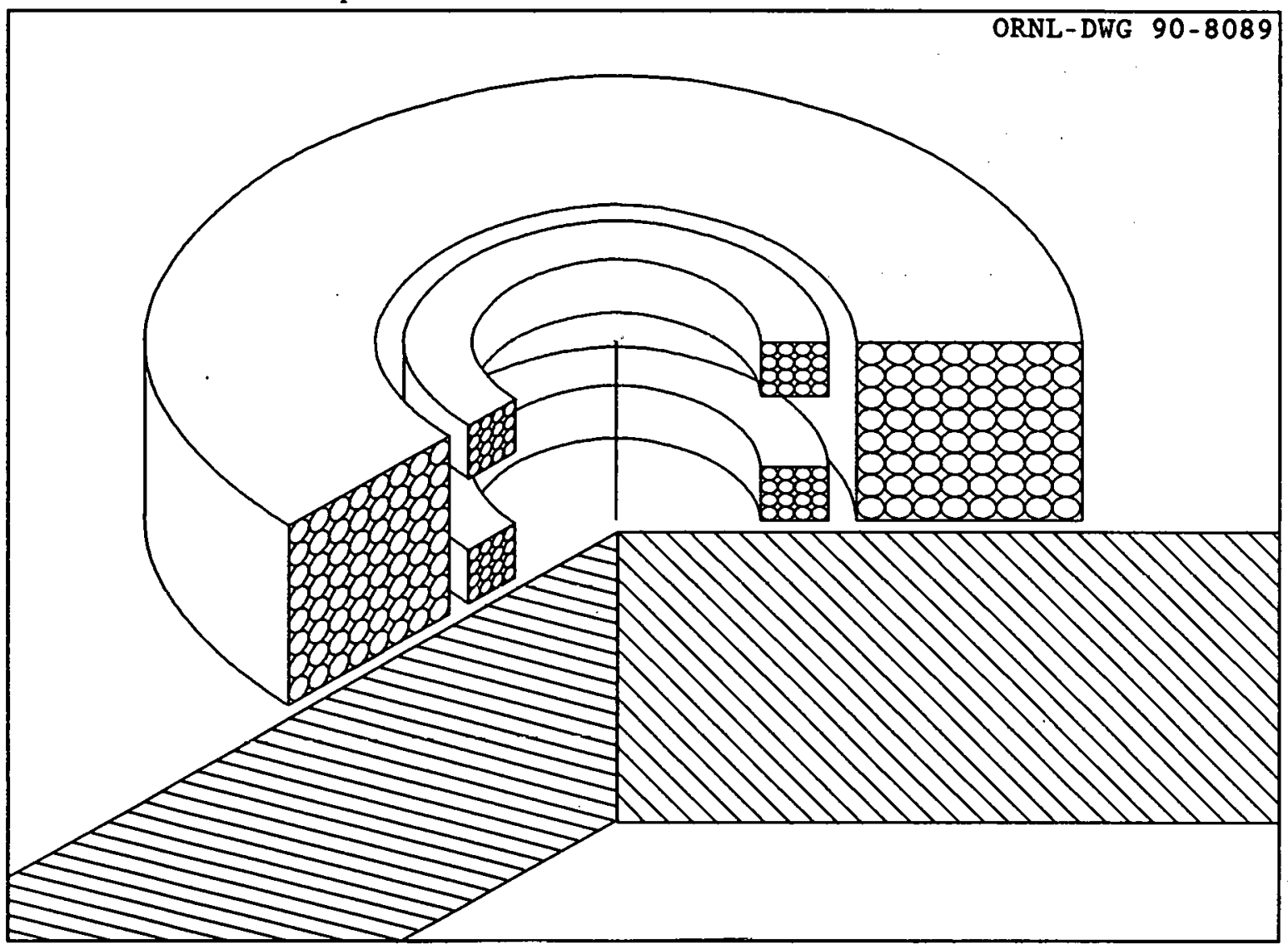

Fig. 8. Cutaway of a reflection probe above a conducting plate.

The electrical connections for the reflection coil circuit are shown in Figure 9. The voltage output from this circuit $V_{\text {out }}$ is given by:

$$
\begin{aligned}
V_{\text {out }}= & -j \omega M V_{0} R_{9} A \div\left\{\left(\omega C_{6} R_{0}-j\right)\left(\omega C_{7} R_{9}-j\right)(\omega M)^{2}+\right. \\
& {\left.\left[\left(\omega C_{6} R_{0}-j\right)\left(Z_{0}+R_{6}\right)-j R_{0}\right]\left[\left(\omega C_{7} R_{9}-j\right)\left(Z_{\mathrm{pu}}+R_{7}\right)-j R_{9}\right]\right\} }
\end{aligned}
$$

where $A$ is the amplifier gain and the rest of the terms are defined in Fig. 9 . 


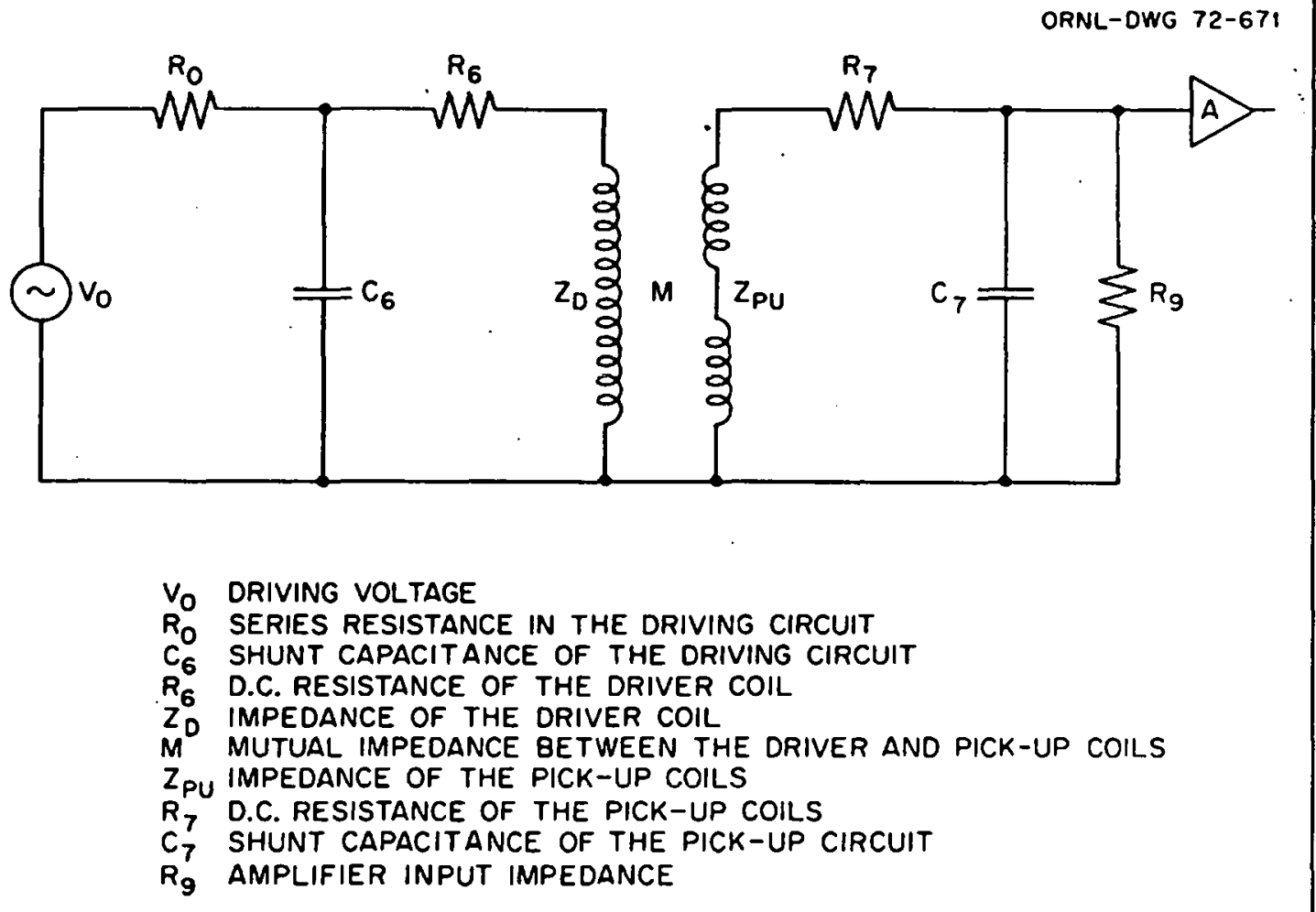

Fig. 9. Simplified circuit diagram for an eddy-current reflection type probe.

Fig. 10 shows a cross section of the reflection coil above the metal plate with the dimensions labeled as they are used in the equations and the programs. The programs are based on the signal from a small spherical defect, as shown in the figure.

As we can see from Eq. (20), we must calculate the impedance of both the driver and pick-up coils, as well as the mutual coupling between the two. The equations for the driver coil impedance, including the change due to the defect, is:

$$
\begin{gathered}
Z_{0}=\frac{\omega \pi \mu_{0} N_{3}^{2} R_{5}}{\left(r_{2}-r_{1}\right)^{2} \ell_{3}^{2}}\left\{j \int_{0}^{\infty} \frac{1}{\alpha^{6}} J^{2}\left(r_{2}, r_{1}\right)\left\{2\left(\alpha \ell_{3}+e^{-\alpha \ell_{3}}-1\right)+\left(1-e^{-\alpha \ell_{3}}\right)^{2} e^{-2 \alpha \ell_{6}} F\left(\alpha, \alpha_{1}, c\right)\right\} d \alpha\right. \\
\left.\frac{-V 0 I_{n} \alpha_{22} 3 \omega \mu \sigma \bar{r}^{2}}{8 \pi}\left[\int_{0}^{\infty} \frac{e^{-\alpha \ell_{6}}}{\alpha^{3}} J\left(r_{2}, r_{1}\right)\left(1-e^{-\alpha \ell_{3}}\right) J_{1}(\alpha r) F\left(\alpha, \alpha_{1}, z\right) d \alpha\right]^{2}\right\}
\end{gathered}
$$

where $F\left(\alpha, \alpha_{1}, z\right)=2 \alpha\left[\frac{\left(\alpha+\beta_{1}\right) \exp (\alpha, z)-\left(\alpha-\beta_{1}\right) \exp \left(-\alpha_{1}(2 c+z)\right)}{\left(\alpha+\beta_{1}\right)^{2}-\left(\alpha-\beta_{1}\right)^{2} \exp \left(-2 \alpha_{1} c\right)}\right]$ 


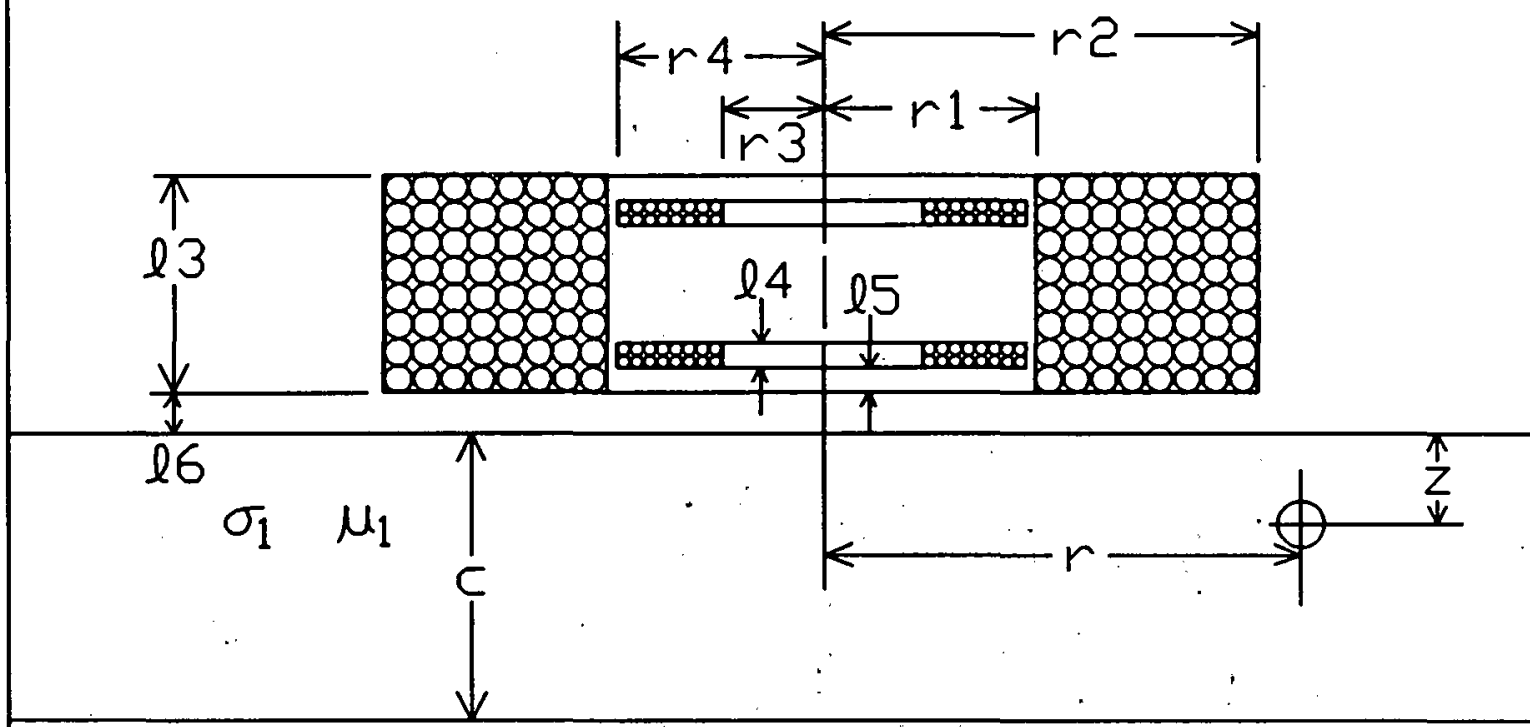

Fig. 10. Cross section of a reflection coil above a conducting plate with a spherical defect.

and $\quad F\left(\alpha, \alpha_{1}, c\right)=\left[\frac{\left(\alpha-\beta_{1}\right)\left(\alpha+\beta_{1}\right)-\left(\alpha-\beta_{1}\right)\left(\alpha+\beta_{1}\right) \exp (-2 \alpha, c)}{\left(\alpha+\beta_{1}\right)^{2}-\left(\alpha-\beta_{1}\right)^{2} \exp (-2 \alpha, c)}\right]$

and $\quad \beta_{1}=\left(\alpha^{2}+j \omega \mu \sigma_{1} \overline{\mathrm{r}}^{2}\right)^{1 / 2} / \mu$

The dimensions for the coil are shown in Fig. 10. For the pick-up coil Impedance we have: 


$$
\begin{aligned}
& Z_{\mathrm{p}}=\frac{\omega \pi \mu_{0} N_{4}^{2} R_{5}}{\left(r_{4}-r_{3}\right)^{2} \ell_{4}^{2}} \times\left\{j \int_{0}^{\infty} \frac{J^{2}\left(r_{4}, r_{3}\right)}{\alpha^{6}}\right. \\
& \left\{-4\left(\alpha \ell_{4}+e^{-\alpha \ell_{4}}-1\right)+\left(1-e^{-\alpha\left(l_{3}-\ell_{4}-2 l_{5}\right)}\right)^{2}\left(1-e^{-\alpha \ell_{4}}\right)^{-2} e^{-2 \alpha\left(l_{6}+l_{5}\right)} F\left(\alpha, \alpha_{1}, c\right) e^{-\alpha\left(l_{3}-2 l_{4}-2 l_{5}\right)}\right\} d \alpha \\
& \left.\frac{-V o 1_{n} \alpha_{22} 3 \omega \mu \sigma \bar{r}^{2}}{8 \pi}\left[\int_{0}^{\infty} \frac{e^{-\alpha\left(l_{6}+l_{5}\right)}}{\alpha^{3}} J\left(r_{4}, r_{3}\right)\left(1-e^{-\alpha\left(l_{3}-l_{4}-2 l_{5}\right)}\right)\left(1-e^{-\alpha l_{4}}\right) J_{1}(\alpha r) F\left(\alpha, \alpha_{1}, z\right) d \alpha\right]^{2}\right\}
\end{aligned}
$$

and for the mutual impedance between the driver and pickup coils we have:

$$
\begin{aligned}
& j \omega M=\frac{\omega \pi \mu_{0} N_{3} N_{4} R_{5}}{\left(r_{2}-r_{1}\right) \ell_{3}\left(r_{4}-r_{3}\right) \ell_{4}} \times \\
& \left\{j \int_{0}^{\infty} \frac{J\left(r_{2}, r_{1}\right) J\left(r_{4}, r_{3}\right)}{\alpha^{6}}\left(1-e^{-\alpha \ell_{3}}\right)\left(1-e^{-\alpha\left(l_{3}-\ell_{4}-2 l_{5}\right)}\right)\left(1-e^{-\alpha \ell_{4}}\right) e^{-\alpha\left(2 l_{6}+\ell_{5}\right)} F\left(\alpha, \alpha_{1}, c\right) d \alpha .\right. \\
& \frac{-V o 1_{n} \alpha_{22} 3 \omega \mu \sigma \bar{r}^{2}}{8 \pi}\left[\int_{\theta}^{\infty} \frac{e^{-\alpha \ell_{6}}}{\alpha^{3}} J\left(r_{2}, r_{1}\right)\left(1-e^{-\alpha \ell_{3}}\right) J_{1}(\alpha r) F\left(\alpha, \alpha_{1}, z\right) d \alpha\right] \times
\end{aligned}
$$

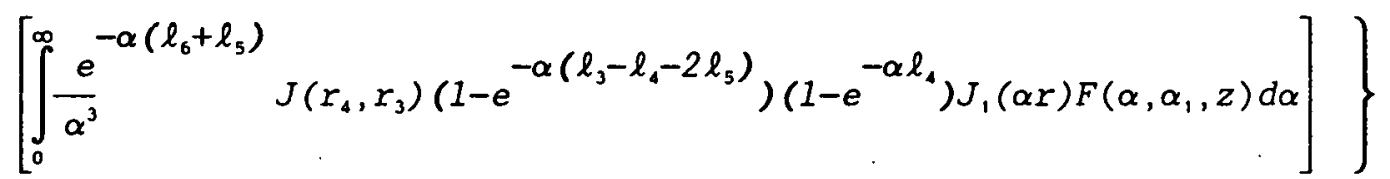

These are the basic programs used for the calculation of the change in magnitude and phase of the eddy-current signal due to a defect for a reflection probe. In Eqs. (21), (25), and (26) the terms multiplied by $j$ are the normal values without the defect, while the terms multiplied by $V o I_{n}$ are the changes due to the defect. If the values of driving and input impedance, $R_{0}$ and $R_{9}$, are large and the circuit is operated well below resonance, the major change due to a defect will be in the term for $M$. Most of the tests run are usually designed for these conditions.

The program RFDSF calculates the defect sensitivity factor of a reflection coil at points throughout a conducting plate, and program RFDSFPLT produces a contour plot of these calculations. Program RFAVZSCN does the theoretical calculations for the change in the induced voltage in a pickup coil due to a defect in a conducting plate, and program RFFIX converts raw experimental data to the change in induced voltage in the coil. Program RFGRAPH can plot the data from these two programs side-by-side so the theoretical predictions for a defect can be compared to the actual data. Finally, programs RFBLDF and RFINV can take data stored by either RFAVZSCN 
or RFFIX and solve for the depth and volume of the defect that produced that data. These programs and their discussion closely parallel the programs for the pancake coils in the previous section. 
RFBLDF builds a lookup file of magnitude and phase of DSF

Program RFBLDF builds a lookup file containing the magnitude and phase of the integral of the defect sensitivity factor of a reflection coil with respect to $r$, the radial distance from the coil axis to the point where the calculations are performed, from the inner radius of the pickup coil to the outer radius of the driver coil at different depths in a plate. This lookup file can be used by program RFINV to calculate the depth and volume of defects. (See note \#2.)

\section{Summary}

1. Dimension arrays and declare variable types.

2. Initialize variables.

3. Select a value for the depth in the plate at which the calculations are to be performed.

4. Select a value for the radial distance between the coil axis and the point at which the calculations are to be performed.

5. Find the defect sensitivity factor at this point.

6 . Loop to 4 until done.

7. Calculate the integral of the defect sensitivity factor over the range of $r$ and write the result to a data file.

8. Loop to 3 until done.

\section{Variables}

NOTE: Most of the variables which occur inside the integration loops are uninteresting because they do not correspond to anything physical and because they should never require user intervention. Therefore, these variables will not be discussed here. For a detailed description of these variables, see the documentation of program RFAVZSCN. For a discussion of the major differences between the integration section of program RFBLDF and the integration section of program. RFAVZSCN, see note \#1.

Starred variables must be set by the user.

$66^{\circ} \quad$ The total shunt capacitance in farads of the

C7. The total shunt capacitance in farads of the pickup circult.

DELTAR The normalized radial distance between successive data points.

FREQ The operating frequency in hertz.

GAIN $\quad$ Gain of pickup amplifier.

L3 The normalized length of the driver coil.

L4 The normalized length of the pickup coil.

L5 The normalized distance of recess of the pickup coil.

L6 The normalized lift-off of the driver coil.

LOD The number of the I/O unit connected to the output 
LOU

MZT'

NPROBE ${ }^{*}$

NRT

NS

NZT

$\mathrm{RO}^{\circ}$

R1

$\mathrm{R} 2$

R3

R4

R5

R6

R7

R9 ${ }^{\circ}$

RD

RHO1*

RHSI

RHSM

RHSP

RHSR

data file.

The number of the $I / O$ unit connected to the printer.

The number of depths throughout the plate at which the program does calculations. Increasing this number usually improves the accuracy of the inversion somewhat because it brings the interpolated points closer together, so the variation of the magnitude and phase of the integral between the points is more nearly linear. (See note \#4.)

Character variable which contains the name of the reflection probe to be used in the calculations. The total number of points in the radial direction at which calculations are performed. (See note \#4.)

The side where the defect is located. If $N S=1$, the defect is on the near side; if $N S=2$, the defect is on the far side. The value of NS is assigned according to the location of the center of the defect. If the center of the defect is nearer the near side of the plate, NS is set equal to 1. If the center of the defect is nearer the far side of the plate, NS is set equal to 2 .

The number of parts each defect is divided into along its axis to perform the calculations. (See note \#4.)

Output series resistance of driving amplifier in ohms.

The normalized inner radius of the driver coil.

The normalized outer radius of the driver coil.

The normalized inner radius of the pickup coil.

l'he normalized outer radius of the pickup coil.

The mean radius of the driver coil in inches.

DC resistance of the driver coil in ohms.

DC resistance of both pickup coils in ohms.

Input shunt resistance of pickup amplifier in ohms.

The radial distance from the axis of the coil to the point where the calculations are being done.

The resistivity of the plate in $\mu \Omega-\mathrm{cm}$.

The imaginary part of the integral of the defect sensitivity factor with respect to $R D$ from $R 3$ to R2.

The magnitude of the integral of the defect sensitivity factor with respect to $R D$ from $R 3$ to R2.

The phase in radians of the integral of the defect sensitivity factor with respect to $R D$ from $R 3$ to R2.

The real part of the integral of the defect 
sensitivity factor with respect to $R D$ from $R 3$ to

R2.

T1 $\quad$ The thickness of the plate. It is input in inches and normalized by the program.

TD The density of turns in the driver coil.

TNDR The number of turns in the driver coil.

TNPU The number of turns in each pickup coil.

TP The density of turns in the pickup coil.

$\mathrm{U} 1^{\circ}$ The relative magnetic permeability of the plate. VIN W Output voltage of driving amplifier in volts.

The angular operating frequency.

The normalized distance from the side of the plate where the defect is located to the bottom of the defect. A negative number.

2D2

The normalized distance from the near side of the plate to the center of the defect. A negative number.

ZDTIO The imaginary part of the self impedance of the driver coil with no defects present.

ZDTRO The real part of the self impedance of the driver coil with no defects present.

ZMSTEP The normalized axial distance between the depths at which the program does the calculations.

ZMTDI The imaginary part of the change in the mutual impedance between the driver coil and the pickup coil due to a defect.

ZMTDR The real part of the change in the mutual impedance between the driver coil and the pickup coil due to a defect.

ZMTI0 The imaginary part of the mutual impedance between the driver coil and the pickup coil with no defects present.

ZMTR0 The real part of the mutual impedance between the driver and pickup coils with no defects present.

ZPTIO The imaginary part of the self impedance of the pickup coil with no defects present.

ZPTRO The real part of the self impedance of the pickup coil with no defects present.

\section{Notes}

1. The integration in this program is very similar to the integration in program RFAVZSCN, but some very minor differences do exist. Most of these come from the fact that this program calculates only the defect sensitivity factor, which does not depend on the volume of the defect, and program RFAVZSCN calculates the voltage change due to the defect, which does depend on the volume of the defect. While it is necessary to calculate the defect sensitivity factor to calculate the voltage change due to the defect, program RFAVZSCN never calculates the defect 
sensitivity factor as a separate quantity. Thus, some of the variables in RFAVZSCN which have the same name as variables in program RFBLDF have the defect volume as an extra factor.

2. The way that program RFINV uses the output of this program to calculate the depth and volume of a defect is very simple. Program RFINV takes either experimental data stored by program RFFIX or calculated data stored by program RFAVZSCN to find the integral of the voltage change due to the defect with respect to $r$ from the inner radius of the pickup coil to the outer radius of the driver coil. The phase of this integral depends only upon the depth of the defect, and it is a single valued function of the depth of the defect. (See note \#3.) The phase of the integral of the defect sensitivity factor will be the same as the phase of the voltage change due to the defect, because the defect sensitivity factor differs from the voltage change only by a constant real factor. Therefore, program RFINV can search through the file created by program RFBLDF until it finds the depth corresponding to the phase it obtained when it calculated the integral. This will be the depth of the defect. Then program RFINV can divide the integral it calculated by the integral calculated by program RFBLDF. Since the phases of the integrals are equal, the factors which contain the phase will cancel, and the program needs only to divide the magnitude of one integral by the other. The result of this division is the constant factor by which the two integrals differ, which is equal to the defect volume multiplied by $\alpha_{22}$, the defect shape and orientation factor. We normally assume that $\alpha_{22}$ is equal to 1 , so the program is left with the defect volume.

3. One of the necessary assumptions for the reflection coil inversion programs to work is that the phase of the integral calculated by this program depends only upon the depth of the defect and that it is therefore independent of the defect volume. This is not exactly true. The expression for the defect sensitivity factor contains terms in the denominator which do depend slightly on the defect volume, and since this volume dependence cannot be factored out, it has a small effect on the phase of the integral. However, the error introduced by this slight volume dependence is not significant, as shown by the accuracy of the inversion of data calculated by program RFAVZSCN, which considers the dependence of the phase on the volume. The error for these inversions is typically less than one percent.

4. The selection of the point in the plate about which the calculations are done is complex in the program. The outside position determining loop runs from 0 to MZT. This loop sets the value of the depth of the center of the defect by assigning values to variables $Z D$ and $Z D 2$. It also determines whether a defect is on the near side of the plate or on the far side of the plate. The next position determining loop, which occurs inside this one, runs from 1 to NRT. It sets the value for the radial distance from the coil axis to the center of the defect by assigning a value to variable $\mathrm{RD}$. The final position determining loop lies inside both of these, and it runs from 1 to NZT. This loop is present for the purpose of dividing the large defect located at cylindrical coordinates 
RD and ZD2 into a number of smaller defects so that more accurate calculations can be obtained for the entire defect. In the case of a near side defect, the large defect is divided into NZT smaller defects centered at even intervals along the axis of the large defect between the bottom of the defect and the near side of the plate. In the case of a far side defect, the large defect is divided into. NZT smaller defects centered at even intervals along the axis of the large: defect between the bottom of the defect and the far side of the pliate.. 


\section{Listing}

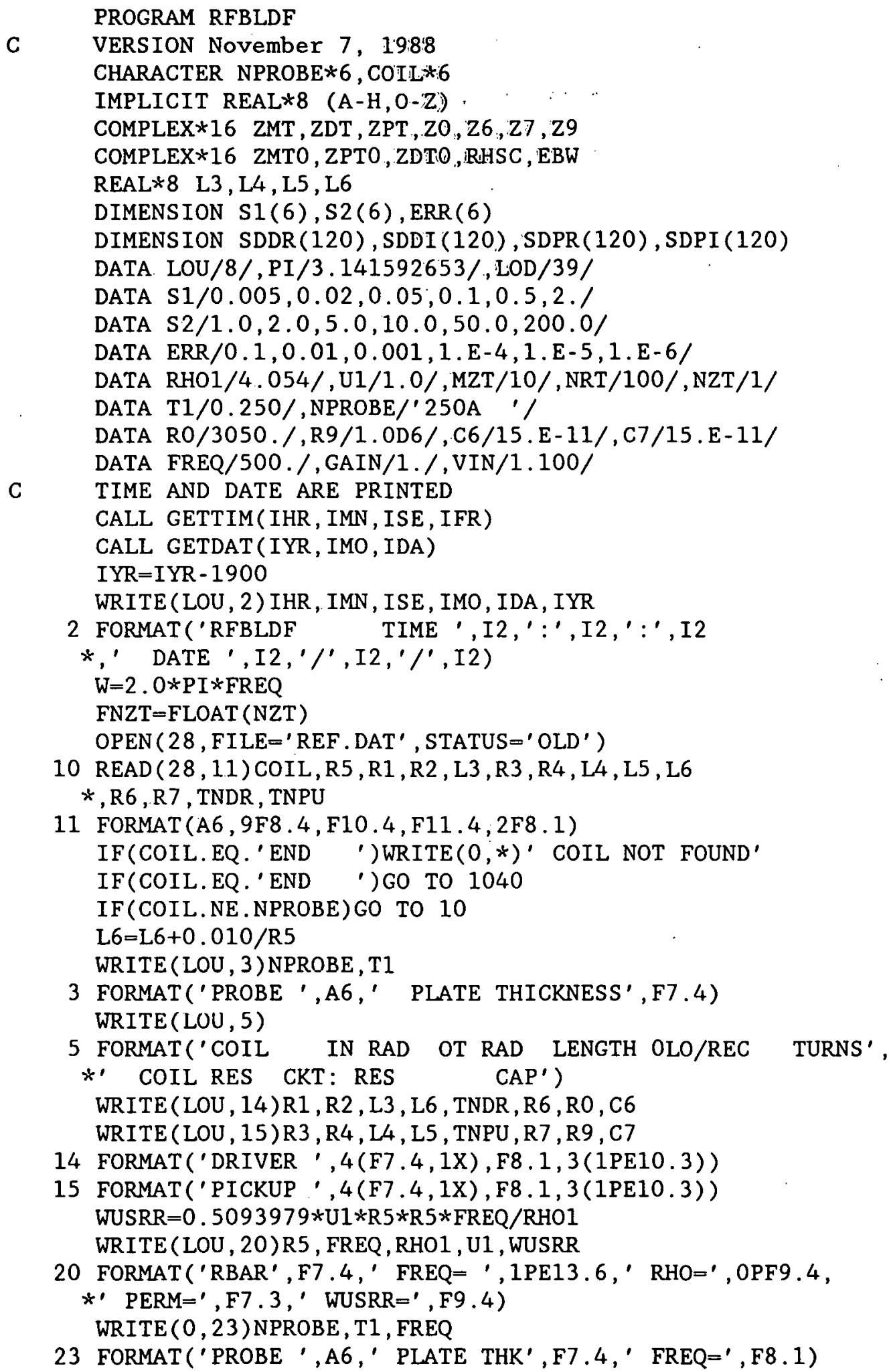


WRITE (LOU, *)

WRITE (LOU, 24)

24 FORMAT ('DEPTH MAGNITUDE PHASE')

$\mathrm{T} 1=\mathrm{T} 1 / \mathrm{R} 5$

ZMSTEP $=\mathrm{T} 1 / \mathrm{MZT}$

DELTAR $=($ R2 - R3 $) /$ NRT

$\mathrm{TD}=\mathrm{TNDR} /((\mathrm{R} 2-\mathrm{R} 1) * \mathrm{~L} 3)$

$\mathrm{TP}=\mathrm{TNPU} /((\mathrm{R} 4-\mathrm{R} 3) * \mathrm{~L} 4)$

$\mathrm{ZDF}=6.300475 \mathrm{E}-7 * \mathrm{FREQ} * \mathrm{TD} * \mathrm{TD} * \mathrm{R} 5$

$\mathrm{ZPF}=6.300475 \mathrm{E}-7 * \mathrm{FREQ} * \mathrm{TP} * \mathrm{TP} * \mathrm{R} 5$

$\mathrm{ZMF}=6.300475 \mathrm{E}-7 * \mathrm{FREQ} * \mathrm{TD} * \mathrm{TP} * \mathrm{R} 5$.

$D M F=0.1193662 *$ WUSRR

DO $1020 \mathrm{MZ}=0, \mathrm{MZT}$

WRITE $(0, *)^{\prime} M Z$ ',MZ

IF (MZ . GT . (0.5*MZT)) THEN

C Far side defect

$\mathrm{NS}=2$

$\mathrm{ZM}=(\mathrm{MZT}-\mathrm{MZ}) * \mathrm{ZMSTEP}$

$\mathrm{ZD}=-2 . * \mathrm{ZM}$

$\mathrm{ZM}=\mathrm{MZ} * \mathrm{ZMSTEP}$

ZD2 $=-Z M$

ELSE

C Near side defect

$\mathrm{NS}=1$

$\mathrm{ZM}=\mathrm{MZ} * \mathrm{ZMSTEP}$

$Z D=-2 . * Z M$

$\mathrm{ZD} 2=-\mathrm{ZM}$

END IF

RD-R3 (0.5\%DELTAR)

SRHSR $=0$.

SRHS I $=0$.

DO $1010 \mathrm{NR}=1$, NRT

$\operatorname{WRITE}(0, *) M Z, N R$

$R D=R D+D E L T A R$

SAIR $1=0.0$

SAIR2 $=0.0$

SZDR $=0.0$

SZDI $=0.0$

$S Z P R=0.0$

SZPI $=0.0$

$\mathrm{SZMR}=0.0$

SZMI $=0.0$

DO $25 \mathrm{NZ}=1$, NZT

$\operatorname{SDDR}(\mathrm{NZ})=0.0$

$\operatorname{SDDI}(N Z)=0.0$

$\operatorname{SDPR}(\mathrm{NZ})=0.0$

$\operatorname{SDPI}(\mathrm{NZ})=0.0$

25 CONTINUE

$\mathrm{B} 1=0.0$

$\mathrm{B} 2=\mathrm{S} 2$ (1)

DO $100 \mathrm{JKL}=1,6$ 
30 RI9=SAIR1

$\mathrm{X}=\mathrm{B} 1-0.5 * \mathrm{~S} 1$ (JKL)

C

DETERMINE NUMBER OF STEPS, ISTEPS, BASED ON STEP SIZE\&INTERVAL

ISTEPS $=\operatorname{DNINT}((\mathrm{B} 2-\mathrm{B} 1) / \mathrm{S} 1(\mathrm{JKL}))$

DO $90 \quad I=1$, ISTEPS

$\mathrm{X}=\mathrm{X}+\mathrm{S} 1(\mathrm{JKL})$

CALL BESSEL(XJR2, $X, R 2)$

CALL BESSEL(XJR1,X,R1)

CALL BESSEL(XJR4, $X, R 4$ )

CALL BESSEL(XJR3,X,R3)

R21 $=X J R 2-X J R 1$

$R 43=X J R 4-X J R 3$

$\mathrm{XL} 3=\mathrm{X} * \mathrm{~L} 3$

IF (XL3.GT.5.0E - 3) GO TO 60

$\mathrm{A} 1=\mathrm{XL} 3 * \mathrm{XL} 3 *(0.5-\mathrm{XL} 3 / 6.0)$

GO TO 80

60 IF (XL3.GT.75.0) GO TO 70

$\mathrm{Al}=\mathrm{XL} 3+\mathrm{DEXP}(-\mathrm{XL} 3)-1.0$

GO TO 80

$70 \mathrm{Al}=\mathrm{XL} 3-1.0$

$80 \quad A 3=X L 3-A 1$

$\mathrm{SFD}=\mathrm{S} 1(\mathrm{JKL}) * \mathrm{R} 21 * \mathrm{R} 21$

$\mathrm{SFP}=\mathrm{S} 1(\mathrm{JKL}) * \mathrm{R} 43 * \mathrm{R} 43$

$\mathrm{SFM}=\mathrm{S} 1(\mathrm{JKL}) * \mathrm{R} 21 * \mathrm{R} 43$

$S A I R 1=S A I R 1+S F D * 2.0 * A 1$

$\mathrm{XI} 4=\mathrm{X} * \mathrm{~L} 4$

IF (XL4..GT.5.OE - 3) GO TO 81

$\mathrm{A} 2=\mathrm{XL} 4 \times \mathrm{XL} 4 *(0.5-\mathrm{XL} 4 / 6.0)$

GO TO 83

81 IF(XI4.GT.75.0) GO TO 82

$\mathrm{A} 2=\mathrm{XL} 4+\mathrm{DEXP}(-\mathrm{XL} 4)-1.0$

GO TO 83

$82 \mathrm{~A} 2=\mathrm{XL} 4-1.0$

83 A4 $=X L 4-A 2$

$A 13=1.0-A 3$

A14 $=1.0-A 4$

$\mathrm{A} 5=\mathrm{DEXP}(-\mathrm{X} * \mathrm{~L} 5)$

$\mathrm{XL} 8=\mathrm{X} *(\mathrm{~L} 3-2 . * \mathrm{~L} 4-2 . * \mathrm{~L} 5)$

IF (XL8.GT . 75.) A8 $=0$.

IF (XL8 . LE . 75.) A8 $=\operatorname{DEXP}(-\mathrm{XL8})$

SAIR2 $=S A I R 2+S F P *(4.0 * A 2-2.0 * A 4 * A 4 * A 8)$

IF $(X *$ L6.GT. 75. $)$ GO TO 90

C

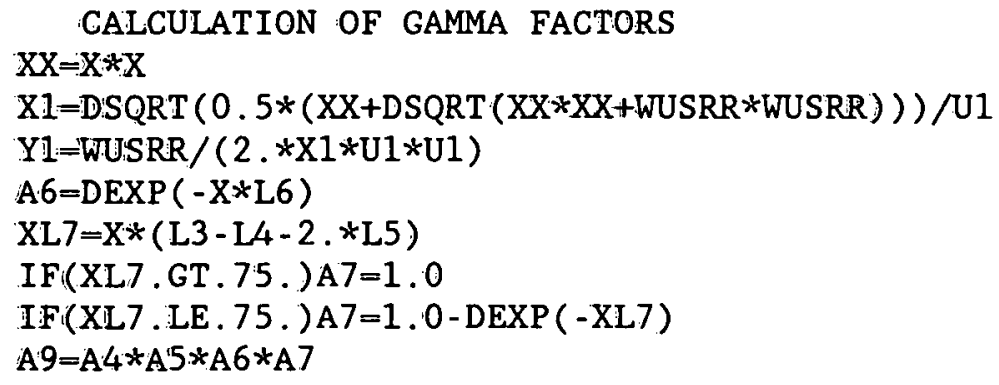




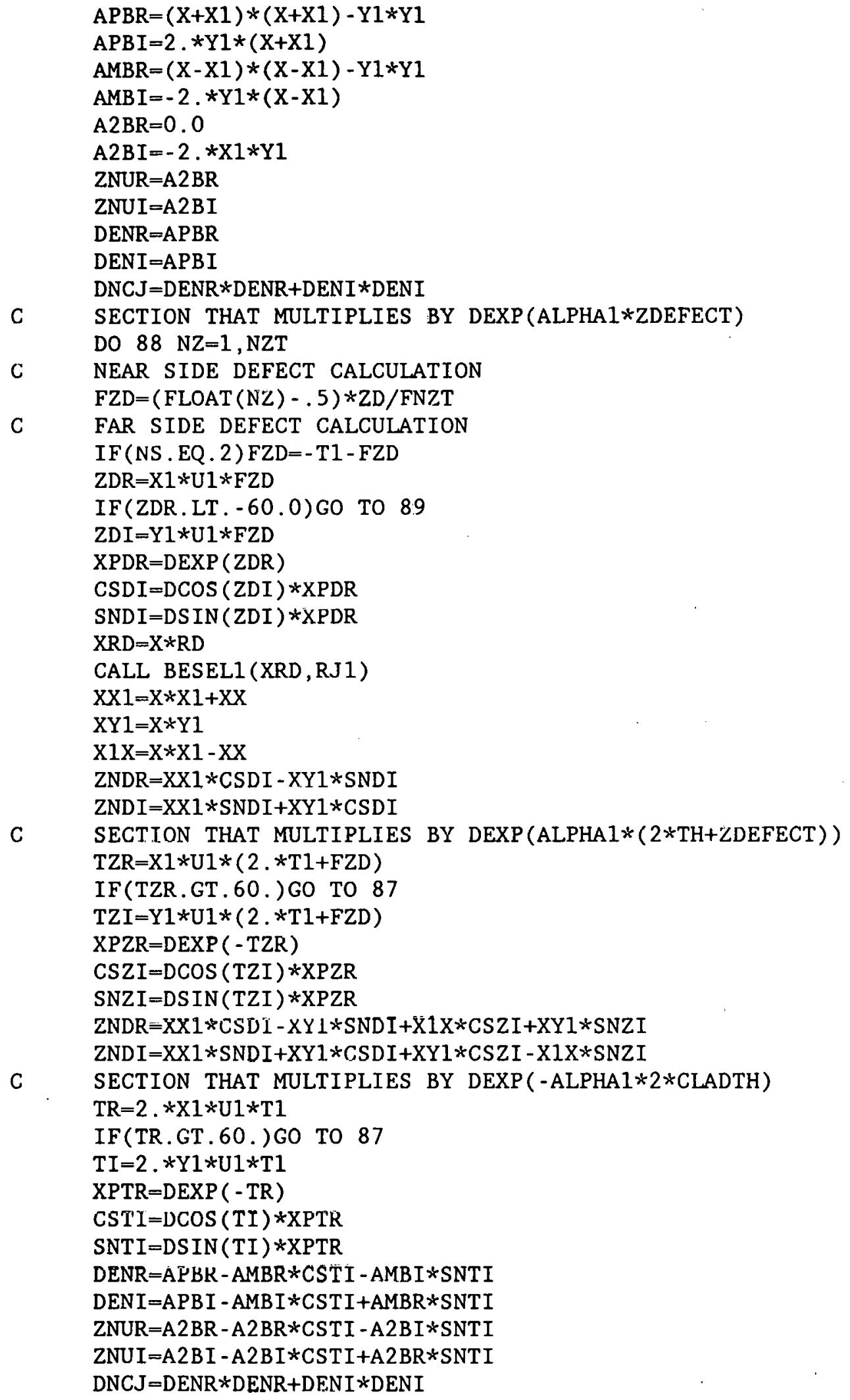


$87 \mathrm{ZDRL}=(\mathrm{ZNDR} * \mathrm{DENR}+\mathrm{ZNDI} * \mathrm{DENI}) / \mathrm{DNCJ}$ $Z D I M=(D E N R * Z N D I-Z N D R * D E N I) / D N C J$

C SUM DEFECT SENSITIVITY FACTORS FOR THE DRIVER \& PICKUP COILS $\operatorname{SDDR}(\mathrm{NZ})=\operatorname{SDDR}(\mathrm{NZ})+\mathrm{A} 3 * \mathrm{~A} 6 * \mathrm{RJ} 1 * 2 * \mathrm{ZDRL} * \mathrm{R} 21 * \mathrm{~S} 1(\mathrm{JKL})$

$\operatorname{SDDI}(\mathrm{NZ})=\operatorname{SDDI}(\mathrm{NZ})+\mathrm{A} 3 * \mathrm{~A} 6 * \mathrm{RJ} 1 * 2 * \mathrm{ZDIM} * \mathrm{R} 21 * \mathrm{~S} 1(\mathrm{JKL})$

$\operatorname{SDPR}(\mathrm{NZ})=\operatorname{SDPR}(\mathrm{NZ})+\mathrm{A} 9 * \mathrm{RJ} 1 * 2 * \mathrm{ZDRL} * \mathrm{R} 43 * \mathrm{~S} 1(\mathrm{JKL})$ $\operatorname{SDPI}(\mathrm{NZ})=\operatorname{SDPI}(\mathrm{NZ})+\mathrm{A} 9 * \mathrm{RJ} 1 * 2 * \mathrm{ZDIM} * \mathrm{R} 43 * \mathrm{~S} 1(\mathrm{JKL})$

88 CONTINUE

$89 \mathrm{ZRL}=(\mathrm{ZNUR} * \mathrm{DENR}+\mathrm{ZNUI} * \mathrm{DENI}) / \mathrm{DNCJ}$

$\mathrm{ZIM}=(\mathrm{DENR} * \mathrm{ZNUI}-\mathrm{ZNUR} * \mathrm{DENI}) / \mathrm{DNCJ}$

$S Z D I=S Z D I+A 3 * A 3 * A 6 * A 6 * Z R L * S F D$

$S Z D R=S Z D R-A 3 * A 3 * A 6 * A 6 * Z I M * S F D$

$S Z P I=S Z P I+A 9 * A 9 * Z R L * S F P$

$S Z P R=S Z P R-A 9 * A 9 * Z I M * S F P$

$S Z M I=S Z M I+A 3 * A 6 * A 9 * Z R L * S F M$

$S Z M R=S Z M R-A 3 * A 6 * A 9 * Z I M * S F M$

90 CONTINUE

$\mathrm{B} 1=\mathrm{B} 2$

$\mathrm{B} 2=\mathrm{B} 2+\mathrm{S} 2$ (JKL)

CHECK $=($ SAIR $1-$ RI9) $/$ SAIR1

IF (ABS (CHECK) . GT.ERR (JKL)) GO TO 30

100 CONTINUE

C

COMPUTATION OF DRIVER INDUCTANCE

$\mathrm{Q} 6=\mathrm{ZDF} * \mathrm{SAIR} 1 / \mathrm{W}$

C DEFINE COMPLEX QUANTITIES THAT ARE CONSTANT

$Z 0=\operatorname{DCMPLX}(0.0 \mathrm{DO},-\mathrm{RO})$

$\mathrm{Z} 6=\mathrm{DCMPLX}(\mathrm{W} * \mathrm{C} 6 * \mathrm{R} 0,-1.0 \mathrm{D} 0)$

$\mathrm{Z7}=\mathrm{DCMPLX}(\mathrm{W} * \mathrm{C} 7 * \mathrm{R} 9,-1.0 \mathrm{DO})$

$Z .9=D C M P L X(0.0 D 0,-R 9)$

C AVERAGE DEFECT VALUES OVER DEPTH

$A D D R=0.0$

$\mathrm{ADDI}=0.0$

$\mathrm{ADPR}=0.0$

$A D P I=0.0$

DO $125 \mathrm{NZ}=1, \mathrm{NZT}$

$\mathrm{ADDR}=\mathrm{ADDR}+\mathrm{SDDR}(\mathrm{NZ}) / \mathrm{FNZT}$

$A D D I=A D D I+S D D I(N Z) / F N Z T$

$A D P R=A D P R+S D P R(N Z) / F N Z T$

$A D P I=A D P I+S D P I(N Z) / F N Z T$

125 CONTINUE

135 $\mathrm{ZDTRO}=\mathrm{ZDF} * \mathrm{SZDR}$

ZDTI0 $=\mathrm{ZDF} *($ SAIR $1+S Z D I)$

$\mathrm{ZPTR} 0=\mathrm{ZPF} * \mathrm{SZPR}$

$Z P T I 0=Z P F *(S A I R 2+S Z P I)$

ZMTRO $=\mathrm{ZMF} * \mathrm{SZMR}$

$Z M T I O=Z M F * S Z M I$

$\mathrm{ZMTDR}=-\mathrm{ZMF} * \mathrm{DMF} *(\mathrm{ADDR} * \mathrm{ADPR}-\mathrm{ADDI} * \mathrm{ADPI})$

$\mathrm{ZMTDI}=-\mathrm{ZMF} * \mathrm{DMF} *(\mathrm{ADDI} * \mathrm{ADPR}+\mathrm{ADDR} * \mathrm{ADPI})$

C. DEFINE COMPLEX QUANTITIES, DO COMPLEX CIRCUIT CALCULATIONS ZDT $=$ DCMPLX $($ ZDTR , ZDTI $)$

ZPT=DCMPLX (ZPTR, ZPTI) 
ZMT $=$ DCMPLX (ZMTR, ZMTI)

1000 CONTINUE

SRHSR $=$ ZMTDR $*$ DELTAR I SRIISR

SRHS I $=$ ZMTDI $*$ DELTAR+SRHS I

180 FORMAT (F6. 3, 1X, D11.3, 1X, F7.2)

C 181 FORMAT(F6.3, 2X, D11.3, 3X, F7.2)

1010 CONTINUE

$\mathrm{EBW}=\mathrm{Z} 6 * \mathrm{Z} 7 * \mathrm{ZMTO} * \mathrm{ZMT} 0+(\mathrm{Z} 6 *(\mathrm{ZDT} 0+\mathrm{R} 6)+\mathrm{Z} 0) *(\mathrm{Z} 7 *(\mathrm{ZPT} 0+\mathrm{R} 7)+\mathrm{Z} 9)$

RHSC $=$ VIN $*$ R $9 *$ GAIN $*$ DCMPLX $($ SRHSR , SRHSI $) / E B W$

RHSR $=$ REAL (RHSC)

RHSI=DIMAG (RHSC)

$\mathrm{RHSM}=\mathrm{DS} \mathrm{SRT}(\mathrm{RHSR} * \mathrm{RHSR}+\mathrm{RHSI}$ *RHSI$)$

RHSP=DATAN2 (RHSI, RHSR) $* 180$. /PI

WRITE (LOD , 180) ZD2, RHSM, RHSP

WRITE (LOU, 180) ZD2, RHSM, RHSP

WRITE $(0,180)$ ZD2, RHSM, RHSP

1020 CONTINUE

1040 END 
RFDSF calculates mag. and phase of DSF for a lattice of points

Program RFDSF calculates the magnitude and phase of the defect sensitivity factor of a reflection coil at a lattice of points throughout a conducting plate, as shown in Fig. 11.

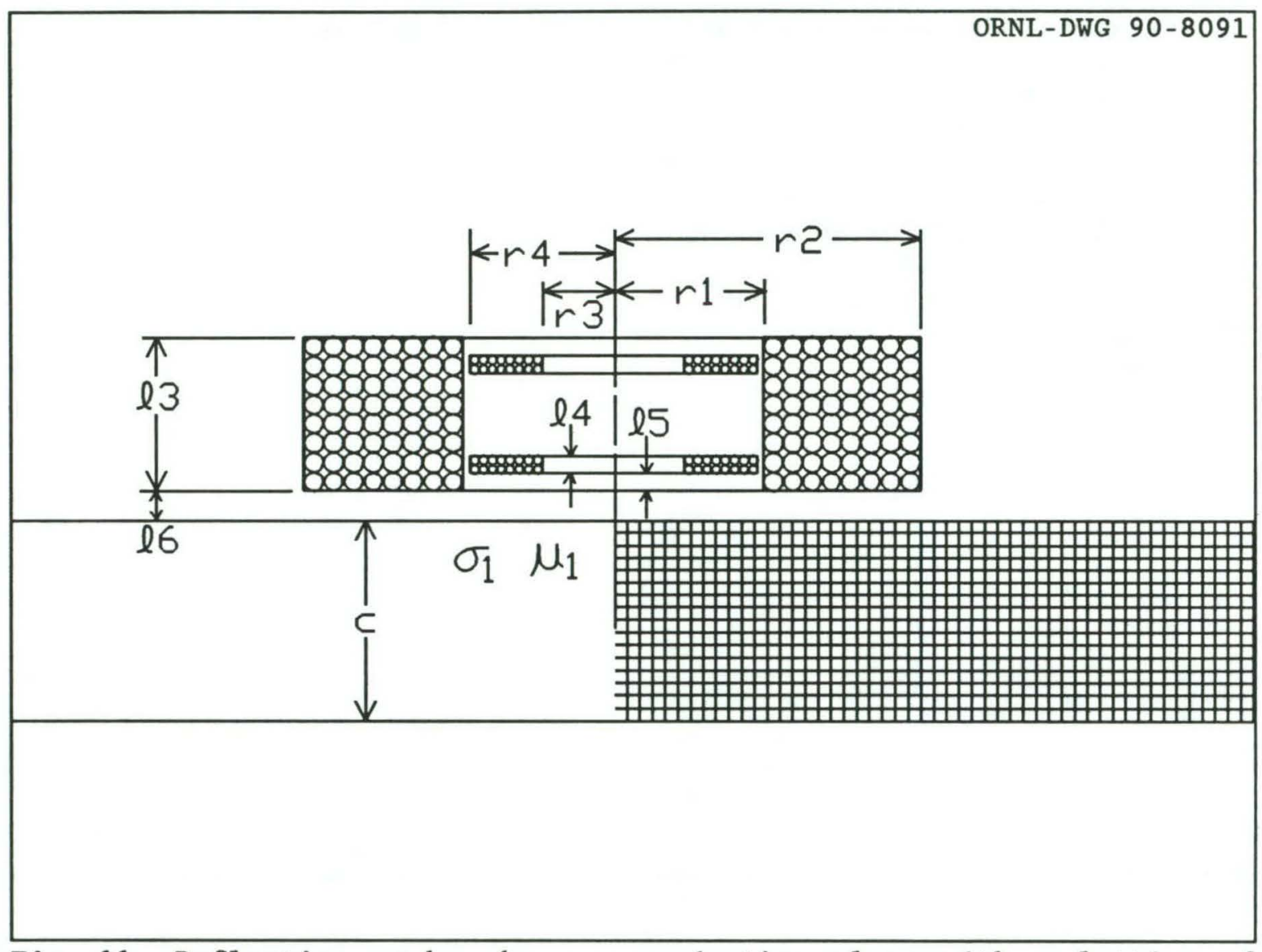

Fig. 11. Reflection probe above a conducting plane with a lattice of points.

The defect sensitivity is the mutual coupling term between the driver coil and pickup coils due to the defect in Eq. (26), and is given by: 


$$
\begin{aligned}
& D S F(r, z)=\frac{3 \omega \mu \sigma \overline{\mathrm{r}}^{2}}{8 \pi}\left[\int_{0}^{\infty} \frac{e^{-\alpha l_{6}}}{\alpha^{3}} J\left(r_{2}, r_{1}\right)\left(1-e^{-\alpha l_{3}}\right) J_{1}(\alpha r) F\left(\alpha, \alpha_{1}, z\right) d \alpha\right] \times \\
& \left.\left[\int_{0}^{\infty} \frac{e^{-\alpha\left(l_{6}+l_{5}\right)}}{\alpha^{3}} J\left(r_{4}, r_{3}\right)\left(1-e^{-\alpha\left(l_{3}-l_{4}-2 l_{5}\right)}\right)\left(1-e^{-\alpha l_{4}}\right) J_{1}(\alpha r) F\left(\alpha, \alpha_{1}, z\right) d \alpha\right]\right\}
\end{aligned}
$$

As we can see from the circuit equation, (20), the mutual impedance term is not exactly equal to the voltage change due to the defect, but it is the dominant term. The defect sensitivity magnitudes and phases are stored in a file named FORT40 so they can be plotted by program RFDSFPLT.

\section{Summary}

1. Dimension arrays and declare variable types. Initialize variables.

2. Write the coil and plate information to a data file.

3. Select a point at which to calculate the defect sensitivity factor by choosing a value for $\mathrm{RD}$, the radial distance from the axis of the coil, and for $\mathrm{ZD}$, the distance of the point from the near side of the plate.

4. Perform the integration necessary to calculate the defect sensitivity factor at this point.

5. Store the calculations in a file.

6. Loop to 3 until done.

\section{$\underline{\text { Variables }}$}

NOTE: Most of the variables which occur inside the integration loops are uninteresting because they do not correspond to anything physical and because they should never require user intervention. Therefore, these variables will not be discussed here. For a detailed description of these variables, see the documentation of program RFAVZSCN. For a discussion of the major differences between the integration section of program RFDSF and the integration section of program RFAVZSCN, see note \#1.

Starred variables must be set by the user.

C6 $6^{\circ} \quad$ The total shunt capacitance in farads of the driving circuit.

C7 The total shunt capacitance in farads of the pickup circuit.

DELTAR The normalized distance in the radial direction between adjacent data points.

DELTAZ The normalized distance in the axial direction between adjacent data points.

FREQ $\quad$ The operating frequency in hertz.

L3

The normalized length of the driver coil.

L4

The normalized length of the pickup coil. 


\begin{tabular}{|c|c|}
\hline 5 & The normalized distance of recess of the pickup \\
\hline & The normalized lift-off of the driver coil. \\
\hline $\mathrm{OD}^{\circ}$ & $\begin{array}{l}\text { The channel on which the output data file is } \\
\text { opened. }\end{array}$ \\
\hline NPROBE ${ }^{\circ}$ & $\begin{array}{l}\text { Character variable which contains the name of the } \\
\text { reflection probe which is to be used in the } \\
\text { calculations. }\end{array}$ \\
\hline NRT $^{*}$ & $\begin{array}{l}\text { The total number of points in the radial direction } \\
\text { at which the defect sensitivity factor is } \\
\text { calculated. }\end{array}$ \\
\hline NZT* & $\begin{array}{l}\text { The total number of points in the axial direction } \\
\text { at which the defect sensitivity factor is } \\
\text { calculated. }\end{array}$ \\
\hline $\mathrm{RO}^{\circ}$ & $\begin{array}{l}\text { Output series resistance of driving amplifier in } \\
\text { ohms. }\end{array}$ \\
\hline & The normalized inner radius of the driver coil. \\
\hline & The normalized outer radius of the driver coil \\
\hline & The normalized inner radius of the pickup coil \\
\hline 24 & The-normalized outer radius of the pickup coil \\
\hline 25 & The mean radius of the driver coil in inches. \\
\hline 26 & DC resistance of the driver coil in ohms. \\
\hline 27 & DC resistance of the pickup coil in ohms. \\
\hline $29^{\circ}$ & The amplifier input impedance. \\
\hline $\mathrm{HO}^{\circ}$ & The resistivity in $\mu \Omega-\mathrm{cm}$ of the plate. \\
\hline & $\begin{array}{l}\text { The thickness of the plate. When it first occurs, } \\
\text { it is in inches, but it is normalized by the } \\
\text { program. }\end{array}$ \\
\hline & The density of turns in the driver coil. \\
\hline & The density of turns in the pickup coil. \\
\hline & The number of turns in the driver coil. \\
\hline & The number of turns in the pickup coil. \\
\hline & he relative magnetic permeability of $t$ \\
\hline
\end{tabular}

\section{Notes}

1. The integration in this program is very similar to the integration in program RFAVZSCN, but some very minor differences do exist. Most of these come from the fact that this program calculates only the defect sensitivity factor, which does not depend on the volume of the defect, and program RFAVZSCN calculates the voltage change due to the defect, which does depend on the volume of the defect. While it is necessary to calculate the defect sensitivity factor to calculate the voltage change due to the defect, program RFAVZSCN never calculates the defect sensitivity factor as a separate quantity. Thus, some of the variables in RFAVZSCN which have the same name as variables in program RFDSF have an extra factor of the defect volume. This program does carry and calculate some quantities that are not directly used by this program but are used by similar programs. The calculation of these variables does not add any significant running time to the program. 
Sample output

Printer output of program RF̄DSF:

RFDSF TIME $9: 24: 38$ DATE $8 / 9 / 89$

PROBE 250A PLATE THIK 0:2500

COIL IN RAD OT RAD LENGTH OLO/REC TURNS COIL RES CKT: RES CAP

DRIVER $0.7500 \quad 1.2500 \quad 0.6000 \quad 0.0600 \quad 2350.0 \quad 5.310 \mathrm{E}+02 \quad 3.050 \mathrm{E}+03 \quad 8.470 \mathrm{E}-11$

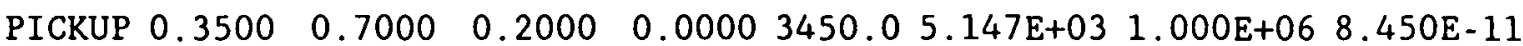

RBAR 0.2500 FREQ $=5.000000 E+02 \mathrm{RHO}=4.0540 \mathrm{PERM}=1.000$ WUSRR $=3.9267$

Partial listing of file FORT40:

\begin{tabular}{|c|c|c|}
\hline 25 & 20 & \\
\hline 0.08000 & \multicolumn{2}{|l|}{0.05263} \\
\hline 0.75000 & \multicolumn{2}{|l|}{1.25000} \\
\hline 0.35000 & \multicolumn{2}{|l|}{0.70000} \\
\hline 0.60000 & \multicolumn{2}{|l|}{0.20000} \\
\hline 0.06000 & \multirow{2}{*}{\multicolumn{2}{|c|}{0.00000}} \\
\hline 1.00000 & & \\
\hline 1 & $0.11353 \mathrm{D}-04$ & $-0.46336 \mathrm{D}+00$ \\
\hline 2 & $0.97720 \mathrm{D}-05$ & $-0.53972 D+00$ \\
\hline 3 & $0.81151 D-05$ & $-0.61645 D+00$ \\
\hline 4 & $0.65622 \mathrm{D}-05$ & $-0.69443 D+00$ \\
\hline 5 & $0.52051 D-05$ & $-0.77420 D+00$ \\
\hline 6 & $0.40726 \mathrm{D}-05$ & $-0.85603 D+00$ \\
\hline 7 & $0.31569 \mathrm{D}-05$ & $-0.94004 D+00$ \\
\hline 8 & $0.24324 D-05$ & $-0.10262 D+01$ \\
\hline 9 & $0.18679 \mathrm{D}-05$ & $-0.11143 D+01$ \\
\hline & $4326 \mathrm{D}-05$ & $-0.12040 D+01$ \\
\hline
\end{tabular}




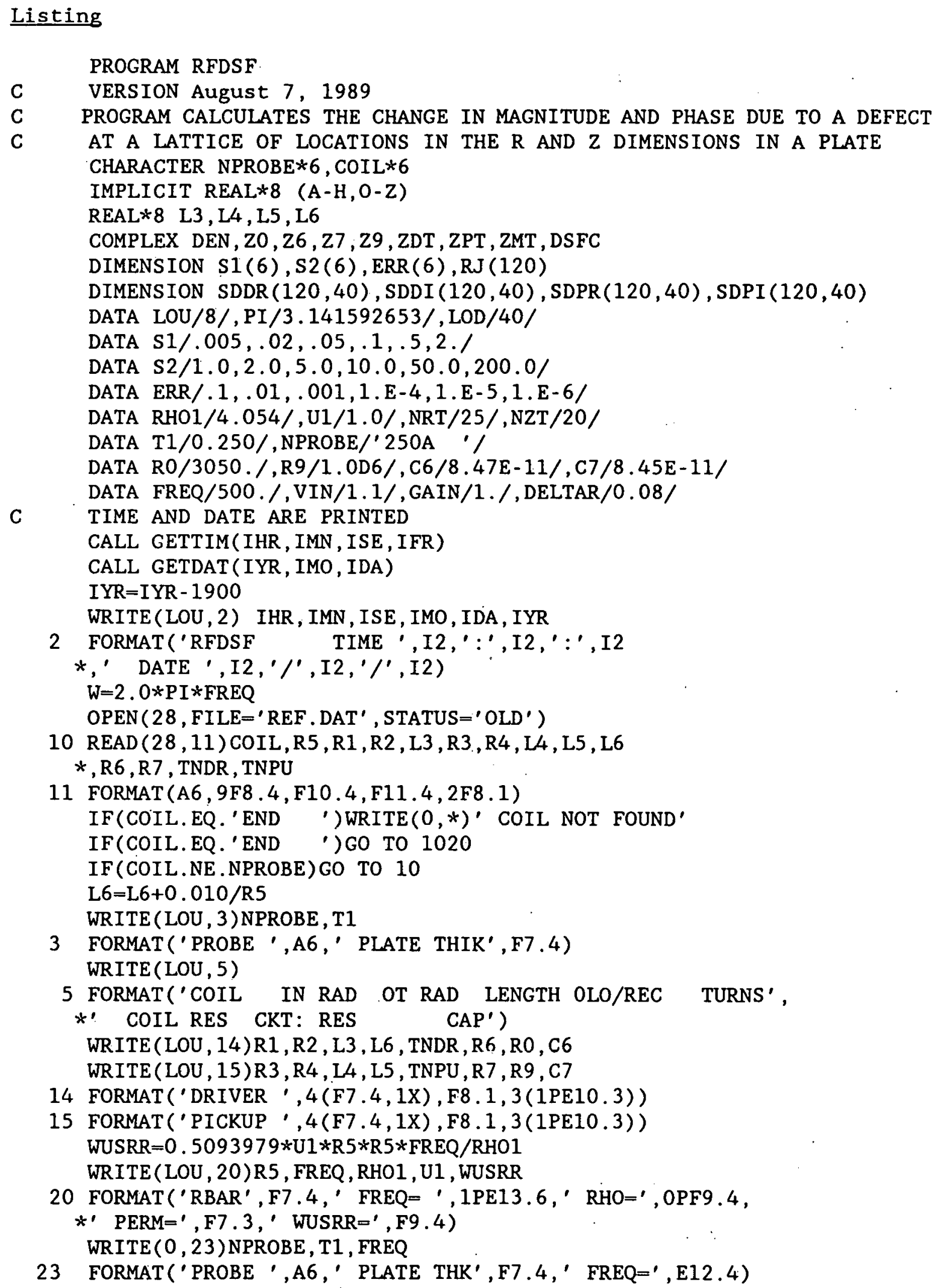




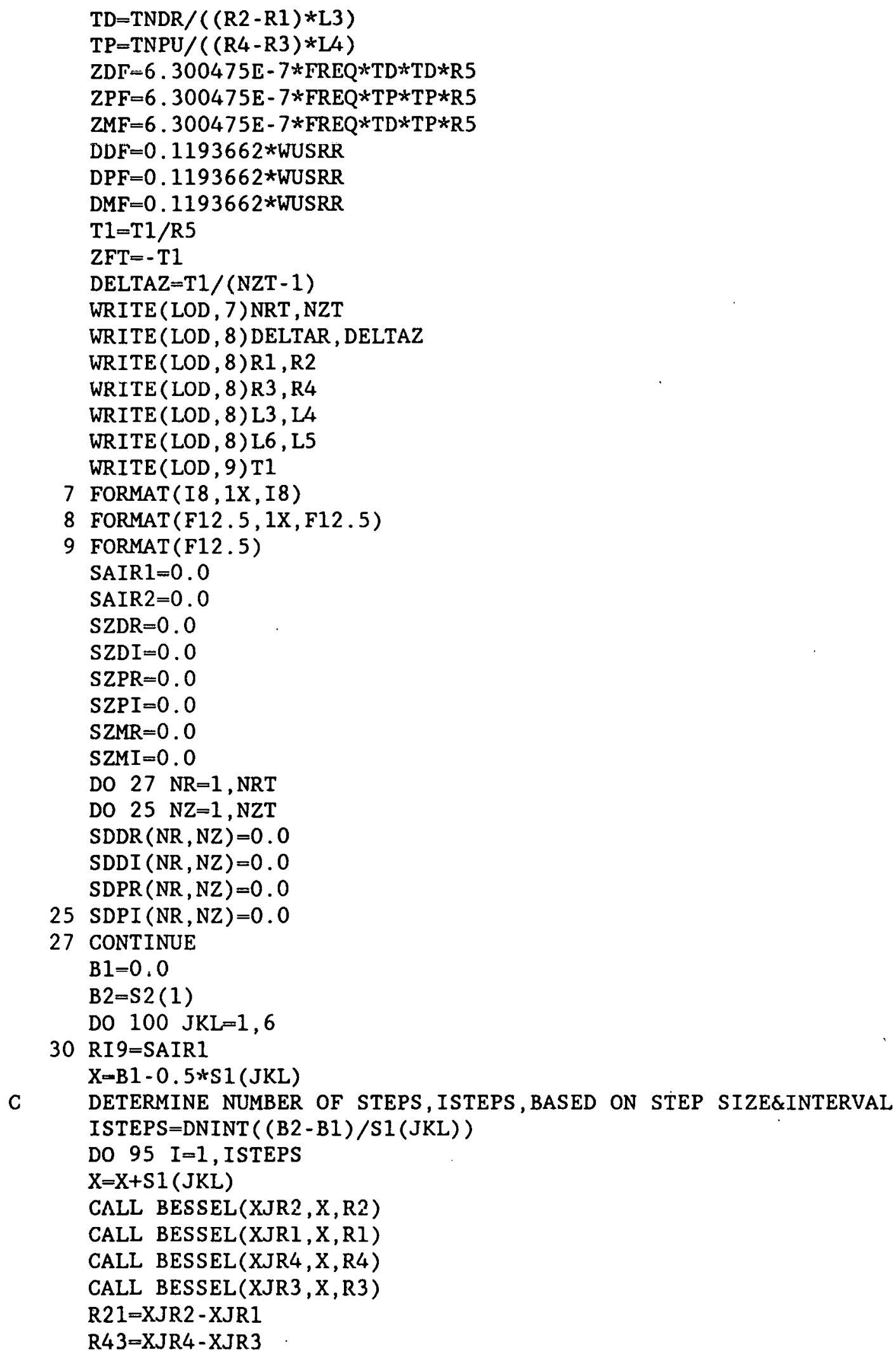


$\mathrm{XL} 3=\mathrm{X} * \mathrm{~L} 3$

IF (XL3.GT.5.0E-3) GO TO 60

$\mathrm{A} 1=\mathrm{XL} 3 * \mathrm{XL} 3 *(0.5-\mathrm{XL} 3 / 6.0)$

GO TO 80

60 IF(XL3.GT.75.0) GO TO 70

$\mathrm{A} 1=\mathrm{XL} 3+\mathrm{DEXP}(-\mathrm{XL} 3)-1.0$

GO TO 80

$70 \mathrm{~A} 1=\mathrm{XL} 3-1.0$

80 A3 $=X L 3-A 1$

$\mathrm{SFD}=\mathrm{S} 1(\mathrm{JKL}) * \mathrm{R} 21 * \mathrm{R} 21$

$\mathrm{SFP}=\mathrm{S} 1(\mathrm{JKL}) * \mathrm{R} 43 * \mathrm{R} 43$

$S F M=S 1(J K L) * R 21 * R 43$

SAIR $1=S A I R 1+S F D * 2.0 * A 1$

$\mathrm{XL} 4=\mathrm{X} * \mathrm{~L} 4$

IF(XL4.GT.5.0E-3) GO TO 81

$\mathrm{A} 2=\mathrm{XL} 4 \times \mathrm{XL} 4 *(0.5-\mathrm{XL} 4 / 6.0)$

GO TO 83

81 IF(XL4.GT.75.0) GO TO 82

$\mathrm{A} 2=\mathrm{XL} 4+\mathrm{DEXP}(-\mathrm{XL} 4)-1.0$

GO TO 83

82 A2 $=$ XL4 -1.0

83 A4 $=X L 4-A 2$

$A 13=1.0-A 3$

A14 $=1.0-A 4$

$\mathrm{A} 5=\mathrm{DEXP}(-\mathrm{X} * \mathrm{~L} 5)$

$\mathrm{XL} 8=\mathrm{X} *(\mathrm{~L} 3-2 . * \mathrm{~L} 4-2 . * \mathrm{~L} 5)$

IF (XL8.GT. 75.) A8=0.

IF (XL8 . LE. 75.) A8 $=$ DEXP ( $-X L 8)$

SAIR2 $=$ SAIR2+SFP* $(4.0 * A 2-2.0 * A 4 * A 4 * A 8)$

IF $(X *$ L6.GT. 75. ) GO TO 95

C

$\mathrm{XX}=\mathrm{X} * \mathrm{X}$

$\mathrm{X} 1=\mathrm{DSQRT}(0.5 *(\mathrm{XX}+\mathrm{DSQRT}(\mathrm{XX} * \mathrm{XX}+\mathrm{WUSRR} * \mathrm{WUSRR}))) / \mathrm{U} 1$

$\mathrm{Y} 1=$ WUSRR $/(2 . * \mathrm{X} 1 * \mathrm{U} 1 * \mathrm{U} 1)$

$\mathrm{A} 6=\mathrm{DEXP}(-\mathrm{X} * \mathrm{~L} 6)$

$\mathrm{XL7}=\mathrm{X} *(\mathrm{~L} 3-\mathrm{L} 4-2 . * \mathrm{~L} 5)$

IF (XL7.GT. 75.)A7=1.0

IF (XL7.LE. 75.)A7 $=1.0-\operatorname{DEXP}(-X L 7)$

$\mathrm{A} 9=\mathrm{A} 4 * \mathrm{~A} 5 * \mathrm{~A} 6 * \mathrm{~A} 7$

$\mathrm{APBR}=(\mathrm{X}+\mathrm{X} 1) *(\mathrm{X}+\mathrm{X} 1)-\mathrm{Y} 1 * \mathrm{Y} 1$

$A P B I=2 * Y 1 *(X+X 1)$

$A M B R=(X-X 1) *(X-X 1)-Y 1 * Y 1$

$A M B I=-2 . * Y 1 *(X-X 1)$

$\mathrm{A} 2 \mathrm{BR}=0.0$

$A 2 B I=-2 . * X 1 * Y 1$

$\mathrm{ZNUR}=\mathrm{A} 2 \mathrm{BR}$

$\mathrm{ZNUI}=\mathrm{A} 2 \mathrm{BI}$

$\mathrm{DENR}=\mathrm{APBR}$

DENI $=A P B I$

$D N C J=D E N R * D E N R+D E N I * D E N I$

$\mathrm{C}$

SECTION THAT MULTIPLIES BY DEXP(ALPHA $1 * Z D E F E C T)$ 
DO $91 \mathrm{NZ}=1, \mathrm{NZT}$

$\mathrm{FZD}=(\mathrm{NZ}-\mathrm{I}) * \mathrm{ZFT} /(\mathrm{NZT}-1)$

$\mathrm{ZDR}=\mathrm{X} 1 * \mathrm{U} 1 * \mathrm{FZD}$

IF(ZDR. LT . -60.0)GO TO 93

$\mathrm{ZDI}=\mathrm{Y} 1 * \mathrm{U} 1 * \mathrm{FZD}$

$X P D R=D E X P(Z D R)$

$C S D I=D C O S(Z D I) * X P D R$

SNDI $=D S I N(Z D I) * X P D R$

$\mathrm{XX} 1=\mathrm{X} * \mathrm{X} 1+\mathrm{XX}$

$\mathrm{XY} 1=\mathrm{X} * \mathrm{Y} 1$

$\mathrm{X} 1 \mathrm{X}=\mathrm{X} * \mathrm{X} 1-\mathrm{XX}$

$\mathrm{ZNDR}=\mathrm{XX} 1 * \mathrm{CSDI}-\mathrm{XY} 1 * \mathrm{SNDI}$

$\mathrm{ZNDI}=\mathrm{XX} 1 * \mathrm{SNDI}+\mathrm{XY} 1 * \mathrm{CSDI}$

C

SECTION THAT MULTIPLIES BY DEXP (ALPHA. $1 *(2 *$ TH+ZDEFECT $))$

$\mathrm{TZR}=\mathrm{X} 1 * \mathrm{U} 1 *(2 . * \mathrm{~T} 1+\mathrm{FZD})$

IF(TZR.GT.60.) GO TO 87

$\mathrm{TZI}=\mathrm{Y} 1 * \mathrm{U} 1 *(2 . * \mathrm{~T} 1+\mathrm{FZD})$

$X P Z R=D E X P(-T Z R)$

$\mathrm{CSZI}=\mathrm{DCOS}(\mathrm{TZI}) * X P Z R$

$S N Z I=D S I N(T Z I) * X P Z R$

$\mathrm{ZNDR}=\mathrm{XXI} 1 * \mathrm{CSDI}-\mathrm{XY} 1 * \mathrm{SNDI}+\mathrm{X} 1 \mathrm{X} * \mathrm{CSZI}+\mathrm{XY} 1 * \mathrm{SNZI}$

$\mathrm{ZNDI}=\mathrm{XXI} 1 * \mathrm{SNDI}+\mathrm{XY} 1 * \mathrm{CSDI}+\mathrm{XY} 1 * \mathrm{CSZI}-\mathrm{XIX} * \mathrm{SNZI}$

C SECTION THAT MULTIPLIES BY DEXP (-ALPHA $1 * 2 *$ CLADTH)

$\mathrm{TR}=2 . * \mathrm{X} 1 * \mathrm{U} 1 * \mathrm{~T} 1$

IF(TR.GT.60.) GO TO 87

$\mathrm{TI}=2 . * \mathrm{Y} 1 * \mathrm{U} 1 * \mathrm{~T} 1$

$\mathrm{XPTR}=\mathrm{DEXP}(-\mathrm{TR})$

C.STT $=$ DS,NS $(T T) * X P T R$

SNTI $=D S I N(T I) * X P T R$

DENR-APBR - AMBR *CSTI - AMBI $* S N T I$

$\mathrm{DENI}=\mathrm{APBI}-\mathrm{AMBI} * \mathrm{CSTI}+\mathrm{AMBR} * \mathrm{SNTI}$

$\mathrm{ZNUR}=\mathrm{A} 2 \mathrm{BR}-\mathrm{A} 2 \mathrm{BR} * \mathrm{CSTI}-\mathrm{A} 2 \mathrm{BI} * \mathrm{SNTI}$

$\mathrm{ZNUI}=\mathrm{A} 2 \mathrm{BI}-\mathrm{A} 2 \mathrm{BI} * \mathrm{CSTI}+\mathrm{A} 2 \mathrm{BR} * \mathrm{SNTI}$

$\mathrm{DNCJ}=\mathrm{DENR} * \mathrm{DENR}+\mathrm{DENI} * \mathrm{DENI}$

$87 \mathrm{ZDRL}=(\mathrm{ZNDR} * \mathrm{DENR}+\mathrm{ZNDI} * \mathrm{DENI}) / \mathrm{DNCJ}$

$Z D I M=(D E N R * Z N D I-Z N D R * D E N I) / D N C J$

C LOOP OVER THE R VARIATION FOR THE DEFECI

DO $90 \mathrm{NR}=1$, NRT

IF(NZ.GT.1) GO TO 89

$\mathrm{RD}=\mathrm{FLOAT}(\mathrm{NR}) * \mathrm{DELTAR}$

$\mathrm{XRD}=\mathrm{X} * \mathrm{RD}$

CALL BESEL1 (XRD, RJ1)

$\mathrm{RJ}(\mathrm{NR})=\mathrm{RJ} 1$

C SUM DEFECT SENSITIVITY FACIORS FOR THE DRIVER \& PICKUP COILS

$89 \operatorname{SDDR}(\mathrm{NR}, \mathrm{NZ})=\mathrm{SDDR}(\mathrm{NR}, \mathrm{NZ})+\mathrm{A} 3 * \mathrm{~A} 6 * \mathrm{RJ}(\mathrm{NR}) * 2 * \mathrm{ZDRL} * \mathrm{R} 21 * \mathrm{~S} 1(\mathrm{JKL})$

$\operatorname{SDDI}(\mathrm{NR}, \mathrm{NZ})=\mathrm{SDDI}(\mathrm{NR}, \mathrm{NZ})+\mathrm{A} 3 * \mathrm{~A} 6 * \mathrm{RJ}(\mathrm{NR}) * 2 * \mathrm{ZDIM} * \mathrm{R} 21 * \mathrm{~S} 1(\mathrm{JKL})$

$\operatorname{SDPR}(\mathrm{NR}, \mathrm{NZ})=\mathrm{SDPR}(\mathrm{NR}, \mathrm{NZ})+\mathrm{A} 9 * \mathrm{RJ}(\mathrm{NR}) * 2 * \mathrm{ZDRL} * \mathrm{R} 43 * \mathrm{~S} 1(\mathrm{JKL})$

$90 \operatorname{SDPI}(\mathrm{NR}, \mathrm{NZ})=\operatorname{SDPI}(\mathrm{NR}, \mathrm{NZ})+\mathrm{A} 9 * \mathrm{RJ}(\mathrm{NR}) * 2 * \mathrm{ZD} . \mathrm{IM} * \mathrm{R} 43 * \mathrm{SI}(\mathrm{JKL})$

91 CONTINUE

$93 \mathrm{ZRL}=(\mathrm{ZNUR} * \mathrm{DENR}+\mathrm{ZNUI} * \mathrm{DENI}) / \mathrm{DNCJ}$

$\mathrm{ZIM}=(\mathrm{DENR} * \mathrm{ZNUI}-\mathrm{ZNUR} * \mathrm{DENI}) / \mathrm{DNCJ}$ 


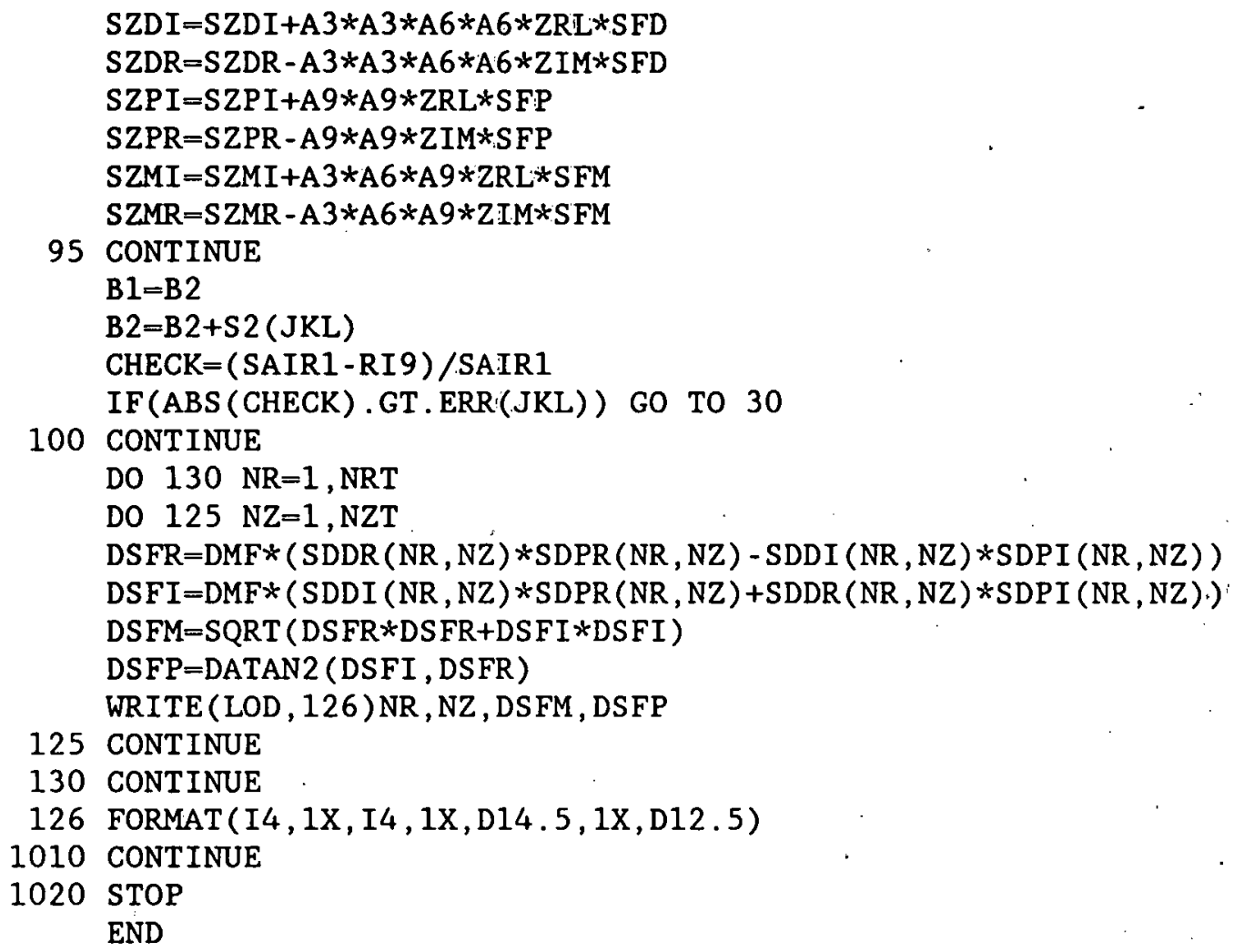


RFDSFPLT generates a contour plot of magnitude of DSF

Program RFDSFPLT generates a contour plot of the magnitude of the defect sensitivity factor for a reflection coil using calculations performed and stored by program RFDSF.

Summary

1. Declare arrays and variable types. Initialize variables.

2. Open the file created by program RFDSF.

3. Read in the information about the coil and the plate from the data file.

4. Calculate the position in the normalized coordinate system (see note \#1) of the data points which are about to be read in.

5. Set the label flags for the contours.

6. Read the data stored by program RFDSF into array DSFMA.

7. Specify the values of the magnitude of the defect sensitivity factor where the contours are to be drawn.

8. Call the PRINTMATIC contour initialization routines.

9. Draw the contours.

10. Draw the coils and the plate.

\section{Variables}

Starred variables must be set by the user.

$\mathrm{CNM}^{\circ} \quad$ Real array which contains the values of the magnitude of the defect sensitivity factor on the contour lines. It is used as input for routine DCNTOUR.

DELTAR The normalized distance in the radial direction between adjacent data points.

DELTAZ The normalized distance in the axial direction between adjacent data points.

DSFMA Two-dimensional real array which is used to hold the values of the magnitude of the defect sensitivity factor which are read in from a data file. Array DSFMA is used as input for routine DINIT.

L3 The normalized length of the driver coil.

L4 The normalized length of the pickup coil.

L5 The normalized distance of recess of the pickup coil.

I.6 The normalized lift-off of the driver coil.

LBM $\quad$ Integer array that specifies which of the contours are to be labeled with their values. If all elements of LBM are zero, none of the contours will be labeled. Array LBM is used as input for routine DCNTOUR.

LOE $\quad$ The channel on which the file created by program 


\begin{tabular}{|c|c|}
\hline NAME ${ }^{*}$ & $\begin{array}{l}\text { RFDSF is opened. } \\
\text { Character variable which contains the name of the } \\
\text { file that program RFDSFPL uses for output. NAME } \\
\text { is used as input for routine DCNTOUR. }\end{array}$ \\
\hline NC ${ }^{*}$ & $\begin{array}{l}\text { Specifies the number of contours to be drawn. The } \\
\text { value of } \mathrm{NC} \text { must be less than or equal to } 10 \text {. It } \\
\text { is used as input for routine DCNTOUR. }\end{array}$ \\
\hline NRT & $\begin{array}{l}\text { The number of points in the radial direction at } \\
\text { which calculations were performed by program } \\
\text { RFDSF. }\end{array}$ \\
\hline NZT & $\begin{array}{l}\text { The number of points in the axial direction at } \\
\text { which calculations were performed by program } \\
\text { RFDSF. }\end{array}$ \\
\hline R1 & The normalized inner radius of the driver coil. \\
\hline $\mathrm{R} 2$ & The normalized outer radius of the driver coil. \\
\hline R3 & The normalized inner radius of the pickup coil. \\
\hline R4 & The normalized outer radius of the pickup coil. \\
\hline $\mathrm{T} 1$ & The normalized thickness of the plate. \\
\hline $\mathrm{XX}$ & $\begin{array}{l}\text { Real array which describes the radial position of } \\
\text { the data points in array DSFMA in the normalized } \\
\text { coordinate system. It is used as input for } \\
\text { routine DCNTOUR. }\end{array}$ \\
\hline YY & $\begin{array}{l}\text { Real array which describes the axial position of } \\
\text { the data points in array DSFMA in the normalized } \\
\text { coordinate system. It is used as input for } \\
\text { routine DCNTOUR. }\end{array}$ \\
\hline
\end{tabular}

Notes

1. The coordinate system set up and used by this program has its origin at the intersection of the coil axis and the near side of the plate. One unit of distance in the coordinate system is equal to one mean radius of the driver coil.

2. The array DSFMA must be dimensioned to exactly NRT by NZT. Each time the value of NRT or NZT is changed in program RFDSF, the statement dimensioning the array in program RFDSFPLT must be changed also.

3. The statements in this program which seem to do nothing but write variables to the screen actually have a more important function. Due to a bug in either the PRINTMATIC routines or in RM/FORTRAN, the PRINTMATIC routine DLINE, which is supposed to draw a straight line, sometimes refuses to work. It was discovered by accident that putting a WRITE statement near the call to the routine corrects the problem.

4. Program RFDSFPLT does not actually send anything to the printer; it merely creates a file whose name is given by the program variable NAME. If the value of NAME is 'filename.ext', then to print the file created by program RFDSFPLT, enter DPRINT filename.ext

DPRINT.EXE is a program supplied by PRINTMATIC. 


\section{Sample Output}

Fig. 12 shows a typical plot generated by program RFDSFPLT.

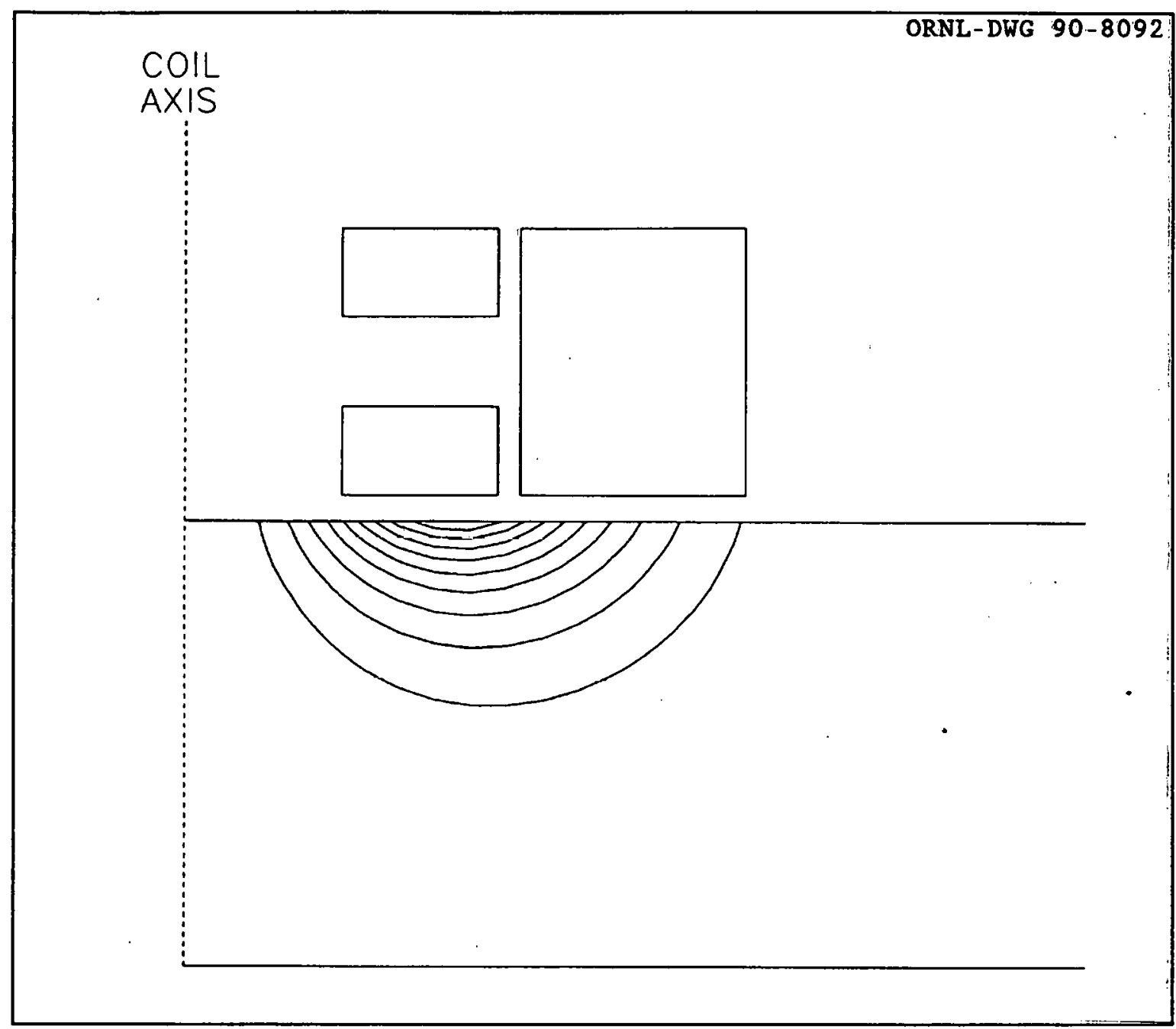

Fig. 12. Contour map of a the defect sensitivity factor for a reflection probe above a conducting plate. 


\section{Listing}

\section{PROGRAM RFDSFPLT}

C VERSION October 31,1988

C Program to generate a contour plot of the magnitude of the

C defect sensitivity factor of a reflection coil.

C

\section{CHARACTER $* 80$ NAME}

IMPLICIT REAL*4 (A-H, O-Z)

REAL*4 DSFMA $(25,20)$

REAL *4 XX(25), YY(20)

REAL *4 CNM(10)

$\mathrm{REAL} * 4 \mathrm{~L} 3, \mathrm{~L} 4, \mathrm{~L} 5, \mathrm{~L} 6$

INTEGER $* 2$ LBM(10)

INTEGER $* 2 \mathrm{I} 1, \mathrm{~J} 1, \mathrm{I} 2, \mathrm{~J} 2$

DATA XSCALE/1.0/,NC/9/

DATA IDEF/2/,LOE $/ 40 /$

\section{c}

C Open the file created by program RFDSF and read in the

C coil and plate information.

C

$$
\text { OPEN( LOE , FILE=' FORT40', STATUS=' OLD' })
$$

READ (LOE , *)NRT, NZT

READ $($ LOE, *) DELTAR, DELTAZ

READ $($ LOE , *) R1, R2

$\operatorname{READ}($ LOE , *) R3, R4

$\operatorname{READ}(\mathrm{LOE}, *) \mathrm{L} 3, \mathrm{~L} 4$

READ $($ LOE , *) L6, L5

$\operatorname{READ}(\operatorname{LOE}, *) \mathrm{T} 1$

C:

C. Calculate the position of the data points in the

C normalized coordinate system.

C

D0 $110 \quad I=1, N R T$

$\mathrm{XX}(\mathrm{I})=\mathrm{REAL}(\mathrm{I}) * \mathrm{DELTAR}$

110 CONTINUE

DO $120 I=0, N Z T-1$

$Y Y(I+1)=-(((N Z T-1)-R E A L(I)) *$ DELTAZ $)$

120 CONTINUE

C

C) Set the label flags for the contours.

C:

DO) $130 \quad I=1,10$

$\mathrm{LBM}(\mathrm{I})=0$

130 CONTINUE

C:

C. Read in the data stored by program RFDSF.

C.

1.40. READ ( LOE , * END $=150$ ) NR, NZ, DSFM

$\mathrm{NZ}=\mathrm{NZT}-\mathrm{NZ}+1$

IF (DSFM . GT . DSFMMAX) DSFMMAX $=\mathrm{DSFM}$ 


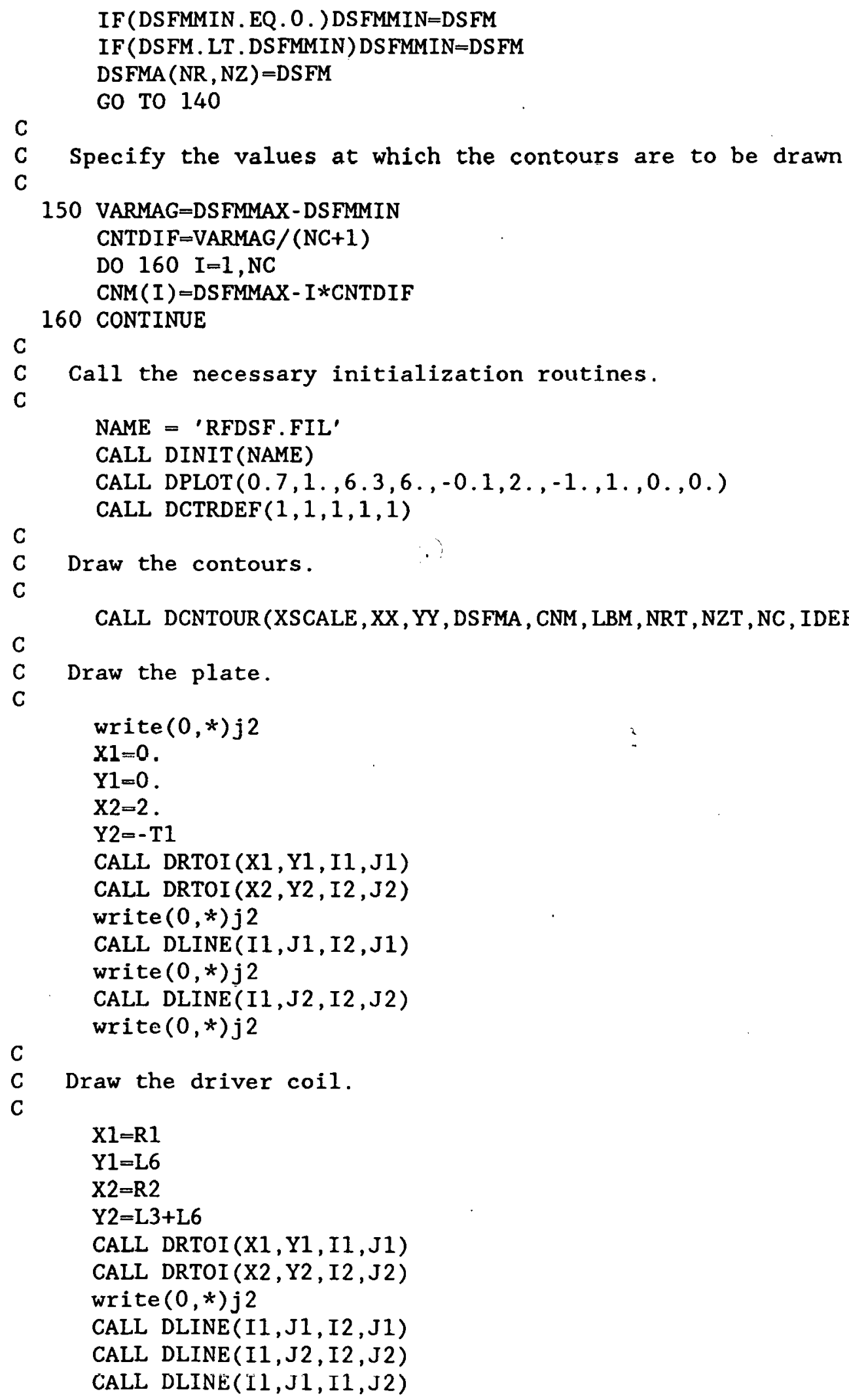




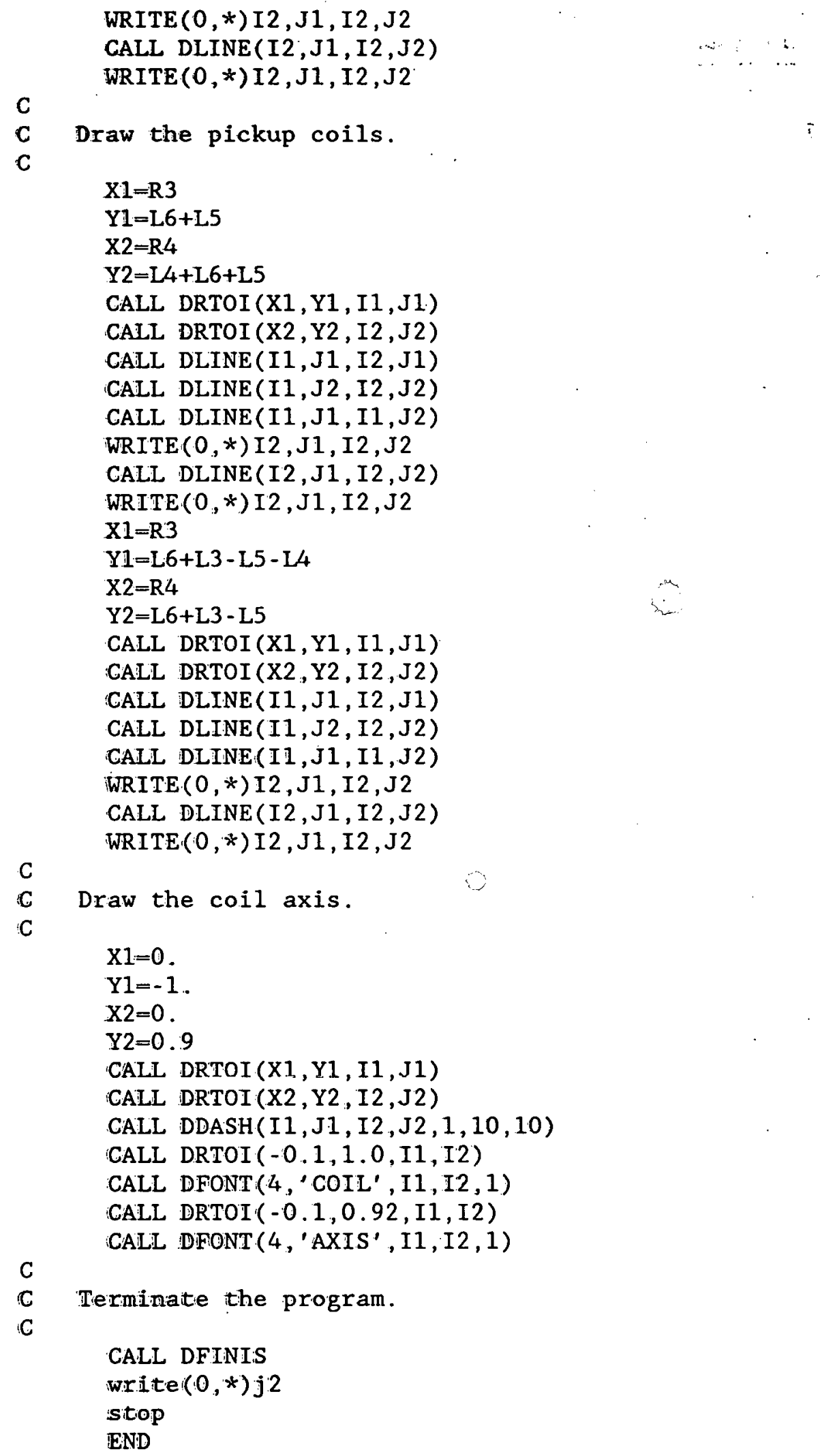


RFAVZSCN calculates defect voltage change, average over depth

Program RFAVZSCN calculates the change in the induced voltage in a pickup coil due to the presence of a defect in a plate. It does the calculations for a number of different coil-to-defect distances (see note \#1), and it has the ability to divide the defect into a number of parts along the depth of the defect, centered on the axis of symmetry of the defect and to perform the calculations for each part separately, averaging the results to achieve better agreement with experimental results than if the defect were treated as a whole (see note \#2). The program will scan the coil across the defect in the $r$ direction and calculate the magnitude and phase of the defect. It is now set up to scan from the inner edge of the pickup coil, $r_{3}$, to the outer edge of the driver coil, $r_{2}$. It can be easily changed to any other set of values that are desired. The statement that controls the defect starting and ending point is:

\section{$\mathrm{RD}=\mathrm{R} 3+\mathrm{DELTR} * \mathrm{FLOAT}(\mathrm{NR})$}

This statement must be changed at both its occurrences in the program. The step size, DELTR, is calculated by:

$$
\text { DELTR }=(\text { R2 }- \text { R3 }) / \text { NRT }
$$

The new values for the start and end of the scan should be placed in these equations.

A large section of the program is concerned with doing integrations to find the impedance of the coils. The details of the integration have been placed in a separate section at the end of the discussion.

The output from this program is stored in the file FORT39.

\section{Summary}

1. Dimension arrays and declare variable types.

2. Initialize variables.

3. Calculate the coil impedances in the absence of defects.

4. Select a value for $\mathrm{RD}$, the radial distance between the coil axis and the center of the defect.

5. Do the integrals to calculate the change in coil impedance due to the defect.

6. Calculate the change in voltage due to the defect from the impedance changes.

7. Write the results to a data file.

8. Loop to 4 until. done. 
Variables

Starred variables must be set by the user.

66 The total shunt capacitance in farads of the

C7 $\quad$ The total shunt capacitance in farads of the pickup circuit.

DELTR $\quad$ The normalized distance in the radial direction between adjacent data points.

DFDEP $\quad$ The depth to the bottom of the defect in inches.

DFDIAM $\quad$ The diameter of the defect in inches.

DVVI The imaginary part of the change in induced voltage in the pickup coil due to the defect.

DVVR . The real part of the change in induced voltage in the pickup coil due to the defect.

FNZT Same as variable NZT, but a real variable instead of an integer.

FREQ $\quad$ The operating frequency in hertz.

GAIN ${ }^{*} \quad$ Gain of pickup amplifier.

L3

L4

The normalized length of the driver coil.

The normalized length of the pickup coil.

L5 The normalized distance of recess of the pickup coil.

L6 The normalized lift-off of the driver coil.

LOD ${ }^{\circ} \quad$ The channel on which the output data file is opened.

NPROBE: Character variable which contains the name of the reflection probe which is to be used in the calculations.

NRT' The total number of points in the radial direction at which the defect sensitivity factor is calculated.

NS $\quad$ The side of the plate where the defect is located. If NS $=1$, the defect is on the near side; if NS $=2$, the defect is on the far side.

$\mathrm{NZT}^{*} \quad$ The number of parts into which the defect is divided along its axis to do the calculations.

PHA The phase of the change (not the change of the phase) of the induced voltage in the pickup coil due to the defect.

$\mathrm{RO}^{\circ} \quad$ The output series resistance of the driving amplifier in ohms.

R1 The normalized inner radius of the driver coil.

R2 The normalized outer radius of the driver coil.

R3 The normalized inner radius of the pickup coil.

R4. The normalized outer radius of the pickup coil.

R5 The mean radius of the driver coil in inches.

R6 DC resistance of the driver coil in ohms.

R7 DC resistance of the pickup coil in ohms.

R9' The input shunt resistance of pickup amplifier in 
RD

ohms.

\begin{tabular}{|c|c|}
\hline & $\begin{array}{l}\text { from the axis of the coil to the center of the } \\
\text { defect. (See note } \# 1 \text {.) }\end{array}$ \\
\hline $\mathrm{RHO1}^{\circ}$ & The resistivity of the plate in $\mu \Omega-\mathrm{cm}$. \\
\hline $\mathrm{T} 1^{\circ}$ & $\begin{array}{l}\text { The thickness of the plate. It is input in inch } \\
\text { and normalized by the program. }\end{array}$ \\
\hline $\mathrm{TD}$ & The density of turns in the driver coil. \\
\hline TMAG & $\begin{array}{l}\text { The magnitude of the change (not the change of } t \\
\text { magnitude) in induced voltage in the pickup co } \\
\text { due to the defect. }\end{array}$ \\
\hline TNDR & The number of turns in the driver coil. \\
\hline TNPU & The number of turns in the pickup coil. \\
\hline TP & The density of turns in each pickup coil. \\
\hline $\mathrm{U} 1^{\bullet}$ & The relative magnetic permeability of the plat \\
\hline VIN $^{*}$ & Output voltage of driving amplifier in volts. \\
\hline VOLN & The normalized volume of the defect. \\
\hline VVOI & $\begin{array}{l}\text { The imaginary part of the induced voltage in the } \\
\text { pickup coil in the absence of defects. }\end{array}$ \\
\hline VVOR & $\begin{array}{l}\text { The real part of the induced voltage in the picku } \\
\text { coil in the absence of defects. }\end{array}$ \\
\hline VV1I & $\begin{array}{l}\text { The imaginary part of the induced voltage in the } \\
\text { pickup coil in the presence of a defect. }\end{array}$ \\
\hline VV1R & $\begin{array}{l}\text { The real part of the induced voltage in the pickup } \\
\text { coil in the presence of a defect. }\end{array}$ \\
\hline W & $\begin{array}{l}\text { The angular frequency at which the circuit is } \\
\text { driven. }\end{array}$ \\
\hline ZD & $\begin{array}{l}\text { The normalized distance from the near surface of } \\
\text { the plate to the center of the defect. It is a } \\
\text { negative number. }\end{array}$ \\
\hline ZDTI & $\begin{array}{l}\text { The imaginary part of the total self impedance of } \\
\text { the driver coil. }\end{array}$ \\
\hline ZDTR & $\begin{array}{l}\text { The real part of the total self impedance of the } \\
\text { driver coil. }\end{array}$ \\
\hline ZMTI & $\begin{array}{l}\text { The imaginary part of the total mutual impedance } \\
\text { between the driver and pickup coil. }\end{array}$ \\
\hline 'ZM'I'R & $\begin{array}{l}\text { The real part of the total mutual imperanse. } \\
\text { between the driver and pickup coil. }\end{array}$ \\
\hline ZPTI & $\begin{array}{l}\text { The imaginary part of the total self impedance of } \\
\text { the pickup coil. }\end{array}$ \\
\hline ZPT & $\begin{array}{l}\text { The real part of the total self impedance of the } \\
\text { pickup coil. }\end{array}$ \\
\hline
\end{tabular}

Notes

1. When this program begins, the radial distance RD between the coil axis and the center of the defect is initialized to the value of $R 3$, the pickup coil inner radius, and when the calculations for this value of $R D$ have been completed, the program increases the value of $R D$ and repeats the calculations until $R D$ is equal to $R 2$, the driver coil outer radius. The 


\section{6}

reason that the calculations are done over this interval is that this is the region where the defect signal is strongest, so the signal-to-noise ratio for experimental readings is highest in this region, and, therefore, the experimental readings and calculated readings agree most closely in this region.

2. Variable NZT controls the number of parts into which the defect is divided to perform the calculations. Since the theory upon which this program is based is more accurate for small defects, it is desirable to work with the defect in parts rather than as a whole. 
Integration Section of Program RFAVZSCN

\section{Symbol definitions}

The following are definitions of the symbols used to describe the program variables appearing in the integration section of this program. All lengths are normalized by dividing by the mean radius of the driver coil unless otherwise noted.

$\begin{array}{ll}\alpha & \text { Integration variable } \\ \alpha_{1} & \left(\alpha^{2}+j \omega \mu \sigma_{1} \bar{r}^{2}\right)^{1 / 2} \\ \alpha_{22} & \text { Defect shape and orientation factor } \\ \beta_{1} & \left(\alpha^{2}+j \omega \mu \sigma \bar{r}^{2}\right)^{1 / 2} / \mu \\ c & \text { Plate thickness } \\ J\left(x_{2}, x_{1}\right) & \text { Integral of } J_{1}(x) \text { with respect to } x \text { from } \alpha x_{1} \text { to } \alpha x_{2} \\ J_{1}(x) & \text { Bessel function of the first kind of order } 1 \\ l_{3} & \text { Length of driver coil } \\ l_{4} & \text { Length of pickup coil } \\ l_{5} & \text { Distance of recess of pickup coil } \\ l_{6} & \text { Lift-off of driver coil } \\ \mu & \text { Relative magnetic permeability of plate } \\ N_{3} & \text { Number of turns in the driver coil } \\ N_{4} & \text { Number of turns in each pickup coil } \\ r & \text { Coil-to-defect radial distance } \\ r & \text { Mean radius of driver coil in inches } \\ r_{1} & \text { Inner radius of driver coil } \\ r_{2} & \text { Outer radius of driver coil } \\ r_{3} & \text { Inner radius of pickup coil } \\ r_{4} & \text { Outer radius of pickup coil } \\ \sigma_{1} & \text { Conductivity of plate } \\ \text { Vol } & \text { Normalized volume of defect } \\ \omega & \text { Angular frequency at which circuit is driven } \\ z & \text { Depth to center of defect }\end{array}$

\section{Variables appearing in the integration section}

\section{Program}

variable

A1

A13

A14

A2

A2BI

A2BR

A3
Symbolic

equivalent

$\alpha \ell_{3}+\exp \left(-\alpha \ell_{3}\right)-1$

(See note II.)

$\exp \left(-\alpha \ell_{3}\right)$

$\exp \left(-\alpha \ell_{4}\right)$

$\alpha \ell_{4}+\exp \left(-\alpha \ell_{4}\right)-1$

(See note II.)

$$
\operatorname{Im}\left[\alpha^{2}-\beta_{1}{ }^{2}\right]
$$

$\operatorname{Re}\left[\alpha^{2}-\beta_{1}{ }^{2}\right]$

$1-\exp \left(-\alpha \ell_{3}\right)$ 


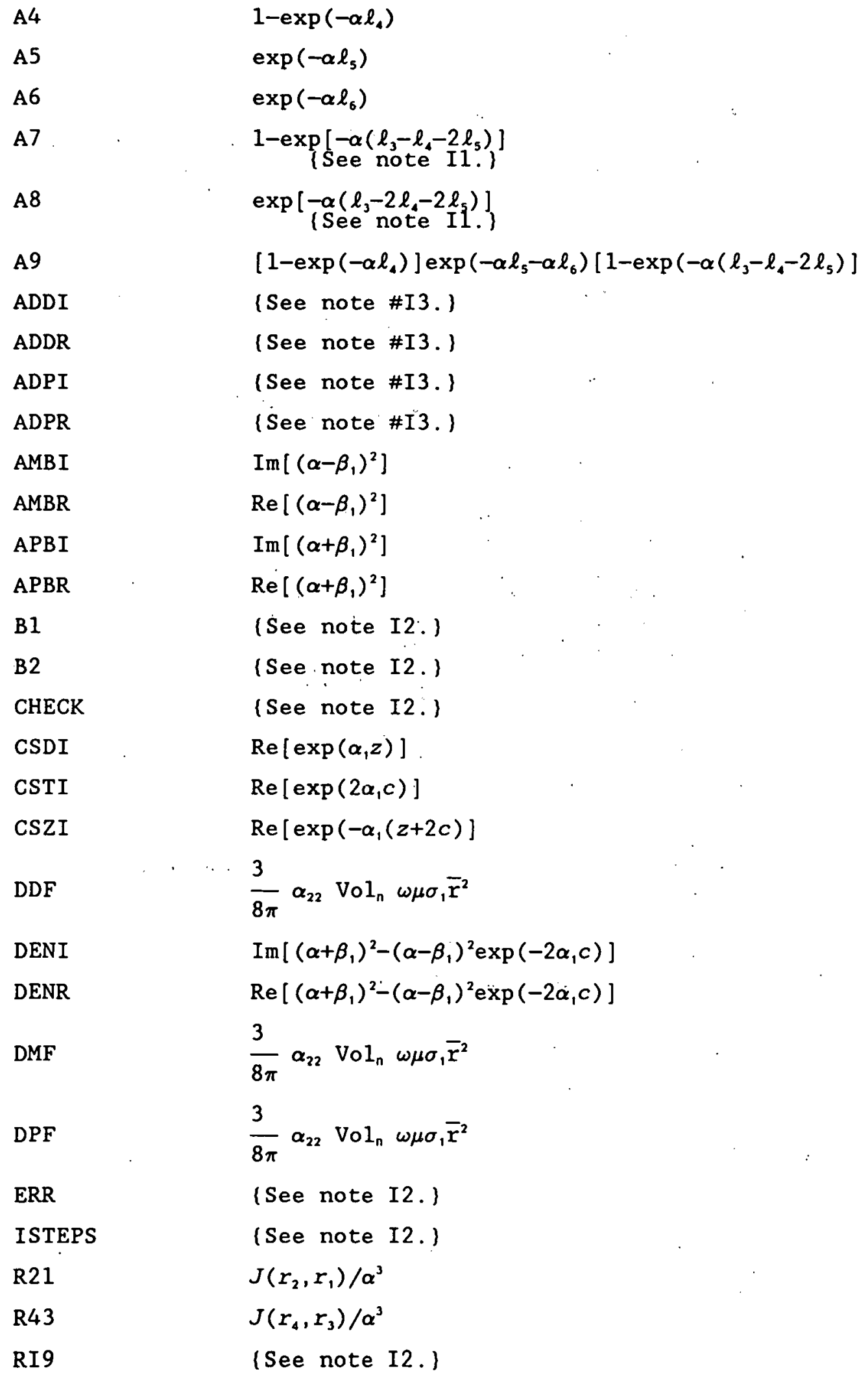


RJ 1

S1

s2

SAIR1

SAIR2

SDDR

SDPI

$\begin{aligned} & \operatorname{SDDI} {\left[\int_{0}^{\infty} \frac{1}{\alpha^{3}} J\left(r_{2}, r_{1}\right)\left(1-\exp \left(-\alpha \ell_{3}\right)\right) J_{1}(\alpha r)\left(\exp \left(-\alpha \ell_{6}\right)\right)\right.} \\ &\left.2 \alpha\left[\frac{\left(\alpha+\beta_{1}\right) \exp (\alpha, z)-\left(\alpha-\beta_{1}\right) \exp \left(-\alpha_{1}(2 c+z)\right)}{\left(\alpha+\beta_{1}\right)^{2}-\left(\alpha-\beta_{1}\right)^{2} \exp (-2 \alpha, c)}\right] \mathrm{d} \alpha\right]\end{aligned}$

$$
\mathrm{J}_{1}(\alpha r)
$$

$d \alpha$

\{See note I2.\}

$\int_{0}^{\infty} \frac{1}{\alpha^{6}}\left[J\left(r_{2}, r_{1}\right)\right]^{2} 2\left[\alpha \ell_{3}+\exp \left(-\alpha \ell_{3}\right)-1\right] \mathrm{d} \alpha$

$\int_{0}^{\infty} \frac{1}{\alpha^{6}}\left[J\left(r_{4}, r_{3}\right)\right]^{2}\left[4\left(\alpha l_{4}+\exp \left(-\alpha l_{4}\right)-1\right)+\right.$

$\left.-2\left(1-\exp \left(-\alpha \ell_{4}\right)\right)^{2} \exp \left(-\alpha\left(l_{3}-2 l_{4}-2 l_{5}\right)\right)\right] \mathrm{d} \alpha$

$\operatorname{Re}\left[\int_{0}^{\infty} \frac{1}{\alpha^{3}} J\left(r_{2}, r_{1}\right)\left(1-\exp \left(-\alpha \ell_{3}\right)\right) J_{1}(\alpha r)\left(\exp \left(-\alpha \ell_{6}\right)\right)\right.$

$\left.2 \alpha\left[\frac{\left(\alpha+\beta_{1}\right) \exp \left(\alpha_{1} z\right)-\left(\alpha-\beta_{1}\right) \exp \left(-\alpha_{1}(2 c+z)\right)}{\left(\alpha+\beta_{1}\right)^{2}-\left(\alpha-\beta_{1}\right)^{2} \exp (-2 \alpha, c)}\right] d \alpha\right]$

$\operatorname{Im}\left[\int_{0}^{\infty} \frac{1}{\alpha^{3}}\left[J\left(r_{4}, r_{3}\right)\left(1-\exp \left(-\alpha \ell_{4}\right)\right) \exp \left(-\alpha\left(l_{5}+l_{6}\right)\right)\right.\right.$

$$
\begin{aligned}
& 2 \alpha\left(1-\exp \left(-\alpha\left(\ell_{3}-\ell_{4}-2 \ell_{5}\right)\right) J_{1}(\alpha r)\right] \\
& \left.\left[\frac{\left(\alpha+\beta_{1}\right) \exp (\alpha, z)-\left(\alpha-\beta_{1}\right) \exp \left(-\alpha_{1}(2 c+z)\right)}{\left(\alpha+\beta_{1}\right)^{2}-\left(\alpha-\beta_{1}\right)^{2} \exp \left(-2 \alpha_{1} c\right)}\right] d \alpha\right]
\end{aligned}
$$


SDPR

$$
\begin{aligned}
& \operatorname{Re}\left[\int _ { 0 } ^ { \infty } \frac { 1 } { \alpha ^ { 3 } } \left[J\left(r_{4}, r_{3}\right)\left(1-\exp \left(-\alpha \ell_{4}\right)\right) \exp \left(-\alpha\left(\ell_{5}+l_{6}\right)\right)\right.\right. \\
& 2 \alpha\left(1-\exp \left(-\alpha\left(\ell_{3}-\ell_{4}-2 \ell_{5}\right)\right) J_{1}(\alpha r)\right] \\
& {\left.\left[\frac{\left(\alpha+\beta_{1}\right) \exp (\alpha, z)-\left(\alpha-\beta_{1}\right) \exp \left(-\alpha_{1}(2 c+z)\right)}{\left(\alpha+\beta_{1}\right)^{2}-\left(\alpha-\beta_{1}\right)^{2} \exp \left(-2 \alpha_{1} c\right)}\right] \mathrm{d} \alpha\right] }
\end{aligned}
$$

SFD

$$
\bar{\alpha}^{6}\left[J\left(r_{2}, r_{1}\right)\right]^{2} \mathrm{~d} \alpha
$$

SFM

$$
-_{\alpha^{6}}^{1} J\left(r_{4}, r_{3}\right) J\left(r_{2}, r_{1}\right) \mathrm{d} \alpha
$$

SFP

${\frac{1}{\alpha^{6}}}^{1}\left[J\left(r_{4}, r_{3}\right)\right]^{2} \mathrm{~d} \alpha$

SNDI

$\operatorname{Im}[\exp (\alpha, z)]$

SNTI

$\operatorname{Im}[\exp (2 \alpha, c)]$

SNZI

SZDI

$-\operatorname{Im}\left[\exp \left(-\alpha_{1}(z+2 c)\right)\right]$

$\operatorname{Im}\left[\int_{0}^{\infty} \frac{1}{\alpha^{6}}\left(1-\exp \left(-\alpha \ell_{3}\right)\right)^{2} \exp \left(-2 \alpha \ell_{6}\right)\left(J\left(r_{2}, r_{1}\right)\right)^{2}\right.$

$$
\left.\left[\frac{\left(\alpha-\beta_{1}\right)\left(\alpha+\beta_{1}\right)-\left(\alpha-\beta_{1}\right)\left(\alpha+\beta_{1}\right) \exp (-2 \alpha, c)}{\left(\alpha+\beta_{1}\right)^{2}-\left(\alpha-\beta_{1}\right)^{2} \exp \left(-2 \alpha_{1}, c\right)}\right] \mathrm{d} \alpha\right]
$$

SZDR

$$
\begin{aligned}
-\operatorname{Re} & {\left[\int_{0}^{\infty} \frac{1}{\alpha^{6}}\left(1-\exp \left(-\alpha \ell_{3}\right)\right)^{2} \exp \left(-2 \alpha \ell_{6}\right)\left(J\left(r_{2}, r_{1}\right)\right)^{2}\right.} \\
& {\left.\left[\frac{\left(\alpha-\beta_{1}\right)\left(\alpha+\beta_{1}\right)-\left(\alpha-\beta_{1}\right)\left(\alpha+\beta_{1}\right) \exp (-2 \alpha, c)}{\left(\alpha+\beta_{1}\right)^{2}-\left(\alpha-\beta_{1}\right)^{2} \exp \left(-2 \alpha_{1}, c\right)}\right] d \alpha\right] }
\end{aligned}
$$


SZMI

$\operatorname{Im}\left[\int_{0}^{\infty} \frac{1}{\alpha^{6}}\left[J\left(r_{4}, r_{3}\right) J\left(r_{2}, r_{1}\right)\left(1-\exp \left(-\alpha \ell_{3}\right)\right) \exp \left(-\alpha\left(2 \ell_{6}+\ell_{5}\right)\right)\right.\right.$

$\left(1-\exp \left(-\alpha \ell_{4}\right)\right)\left(1-\exp \left(-\alpha\left(\ell_{3}-\ell_{4}-2 \ell_{5}\right)\right]\right.$ $\left.\left[\frac{\left(\alpha-\beta_{1}\right)\left(\alpha+\beta_{1}\right)-\left(\alpha-\beta_{1}\right)\left(\alpha+\beta_{1}\right) \exp (-2 \alpha, c)}{\left(\alpha+\beta_{1}\right)^{2}-\left(\alpha-\beta_{1}\right)^{2} \exp (-2 \alpha, c)}\right] \mathrm{d} \alpha\right]$

SZMR

$-\operatorname{Re}\left[\int_{0}^{\infty} \frac{1}{\alpha^{6}}\left[J\left(r_{4}, r_{3}\right) J\left(r_{2}, r_{1}\right)\left(1-\exp \left(-\alpha l_{3}\right)\right) \exp \left(-\alpha\left(2 l_{6}+l_{5}\right)\right)\right.\right.$

$\left(1-\exp \left(-\alpha \ell_{4}\right)\right)\left(1-\exp \left(-\alpha\left(\ell_{3}-\ell_{4}-2 \ell_{5}\right)\right]\right.$

$\left.\left[\frac{\left(\alpha-\beta_{1}\right)\left(\alpha+\beta_{1}\right)-\left(\alpha-\beta_{1}\right)\left(\alpha+\beta_{1}\right) \exp (-2 \alpha, c)}{\left(\alpha+\beta_{1}\right)^{2}-\left(\alpha-\beta_{1}\right)^{2} \exp (-2 \alpha, c)}\right] \mathrm{d} \alpha\right]$

SZPI

$\operatorname{Im}\left[\int_{0}^{\infty} \frac{1}{\alpha^{6}}\left[J\left(r_{4}, r_{3}\right)\right]^{2}\left(1-\exp \left(-\alpha l_{4}\right)\right)^{2} \exp \left(-2 \alpha\left(l_{5}+l_{6}\right)\right)\right.$

$\left.\left(1-\exp \left(-\alpha\left(\ell_{3}-\ell_{4}-2 \ell_{5}\right)\right)\right)^{2}\left[\frac{\left(\alpha-\beta_{1}\right)\left(\alpha+\beta_{1}\right)-\left(\alpha-\beta_{1}\right)\left(\alpha+\beta_{1}\right) \exp (-2 \alpha, c)}{\left(\alpha+\beta_{1}\right)^{2}-\left(\alpha-\beta_{1}\right)^{2} \exp \left(-2 \alpha_{1} c\right)}\right] \mathrm{d} \alpha\right]$

SZPR

$-\operatorname{Re}\left[\int_{0}^{\infty} \frac{1}{\alpha^{6}}\left[J\left(r_{4}, r_{3}\right)\right]^{2}\left(1-\exp \left(-\alpha \ell_{4}\right)\right)^{2} \exp \left(-2 \alpha\left(\ell_{5}+\ell_{6}\right)\right)\right.$

$\left.\left(1-\exp \left(-\alpha\left(\ell_{3}-\ell_{4}-2 \ell_{5}\right)\right)\right)^{2}\left[\frac{\left(\alpha-\beta_{1}\right)\left(\alpha+\beta_{1}\right)-\left(\alpha-\beta_{1}\right)\left(\alpha+\beta_{1}\right) \exp (-2 \alpha, c)}{\left(\alpha+\beta_{1}\right)^{2}-\left(\alpha-\beta_{1}\right)^{2} \exp \left(-2 \alpha_{1} c\right)}\right] \mathrm{d} \alpha\right]$

TI

$\operatorname{IIII}\left[2 u_{1} c\right]$

TR

$\operatorname{Re}[2 \alpha, c]$

TZI

$\operatorname{Im}\left[\alpha_{1}(2 c+z)\right]$

TZR

$\operatorname{Re}\left[\alpha_{1}(2 c+z)\right]$

$\mathrm{X}$

$\alpha$

$\mathrm{X} 1$

$\operatorname{Re}\left(\beta_{1}\right)$ 
$\mathrm{X} 1 \mathrm{X}$

XJR1

XJR2

XJR3

XJR4

XL3

XL4

XL7

XL 8

XPDR

XPTR

XPZR

XRD

$\mathrm{XX}$

$\mathrm{XX1}$

$\mathrm{XY} 1$

Y1

ZDF

ZDI

ZDIM

ZDR

ZDRL

ZIM

ZMF

ZNDI

ZNDR

ZNUI

ZNUR
$\operatorname{Re}\left[\alpha\left(\beta_{1}-\alpha\right)\right]$

$J\left(r_{1}, 0\right) / \alpha^{3}$

$J\left(r_{2}, 0\right) / \alpha^{3}$

$J\left(r_{3}, 0\right) / \alpha^{3}$

$J\left(r_{4}, 0\right) / \alpha^{3}$

$\alpha \ell_{3}$

$\alpha \ell_{4}$

$\alpha\left(l_{3}-\ell_{4}-2 \ell_{5}\right)$

$\alpha\left(l_{3}-2 l_{4}-2 l_{5}\right)$

$\exp [\operatorname{Re}(\alpha, z)]$

$\exp [\operatorname{Re}(2 \alpha, c)]$

$\exp \left[\operatorname{Re}\left(-\alpha_{1}(2 c+z)\right)\right]$

$\alpha r$

$\alpha^{2}$

$\operatorname{Re}\left[\alpha\left(\beta_{1}+\alpha\right)\right]$

$\operatorname{Im}[\alpha(\beta,+\alpha)]=\operatorname{Im}[\alpha(\beta,-\alpha)]$

$\operatorname{Im}\left(\beta_{1}\right)$

$\frac{\omega \pi \mu_{0} N_{3}^{2}}{\left(r_{2}-r_{1}\right)^{2} \ell_{3}^{2}}$

$\operatorname{Im}(\alpha, z)$

$\operatorname{Im}\left[\alpha \frac{\left(\alpha+\beta_{1}\right) \exp (\alpha, z)-\left(\alpha-\beta_{1}\right) \exp \left(-\alpha_{1}(2 c+z)\right)}{\left(\alpha+\beta_{1}\right)^{2}-\left(\alpha-\beta_{1}\right)^{2} \exp \left(-2 \alpha_{1} c\right)}\right]$

$\operatorname{Re}(\alpha, z)$

$\operatorname{Re}\left[\alpha \frac{\left(\alpha+\beta_{1}\right) \exp \left(\alpha_{1} z\right)-\left(\alpha-\beta_{1}\right) \exp \left(-\alpha_{1}(2 c+z)\right)}{\left(\alpha+\beta_{1}\right)^{2}-\left(\alpha-\beta_{1}\right)^{2} \exp \left(-2 \alpha_{1} c\right)}\right]$

$\operatorname{Im}\left[\frac{\left(\alpha-\beta_{1}\right)\left(\alpha+\beta_{1}\right)-\left(\alpha-\beta_{1}\right)\left(\alpha+\beta_{1}\right) \exp \left(-2 \alpha_{1} c\right)}{\left(\alpha+\beta_{1}\right)^{2}-\left(\alpha-\beta_{1}\right)^{2} \exp \left(-2 \alpha_{1} c\right)}\right]$

$\frac{\omega \pi \mu_{0} N_{3} N_{4}}{\left(r_{2}-r_{1}\right)\left(r_{4}-r_{3}\right) \ell_{3} \ell_{4}}$

$\operatorname{Im}\left[\alpha\left(\beta_{1}+\alpha\right) \exp \left(\alpha_{1} z\right)+\alpha\left(\beta_{1}-\alpha\right) \exp \left(-\alpha_{1}(2 c+z)\right)\right]$

$\operatorname{Re}\left[\alpha\left(\beta_{1}+\alpha\right) \exp (\alpha, z)+\alpha(\beta,-\alpha) \exp \left(-\alpha_{1}(2 c+z)\right)\right]$

$\operatorname{Im}\left[\left(\alpha^{2}-\beta_{1}{ }^{2}\right)-\left(\alpha^{2}-\beta_{1}{ }^{2}\right) \exp (-2 \alpha, c)\right]$

$\operatorname{Re}\left[\left(\alpha^{2}-\beta_{1}{ }^{2}\right)-\left(\alpha^{2}-\beta_{1}{ }^{2}\right) \exp (-2 \alpha, c)\right]$ 


$$
\begin{array}{ll}
\text { ZPF } & \frac{\omega \pi \mu_{0} N_{4}^{2}}{\left(r_{4}-r_{3}\right)^{2} \ell_{4}^{2}} \\
\text { ZRL } & \operatorname{Re}\left[\frac{\left(\alpha-\beta_{1}\right)\left(\alpha+\beta_{1}\right)-\left(\alpha-\beta_{1}\right)\left(\alpha+\beta_{4}\right) \exp \left(-2 \alpha_{1} c\right)}{\left(\alpha+\beta_{1}\right)^{2}-\left(\alpha-\beta_{1}\right)^{2} \exp \left(-2 \alpha_{1} c\right)}\right]
\end{array}
$$

Notes for the integration section

I1. A number of the variables in the integration section are not always assigned their exact values but are approximated in certain cases to save time. For example, rather than calculate: the: exponential of a very small number, the Maclaurin series expansion is sometimes used. Also, the exponential of a very large negative number is usually treated as zero.

I2. Several variables appear in the integration section of the program which play no part in the calculations being, done. They are merely there to do such things as to determine the maximum step size which can be used while still accurately calculating: the integrals.

I3. Variables ADDR, ADDI, ADPR, and ADPI are the averages of the elements in arrays SDDR, SDDI, SDPR, and SDPI, respectively. For improved accuracy, the defect is divided into NZT parts to perform the calculations. The NZT elements of each array: contain the calculations for these NZT parts. These elements are averaged: to give the total effect of all of these parts on the impedances of: the coils.

\section{Sample output}

Printer output of program RFAVZSCN:

RFAVZSCN 20 POINTS USED TIME $9: 42: 19$ DATE $8 / 9 / 89$

PROBE 250A PLATE THIK 0.2500

COIL IN RAD OT RAD LENGTH OLO/REC TURNS COIL RES CKT: RES CAP

$\begin{array}{llllllllllll}\text { DRIVER } & 0.7500 & 1.2500 & 0.6000 & 0.0600 & 2350.0 & 5.310 \mathrm{E}+02 & 3.050 \mathrm{E}+03 & 1.500 \mathrm{E}-10\end{array}$

$\begin{array}{lllllllll}\text { PICKUP } & 0.3500 & 0.7000 & 0.2000 & 0.0000 & 3450.0 & 5.147 \mathrm{E}+03 & 1.000 \mathrm{E}+06 & 1.500 \mathrm{E}-10\end{array}$

RBAR 0.2500 FREQ- $5.000000 E+02 \mathrm{RHO}=4.0540$ PERM $=1.000$ WUSRR $=3.9267$

NEAR SIDE DEFECT: DIAM $=0.2215$, DEPTH $=0.2215$

GAIN 1. DVR VOLT 1.1000 NOR DEF VOL 5.4625E-01 DVR AIR IND 6.734812E-02

Partial listing of file FORT39:

$\begin{array}{lll}0.359 & 0.212 D-03 & 125.01 \\ 0.368 & 0.221 D-03 & 125.06 \\ 0.377 & 0.230 D-03 & 125.11 \\ 0.386 & 0.239 D-03 & 125.15 \\ 0.395 & 0.248 D-03 & 125.19 \\ 0.404 & 0.256 D-03 & 125.23 \\ 0.413 & 0.265 D-03 & 125.26\end{array}$


$\begin{array}{lll}0.422 & 0.273 \mathrm{D}-03 & 125.28\end{array}$

$\begin{array}{llll}0.431 & 0.282 \mathrm{D}-03 & 125.30\end{array}$

$\begin{array}{llll}0.440 & 0.290 \mathrm{D}-03 & 125.31\end{array}$ 


\section{Listing}

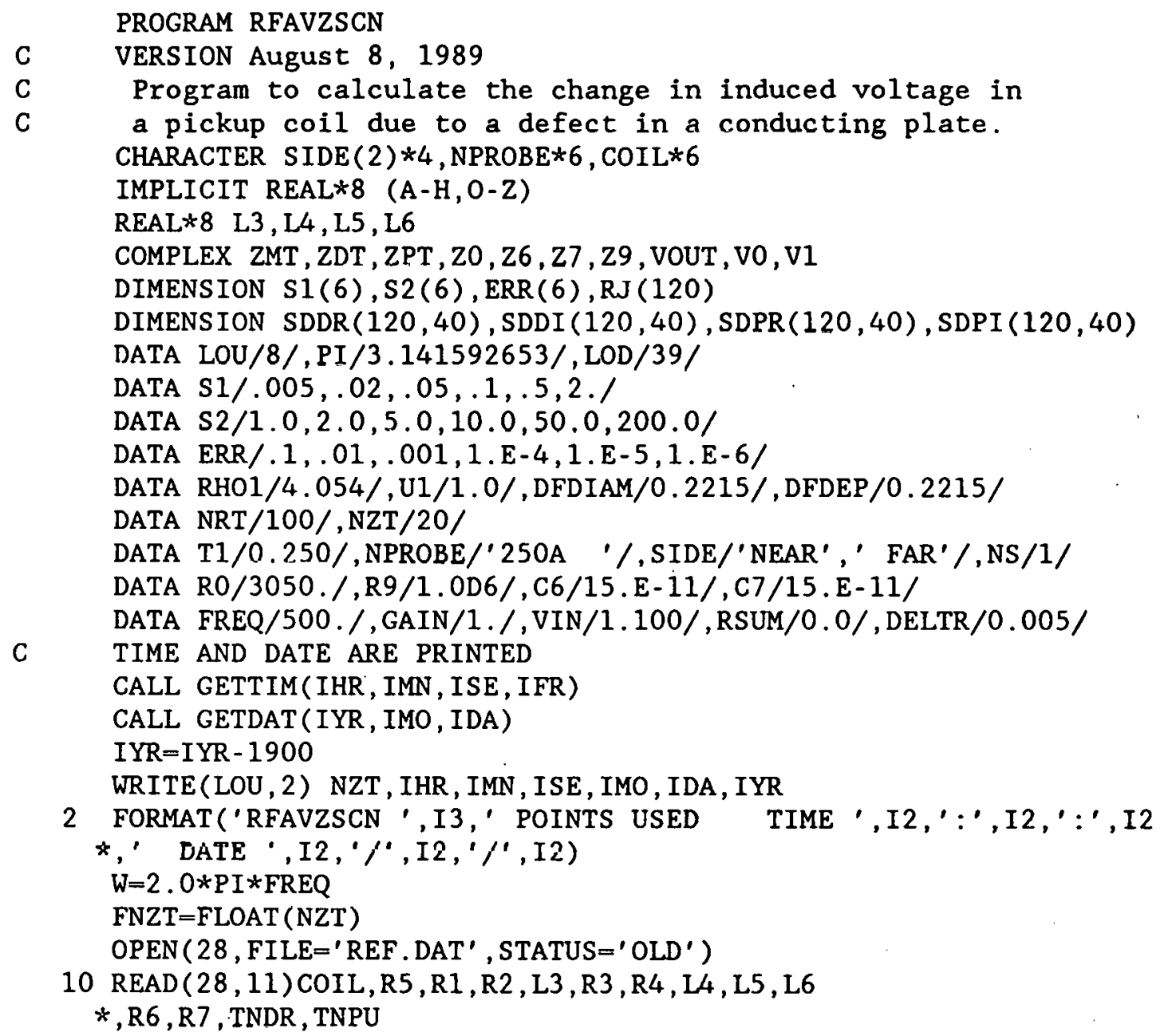

11 FORMAT $(A 6,9 \mathrm{~F} 8.4, \mathrm{~F} 10.4, \mathrm{~F} 11.4,2 \mathrm{~F} 8.1)$

IF(COIL.EQ. 'END ')WRITE $(0, *)$ ' COIL NOT FOUND'

TF(COTL..EQ.' END ') GO TO 1020

IF (COIL. NE. NPROBE) GO TO 10

$\mathrm{L} 6=\mathrm{L} 6+0.010 / \mathrm{R} 5$

WRITE (LOU, 3) NPROBE, T1

3 FORMAT('PROBE ',A6,' PLATE THIK',F7.4)

WRITE (LOU, 5)

5 FORMAT('COIL IN RAD OT RAD LENGTH OLO/REC TURNS',

*' COIL RES CKT: RES CAP')

WRITE (LOU , 14) R1 , R2 , L3 , L6 , TNDR , R6 , R0, C6

WR ITE (LOU, 15)R3 , R4, L4 , L5, TNPU, R7, R9, C7

14 FORMAT('DRIVER ' , 4(F7.4,1X), F8.1,3(1PE10.3))

15 FORMAT('PICKUP ', 4(F7.4,1X), F8.1,3(1PE10.3))

WUSRR $=0.5093979 * \mathrm{U} 1 * \mathrm{R} 5 * \mathrm{R} 5 * \mathrm{FREQ} / \mathrm{RHO} 1$

WRITE (LOU , 20)R5, FREQ, RHO1 , U1, WUSRR

20 FORMAT ('RBAR' ,F7.4,' FREQ= ', 1PE13.6,' RHO-', OPF9.4, *' PERM=',F7.3,' WUSRR=' ,F9.4) 
C WRITE(LOD , 23) NPROBE, T1, FREQ, SIDE(NS), DFDIAM, DFDEP WRITE $(0,23)$ NPROBE , T1 , FREQ , SIDE (NS), DFDIAM, DFDEP

23 FORMAT('PROBE ', A6,' PLATE THK', F7.4,' FREQ=', 1PE8 .1,1X, A4, *' SIDE' ,OPF6.4,' DIA', F6.4,' DEEP')

DELTR= $=(R 2-R 3) / N R T$

$\mathrm{RD}=\mathrm{R} 3$

$\mathrm{ZD}=-\mathrm{DFDEP}$

$\mathrm{TD}=\mathrm{TNDR} /((\mathrm{R} 2-\mathrm{R} 1) * \mathrm{~L} 3)$

$\mathrm{TP}=\mathrm{TNPU} /((\mathrm{R} 4-\mathrm{R} 3) * \mathrm{~L} 4)$

$\mathrm{ZDF}=6.300475 \mathrm{E}-7 * \mathrm{FREQ} * \mathrm{TD} * \mathrm{TD} * \mathrm{R} 5$

$\mathrm{ZPF}=6.300475 \mathrm{E}-7 * \mathrm{FREQ} * \mathrm{TP} * \mathrm{TP} * \mathrm{R} 5$

$\mathrm{ZMF}=6.300475 E-7 * \mathrm{FREQ} * \mathrm{TD} * \mathrm{TP} * \mathrm{R} 5$

C

VOLN $=0.1666667 * \mathrm{PI} *($ DFDIAM $($ NRUN $) / R 5) *($ DFDIAM $($ NRUN $) / R 5) *($ DFDIAM $/ R 5)$

VOLN $=$ PI $*$ DFDIAM $*$ DFDIAM*DFDEP $/(4 . * R 5 * R 5 * R 5)$

$\mathrm{DDF}=0.1193662 *$ VOLN $*$ WUSRR

$D P F=0.1193662 *$ VOLN*WUSRR

DMF $=0.1193662 *$ VOLN $*$ WUSRR

WRITE (LOU, 18) SIDE (NS), DFDIAM, DFDEP

18 FORMAT(A4," SIDE DEFECT: DIAM=',F7.4,', DEPTH=',F7:4)

$\mathrm{ZD}=-\mathrm{DFDEP} / \mathrm{R} 5$

$\mathrm{T} 1=\mathrm{T} 1 / \mathrm{R} 5$

SAIR $1=0.0$

SAIR2 $=0.0$

$S Z D R=0.0$

$S Z D I=0.0$

$S Z R R=0.0$

$S Z P I=0.0$

$S Z M R=0.0$

$S Z M I=0.0$

DO $27 \mathrm{NR}=1, \mathrm{NRT}$

DO $25 \mathrm{NZ}=1, \mathrm{NZT}$

$\operatorname{SDDR}(\mathrm{NR}, \mathrm{NZ})=0.0$

$\operatorname{SDDI}(\mathbb{N R}, \mathbb{N Z})=0.0$

$\operatorname{SDPR}((\mathrm{NR}, \mathrm{NZ})=0.0$

$25 \operatorname{SDPI}(\mathrm{NR}, \mathrm{NZ})=0.0$

27 CONTINUE

$B .1=0.0$

$\mathbb{B} 2=S 2$ (1)

DO 100 JKL $=1,6$

30 RI $9=$ SAIR 1

$\mathbb{X}=\mathrm{B} 1-0.5 * \mathrm{~S} 1(\mathrm{~J} \mathrm{KL})$

(C) DETERMINE NUMBER OF STEPS, ISTEPS, BASED ON STEP SIZE\&INTERVAL

ISTEPS $=$ DNINT $((\mathrm{B} 2-\mathrm{B} 1) / \mathrm{S} 1(\mathrm{JKL}))$

DO $95 \mathrm{I}=1$, ISTEPS

$X=\mathbb{X}+S I(J K L)$

CALL BESSEL(XJR2, $X, R 2$ )

CALL BESSEL(XJR1, X, R1)

CALL BESSEL(XJR4, $X, R 4)$

CALL BESSEL(XJR3, $X, R 3)$

$\mathbb{R} 21=\mathrm{XJR} 2-\mathrm{XJR} 1$

$\mathbb{R} 43=\mathrm{XJR} 4 \mathrm{XJJ} 3$ 
$\mathrm{XL} 3=\mathrm{X} * \mathrm{~L} 3$

IF (XL3.GT.5.0E-3) GO TO 60

$\mathrm{A} 1=\mathrm{XL} 3 * \mathrm{XL} 3 *(0.5-\mathrm{XL} 3 / 6.0)$

GO TO 80

60 IF(XL3.GT.75.0) GO TO 70

$A 1=X L 3+D E X P(-X L 3)-1.0$

GO TO 80

$70 \cdot \mathrm{Al}=\mathrm{XL} 3-1.0$

$80 \mathrm{~A} 3=\mathrm{XL} 3-\mathrm{Al}$

$\mathrm{SFD}=\mathrm{S} 1(\mathrm{JKL}) * \mathrm{R} 21 * \mathrm{R} 21$

$S F P=S 1(J K L) * R 43 * R 43$

$S F M=S 1(J K L) * R 21 * R 43$

SAIR $1=S A I R 1+S F D * 2.0 * A 1$

$\mathrm{XL} 4=\mathrm{X} * \mathrm{~L} 4$

IF (XL4.GT.5.0E-3) GO TO 81

$\mathrm{A} 2=\mathrm{XL} 4 * \mathrm{XL} 4 *(0.5-\mathrm{XL} 4 / 6.0)$

GO TO 83

81 IF(XL4.GT.75.0) GO TO 82

$\mathrm{A} 2=\mathrm{XL} 4+\mathrm{DEXP}(-\mathrm{XL} 4)-1.0$

GO TO 83

82 A2 $=X L 4-1.0$

$83 \mathrm{~A} 4=\mathrm{XL} 4-\mathrm{A} 2$

$\mathrm{A} 13=1.0-\mathrm{A} 3$

A14 $=1.0-A 4$

$\mathrm{A} 5=\mathrm{DEXP}(-\mathrm{X} * \mathrm{~L} 5)$

$\mathrm{XL} 8=\mathrm{X} *(\mathrm{~L} 3-2 . * \mathrm{~L} 4-2, * \mathrm{~L} 5)$

IF (XL8.GT.75.) A8 $=0$.

IF (XL8, LE , 75, ) A $8=\mathrm{DEXP}(-\mathrm{XL} 8)$

SAIR2 $=$ SAIR2+SFP* $(4.0 * A 2-2.0 * A 4 * A 4 * A 8)$

IF $(X *$ L6.GT.75.) GO TO 95

C

CALCULATION OF GAMMA FACTORS

$\mathrm{XX}=\mathrm{X} * \mathrm{X}$

$\mathrm{X} 1=\mathrm{DSQRT}(0.5 *(\mathrm{XX}+\mathrm{DSQRT}(\mathrm{XX} * \mathrm{XX}+\mathrm{WUSRR} * \mathrm{WUSRR}))) / \mathrm{U} 1$

$\mathrm{Y} 1=W U S R R /(2 * * \mathrm{X} 1 * \mathrm{U} 1 * \mathrm{U} 1)$

$\mathrm{A} 6=\mathrm{DEXP}(-\mathrm{X} * \mathrm{~L} 6)$

$\mathrm{XL} 7=\mathrm{X} *(\mathrm{~L} 3-\mathrm{L} 4-2 . * \mathrm{~L} 5)$

$\mathrm{IF}(\mathrm{XL7} . \mathrm{GT} .75.) \mathrm{A} 7=1.0$

IF (XL7.LE . 75.)A7=1.0-DEXP (-XL7)

$A 9=A 4 * A 5 * A 6 * A 7$

$\mathrm{APBR}=(\mathrm{X}+\mathrm{X} 1) *(\mathrm{X}+\mathrm{X} 1)-\mathrm{Y} 1 * \mathrm{Y} 1$

$\mathrm{APBI}=2 . * \mathrm{Y} 1 *(\mathrm{X}+\mathrm{XI})$

$\mathrm{AMBR}=(\mathrm{X}-\mathrm{X} 1) *(\mathrm{X}-\mathrm{X} 1)-\mathrm{Y} 1 * \mathrm{Y} 1$

AMBI $=-2 \cdot * \mathrm{Y} 1 *(\mathrm{X}-\mathrm{X} 1)$

$\mathrm{A} 2 \mathrm{BR}=0.0$

$\mathrm{A} 2 \mathrm{BI}=-2 . * \mathrm{X} 1 * \mathrm{Y} 1$

$\mathrm{ZNUK}=\mathrm{A} 2 \mathrm{BR}$

$\mathrm{ZNUI}=\mathrm{A} 2 \mathrm{BI}$

$\mathrm{DENR}=\mathrm{APBR}$

$\mathrm{DENI}=\mathrm{APBI}$

$D N C J=D E N R * D E N R+D E N I * D E N I$

C

SECTION THAT MULTIPLIES BY DEXP(ALPHA $*$ ZDEFECT) 
DO $91 \mathrm{NZ}=1$, NZT

C NEAR SIDE DEFECT CALCULATION

FZD $=($ FLOAT $(N Z)-.5) * Z D / F N Z T$

C FAR SIDE DEFECT CALCULATION

IF (NS . EQ . 2) FZD $=-T 1-F Z D$

$\mathrm{ZDR}=\mathrm{XI} * \mathrm{UI} * \mathrm{FZD}$

IF(ZDR.LT. - 60.0)GO TO 93

$\mathrm{ZDI}=\mathrm{YI} * \mathrm{U} 1 * \mathrm{FZD}$

$X P D R=D E X P(Z D R)$

$\operatorname{CSDI}=\mathrm{DCOS}(\mathrm{ZDI}) * \mathrm{XPDR}$

$\operatorname{SNDI}=\operatorname{DSIN}(Z D I) * X P D R$

$\mathrm{XXI}=\mathrm{X} * \mathrm{XI}+\mathrm{XX}$

$\mathrm{XY} 1=\mathrm{X} * \mathrm{Y} 1$

$\mathrm{X} 1 \mathrm{X}=\mathrm{X} * \mathrm{X} 1-\mathrm{XX}$

$\mathrm{ZNDR}=\mathrm{XXI} 1 * \mathrm{CSDI}-\mathrm{XY} 1 * \mathrm{SNDI}$

$\mathrm{ZNDI}=\mathrm{XX1} * \mathrm{SNDI}+\mathrm{XY} 1 * \mathrm{CSDI}$

C SECTION THAT MULTIPLIES BY DEXP(ALPHA $1 *(2 *$ TH $+Z D E F E C T))$

$\mathrm{TZR}=\mathrm{X} 1 * \mathrm{U} 1 *(2 . * \mathrm{~T} 1+\mathrm{FZD})$

IF(TZR.GT.60.) GO TO 87

$\mathrm{TZI}=\mathrm{Y} 1 * \mathrm{U} 1 *(2, * \mathrm{~T} 1+\mathrm{FZD})$

$\mathrm{XPZR}=\mathrm{DEXP}(-\mathrm{TZR})$

CSZI $=$ DCOS $(T Z I) * X P Z R$

SNZI $=$ DSIN $(T Z I) * X P Z R$

$\mathrm{ZNDR}=\mathrm{XXI} * \mathrm{CSDI}-\mathrm{XY} 1 * \mathrm{SNDI}+\mathrm{X} 1 \mathrm{X} * \mathrm{CSZI}+\mathrm{XY} 1 * \mathrm{SNZZ}$

$\mathrm{ZNDI}=\mathrm{XXI} * \mathrm{SNDI}+\mathrm{XY} 1 * \mathrm{CSDI}+\mathrm{XYI} * \mathrm{CSZI}-\mathrm{XIX} * \mathrm{SNZI}$

C. SECTION THAT MULTIPLIES BY DEXP (-ALPHA $1 * 2 *$ CLADTH)

$\mathrm{TR}=2 . * \mathrm{X} 1 * \mathrm{U} 1 * \mathrm{~T} 1$

IF (TR..GT -60, ) GO TO 87

$\mathrm{TI}=2 . * \mathrm{Y} I * \mathrm{U} 1 * \mathrm{~T} 1$

XPTR=DEXP $(-\mathrm{TR})$

CSTI $=$ DCOS $($ TI $) * X P T R$

$\mathrm{SNTI}=\mathrm{DSIN}(\mathrm{TI}) * \mathrm{XPTR}$

$D E N R=A P B R-A M B R * C S T I-A M B I * S N T I$

DENII $=A P B I-A M B I * C S T I+A M B R * S N T I$

$\mathrm{ZNEUR}=\mathrm{A} 2 \mathrm{~B} \mathrm{BR}-\mathrm{A} 2 \mathrm{BR} * \mathrm{CSTI}-\mathrm{A} 2 \mathrm{BI} * \mathrm{SNTI}$

$\mathrm{ZNUII}=\mathrm{A} 2 \mathrm{BI}-\mathrm{A} 2 \mathrm{BI} * \mathrm{CSTI}+\mathrm{A} 2 \mathrm{BR} * \mathrm{SNTI}$

DNCII $=$ DENR $*$ DENR + DENI $*$ DENI

877 ZBRL $=(Z N D R * D E N R+Z N D I * D E N I) / D N C J$

$\mathrm{ZBIM}=(\mathrm{DENR} * \mathrm{ZNDI}-\mathrm{ZNDR} * \mathrm{DENI}) / \mathrm{DNCJ}$

C LOOP OVER THE R VARIATION FOR THE DEFECT

DO $90 \mathrm{NR}=1, \mathrm{NRT}$

IF'(NZ.GT.1) GO TO 89

$R: D=R ' 3+D E L T R * F L O A T(N R)$.

$\mathrm{XRD}=\mathrm{X} * \mathrm{RD}$

CALL BESEL1 (XRD, RJ 1 )

$\mathrm{RJ}(\mathrm{NR})=\mathrm{RJ} 1$

C:

SUM DEFECT SENSITIVITY FACTORS FOR THE DRIVER \& PICKUP COILS

899) $\operatorname{SDDR}(\mathrm{NR}, \mathrm{NZ})=\mathrm{SDDR}(\mathrm{NR}, \mathrm{NZ})+\mathrm{A} 3 * \mathrm{~A} 6 * \mathrm{RJ}(\mathrm{NR}) * 2 * \mathrm{ZDRL} * \mathrm{R} 21, * \mathrm{~S} 1$ (JKL)

$\mathrm{SDDII}(\mathrm{NR}, \mathrm{NZ})=\mathrm{SDDI}(\mathrm{NR}, \mathrm{NZ})+\mathrm{A} 3 * \mathrm{~A} 6 * \mathrm{RJ}(\mathrm{NR}) * 2 * \mathrm{ZDIM} * \mathrm{R} 21 * \mathrm{~S} 1(\mathrm{JKL})$

$\mathrm{SBPR}(\mathrm{NR}, \mathrm{NZ})=\mathrm{SDPR}(\mathrm{NR}, \mathrm{NZ})+\mathrm{A} 9 * \mathrm{RJ}(\mathrm{NR}) * 2 * \mathrm{ZDRL} * \mathrm{R} 43 * \mathrm{~S} 1(\mathrm{JKL})$

$90 \operatorname{SDPI}(\mathrm{NR}, \mathrm{NZ})=\mathrm{SDPI}(\mathrm{NR}, \mathrm{NZ})+\mathrm{A} 9 * \mathrm{RJ}(\mathrm{NR}) * 2 * \mathrm{ZDIM} * \mathrm{R} 43 * \mathrm{~S} 1(\mathrm{JKL})$ 
91 CONTINUE

$93 \mathrm{ZRL}=(\mathrm{ZNUR} * \mathrm{DENR}+\mathrm{ZNUI} * \mathrm{DENI}) / \mathrm{DNCJ}$

$Z I M=(D E N K * Z N U I-Z N U R * D E N I) / D N C J$

SZDI $=S Z D I+A 3 * A 3 * A 6 * A 6 * Z R L * S F D$

$S Z D R=S Z D R-A 3 * A 3 * A 6 * A 6 * Z I M * S F D$

$S Z P I=S Z P I+A 9 * A 9 * Z R L * S F P$

$S Z P R=S Z P R-A 9 * A 9 * Z I M * S F P$

$S Z M I=S Z M I+A 3 * A 6 * A 9 * Z R L * S F M$

$S Z M R=S Z M R-A 3 * A 6 * A 9 * Z I M * S F M$

95 CONTINUE

$\mathrm{B} 1=\mathrm{B} 2$

$\mathrm{B} 2=\mathrm{B} 2+\mathrm{S} 2(\mathrm{JKL})$

CHECK $=($ SAIR1 - RI9) $/$ SAIR1

IF (ABS (CHECK).GT.ERR (JKL)) GO TO 30

100 CONTINUE

C

COMPUTATION OF DRIVER INDUCTANCE

$\mathrm{Q} 6=\mathrm{ZDF} * \operatorname{SAIR} 1 / \mathrm{W}$

WRITE (LOU, 120) GAIN, VIN, VOLN, Q6

120 FORMAT ('GAIN' , F10.0,' DVR VOLT', F7.4,' NOR DEF. VOL', 1PE11.4, *' DVR AIR IND', 1PE13.6)

C DEFINE COMPLEX QUANTITIES THAT ARE CONSTANT

ZO $=\operatorname{DCMPLX}(0.0 \mathrm{DO},-\mathrm{RO})$

$\mathrm{Z} 6=\mathrm{DCMPLX}(\mathrm{W} * \mathrm{C} 6 * \mathrm{R} 0,-1.0 \mathrm{D} 0)$

$\mathrm{Z7}=\mathrm{DCMPLX}(\mathrm{W} * \mathrm{C} 7 * \mathrm{R} 9,-1 . \mathrm{OD} 0)$

$\mathrm{Z9}=\mathrm{DCMPLX}(0.0 \mathrm{DO},-\mathrm{R} 9)$

C SCAN PAST THE DEFECT IN THE R DIRECTION

DO 1010 NR=1, NRT

$\mathrm{RD}=\mathrm{R} 3+\mathrm{DELTR} * \mathrm{FLOAT}(\mathrm{NR})$

$\mathrm{D}=0$.

C AVERAGE DEFF.CT VALUES OVER DEPTH

$\mathrm{ADDR}=0.0$

$A D D I=0.0$

$\mathrm{ADPR}=0.0$

$\mathrm{ADPI}=0.0$

DO $125 \mathrm{NZ}=1$, NZT

$\mathrm{ADDR}=\mathrm{ADDR}+\mathrm{SDDR}(\mathrm{NR}, \mathrm{NZ}) / \mathrm{FNZT}$

$A \bar{D} I=A D D I+S D D I(N R, N Z) / F N Z T$

$A D P R=A D P R+S D P R(N R, N Z) / F N Z T$

125 ADPI $=A D P I+S D P I(N R, N Z) / F N Z T$

$135 \mathrm{ZDTR}=\mathrm{ZDF} *(\mathrm{SZDR}-\mathrm{D} * \mathrm{DDF} *(\mathrm{ADDR} * \mathrm{ADDR}-\mathrm{ADDI} * \mathrm{ADDI}))$

$Z D T I=Z D F *(S A I R 1+S Z D I-D * D D F * 2 . * A D D R * A D D I)$

$Z P T R=Z P F *(S Z P R-D * D P F *(A D P R * A D P R-A D P I * A D P I))$

$Z P T I=Z P F *(S A I R 2+S Z P I-D * D P F * 2 . * A D P R * A D P I)$

$\mathrm{ZMTR}=\mathrm{ZMF} *(\mathrm{SZMR}-\mathrm{D} * \mathrm{DMF} *(\mathrm{ADDR} * \mathrm{ADPR}-\mathrm{ADDI} * \mathrm{ADPI}))$

$Z M T I=Z M F *(S Z M I-D * D M F *(A D D I * A D P R+A D D R * A D P I))$

C DEHINE CUMPLEX QUANTIIIES, DO COMPLEX CIRCUIT CALCULATIONS

ZDT=DCMPLX (ZDTR, ZDTI)

ZPT $=$ DCMPLX (ZPTR, ZPTI $)$

$Z M T=D C M P L X(Z M T R, Z M T I)$

VOUT $=(-Z M T * V I N * G A I N * R 9) /$

$*(\mathrm{Z} 6 * \mathrm{Z} 7 * \mathrm{ZMT} * \mathrm{ZMT}+(\mathrm{Z} 6 *(\mathrm{ZDT}+\mathrm{R} 6)+\mathrm{Z} 0) *(\mathrm{Z} 7 *(\mathrm{ZPT}+\mathrm{R} 7)+\mathrm{Z9}))$ 
IF (D. EQ . 0) VO=VOUT

IF (D. EQ . 1) V1=VOUT

IF(D.EQ.1.) GO TO 1000

C REPEAT CALCULATIONS WITH DEFECT

$D=1.0$

GO TO 135

1000 CONTINUE

VVOR=REAL $(\mathrm{VO})$

$\mathrm{VVOI}=\mathrm{AIMAG}(\mathrm{VO})$

VVIR=REAL (V1)

VVII=AIMAG (V1)

DVVR $=$ VV1R - VVOR

DVVI $=$ VV1I - VVOI

$T T S R=T T S R-D V V R * D E L T R$

TTS I $=$ TTS I - DVVI $*$ DELTR

TMAG $=$ DSQRT $(D V V R * D V V R+D V V I * D V V I)$

$\mathrm{PHA}=($ ATAN2 (DVVI, DVVR) $) * 180 . / \mathrm{PI}$

C $\quad \operatorname{WRITE}(0,180) \mathrm{RD}, \mathrm{TMAG}, \mathrm{PHA}$

WRITE (LOD , 180) RD, TMAG , PHA

180 FORMAT (F6. 3, 1X, D11 . 3, 1X, F7 . 2)

1010 CONTINUE

C WRITE (LOU, *) TTSR, TTSI

TTMAG $=\mathrm{DSQRT}(\mathrm{TTSR} *$ TTSR+TTS I $*$ TTSI $)$

TTPHA=DATAN2 (TTS I , TTSR $) * 180 . /$ PI

C WRITE(LOU, *)'MAG ', TTMAG

C WRITE(LOU, *) ' PHA ', TTPHA

1020 STOP 'JOB' '

END 
RFGRAPH plots two sets of data on same graph

Program RFGRAPH plots two sets of data on the same graph and sends the output to the screen and to the printer. It is normally used to compare the calculated and experimental change in the induced voltage in a pickup coil due to a defect as the coil is scanned past the defect.

\section{Summary}

1. Dimension arrays and declare variable types.

2. Initialize variables.

3. Open the data files containing the input data.

4. Read in and scale the data.

5. Plot the data to the screen.

6. Send the contents of the screen to the printer.

\section{Variables}

$\mathrm{C} 6^{\circ}$

$\mathrm{C7}^{\circ}$

CGIM

The total shunt capacitance in farads of the driving circuit.

The total shunt capacitance in farads of the pickup circuit.

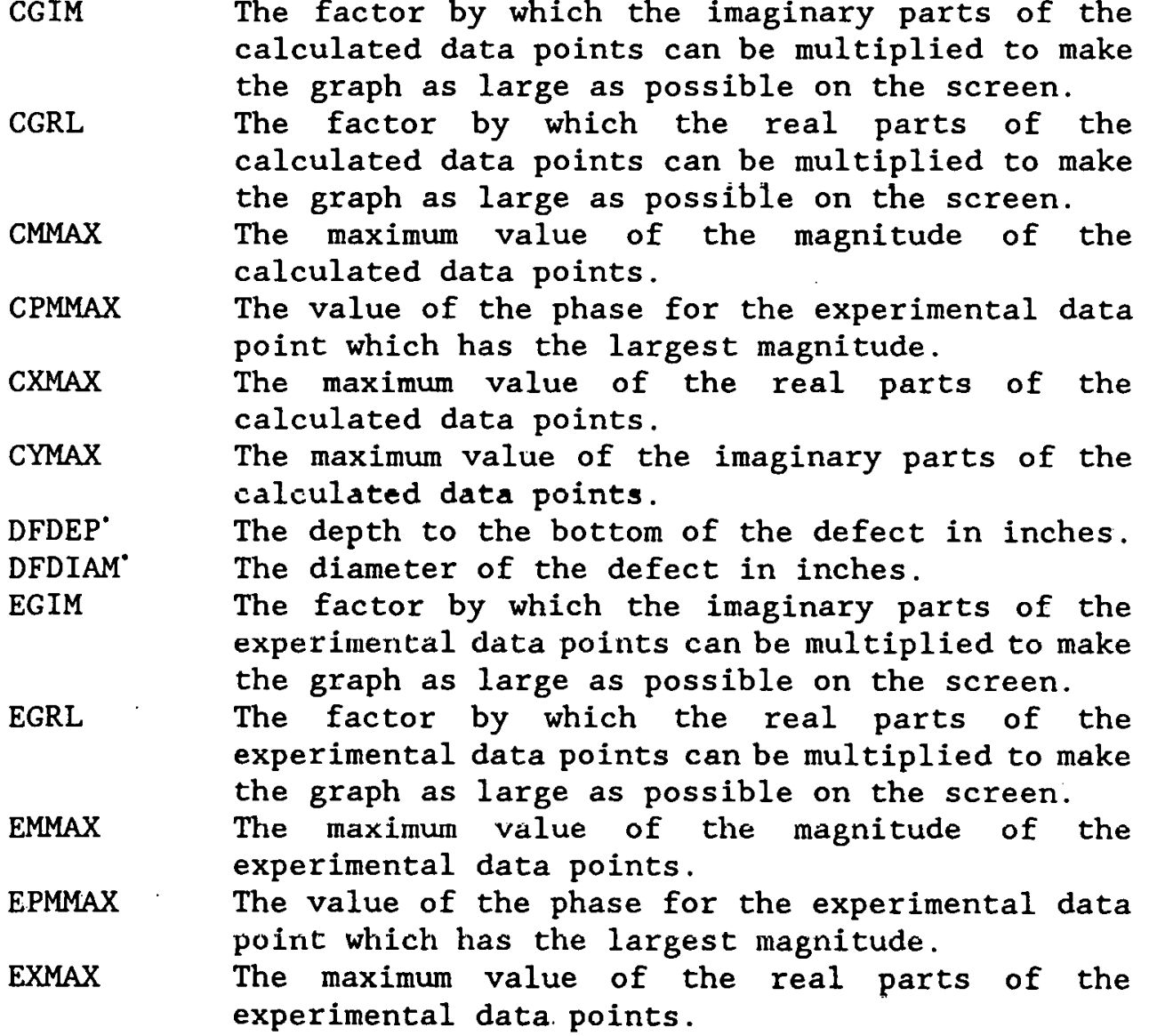


EYMAX The maximum value of the imaginary parts of the experimental data points.

FREQ $\quad$ The operating frequency in hertz.

GAIN $\quad$ Gain of pickup amplifier.

GIM

GRL The factor by which the real parts of both sets

The factor by which the imaginary parts of both sets of data are multiplied to obtain the largest possible graph. of data are multiplied to obtain the largest possible graph.

L3

The normalized length of the driver coil.

L4

L5

L6

$\begin{array}{ll}\text { LOEC }^{*} & \text { The number of the I/O unit connec } \\ & \text { containing the calculated data. } \\ \text { LOEE } & \text { The number of the I/O unit connec }\end{array}$

The normalized length of the pickup coil.

The normalized distance of recess of the pickup coil.

LOEE

The number of the $I / O$ unit connected to the file containing the experimental data.

LOU The number of the $I / O$ unit connected to the printer.

MODE The screen mode to be used. Mode 16 is the EGA high resolution mode.

NPROBE $\quad$ Character variable which contains the name of the reflection probe which is to be used in the calculations.

NS ${ }^{*} \quad$ The side of the plate where the defect is located. If NS $=1$, the defect is on the near side; if NS $=2$, the defect is on the far side.

OIM

The number which is added to the imaginary parts of all data points to move the origin to the desired location.

ORL

The number which is added to the real parts of all data points to move the origin to the desired location.

$\mathrm{RO}^{\circ}$

The output series resistance of the driving amplifier in ohms.

R1

R2

The normalized inner radius of the driver coil.

The normalized outer radius of the driver coil.

The normalized inner radius of the pickup coil.

R4

R5

R6

R7

R9* $^{\circ}$

The normalized outer radius of the pickup coil.

The mean radius of the driver coil in inches.

DC resistance of the driver coil in ohms.

DC resistance of the pickup coil in ohms.

The input shunt resistance of pickup amplifier in ohms.

RHO1 The resistivity of the plate in $\mu \Omega-\mathrm{cm}$.

$\mathrm{T} 1^{\circ}$

The thickness of the plate. It is input in inches and normalized by the program.

TNDR The number of turns in the driver coil.

TNPU The number of turns in the pickup coil. 
$\mathrm{UI}{ }^{\circ}$

VIN"

WUSRR
The relative magnetic permeability of the plate. Output voltage of driving amplifier in volts. The product of the angular frequency, the magnetic permeability, the electrical conductivity, and the square of the mean coil radius. 


\section{Listing}

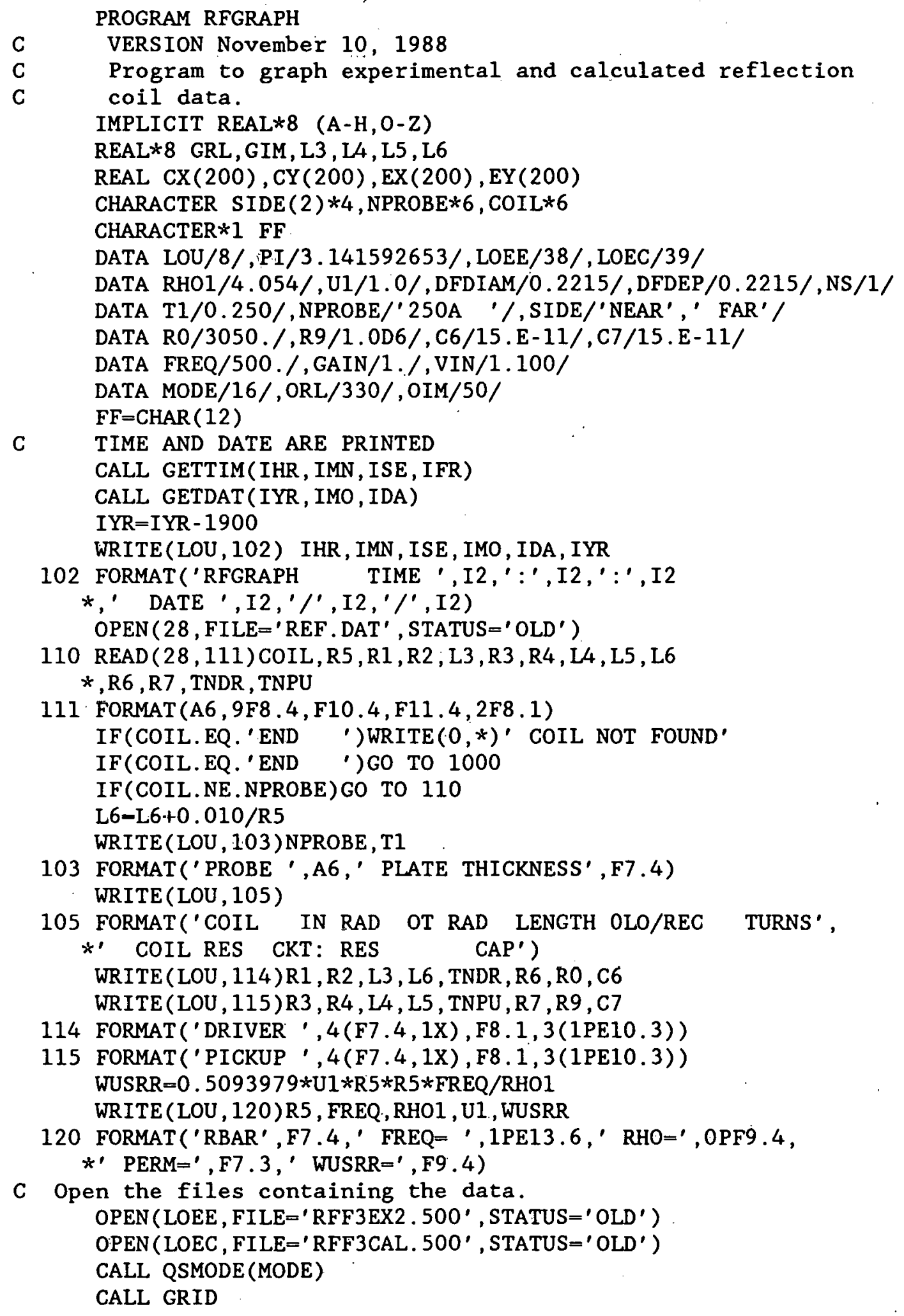


11 CXMAX $=0$.

CYMAX $=0$.

EXMAX $=0$.

EYMAX $=0$.

EMMAX $=0$.

CMMAX $=0$.

$I=1$

$20 \operatorname{READ}($ LOEE , *, END=29) EMAG, EPHA

$E P H A=E P H A * P I / 180$.

$\operatorname{EX}(I)=E M A G * \operatorname{COS}(E P H A)$

$\operatorname{EY}(I)-E M A G * S I N(E P H A)$

$\operatorname{IF}(\mathrm{ABS}$ (EX (I)) .GT. EXMAX) EXMAX =ABS (EX (I))

IF (ABS (EY (I)) . GT . EYMAX) EYMAX $=A B S(E Y(I))$

IF (EMAG.GT . EMMAX) THEN

EMMAX =EMAG

EPMMAX $=$ EPHA

END IF

$\mathrm{I}=\mathrm{I}+1$

GO TO 20

$29 \operatorname{EX}(I)=999$.

$I=1$

30 READ (LOEC , *, END=40) XTMP, CMAG , CPHA

$\mathrm{CPHA}=\mathrm{CPHA} * \mathrm{PI} / 180$.

$\mathrm{CX}(\mathrm{I})=\mathrm{CMAG} * \operatorname{COS}(\mathrm{CPHA})$

$C Y(I)=C M A G * S I N(C P H A)$

$\operatorname{IF}(\operatorname{ABS}(\mathrm{CX}(\mathrm{I}))$. GT . CXMAX) $\mathrm{CXMAX}=\mathrm{ABS}(\mathrm{CX}(\mathrm{I}))$

IF (ABS (CY (I)) . GT . CYMAX) CYMAX =ABS (CY (I))

IF (CMAG , GT , CMMAX) THEN

CMMAX $=$ CMAG

$C P M M A X=C P H A$

END IF

$\mathrm{I}=\mathrm{I}+1$

GO TO 30

$40 \mathrm{CX}(\mathrm{I})=999$.

$\operatorname{EGIM}=300 . / \operatorname{EXMAX}$

EGRL $=300$. / EYMAX

IF(EGIM.GI.EGRL) I'HEN

EGIM=EGRL

ELSE

$E G R L=E G I M$

END IF

CGIM $=300 . /$ CXMAX

CGRL $=300$. / CYMAX

IF(CGIM.GT . CGRL) THEN

CGIM=CGRL

ELSE

CGRL=CGIM.

END IF

IF(EGIM.GT.CGIM) THEN

GIM=CGIM

$G R L=C G R L$ 


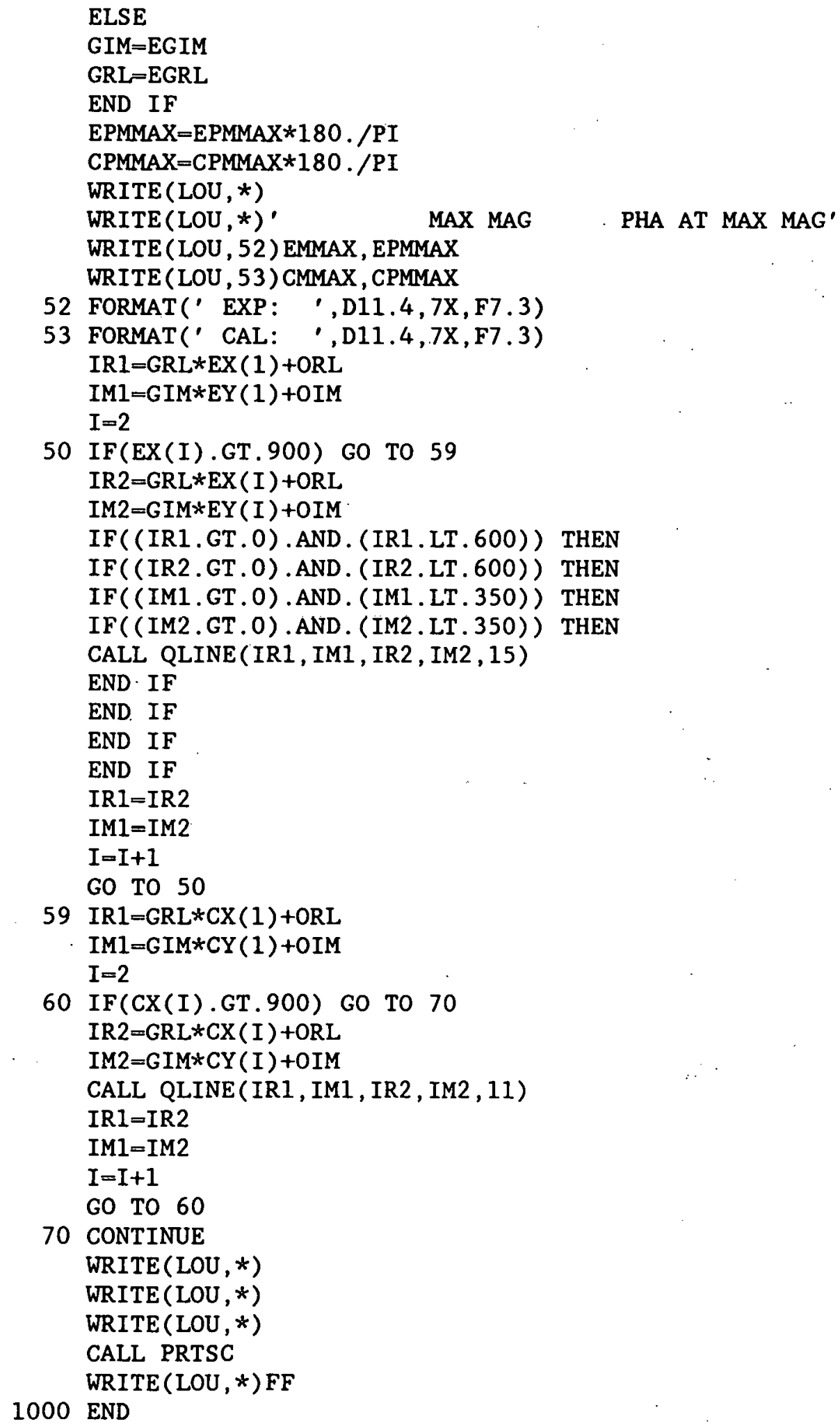


RFINV inverts scan of defect using reflection coil data

Program RFINV calculiates the depth and volume of a defect given the change in induced voltage in a pickup coil caused by the defect. The program works with either experimental data stored in a file by program RFFIX or with calculated data stored by program RFAVZSCN, and it uses a lookup file built by program RFBEDF. The program calculates the integral of the change in induced voltage in the pickup coil due to the presence of a defect with respect to the radial distance between the coil axis and the center of the defect from the inner radius of the pickup coil to the outer radius of the driver coil and then finds the magnitude and phase of this complex integral. It opens a lookup file which contains a list of phases along with the magnitude and depth corresponding to these phases. When the program finds in the lookup file the phase it calculated when doing the integral, the depth corresponding to this phase is the depth of the defect, and the magnitude in the lookup file can be used with the magnitude obtained by doing the integral to find the volume of the defect.

\section{Summary}

1. Declare variable types.

2. Initialize variables.

3. Open the file containing the experimental data, read in the data, and calculate the integral.

4. Find the depth and magnitude in the lookup file corresponding to the phase of the integral.

5. Calculate the inverted defect depth and volume based on the experimental data.

6. Open the file containing the calculated data, read in the data, and calculate the integral.

7. Find the depth and magnitude in the lookup file corresponding to the phase of the integral.

8. Calculate the inverted defect depth and volume based on the calculated data.

$\underline{\text { Variables }}$

Starred variables must be set by the user.

C6 $6^{\circ} \quad$ The total shunt capacitance in farads of the driving circuit.

67 The total shunt rapasitance in farads of the pickup circuit.

DELRDC $\quad$ The normalized distance in the radial direction between data points from the calculated data file.

DELRDE ${ }^{*} \quad$ The normalized distance in the radial direction between data points from the experimental data file.

DELTR $\quad$ The radial distance in inches between points in the experimental data file. 
DEPTH The inverted normalized depth of the center of the defect.

DFDEP $\quad$ The actual depth to the bottom of the defect in inches.

DFDIAM' The actual diameter of the defect in inches.

DFM

DFP

The magnitude of the change in induced voltage in the pickup coil at a point due to the defect.

FREQ

L3

L4

L5

L6 The phase of the change in induced voltage in the pickup coil at a point due to the defect.

The frequency in hertz at which the circuit is driven.

The normalized length of the driver coil.

The normalized length of the pickup coil.

The normalized distance of recess of the pickup coil.

LHSMAG The magnitude of the integral calculated by the program.

LHSPHA The phase of the integral calculated by the program.

LOEC ${ }^{\circ}$ The channel on which the file containing the calculated data is opened.

LOEE $\quad$ The channel on which the file containing the experimental data is opened.

NPROBE $\quad$ Character variable containing the name of the reflection coil being used.

NS

The side of the plate where the defect is located. If $N S=1$, the defect is on the near side; if $N S=2$, the defect is on the far side.

$\mathrm{RO} 0^{\circ}$

$\mathrm{R} 1$

R2

R3

R4

R5

R6

R7

R9.

RDC

The series resistance of the driver circuit.

The normalized inner radius of the driver coil.

The normalized outer radius of the driver coil.

The normalized inner radius of the pickup coil.

The normalized outer radius of the pickup coil.

The mean radius of the driver coil in inches.

DC resistance of the driver coil in ohms.

DC resistance of the pickup coil in ohms.

The amplifier input impedance.

The normalized radial distance between the coil and defect for the calculated inversion section of the program.

RDE The normalized radial distance between the coil and defect for the experimental inversion section of the program.

$\mathrm{RHOI}^{\circ}$ The resistivity of the plate in $\mu \Omega-\mathrm{cm}$.

RHSMAG The magnitude in the lookup file corresponding to the phase of the integral calculated by the program.

T1 The thickness of the plate. It is input in inches and then normalized by the program.

TNDR The number of turns in the driver coil. 
TNPU The number of turns in each pickup coil.

$\mathrm{U} 1^{\circ}$

VOL1

The relative magnetic permeability of the plate.

VOLN

The inverted normalized volume of the defect.

ZD

The actual normalized volume of the defect.

The actual normalized depth of the center of the defect.

Notes

1. If the user wants the program to perform only the inversion of calculated data, he should remove the ' $c$ ' which comments out the statement ' goto 79' just before statement number 70 in the program. If the user wants the program to perform only the inversion of experimental data, he should remove the ' $c$ ' which comments out the statement 'goto $89^{\prime}$ just before statement number 80 in the program. 


\section{Listing}

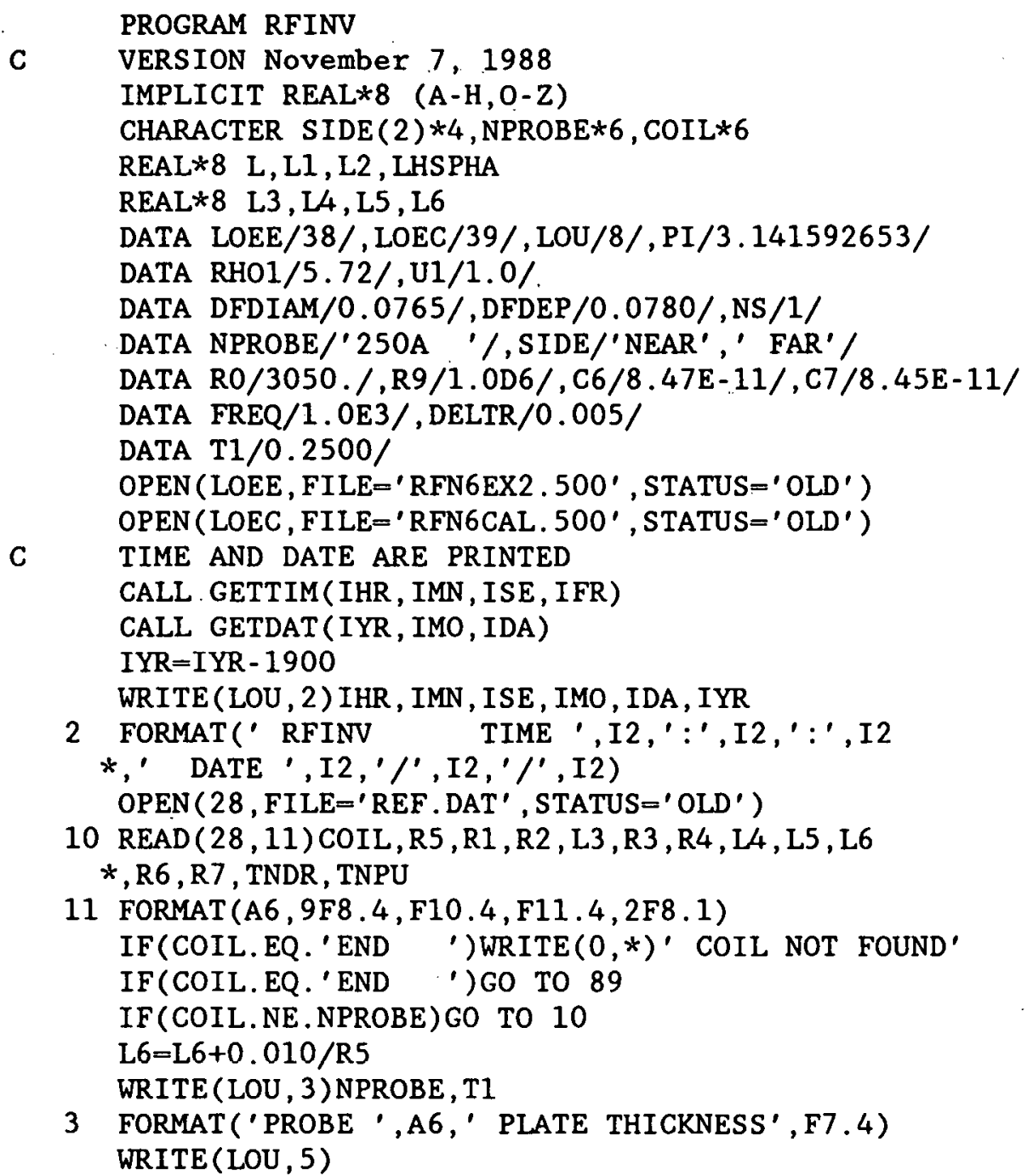

5 FORMAT('COIL IN RAD OT RAD LENGTH OLO/REC TURNS', *' COIL RES CKT: RES CAP') WRITE (LOU , 14)R1, R2 , L3 , L6, TNDR , R6 , R0 , C6 WRITE (LOU , 15)R3, R4, L4, L5, TNPU, R7, R9, C7

14 FORMAT('DRIVER ' 4 (F7.4,1X), F8.1,3(1PE10.3))

15 FORMAT('PICKUP ' , 4(F7.4,1X), F8.1,3(1PE10.3)) WUSRR $=0.5093979 * \mathrm{U} 1 * \mathrm{R} 5 * \mathrm{R} 5 * \mathrm{FREQ} / \mathrm{RHO} 1$ WRITE (LOU, 20)R5, FREQ, RHO1, U1, WUSRR

20 FORMAT ('RBAR', F7.4,' FREQ= ', IPE13.6,' RHO=', OPF9.4, *' PERM=', F7.3,' WUSRR=' ,F9.4)

WRITE (LOD , 23) NPROBE, T1, FREQ, SIDE(NS), DFDIAM, DFDEP WRITE $(0,23)$ NPROBE, T1, FREQ, SIDE (NS), DFDIAM, DFDEP

23 FORMAT('PROBE ' A6.,' PLATE THK' ,F7.4,' FREQ=' , 1PE8.1,1X,A4, *' SIDE' , OPF6.4,' DIA',F6.4,' DEEP')

DELRDC $=0.01 *(R 2-R 3)$

DELRDE $=$ DELTR $/$ R 5 


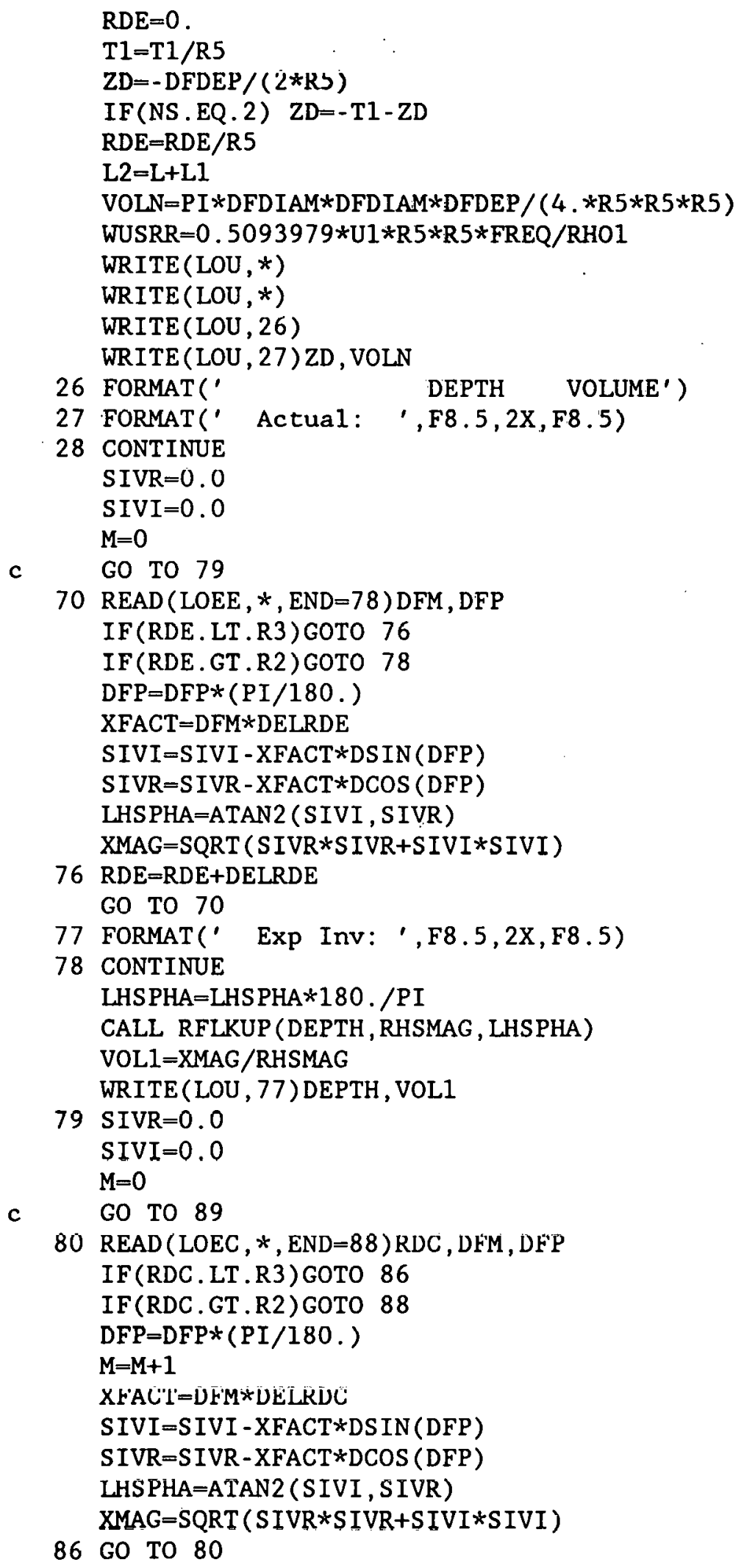


87 FORMAT(' Cal Inv: ', F8.5, 2X, F8.5)

88 CONTINUE

LHSPHA=LHSPHA $* 180 . /$ PI

CALL RFLKUP (DEPTH; RHSMAG, LHSPHA)

VOL1=XMAG/RHSMAG :

WRITE (LOU, 87) DEPTH, VOLI

89 END 
The programs in this section perform functions relating to the effect of a defect in a single conducting tube on a circumferential coil. The types of circumferential coils dealt with are absolute boreside and differential boreside. Fig. 13 shows a differential probe in a tube.

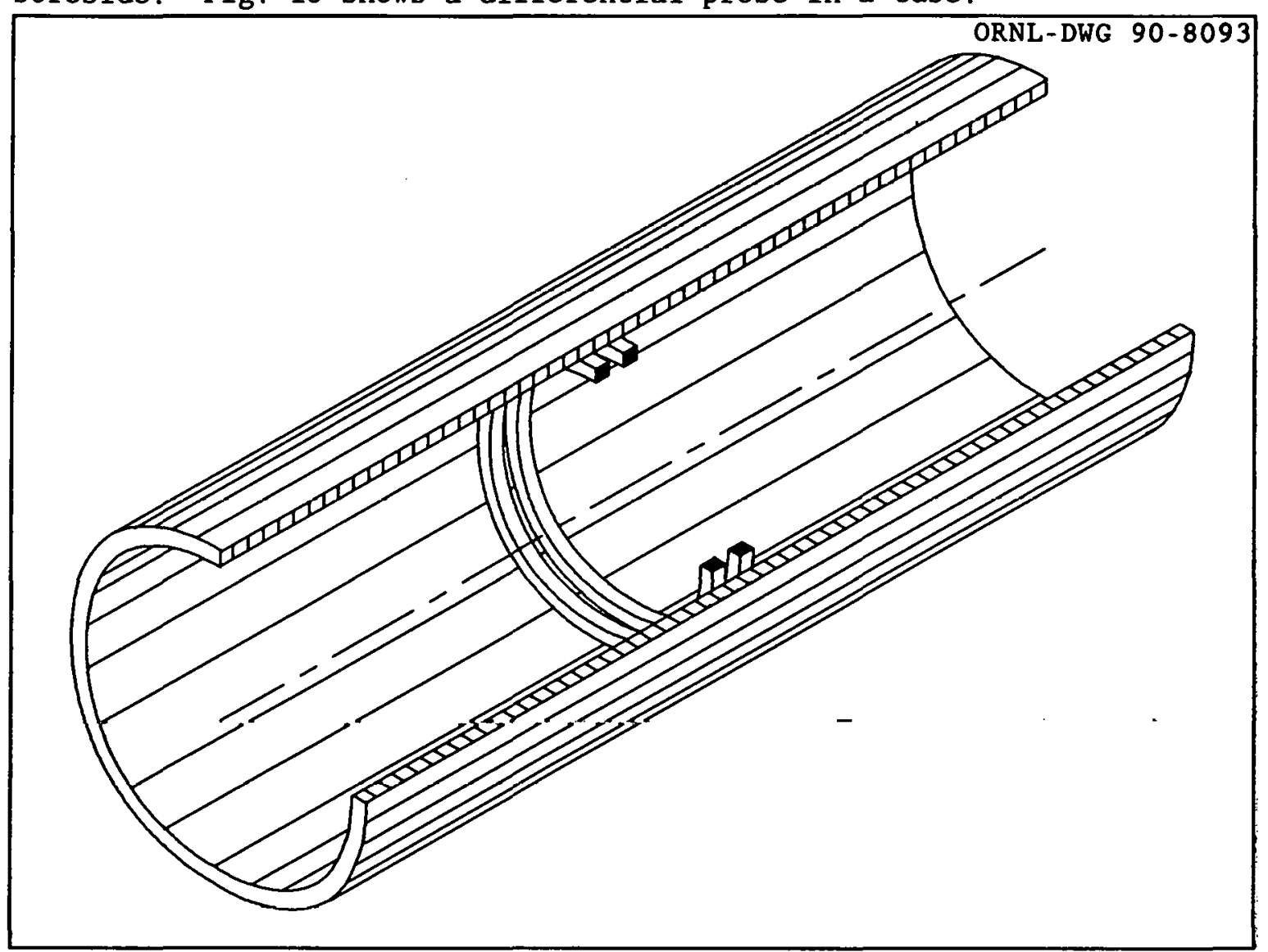

Fig. 13. Differential probe in the bore of a tube.

Calculations of the normalized impedance change in these coils due to a defect in a single conducting tube are done by programs ABBORAR and DFBORAR. The other pair of programs in this section, DBDSF and DBDSFPLT, work together to calculate and plot the contours of the magnitude of the defect sensitivity factor of a differential boreside coil. 
ABBORAR calculates defect impedance change for absolute coil

$\therefore$

The program ABBORAR calculates the change in the normalized impedance of an absolute boreside coil due to the presence of a defect in a single conducting tube, as shown in Fig. 14. It performs the calculations for a number of different axial distances between the center of the coil and the defect, and it can divide the defect into a number of parts and calculate the effect of each part separately.

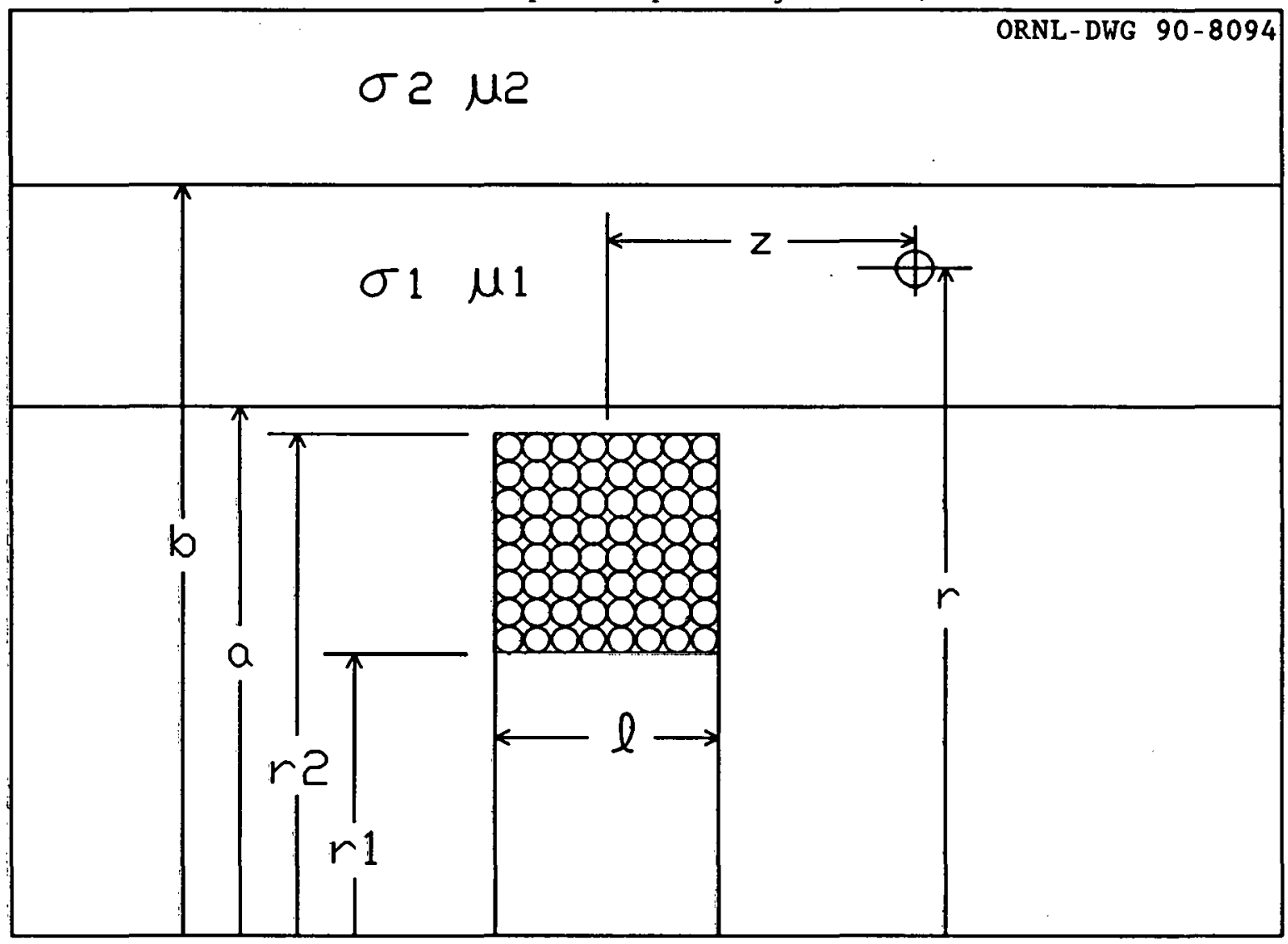

Fig. 14. Cross section of a coil in the bore of a tube with a defect present.

The normalized impedance for a coil inside a cylindrical conductor, with no defect present, is:

$Z_{\mathrm{n}}=\frac{j}{I_{\mathrm{a} \cdot \mathrm{r}}} \int_{i 0}^{\infty}\left[\frac{8}{\pi \alpha^{6}} I^{2}\left(Y_{2}, r_{1}\right)\left\{\frac{K_{1}(\alpha, a) D_{4}-I_{1}\left(\alpha_{1} a\right) D_{1}}{I_{1}(\alpha a)\left(D_{1} D_{2}+D_{3} D_{4}\right)}-\frac{K_{1}(\alpha a)}{I_{n}(\alpha a)}\right\} \sin ^{2}\left(\frac{\alpha \ell}{2}\right)+I_{\mathrm{air}}\right] d \alpha$

and for the change in the normalized impedance due to the defect we have:

$Z_{\mathrm{nd}}(I, z)=\frac{-3\left(\omega \mu \sigma_{1} \overline{\mathrm{r}}^{2}\right) V o I_{\mathrm{n}}}{2 \pi I_{\mathrm{a} \mid \mathrm{r}}}\left[\int_{0}^{\infty} \frac{I\left(r_{2}, r_{1}\right)}{\pi \alpha^{3}}\left\{\frac{K_{1}\left(\alpha_{1} r\right) D_{4}-I_{1}\left(\alpha_{1} r\right) D_{1}}{\left(D_{1} D_{2}+D_{3} D_{4}\right)}\right\} \sin \left(\frac{\alpha \ell}{2}\right) 2 \cos (\alpha z) d \alpha\right]^{2}$ 
where:

$$
\begin{aligned}
& D_{1}=\beta_{2} b K_{0}\left(\alpha_{2} b\right) K_{1}(\alpha, b)-\beta_{1} b K_{0}\left(\alpha_{1} b\right) K_{1}\left(\alpha_{2} b\right) \\
& D_{2}=\beta_{1} a I_{0}(\alpha, a) I_{1}(\alpha a)-\alpha a I_{0}(\alpha a) I_{1}\left(\alpha_{1} a\right) \\
& D_{3}=\alpha a I_{0}(\alpha a) K_{1}\left(\alpha_{1} a\right)+\beta_{1} a K_{0}\left(\alpha_{1} a\right) I_{1}(\alpha a) \\
& D_{4}=\beta_{1} b I_{0}(\alpha, b) K_{1}\left(\alpha_{2} b\right)+\beta_{2} b K_{0}\left(\alpha_{2} b\right) I_{1}(\alpha, b) \\
& I\left(r_{2}, r_{1}\right)=\int_{\alpha r_{1}}^{\alpha r_{2}} x I_{1}(x) d x \\
& \alpha_{1}=\left(\alpha^{2}+j \omega \mu \sigma_{1} \bar{r}^{2}\right)^{1 / 2}
\end{aligned}
$$

and

$$
\beta_{1}=\left(\mu_{0} / \mu_{1}\right)\left(\alpha^{2}+j \omega \mu_{1} \sigma_{i} \bar{r}^{2}\right)^{1 / 2}
$$

where $\quad J\left(r_{2}, r_{1}\right)=\int_{\alpha r_{1}}^{\alpha r_{2}} x \cdot I_{1}(x) d x$

In the computer program, the outer conductor is taken to be air and given a conductivity of zero and a relative permeability of unity. The term $\beta_{2}$ therefore reduces to $\alpha$. The term is carried as $\beta_{2}$ in the derivation and equations for completeness.

\section{$\underline{\text { Varlables }}$}

A number of the variables which occur mainly inside the integration loops do not correspond to anything physical. These variables are described in a section at the end. Starred variables must be assigned by the user.
A
B The normalized outer radius of the tube.
DELTAZ The normalized axial distance between the points at which the calculations are done.
DFDEP $\quad$ The depth of the defect in the tube in inches.
DFDIAM The diameter of the defect in the tube in inches.
DFM The magnitude of the normalized impedance change in the coil due to the defect.
DFP The phase of the normalized impedance change in 
DSFI

DSFR

FREQ

ISIDE

$L^{\circ}$

LOD

LOE $^{*}$

LOU

NRT

NZT*

POW

Q6

R1 ${ }^{\circ}$

$\mathrm{R}^{*}$

R3

$\mathrm{RCL}^{\circ}$

RD

RDT

RHO1 $^{\circ}$

$\mathrm{T} 1^{\circ}$

TRN'

$\mathrm{U} 1^{\circ}$

VOLN

WUSRR

the coil due to the defect.

The imaginary part of the defect sensitivity factor of the coil.

The real part of the defect sensitivity factor of the coil.

The operating frequency in hertz.

The side of the tube where the defect is located. If ISIDE $=1$, the defect is on the outside of the tube; if ISIDE $=2$, the defect is on the inside of the tube.

The length of the coil. The value is input in inches and normalized by the program.

The number of the I/O unit connected to the output data file.

The number of the $I / O$ unit connected to a file which contains the diameters and depths of the defects for which calculations are to be performed.

The number of the $I / O$ unit connected to the printer.

The total number of parts centered along the axis of the defect into which the defect is divided to perform the calculations.

The total number of different values of the axial distance between the center of the coil and the defect for which calculations are performed.

The depth of the defect expressed as a percentage of wall thickness.

The inductance in henries of the coil in air.

The inner radius of the coil. The value is input in inches and normalized by the program.

The outer radius of the coil. The value is input in inches and normalized by the program.

The mean radius of the coil in inches.

The distance between the outside of the coil and the inside wall of the tube. The value is input in inches and normalized by the program.

The radial distance between the center of the coil and the defect (see note 1 ).

The normalized depth to the bottom of the defect. A negative number.

The electrical resistivity of the tube in $\mu \Omega-\mathrm{cm}$. The thickness of the tube wall. The value is input in inches and normalized by the program.

The number of turns in the coil.

The relative magnetic permeability of the tube.

The normalized volume of the defect.

The product of the angular operating frequency, the magnetic permeability of the tube, the electrical conductivity of the tube, and the square of the mean radius of the coil. 
ZD

ZNDFI

ZNDFR

ZNIM

ZNRL

The axial distance between the center of the coil and the defect.

The imaginary part of the normalized impedance change in the coil due to the defect.

The real part of the normalized impedance change in the coil due to the defect.

The imaginary part of the normalized impedance of the coil when no defects are present.

The real part of the normalized impedance of the coil when no defects are present. 
Integration Section of Program ABBORAR

Symbo1 definitions

The following are definitions of the symbols used to describe the program variables which appear in the integration section of the program. All lengths are normalized.unless otherwise noted.

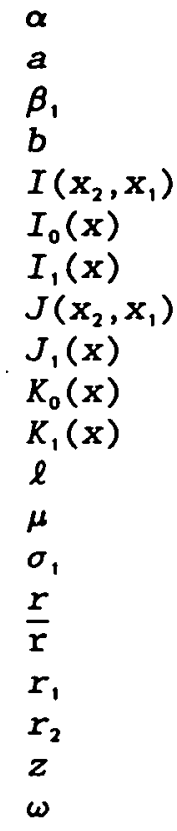

Integration variable

Inner radius of the tube $\left(\alpha^{2}+j \omega \mu \sigma_{1} \overline{\mathrm{r}}^{2}\right)^{1 / 2} \mu$

Outer radius of the tube

Integral of $x I_{1}(x)$ with respect to $x$ from $\alpha x_{1}$ to $\alpha x_{2}$ Modified Bessel function of the first kind of order 0 Modified Bessel function of the first kind of order 1 Integral of $x J_{1}(x)$ with respect to $x$ from $\alpha x_{1}$ to $\alpha x_{2}$ Bessel function of the first kind of order 1 Modified Bessel function of the second kind of order 0 Modified Bessel function of the second kind of order 1 Length of the coil

Relative magnetic permeability of the tube Electrical conductivity of the tube; Radial distance between center of coil and defect Coil mean radius in inches

Inner radius of coil

Outer radius of coil

Axial distance between defect and coil center Angular operating frequency

\begin{tabular}{|c|c|}
\hline $\begin{array}{l}\text { Program } \\
\text { variable }\end{array}$ & $\begin{array}{l}\text { Symbolic } \\
\text { equivalent }\end{array}$ \\
\hline$\overline{\mathrm{A} 1}$ & $\alpha \ell+\exp (-\alpha \ell)-1$ \\
\hline BIOA & $I_{0}(\alpha a)$ \\
\hline BIOB & $I_{0}(\alpha b)$ \\
\hline BIIA & $I_{1}(\alpha a)$ \\
\hline BIIB & $I_{1}(\alpha b)$ \\
\hline BIIIA & $\operatorname{Im}\left[I_{1}(\alpha, a)\right]$ \\
\hline BIIIB & $\operatorname{Im}\left[I_{1}(\alpha, b)\right]$ \\
\hline BIIIR & $\operatorname{Im}\left[I_{1}(\alpha ; r)\right]$ \\
\hline BIIRA & $\operatorname{Re}\left[I_{1}(\alpha, a)\right]$ \\
\hline BIIRB & $\operatorname{Re}[I,(\alpha, b)]$ \\
\hline BIIRR & $\dot{\operatorname{Re}}\left[I_{1}(\alpha, r)\right]$ \\
\hline $\mathrm{BKOA}$ & $K_{0}(\alpha a)$ \\
\hline
\end{tabular}




\begin{tabular}{|c|c|}
\hline BKOB & $K_{0}(\alpha b)$ \\
\hline BK1A & $K_{1}(\alpha a)$ \\
\hline BK1B & $K_{1}(\alpha b)$ \\
\hline BKIIA & $\operatorname{Im}[K,(\alpha, a)]$ \\
\hline BK1IB & $\operatorname{Im}\left[K_{1}(\alpha, b)\right]$ \\
\hline BKIIR & $\operatorname{Im}\left[K_{1}(\alpha, r)\right]$ \\
\hline BK1RA & $\operatorname{Re}\left[K_{1}(\alpha, a)\right]$ \\
\hline BK1RB & $\operatorname{Re}\left[K_{1}(\alpha, b)\right]$ \\
\hline BK1RR & $\operatorname{Re}\left[K_{1}(\alpha, r)\right]$ \\
\hline \multirow[t]{4}{*}{ DDI } & $\operatorname{Im}\left\{\left[\beta_{2} b K_{0}\left(\alpha_{2} b\right) K_{1}\left(\alpha_{1} b\right)-\beta_{1} b K_{0}(\alpha, b) K_{1}\left(\alpha_{2} b\right)\right]\right.$ \\
\hline & {$\left[\beta, a I_{0}(\alpha, a) I_{1}(\alpha a)-\alpha a I_{0}(\alpha a) I_{1}(\alpha, a)\right]+$} \\
\hline & {$\left[\alpha a I_{0}(\alpha a) K_{1}(\alpha, a)+\beta, a K_{0}(\alpha, a) I_{1}(\alpha a)\right]$} \\
\hline & $\left.\left[\beta_{1} b I_{0}(\alpha, b) K_{1}\left(\alpha_{2} b\right)+\beta_{2} b K_{0}\left(\alpha_{2} b\right) I_{1}\left(\alpha_{1} b\right)\right]\right]$ \\
\hline \multirow[t]{4}{*}{ DDR } & $\operatorname{Re}\left\{\left[\beta_{2} b K_{0}\left(\alpha_{2} b\right) K_{1}\left(\alpha_{1} b\right)-\beta_{1} b K_{0}\left(\alpha_{1} b\right) K_{1}\left(\alpha_{2} b\right)\right]\right.$ \\
\hline & {$\left[\beta_{1} a I_{0}(\alpha, a) I_{1}(\alpha a)-\alpha a I_{0}(\alpha a) I_{1}(\alpha, a)\right]+$} \\
\hline & {$\left[\alpha a I_{0}(\alpha a) K_{1}(\alpha, a)+\beta_{1} a K_{0}(\alpha, a) I_{1}(\alpha a)\right]$} \\
\hline & $\left.\left[\beta, b I_{0}\left(\alpha_{1} b\right) K_{1}\left(\alpha_{2} b\right)+\beta_{2} b K_{0}\left(\alpha_{2} b\right) I_{1}(\alpha, b)\right]\right\}$ \\
\hline
\end{tabular}

DFR

$\operatorname{Re}\left\{K_{1}(\alpha, r)\left[\beta, b I_{0}(\alpha, b) K_{1}\left(\alpha_{2} b\right)+\beta_{2} b K_{0}\left(\alpha_{2} b\right) I_{1}(\alpha, b)\right]-\right.$

$$
\begin{aligned}
& \left.I_{1}(\alpha, r)\left[\beta_{2} b K_{0}\left(\alpha_{2} b\right) K_{1}(\alpha, b)-\beta_{1} b K_{0}(\alpha, b) K_{1}\left(\alpha_{2} b\right)\right]\right\} \\
\div & \left\{\left[\beta_{2} b K_{0}\left(\alpha_{2} b\right) K_{1}(\alpha, b)-\beta, b K_{0}(\alpha, b) K_{1}\left(\alpha_{2} b\right)\right]\right. \\
& {\left[\beta_{1} a I_{0}(\alpha, a) I_{1}(\alpha a)-\alpha a I_{0}(\alpha a) I_{1}(\alpha, a)\right]+} \\
& {\left[\alpha a I_{0}(\alpha a) K_{1}(\alpha, a)+\beta_{1} a K_{0}(\alpha, a) I_{1}(\alpha a)\right] } \\
& {\left.\left[\rho_{1} b I_{0}\left(\alpha_{1} b\right) K_{1}\left(\alpha_{2} b\right)+\beta_{2} b K_{0}\left(\alpha_{2} b\right) I_{1}(\alpha, b)\right]\right\} }
\end{aligned}
$$

DI1

$\operatorname{Im}\left[\beta_{2} b K_{0}\left(\alpha_{2} b\right) K_{1}\left(\alpha_{1} b\right)-\beta, b K_{0}\left(\alpha_{1} b\right) K_{1}\left(\alpha_{2} b\right)\right]$

DI2

$\operatorname{Im}\left[\beta_{1} a I_{0}(\alpha, a) I_{1}(\alpha a)-\alpha a I_{0}(\alpha a) I_{1}(\alpha, a)\right]$

DI 3

$\operatorname{Im}\left[\alpha a I_{0}(\alpha a) K_{1}(\alpha, a)+\beta, a K_{0}(\alpha, a) I_{1}(\alpha a)\right]$

DI4

$\operatorname{Im}\left[\beta, b I_{0}(\alpha, b) K_{1}\left(\alpha_{2} b\right)+\beta_{2} b K_{0}\left(\alpha_{2} b\right) I_{1}(\alpha, b)\right]$

DNI

$\operatorname{Im}\left\{K_{1}\left(\alpha_{1} r\right)\left[\beta_{1} b I_{0}(\alpha, b) K_{1}\left(\alpha_{2} b\right)+\beta_{2} b K_{0}\left(\alpha_{2} b\right) I_{1}\left(\alpha_{1} b\right)\right]-\right.$

$$
\left.I_{1}\left(\alpha_{1} r\right)\left\{\beta_{2} b K_{0}\left(\alpha_{2} b\right) K_{1}\left(\alpha_{1} b\right)-\beta, b K_{0}\left(\alpha_{1} b\right) K_{1}\left(\alpha_{2} b\right)\right]\right\}
$$

DNR

$\operatorname{Re}\left(K_{1}\left(\alpha_{1} r\right)\left[\beta, b I_{0}(\alpha, b) K_{1}\left(\alpha_{2} b\right)+\beta_{2} b K_{0}\left(\alpha_{2} b\right) I_{1}(\alpha, b)\right]-\right.$

$$
\left.I_{1}(\alpha, r)\left[\beta_{2} b K_{0}\left(\alpha_{2} b\right) K_{1}\left(\alpha_{1} b\right)-\beta, b K_{0}\left(\alpha_{1} b\right) K_{1}\left(\alpha_{2} b\right)\right]\right\}
$$




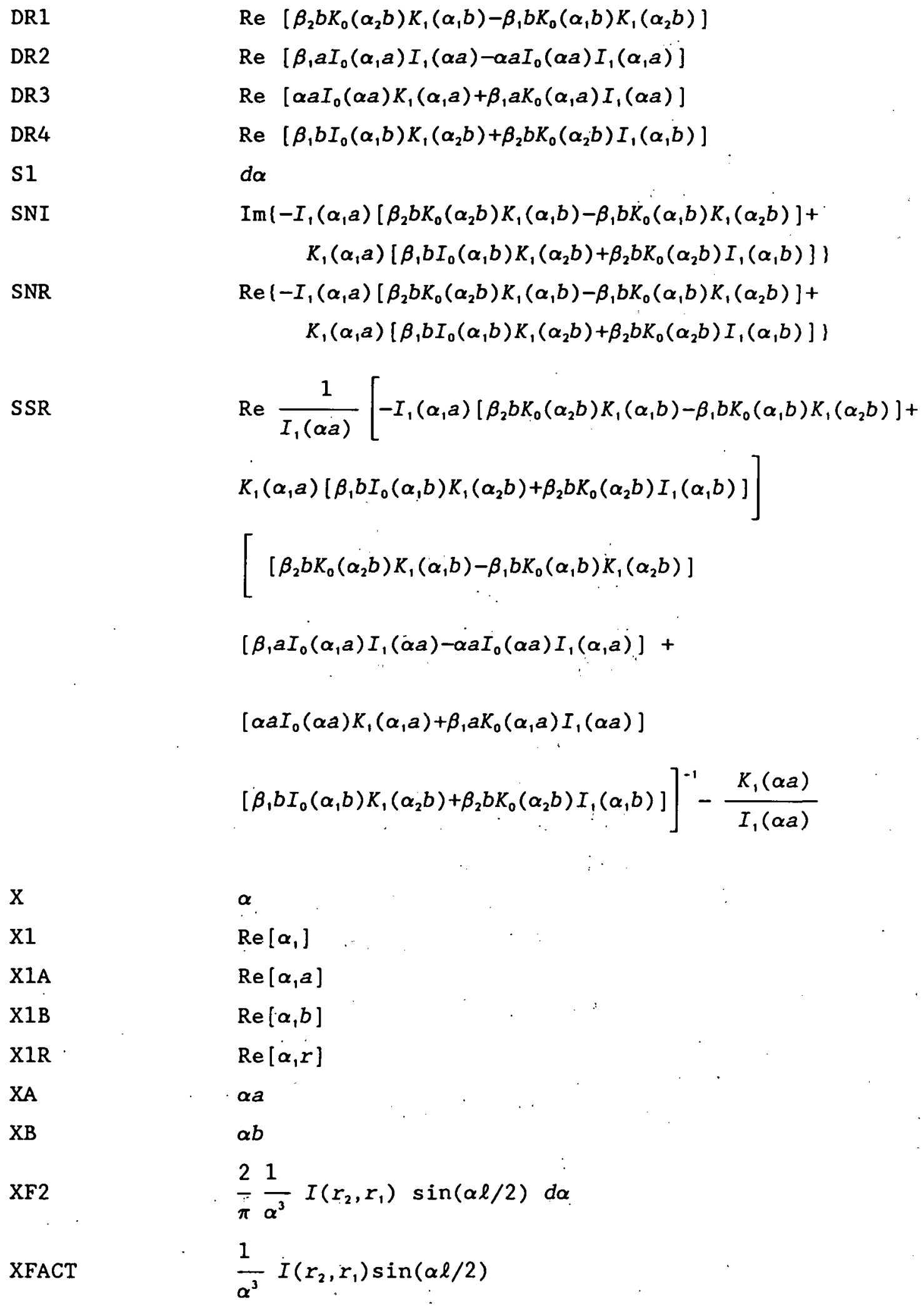


XFACT2

$\frac{2}{\pi} \frac{1}{\alpha^{3}} I\left(r_{2}, r_{1}\right) \sin (\alpha \ell / 2) \cos (\alpha z) d \alpha$

XIR21

$\frac{1}{\alpha^{3}} I\left(r_{2}, r_{1}\right)$

XJR21

$\frac{1}{\alpha^{3}} J\left(r_{2}, r_{1}\right)$

XL

$\alpha \ell$

$\mathrm{XX}$

$\alpha^{2}$

$\mathrm{XxXX}$

$\alpha^{4}$

Y1

$\operatorname{Im}\left[\alpha_{1}\right]$

Y1A

$\operatorname{Im}[\alpha, a]$

Y1B

$\operatorname{Im}[\alpha, b]$

Y1R

$\operatorname{Im}[\alpha, r]$

ZIOIA

$\operatorname{Im}\left[\alpha, a I_{0}(\alpha, a)\right]$

ZIOIB

$\operatorname{Im}\left[\alpha_{1} b I_{0}\left(\alpha_{1} b\right)\right]$

ZIOIR

$\operatorname{Im}\left[\alpha, r I_{0}(\alpha, r)\right]$

ZIORA

$\operatorname{Re}\left[\alpha, a I_{0}(\alpha, a)\right]$

ZIORB

$\operatorname{Re}\left[\alpha, b I_{0}(\alpha, b)\right]$

ZIORR

$\operatorname{Re}\left[\alpha, r I_{0}(\alpha, r)\right]$

ZKOIA

$\operatorname{Im}\left[\alpha, a K_{0}(\alpha, a)\right]$

ZKOIB

$\operatorname{Im}\left[\alpha_{1} b K_{0}(\alpha, b)\right]$

ZKOIR

$\operatorname{Im}\left[\alpha, r K_{0}(\alpha, r)\right]$

ZKORA

$\operatorname{Re}\left[\alpha_{1} a K_{0}\left(\alpha_{1} a\right)\right]$

ZKORB

$\operatorname{Re}\left[\alpha, b K_{0}(\alpha, b)\right]$

ZKORR

$\operatorname{Re}\left[\alpha_{1} r K_{0}(\alpha, r)\right]$

Sample output

The program ABBORAR calculates the defect signal averaged over the depth of the defect at different distances along the tube. The program can plot the defect imperance as the tube is scanned and pick out the maximum amplitude. Below we show a sample run where the maximum signal is print.e.

ABBORAR TIME 8:53:41 DATE 8/16/89

IN RAD OT RAD LENGTH RAD CLR WALLTH \& WALL TUB IR TUB OR

$\begin{array}{lllllllll}\text { ACT } & 1.2000 & 1.5000 & 0.2650 & 0.0575 & 0.2200 & 45.45 & 1.5575 & 1.7775\end{array}$

$\begin{array}{lllllllll}\text { NOR } & 0.8889 & 1.1111 & 0.1963 & 0.0426 & 0.1630 & 45.45 & 1.1537 & 1.3167\end{array}$

RBAR $1.3500 \mathrm{FREQ}=4.000000 \mathrm{E}+02 \mathrm{RHO}=3.8400 \mathrm{PERM}=1.000$ WUSRR $=96.7060$ 
NORM IMPD:RL 0.110847 IM 0.589927 AIR IND 1.693801E-02

NORM DSF:RL 1.4332E-01 IM 3.3396E-02, VOLN 3.1922E-04

MAXIMUM MAG 0.5544D-04

PHA AT MAX MAG 18.91 OD DEFECT

Partial listing of file FORT39:

$\begin{array}{lll}0.000 & 0.5544 \mathrm{D}-04 & 18.91 \\ 0.001 & 0.5544 \mathrm{D}-04 & 18.91 \\ 0.002 & 0.5544 \mathrm{D}-04 & 18.91 \\ 0.003 & 0.5543 \mathrm{D}-04 & 18.91 \\ 0.004 & 0.5543 \mathrm{D}-04 & 18.90 \\ 0.005 & 0.5542 \mathrm{D}-04 & 18.90 \\ 0.006 & 0.5541 \mathrm{D}-04 & 18.89 \\ 0.007 & 0.5540 \mathrm{D}-04 & 18.88 \\ 0.008 & 0.5538 \mathrm{D}-04 & 18.87 \\ 0.009 & 0.5536 \mathrm{D}-04 & 18.86 \\ 0.010 & 0.5535 \mathrm{D}-04 & 18.85\end{array}$




\section{Listing}

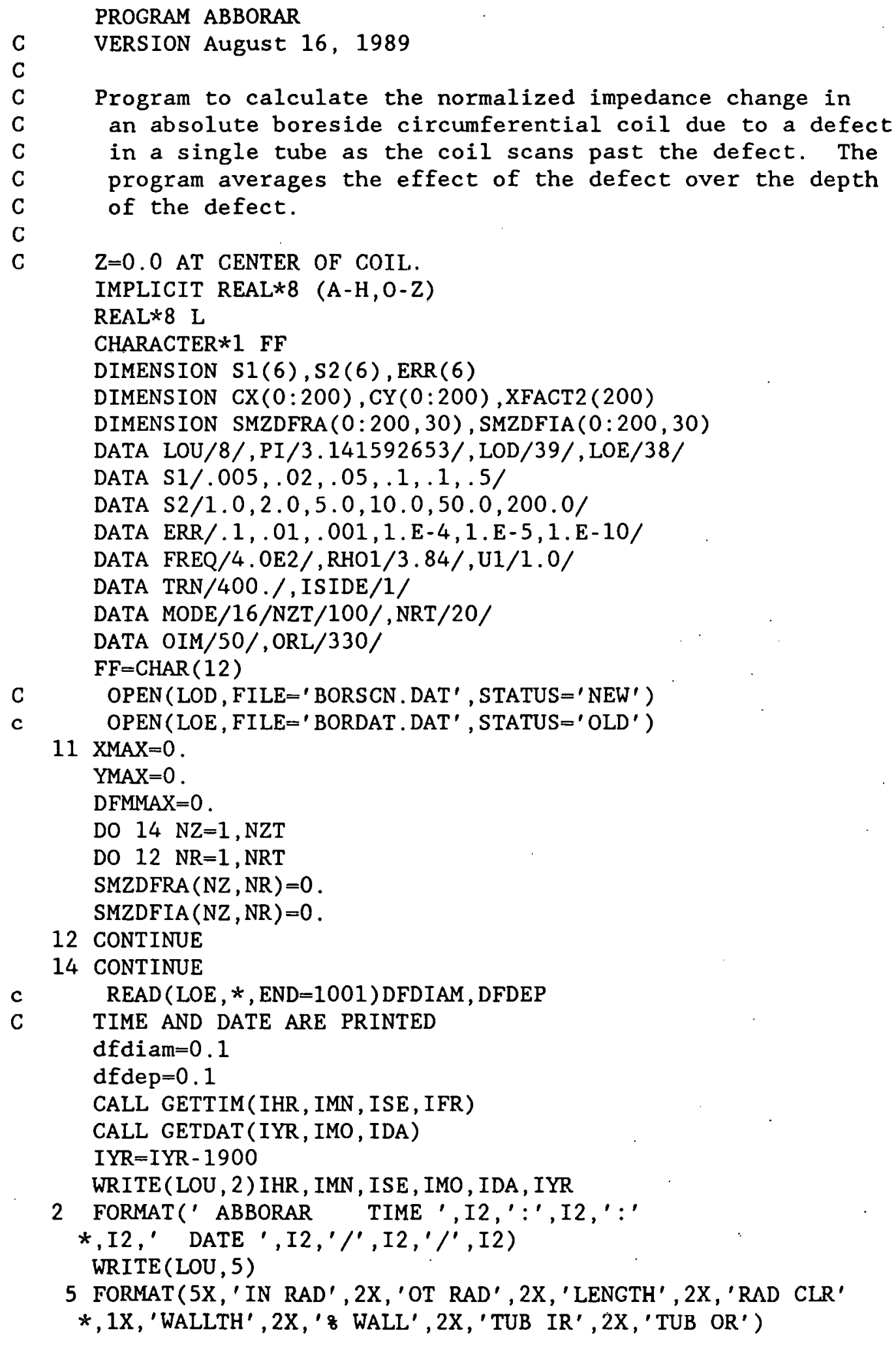




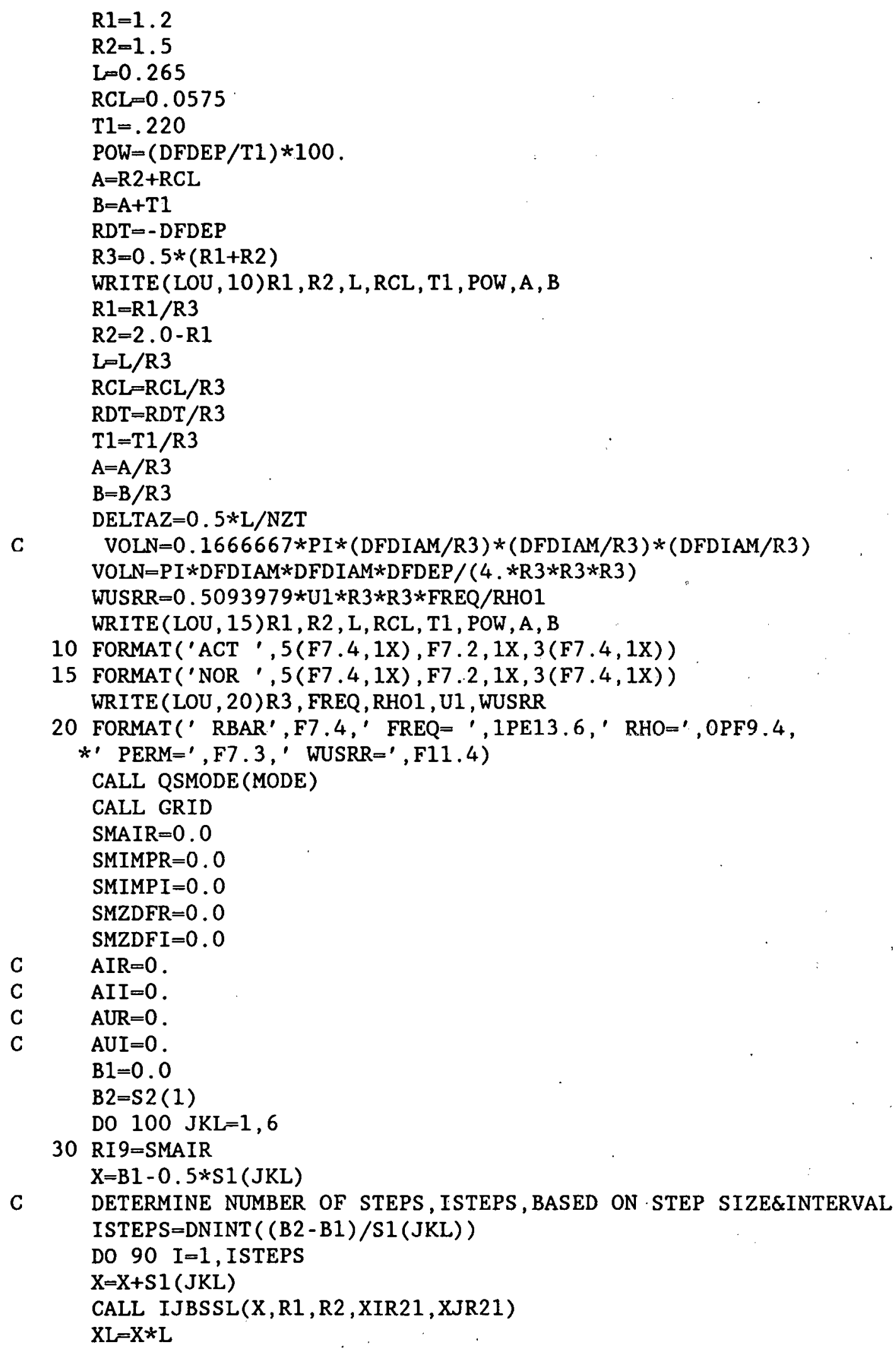


IF (XL.GT . 5.OE-3) GO TO 60

$\mathrm{Al}=\mathrm{XL} * \mathrm{XL} *(0.5-\mathrm{XL} / 6.0)$

GO TO 80

60 IF(XL.GT.75.0) GO TO 70

$\mathrm{AI}=\mathrm{XL}+\mathrm{DEXP}(-\mathrm{XL})-1.0$

GO TO 80

$70 \mathrm{Al}=\mathrm{XL}-1.0$

80 SMAIR=SMAIR $+2 . * X J R 21 * X \mathrm{JR} 21 * A I * \operatorname{Sil}(\mathrm{JKL})$

IF (X.GT. 160.)GO TO 90

$\mathrm{XX}=\mathrm{X} * \mathrm{X}$

$\mathrm{XXXX}=\mathrm{XX} * \mathrm{XX}$

$\mathrm{XI}=\mathrm{DSQRT}(0.5 *(\mathrm{XX}+\mathrm{DSQRT}(\mathrm{XXXX}+\mathrm{WUSRR} * \mathrm{WUSRR}))) / \mathrm{U} 1$

$\mathrm{YI}=\mathrm{WUSRR} /(2 * \mathrm{X} 1 * \mathrm{U} 1 * \mathrm{U} 1)$

$\mathrm{XA}=\mathrm{X} * \mathrm{~A}$

$\mathrm{XB}=\mathrm{X} * \mathrm{~B}$

$\mathrm{XIA}=\mathrm{X} 1 * \mathrm{~A}$

$\mathrm{Y} 1 \mathrm{~A}=\mathrm{Y} 1 * \mathrm{~A}$

$\mathrm{X} 1 \mathrm{~B}=\mathrm{X} 1 * \mathrm{~B}$

$\mathrm{Y} 1 \mathrm{~B}=\mathrm{Y} 1 * \mathrm{~B}$

CALL CMDBES (X1A, Y1A, ZIORA, ZIOIA, ZKORA, ZKOIA, BI1RA, BI1IA

*, BKIRA, BKIIA)

CALL CMDBES (X1B , Y1B , ZIORB , ZIOIB ,ZKORB., ZKOIB , BIIRB , BIIIB

*, BK1RB, BK1IB)

CALL BESI (XA, BIOA, BIIA)

CALL BESK (XA, BKOA, BK1A)

CALL BESI (XB, BIOB, BI1B)

CALL BESK ( $\mathrm{XB}, \mathrm{BKOB}, \mathrm{BK} 1 \mathrm{~B})$

DR.1 $=\mathrm{XB} * \mathrm{BY} . \mathrm{OB} * \mathrm{BK} .1 \mathrm{RB}-\mathrm{ZK} . \mathrm{OB} . \mathrm{B} * \mathrm{BK} .1 \mathrm{~B} / \mathrm{U} 1$

$\mathrm{DI} 1=\mathrm{XB} * \mathrm{BKOB} * \mathrm{BK} 1 \mathrm{IB}-\mathrm{ZKOIB} * \mathrm{BK} 1 \mathrm{~B} / \mathrm{U1}$.

$D R 2=Z I O R A * B I 1 A / U 1-X A * B I O A * B I 1 R A$

DI2 $=Z I O I A * B I 1 A / U 1-X A * B I O A * B I I I A$.

$\mathrm{DR} 3=\mathrm{XA} * \mathrm{BIOA} * \mathrm{BK} 1 \mathrm{RA}+\mathrm{ZKORA} * \mathrm{BIIA} / \mathrm{U1}$

$\mathrm{DI} 3=\mathrm{XA} * \mathrm{BIOA} * \mathrm{BK} 1 \mathrm{IA}+\mathrm{ZKOIA} * \mathrm{BI} 1 \mathrm{~A} / \mathrm{U} 1$

$D R 4=Z I O R B * B K 1 B / U 1+X B * B K O B * B I 1 R B$

$\mathrm{DI} 4=\mathrm{ZIOIB} * \mathrm{BK} 1 \mathrm{~B} / \mathrm{U} 1+\mathrm{XB} * \mathrm{BKOB} * \mathrm{BIIIB}$.

$\mathrm{DDR}=\mathrm{DR} 1 * \mathrm{DR} 2-\mathrm{DI} 1 * \mathrm{DI} 2+\mathrm{DR} 3 * \mathrm{DR} 4-\mathrm{DI} 3 * \mathrm{DI} 4$

$\mathrm{DDI}=\mathrm{DR} 1 * \mathrm{DI} 2+\mathrm{DR} 2 * \mathrm{DI} 1+\mathrm{DR} 3 * \mathrm{DI} 4+\mathrm{DR} 4 * \mathrm{DI} 3$

$S N R=-B I 1 R A * D R 1+B I 1 I A * D I 1+B K 1 R A * D R 4-B K 1 I A * D I 4$

$S N I=-B I 1 I A * D R 1-B I 1 R A * D I 1+B K 1 R A * D I 4+B K 1 I A * D R 4$

$\mathrm{DEN}=\mathrm{DDR} * \mathrm{DDR}+\mathrm{DDI} * \mathrm{DDI}$

$S S R=((S N R * D D R+S N I * D D I) / D E N-B K I A) / B I I A$

$\mathrm{SSI}=(\mathrm{SNI} * \mathrm{DDR}-\mathrm{SNR} * \mathrm{DDI}) /(\mathrm{BI} 1 \mathrm{~A} * \mathrm{DEN})$

$\mathrm{XFACT}=\mathrm{XIR} 21 * \mathrm{DSIN}(\mathrm{XL} / 2$.

SMIMPR $=S M I M P R+8 *$ XFACT $* X F A C T * S 1(\mathrm{JKL}) * S S R / P I$

SMIMPI $=$ SMIMPI $+8 *$ XFACT $* X F A C T * S 1:(\mathrm{JKL}) * S S I / P I$

$\mathrm{XF} 2=2 . * \mathrm{XFACT} * \mathrm{~S} 1(\mathrm{JKL}) / \mathrm{PI}$

DO $89 \mathrm{NR}=1$, NRT

C write $(0, *)$ rdt

$\mathrm{RD}=(\operatorname{REAL}(\mathrm{NR})-0.5) *(\mathrm{RDT} / \mathrm{REAL}(\mathrm{NRT}))$

C write $(0, *)$ rd, rdt 
C

pause

IF (ISIDE . EQ. 1) THEN

$R D=B+R D$

ELSE

$\mathrm{RD}=\mathrm{A}-\mathrm{RD}$

END IF

c

$\mathrm{rd}=1.3333$

$\mathrm{X} 1 \mathrm{R}=\mathrm{X} 1 * \mathrm{RD}$

$\mathrm{Y} 1 \mathrm{R}=\mathrm{Y} 1 * \mathrm{RD}$

CALL CMDBES (X1R, Y1R, ZIORR, ZIOIR, ZKORR, ZKOIR, BIIRR, BI1IR *, BKIRR, BK1IR)

$\mathrm{DNR}=\mathrm{BK} 1 \mathrm{RR} * \mathrm{DR} 4-\mathrm{BK} 1 \mathrm{IR} * \mathrm{DI} 4-\mathrm{BI} 1 \mathrm{RR} * \mathrm{DR} 1+\mathrm{BI} 1 \mathrm{IR} * \mathrm{DI} 1$

$\mathrm{DNI}=\mathrm{BK} 1 \mathrm{IR} * \mathrm{DR} 4+\mathrm{BK} 1 \mathrm{RR} * \mathrm{DI} 4-\mathrm{BI} 1 \mathrm{IR} * \mathrm{DR} 1-\mathrm{BI} 1 \mathrm{RR} * \mathrm{DI} 1$

$\mathrm{DFR}=(\mathrm{DNR} * \mathrm{DDR}+\mathrm{DNI} * \mathrm{DDI}) / \mathrm{DEN}$

$\mathrm{DFI}=(\mathrm{DNI} * \mathrm{DDR}-\mathrm{DNR} * \mathrm{DDI}) / \mathrm{DEN}$

DO $88 \mathrm{NZ}=0, \mathrm{NZT}$

IF (NR.GT. 1)GO TO 87

$\mathrm{ZD}=\mathrm{DELTAZ} * \mathrm{NZ}$

$\mathrm{XFACT} 2(\mathrm{NZ})=\mathrm{XF} 2 * \mathrm{DCOS}(\mathrm{X} * \mathrm{ZD})$

$87 \operatorname{SMZDFRA}(\mathrm{NZ}, \mathrm{NR})=\operatorname{SMZDFRA}(\mathrm{NZ}, \mathrm{NR})+\mathrm{XFACT} 2(\mathrm{NZ}) * \mathrm{DFR}$

$\operatorname{SMZDFIA}(N Z, N R)=\operatorname{SMZDFIA}(\mathrm{NZ}, \mathrm{NR})+\mathrm{XFACT} 2(\mathrm{NZ}) * \mathrm{DFI}$

88 CONTINUE

89 CONTINUE

90 CONTINUE

$\mathrm{B} 1=\mathrm{B} 2$

$\mathrm{B} 2=\mathrm{B} 2+\mathrm{S} 2$ (JKL)

CHECK $=($ SMAIR - RI9 $) /$ SMAIR

IF (ABS (CHECK) . GT.ERR (JKL)) GO TO 30

100 CONTINUE

DO $990 \mathrm{NZ}=0, \mathrm{NZT}$

$\mathrm{ZD}=\mathrm{DELTAZ} * \mathrm{NZ}$

SMZDFR $=0$.

SMZDFI $=0$.

DO $120 \mathrm{NR}=1$, NRT

SMZDFR=SMZDFR+SMZDFRA $(N Z, N R)$

SMZDFI $=$ SMZDFI+SMZDFIA $(\mathrm{NZ}, \mathrm{NR})$

120 CONTINUE

SMZDFR $=$ SMZDFR/NRT

SMZDFI $=$ SMZDFI $/$ NRT

C

WRITE (LOU * *) NZ, SMZDFR, SMZDFI

$\mathrm{DSFR}=-1.5 *$ WUSRR $*($ SMZDFR $*$ SMZDFR - SMZDFI $*$ SMZDFI $) /($ SMAIR $* \mathrm{PI})$

DSFI $=-1.5 *$ WUSRR $* 2.0 *$ SMZDFR $*$ SMZDFI $/($ SMAIR $* P I)$

$135 \mathrm{Q} 6=0.0254 * 4.0 \mathrm{E}-7 * \mathrm{TRN} * \mathrm{TRN} * \mathrm{R} 3 * \mathrm{PI} * \mathrm{PI} * \mathrm{SMAIR} /(\mathrm{L} *(\mathrm{R} 2-\mathrm{R} 1)) * * 2$

ZNIM=SMIMPR/SMAIR +1.0

ZNRL $=-$ SMIMPI/SMAIR

$\mathrm{ZNDFR}=\mathrm{VOLN} * \mathrm{DSFR}$

ZNDFI $=$ VOLN $*$ DSFI

IF(NZ.EQ. 0) WRITE(LOU, 140) ZNRL, ZNIM, Q6 


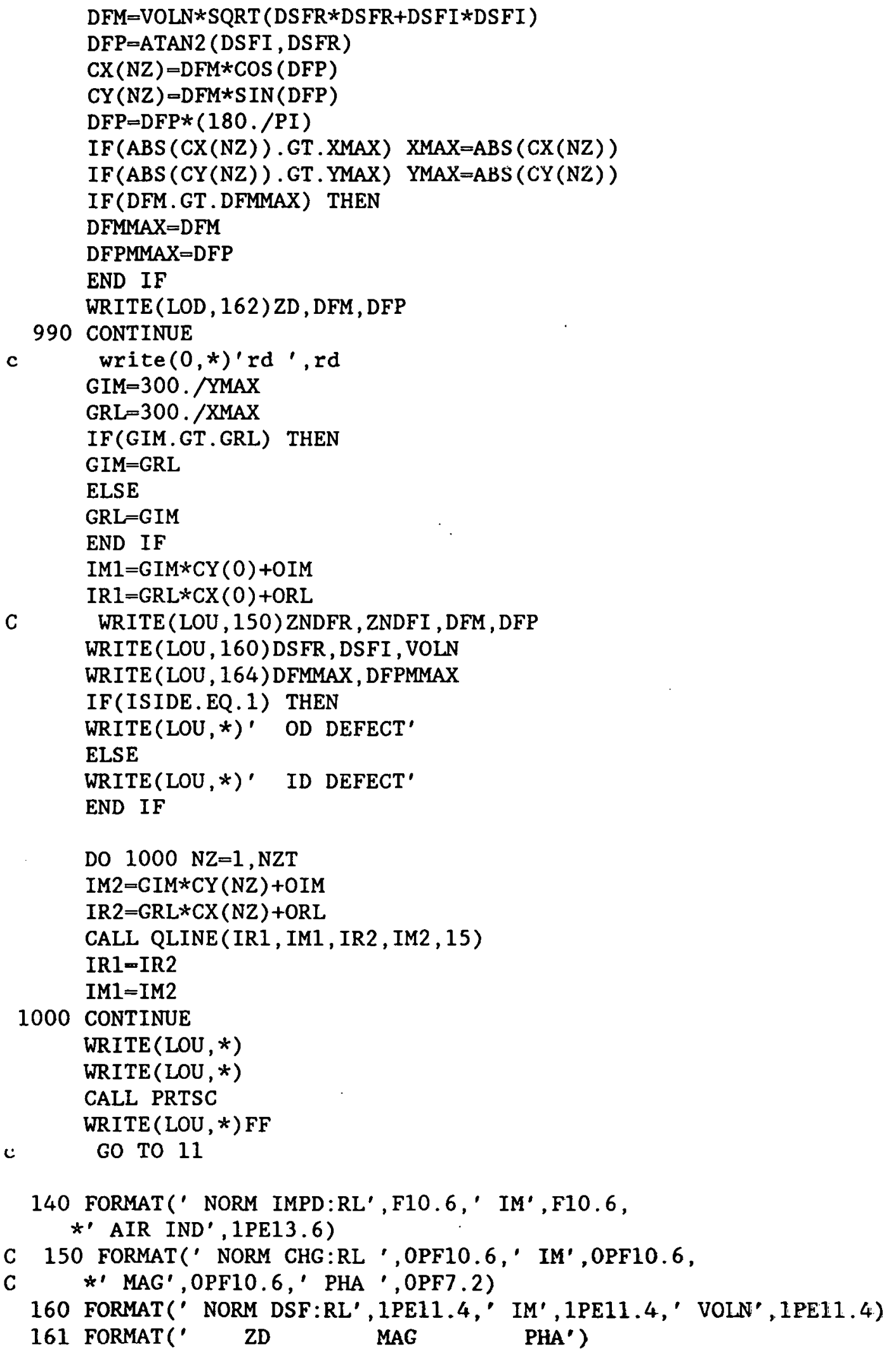


162 FORMAT (F6. 3, 5X, D11.4, 5X, F7.2)

164 FORMAT(' MAXIMUM MAG ',D11.4,' PHA AT MAX MAG ',F7.2, )

1001 END 
DBDSF calculates DSF at lattice of points for differential coil

Program DBDSF calculates the defect sensitivity factor of a differential boreside coil at a two-dimensional lattice of points throughout the wall of a conducting tube. The differential probe is shown in Fig. 15.

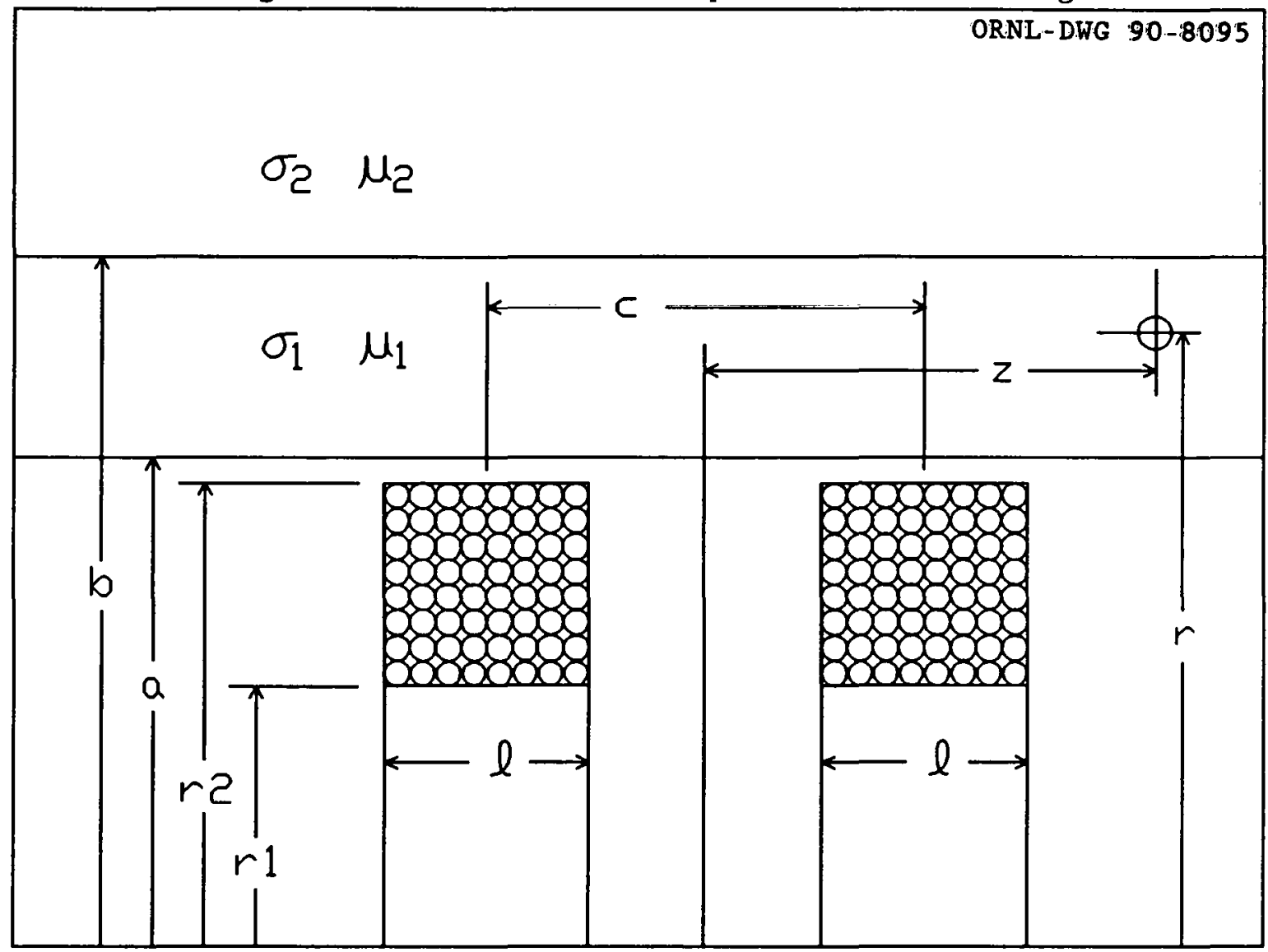

Fig.. 15. Cross section of a differential coil in the bore of a tube with a defect present.

The distance to the defect is measured from the center of the coil assembly, and the center-to-center distance of the coils is denoted $c$. The impedance difference between the two matched coils is:

$$
Z_{\text {nd }}=Z_{1 \mathrm{~d}}-Z_{2 \mathrm{~d}}
$$

Substituting in from Eq. (29) for the impedance change of each coil, removing the normalized defect volume to get the defect sensitivity factor and using some trigonometric identities gives for the defect sensitivity factor: 


$$
\begin{aligned}
D S F(r, z)= & \frac{-3\left(\omega \mu \sigma_{1} \overline{\mathrm{r}}^{2}\right)}{2 \pi I_{\mathrm{alr}}} \\
\times & {\left[\int_{0}^{\infty} \frac{I\left(r_{2}, r_{1}\right)}{\pi \alpha^{3}}\left\{\frac{\left.K_{1}(\alpha, r) D_{4}-I_{1}\left(\alpha_{1} r\right) D_{1}\right)}{\left(D_{1} D_{2}+D_{3} D_{4}\right)}\right\} \sin \left(\frac{\alpha \ell}{2}\right) \sin \left(\frac{\alpha c}{2}\right) \sin (\alpha z) 4 d \alpha\right] } \\
\times & {\left[\int_{0}^{\infty} \frac{I\left(r_{2}, r_{1}\right)}{\pi \alpha^{3}}\left\{\frac{K_{1}\left(\alpha_{1} r\right) D_{4}-I_{1}\left(\alpha_{1} r\right) D_{1}}{\left(D_{1} D_{2}+D_{3} D_{4}\right)}\right\} \sin \left(\frac{\alpha \ell}{2}\right) \cos \left(\frac{\alpha c}{2}\right) \cos (\alpha z) 4 d \alpha\right] }
\end{aligned}
$$

The definitions of the various terms in Eq. (40) are given in Eqs. (30) through (38) in the discussion of the absolute boreside coil. The function and variable names are essentially the same as they are for the absolute coil and will not be repeated here. The program DBDSF stores the calculated values in the data file FORT 40 so they can be plotted by program DBDSFPLT.

\section{Variables}

A
B
C

DELTAR

DELTAZ:

DSFI

DSFM

DSFP

DSFR

FREQ

L:

LOD:

LOU

NRT*

NZT*
The normalized inner radius of the tube.

The normalized outer radius of the tube.

The axial distance between the centers of the two coils. The value is input in inches and normalized by the program.

The normalized radial distance between the points at which the calculations are done.

The normalized axial distance between the points at which the calculations are done.

The imaginary part of the defect sensitivity factor of the coil.

The magnitude of the defect sensitivity factor of the coil.

The phase of the defect sensitivity factor of the coili.

The real part of the defect sensitivity factor of the coill.

The operating frequency in hertz..

The length of each coil. The value is input in inches and normalized by the program.

The number of the $I / 0$ unit connected to the output data file.

The number of the $I / 0$ unit connected to the printer.

The total number of points in the radial direction at which the defect sensitivity factor is calculated.

The total number of points: in the axial direction at which the defect sensitivity factor is 


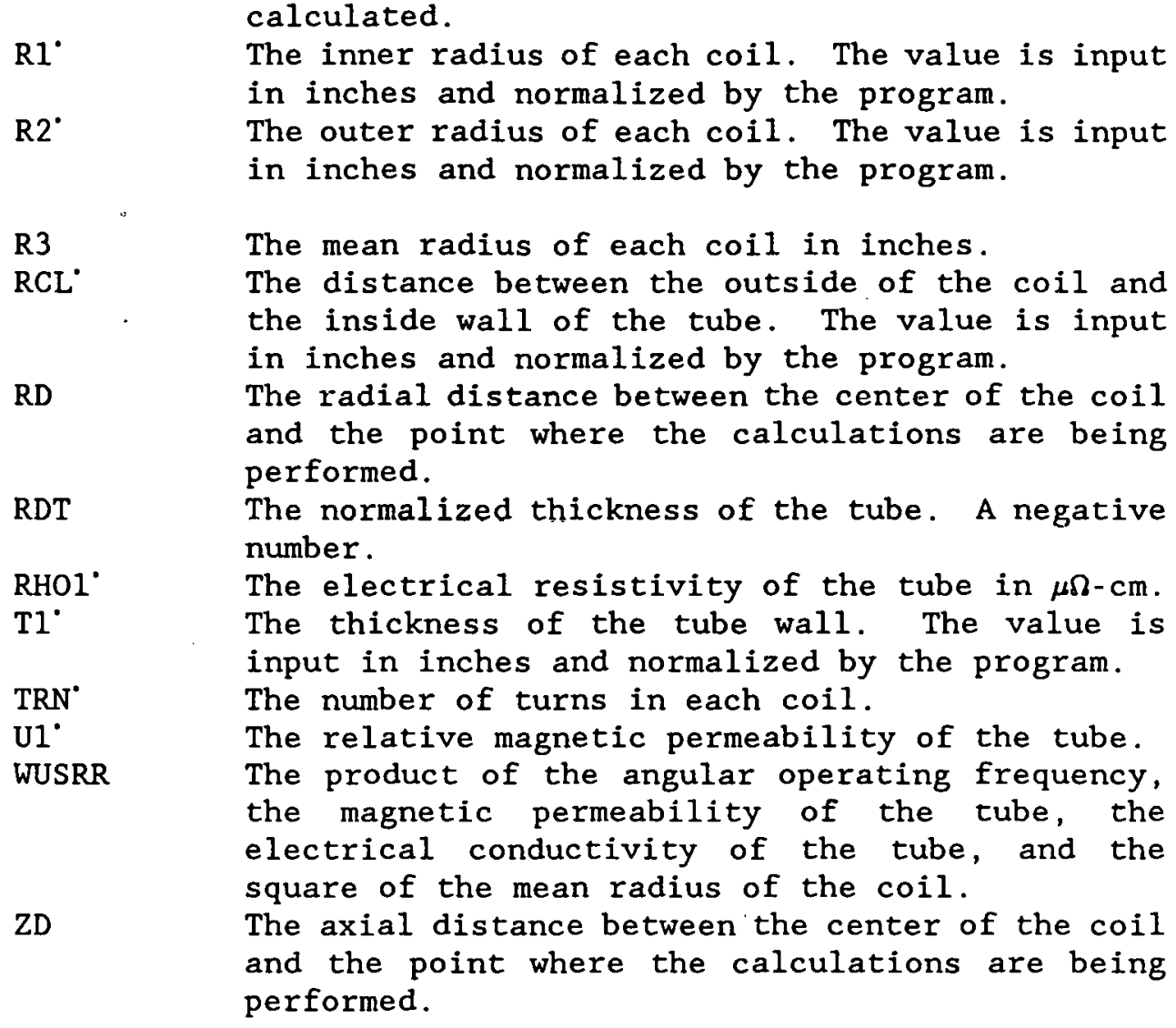

Sample output

A listing of the printer output is shown below:

\begin{tabular}{|c|c|c|c|c|c|c|c|c|}
\hline BDSF & TIM & $9: 59$ & DATE & $8 / 16 / 89$ & & & & \\
\hline & IN RAD & OT RAD & LENGTH & RAD CLR & WALLTH & C TO C & TUB IR & TUB OR \\
\hline CT & 1.2400 & 1.4900 & 0.2650 & 0.0575 & 0.2200 & 0.5150 & 1.5475 & 1.7675 \\
\hline OR & 0.9084 & 1.0916 & 0.1941 & 0.0421 & 0.1612 & 0.3773 & 1.1337 & 1. 2949 \\
\hline
\end{tabular}

RBAR $1.3650 \mathrm{FREQ}=4.000000 \mathrm{E}+02 \mathrm{RHO}=3.8400 \mathrm{PERM}=1.000 \mathrm{WUSRR}=98.8670$

A partial listing of the file FORT40 is given below:

$50 \quad 40$

$\begin{array}{ll}0.02500 & 0.00403 \\ 0.04212 & \\ 0.90842 & 1.09158 \\ 0.37729 & 0.19414 \\ 1.13370 & 1.29487\end{array}$

$0 \begin{array}{cccc}0 & 0 & 0.00000 \mathrm{D}+00 & 0.00000 \mathrm{D}+00\end{array}$

$\begin{array}{llll}0 & 1 & 0.00000 \mathrm{D}+00 & 0.00000 \mathrm{D}+00\end{array}$

$\ldots \ldots \ldots$ (zero for all of first row)....

$\begin{array}{llll}1 & 0 & 0.24266 \mathrm{D}-01 & 0.33487 \mathrm{D}+00\end{array}$

$\begin{array}{llll}1 & 1 & 0.25157 \mathrm{D}-01 & 0.33978 \mathrm{D}+00\end{array}$

$12 \quad 2 \quad 0.26086 \mathrm{D}-01 \quad 0.34774 \mathrm{D}+00$

$130.27053 \mathrm{D}-01 \quad 0.35864 \mathrm{D}+00$ 
$144 \quad 0.28061 D-01 \quad 0.37238 D+00$

$\begin{array}{lllll}1 & 5 & 0.29113 \mathrm{D}-01 & 0.38887 \mathrm{D}+00\end{array}$

$\begin{array}{llll}1 & 6 & 0.30213 \mathrm{D}-01 & 0.40800 \mathrm{D}+00\end{array}$

$\begin{array}{llll}1 & 7 & 0.31364 \mathrm{D}-01 & 0.42968 \mathrm{D}+00\end{array}$

$\begin{array}{llll}1 & 8 & 0.32570 \mathrm{D}-01 & 0.45382 \mathrm{D}+00\end{array}$

$\begin{array}{llll}1 & 9 & 0.33836 \mathrm{D}-01 & 0.48031 \mathrm{D}+00\end{array}$

$1 \quad 10 \quad 0.35166 \mathrm{D}-01 \quad 0.50906 \mathrm{D}+00$ 


\section{Listing}

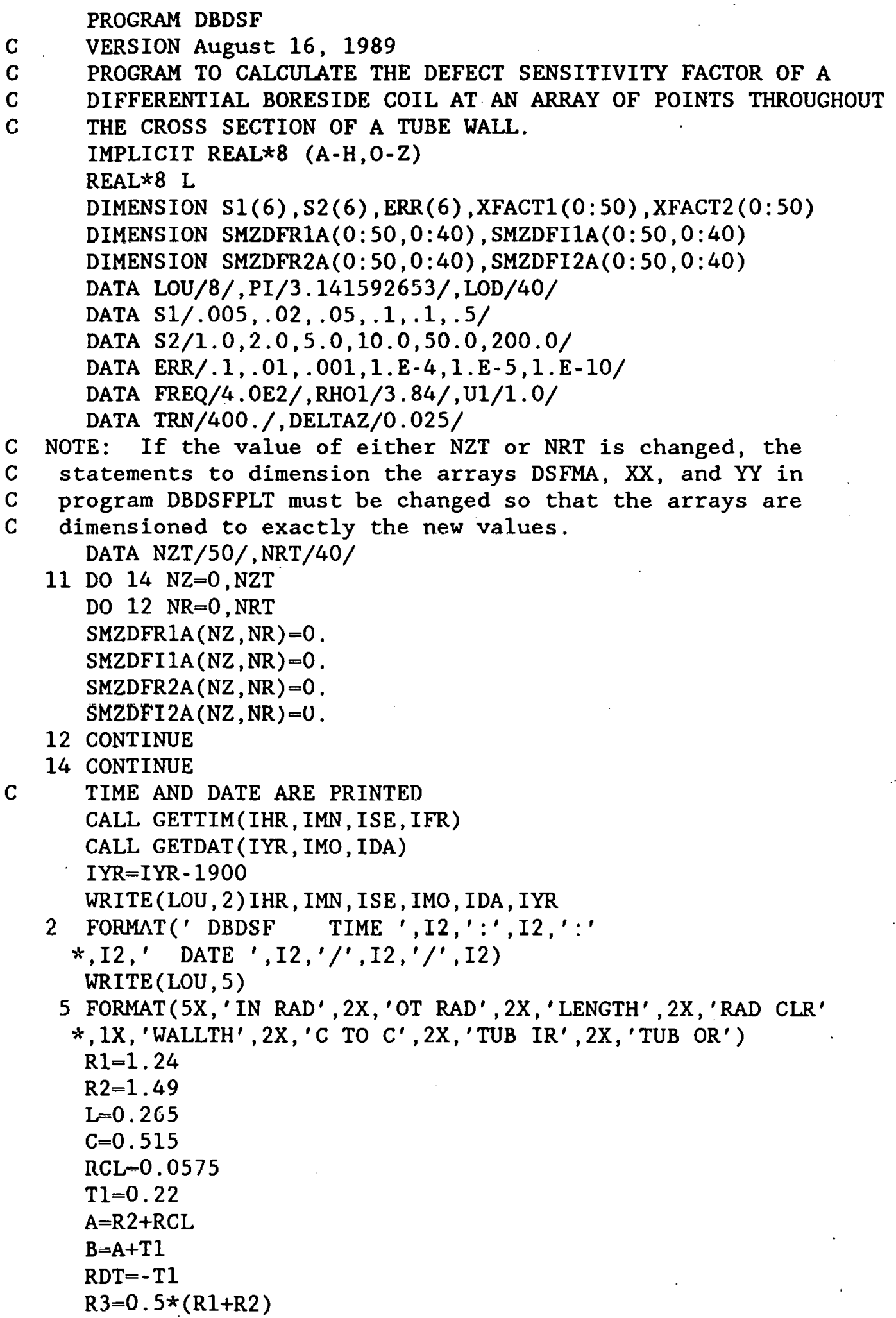




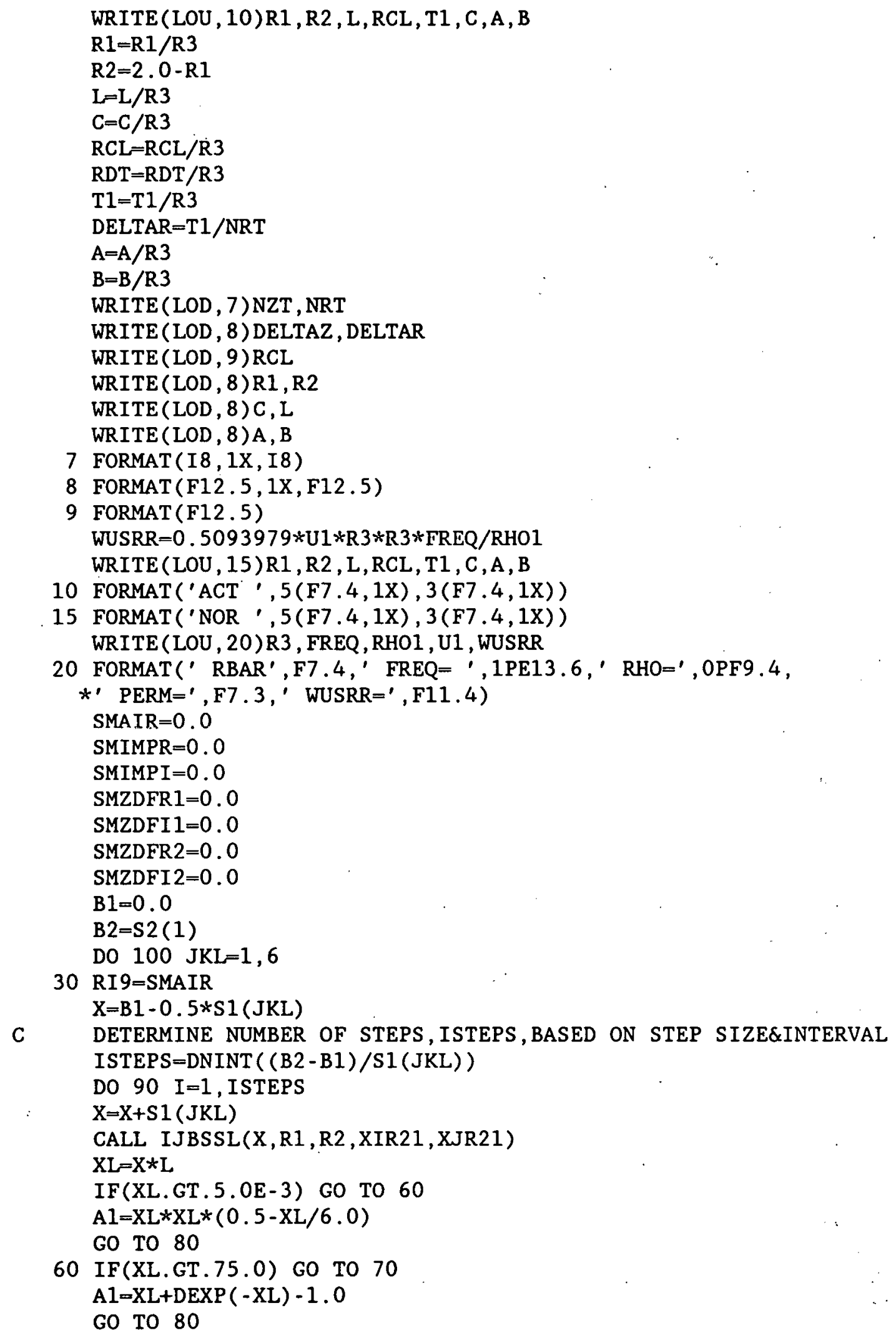


$70 \quad \mathrm{Al}=\mathrm{XL}-1.0$

80 SMAIR $=$ SMAIR $+2 *$ XJR $21 * X J R 21 * A 1 * S 1(J K L)$

IF (X.GT.160.) GO TO 90

$\mathrm{XX}=\mathrm{X} * \mathrm{X}$

$\mathrm{XXXX}=\mathrm{XX} * \mathrm{XX}$

$\mathrm{X} 1-\mathrm{DSQRT}(0.5 *(\mathrm{XX}+\mathrm{DSQRT}(\mathrm{XXXX}+\mathrm{WUSRR} *$ WUSRR $))) / \mathrm{U} 1$

$\mathrm{Y} 1=\mathrm{WUSRR} /(2 * \mathrm{X} 1 * \mathrm{U} 1 * \mathrm{U} 1)$

$X A=X * A$

$\mathrm{XB}=\mathrm{X} * \mathrm{~B}$

$\mathrm{X} 1 \mathrm{~A}=\mathrm{X} 1 * \mathrm{~A}$

$\mathrm{Y} 1 \mathrm{~A}=\mathrm{Y} 1 * \mathrm{~A}$

$\mathrm{X} 1 \mathrm{~B}=\mathrm{X} 1 * \mathrm{~B}$

$\mathrm{Y} 1 \mathrm{~B}=\mathrm{Y} 1 * \mathrm{~B}$

CALL CMDBES (X1A, Y1A, ZIORA, ZIOIA, ZKORA, ZKOIA, BI1RA, BIIIA

*, BK1RA, BK1IA)

CALL CMDBES (X1B, Y1B , ZIORB , ZIOIB , ZKORB , ZKOIB , BIIRB , BIIIB

*, BKIRB , BK1IB)

CALL BESI (XA, BIOA, BI1A)

CALL BESK(XA, BKOA, BK1A)

CALL BESI (XB, BIOB, BI1B)

CALL BESK(XB, BKOB, BK1B)

$\mathrm{DR} 1-\mathrm{XB} * \mathrm{BKOB} * \mathrm{BK} 1 \mathrm{RB}-\mathrm{ZKORB} * \mathrm{BK} 1 \mathrm{~B} / \mathrm{U} 1$

$\mathrm{DI} 1=\mathrm{XB} * \mathrm{BKOB} * \mathrm{BK} 1 \mathrm{IB}-\mathrm{ZKOIB} * \mathrm{BK} 1 \mathrm{~B} / \mathrm{U} 1$

$\mathrm{DR} 2=\mathrm{ZIORA} * \mathrm{BI} 1 \mathrm{~A} / \mathrm{U} 1-\mathrm{XA} * \mathrm{BIOA} * \mathrm{BI} 1 \mathrm{RA}$

DI2 $=Z I O I A * B I 1 A / U 1-X A * B I O A * B I I I A$

$\mathrm{DR} 3=\mathrm{XA} * \mathrm{BIOA} * \mathrm{BK} 1 \mathrm{RA}+\mathrm{ZKORA} * \mathrm{BI} 1 \mathrm{~A} / \mathrm{U} 1$

$\mathrm{DI} 3=\mathrm{XA} * \mathrm{BIOA} * \mathrm{BK} 1 \mathrm{IA}+\mathrm{ZKOIA} * \mathrm{BI} 1 \mathrm{~A} / \mathrm{U} 1$

$\mathrm{DR} 4=\mathrm{ZIORB} * \mathrm{BK} 1 \mathrm{~B} / \mathrm{U} 1+\mathrm{XB} * \mathrm{BKOB} * \mathrm{BI} 1 \mathrm{RB}$

$\mathrm{DI} 4=\mathrm{ZIOIB} * \mathrm{BK} 1 \mathrm{~B} / \mathrm{U} 1+\mathrm{XB} * \mathrm{BKOB} * \mathrm{BIIIB}$

$\mathrm{DDR}=\mathrm{DR} 1 * \mathrm{DR} 2-\mathrm{DI} 1 * \mathrm{DI} 2+\mathrm{DR} 3 * \mathrm{DR} 4-\mathrm{DI} 3 * \mathrm{DI} 4$

$\mathrm{DDI}=\mathrm{DR} 1 * \mathrm{DI} 2+\mathrm{DR} 2 * \mathrm{DI} 1+\mathrm{DR} 3 * \mathrm{DI} 4+\mathrm{DR} 4 * \mathrm{DI} 3$

$S N R=-B I 1 R A * D R 1+B I 1 I A * D I 1+B K 1 R A * D R 4-B K 1 I A * D I 4$

$S N I=-B I 1 I A * D R 1-B I 1 R A * D I 1+B K 1 R A * D I 4+B K 1 I A * D R 4$

$\mathrm{DEN}=\mathrm{DDR} * \mathrm{DDR}+\mathrm{DDI} * \mathrm{DDI}$

$S S R=((S N R * D D R+S N I * D D I) / D E N-B K 1 A) / B I I A$

$S S I=(S N I * D D R-S N R * D D I) /(B I 1 A * D E N)$

$\mathrm{XFACT}=\mathrm{XIR} 21 * \mathrm{DSIN}(\mathrm{XL} / 2$.

SMIMPR $=S M I M P R+8 . * \mathrm{XFACT} * \mathrm{XFACT} * \mathrm{~S} 1(\mathrm{JKL}) * \mathrm{SSR} / \mathrm{PI}$

SMIMPI $=$ SMIMPI +8 * *XFACT *XFACT $* S 1(\mathrm{JKL}) * \mathrm{SSI} / \mathrm{PI}$

$\mathrm{XF}=4$. $* \mathrm{XFACT} * \mathrm{~S} 1(\mathrm{JKL}) / \mathrm{PI}$

$\mathrm{XF} 1=\mathrm{XF} * \mathrm{DSIN}(0.5 * \mathrm{X} * \mathrm{C})$

$\mathrm{XF} 2=\mathrm{XF} * \mathrm{DCOS}(0.5 * \mathrm{X} * \mathrm{C})$

DO $89 \mathrm{NR}=0$, NRT

$\operatorname{RD}-(\operatorname{REAL}(\mathrm{NR})) *(\mathrm{RDT} / \mathrm{REAL}(\mathrm{NRT}))$

$\mathrm{RD}=\mathrm{B}+\mathrm{RD}$

$\mathrm{X} 1 \mathrm{R}=\mathrm{X} 1 * \mathrm{RD}$

$\mathrm{Y} 1 \mathrm{R}=\mathrm{Y} 1 * \mathrm{RD}$

CALL CMDBES (X1R, Y1R, ZIORR, ZIOIR, ZKORR, ZKOIR, BIIRR, BIIIR $*, B K 1 R R, B K 1 I R$ ) 
$\mathrm{DNR}=\mathrm{BK} 1 \mathrm{RR} * \mathrm{DR} 4-\mathrm{BK} 1 \mathrm{IR} * \mathrm{DI} 4-\mathrm{BI} 1 \mathrm{RR} * \mathrm{DR} 1+\mathrm{BI} 1 \mathrm{IR} * \mathrm{DI} 1$

$\mathrm{DNI}=\mathrm{BK} 1 \mathrm{IR} * \mathrm{DR} 4+\mathrm{BK} 1 \mathrm{RR} * \mathrm{DI} 4-\mathrm{BI} 1 \mathrm{IR} * \mathrm{DR} 1-\mathrm{BI} 1 \mathrm{RR} * \mathrm{DI} 1$

$\mathrm{DFR}=(\mathrm{DNR} * \mathrm{DDR}+\mathrm{DNI} * \mathrm{DDI}) / \mathrm{DEN}$

$\mathrm{DFI}=(\mathrm{DNI} * \mathrm{DDR}-\mathrm{DNR} * \mathrm{DDI}) / \mathrm{DEN}$

DO $88 \mathrm{NZ}=0$, NZT

IF(NR.GT.1)GO TO 87

$\mathrm{ZD}=\mathrm{DELTAZ} * \mathrm{NZ}$

$\mathrm{XFACT} 1(\mathrm{NZ})=\mathrm{XF} 1 * \mathrm{DSIN}(\mathrm{X} * \mathrm{ZD})$

$\mathrm{XFACT} 2(\mathrm{NZ})=\mathrm{XF} 2 * \mathrm{DCOS}(\mathrm{X} * \mathrm{ZD})$

87 SMZDFR1A (NZ, NR) $=$ SMZDFR1A (NZ, NR) +XFACT1 (NZ) *DFR

SMZDFI1A (NZ, NR) $=$ SMZDFI IA (NZ, NR) +XFACT1 (NZ) *DFI

SMZDFR2A (NZ, NR) $=$ SMZDFR2A (NZ, NR) +XFACT2 (NZ) *DFR

88 CONTINUE

SMZDFI2A (NZ, NR) $=$ SMZDFI $2 A(N Z, N R)+X F A C T 2(N Z) * D F I$

89 CONTINUE

90 CONTINUE

$\mathrm{B} 1=\mathrm{B} 2$

$\mathrm{B} 2=\mathrm{B} 2+\mathrm{S} 2$ (JKL)

CHECK $=($ SMAIR - RI9) $/$ SMAIR

IF (ABS (CHECK) . GT . ERR (JKL)) GO TO 30

100 CONTINUE

DO $990 \mathrm{NZ}=0$, NZT

$\mathrm{ZD}=\mathrm{DELTAZ} * \mathrm{NZ}$

SMZDFR $1=0$.

SMZDFI $1=0$.

SMZDFR2 $=0$.

SMZDFI $2=0$.

DO $120 \mathrm{NR}=0$, NRT

SMZDFR1=SMZDFR1A (NZ, NR)

SMZDFI $1=$ SMZDFI $1 A(N Z, N R)$

SMZDFR2 $=$ SMZDFR2A $(\mathrm{NZ}, \mathrm{NR})$.

SMZDFI2 $=$ SMZDFI $2 A(N Z, N R)$

DSFR $=-1.5 *$ WUSRR * (SMZDFR $1 *$ SMZDFR2 - SMZDFI $1 *$ SMZDFI 2$) /($ SMAIR*PI $)$

$\mathrm{DSFI}=-1.5 *$ WUSRR * (SMZDFR $1 *$ SMZDFI $2+\mathrm{SMZDFR} 2 * \operatorname{SMZDFI} 1) /(\mathrm{SMAIR} * \mathrm{PI})$

$\mathrm{DSFM}=\mathrm{DSQRT}(\mathrm{DSFR} * \mathrm{DSFR}+\mathrm{DSFI} * \mathrm{DSFI})$

DSFP $=$ DATAN2 (DSFI, DSFR)

WRITE (LOD , 126) NZ, NR, DSFM, DSFP

c

WRITE $(0,126) \mathrm{NZ}, \mathrm{NR}, \mathrm{DSFM}, \mathrm{DSFP}$

120 CONTINUE

126 FORMAT (I5 , 1X, I5 , 1X, D12.5, 1X, D12.5)

990 CONTINUE

1000 CONTINUE

1001 END 
DBDSFPLT generates a contour plot from DBDSF data

Program DBDSFPLT generates a contour plot of the magnitude of the defect sensitivity factor for a differential boreside coil using calculations performed and stored by the program DBDSF in the file FORT 40.

\section{Summary}

1. Dimension arrays and declare variable types.

2. Initialize vartables.

3. Open the file created by program DBDSF.

4. Read in the information about the coil and tube from the file.

5. Calculate the position of the data points in the normalized coordinate system.

6. Set the label flags for the contours.

7. Read the data stored by program DBDSF into array DSFMA.

8. Specify the values of the magnitude of the defect sensitivity factor where the contours are to be drawn.

9. Call the PRINTMATIC contour initialization routines.

10. Draw the contours .

11. Draw the coil and tube.

\section{Variables}

\begin{tabular}{|c|c|}
\hline A & The normalized inner radius of the tube. \\
\hline B & The normalized outer radius of the tube. \\
\hline C & The center-to-center spacing between the coils. \\
\hline $\mathrm{CNM}^{\circ}$ & $\begin{array}{l}\text { Array giving the values of the magnitude of the } \\
\text { defect sensitivity factor at which contours are } \\
\text { to be drawn. }\end{array}$ \\
\hline DELTAR & $\begin{array}{l}\text { The normalized distance in the radial direction } \\
\text { between adjacent data points. }\end{array}$ \\
\hline DELTAZ & $\begin{array}{l}\text { The normalized distance in the axial direction } \\
\text { between adjacent data points. }\end{array}$ \\
\hline DSFMA & $\begin{array}{l}\text { Array containing the values of the magnitude of } \\
\text { the defecl seisitivity factor which were read in } \\
\text { from the data file. }\end{array}$ \\
\hline L & The normalized length of the coil. \\
\hline LBM $^{\circ}$ & $\begin{array}{l}\text { Array which tells the program which of the } \\
\text { contours are to be labeled with their values. If } \\
\text { all elements of LBM are zero, none of the contours } \\
\text { will be labeled. }\end{array}$ \\
\hline LOE* & $\begin{array}{l}\text { lhe number of the } I / 0 \text { unit connected to the input } \\
\text { data file. }\end{array}$ \\
\hline NAME & $\begin{array}{l}\text { Character variable which contains the name of the } \\
\text { file which this program uses for output. }\end{array}$ \\
\hline NC* & The number of contours to be drawn. \\
\hline NRT & $\begin{array}{l}\text { The total number of points in the radial direction } \\
\text { at which calculations were performed. }\end{array}$ \\
\hline $\mathrm{ZT}$ & he total number of points in the axial direction \\
\hline
\end{tabular}


R1 at which calculations were performed.

R2 The normalized outer coil radius.

RCL The normalized distance between the outside of the

$\mathrm{XX}$ Array describing the axial location of the data

YY Array describing the radial location of the data points in the normalized coordinate system.

\section{Notes}

1. Program DBDSFPLT does not actually send anything to the printer; it merely creates a file whose name is given by the program variable NAME. If the value of NAME is 'filename.ext', then to print the file created by program DBDSFPLT, enter

DPRINT filename.ext

DPRINT.EXE is a program supplied by PRINTMATIC. For this particular program the variable NAME is set to PCDSF.FIL so to make a plot one would type:

DPRINT DBDSF.FIL

\section{Sample output}

We show a plot of the data from DBDSF plotted using DBDSFPLT in Fig. 16. The phase contours can be plotted rather than the magnitude by using the second statement with label 140 and commenting the first one out. 


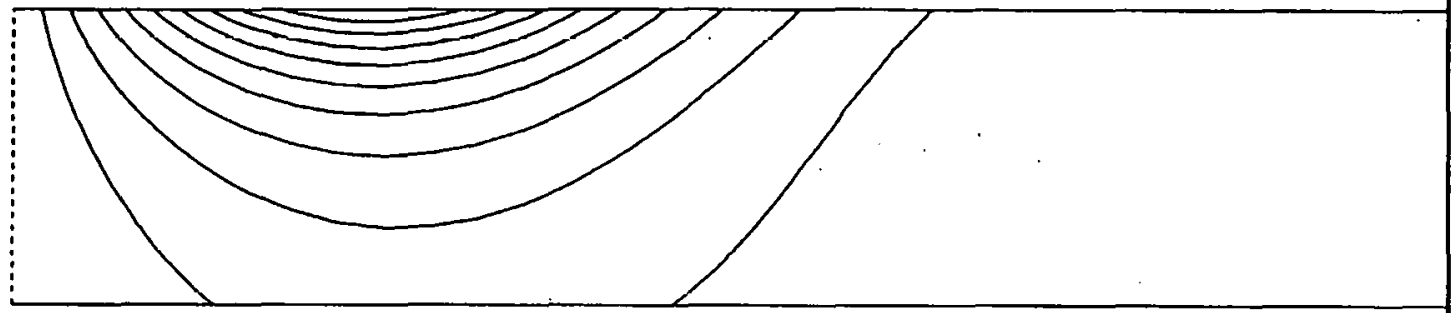

Fig. 16. Contour plot of the magnitude of the defect sensitivity factor for a differential boreside probe.

\section{Listing}

\section{PROGRAM DBDSFPLT}

C VERSION October 25,1988

C Program to generate a contour plot of the magnitude of the

$C$ defect sensitivity factor of a differential boreside coil.

C

CHARACTER $* 80$ NAME

IMPLICIT REAL*4 (A-H,O-Z)

REAL*4 DSFMA $(51,41)$

REAL*4 XX(51), YY(41)

REAL*4 CNM(10)

REAL*4 L

INTEGER *2 LBM(10)

INTEGER $* 2 \mathrm{I} 1, \mathrm{~J} 1, \mathrm{I} 2, \mathrm{~J} 2$

DATA XSCALE $/ 1.0 /, \mathrm{NC} / 9 /$

DATA IDEF/2/,LOE/40/

C

Open the file created by program DBDSF and read in the coil and tube information.

C

OPEN (LOE , FILE=' FORT40', STATUS=' OLD' $)$

$\operatorname{READ}(\mathrm{LOE}, *)$ NZT , NRT 


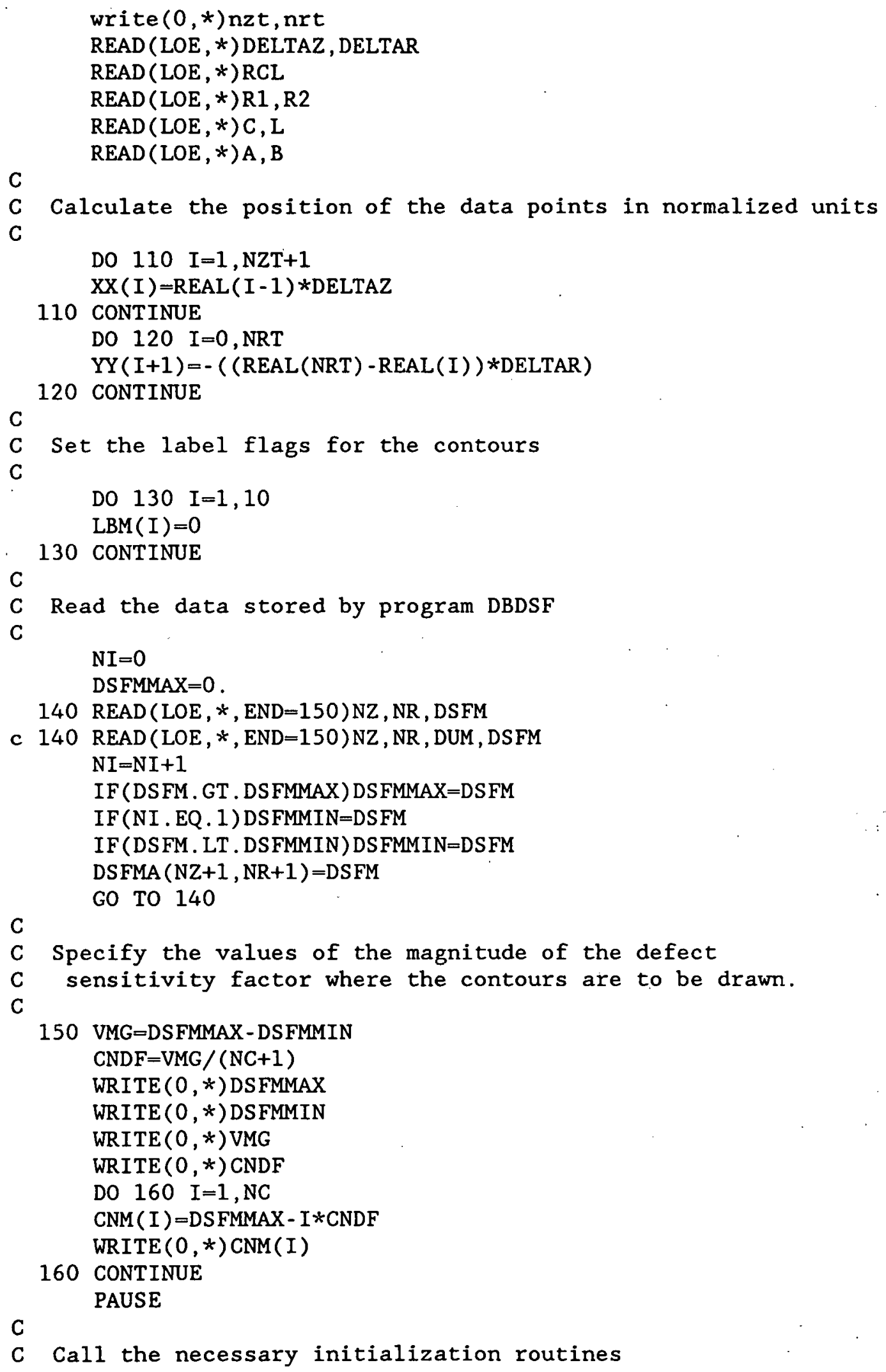

C Call the necessary initialization routines 
C

NAME $=$ ' DBDSF. FIL'

CALL DINIT (NAME)

CALL DPLOT $(1 ., 1 ., 8,4 ., 0 ., 0.8,-0.2,0.2,0 ., 0$.

C

CALL DCTRDEF $(1,1,1,1,1)$

C Draw the contours

C

$n z t=n z t+1$

$n r t=n r t+1$

C

CALL DCNTOUR (XSCALE, XX, YY, DSFMA, CNM , LBM, NZT , NRT , NC, IDEF)

C Draw the tube

C

$\mathrm{X} 1=0$.

$\mathrm{Y} 1=0$.

$\mathrm{X} 2=0.8$

$\mathrm{Y} 2=-(\mathrm{B}-\mathrm{A})$

write $(0, *) \mathrm{j} 2$

CALL DRTOI. $(\mathrm{X} 1, \mathrm{Y} 1, \mathrm{I} 1, \mathrm{~J} 1)$

CALL DRTOI (X2, Y2, I 2, J 2)

write $(0, *) I 1, J 1, I 2, J 1$

CALL DLINE(I1, J1, I $2, \mathrm{~J} 1$ )

write $(0, *) \mathrm{I} 1, \mathrm{~J} 1, \mathrm{I} 2, \mathrm{~J} 1$

CALL DLINE(I1, J2, I $2, \mathrm{~J} 2)$

$\mathrm{C}$

write $(0, *) \mathrm{j} 2$

C Draw the coil

C

$\mathrm{X} 1=0.5 *(\mathrm{C}-\mathrm{L})$

$\mathrm{Y} 1=\mathrm{RCL}$

$\mathrm{X} 2=0.5 *(\mathrm{C}+\mathrm{L})$

$\mathrm{Y} 2=\mathrm{RCL}+\mathrm{R} 2-\mathrm{R} 1$

CALL DRTOI (X1, Y1, I1, J1)

CALL DRTOI $(X 2, Y 2, I 2, \mathrm{~J} 2)$

write $(0, *) j 2$

CALL DLINE(I1,J1, I $2, \mathrm{~J} 1$ )

CALL DLINE(I1, J2, I2, J2)

CALL DLINE(I1,J1, I1, J2)

write $(0, *) \mathrm{I} 2, \mathrm{~J} 1, \mathrm{I} 2, \mathrm{~J} 2$

CALL DLINE(I2,J1, I2, J2)

write $(0, *) \mathrm{I} 2, \mathrm{~J} 1, \mathrm{I} 2, \mathrm{~J} 2$

C

C Draw a dotted line on the plane between the coils

C

$\mathrm{X} 1=0$.

$\mathrm{Y} 1=0$.

$\mathrm{X} 2=0$.

$\mathrm{Y} 2=-(\mathrm{B}-\mathrm{A})$

CALL DRTOI $(X 1, \mathrm{Y} 1, \mathrm{I} 1, \mathrm{~J} 1)$

CALL DRTOI (X2, Y2,I2,J2) 
CALL DDASH(I $1, \mathrm{~J} 1, \mathrm{I} 2, \mathrm{~J} 2,1,10,10)$

CALL DFINIS

write $(0, *) \mathrm{j} 2$

STOP

END 
DFBORAR calculates defect impedance change, average over depth

Program DFBORAR calculates the change in the normalized impedance of a differential boreside coil due to the presence of a defect in a single conducting tube, as shown in Fig. 15. The equations computed are the same as those for the defect sensitivity factor, with the defect volume included. The distance to the defect is measured from the center of the coil assembly, and the center-to-center distance of the coils is denoted c. The impedance difference between the two matched coils is:

$$
Z_{\text {nd }}=Z_{1 d}-Z_{2 d}
$$

Substituting in from Eq. (29) for the impedance change of each coil and using some trigonometric identities gives for the normalized impedance difference :

$$
\begin{aligned}
Z_{\mathrm{nd}}(r, z)=\frac{-3\left(\omega \mu \sigma_{1} \overline{\mathrm{r}}^{2}\right) V o I_{\mathrm{n}}}{2 \pi I_{\mathrm{a} \mid \mathrm{r}}} \\
\times\left[\int_{0}^{\infty} \frac{I\left(r_{2}, r_{1}\right)}{\pi \alpha^{3}}\left\{\frac{K_{1}\left(\alpha_{1} \mathrm{r}\right) D_{4}-I_{1}\left(\alpha_{1} \mathrm{r}\right) D_{1}}{\left(D_{1} D_{2}+D_{3} D_{4}\right)}\right\} \sin \left(\frac{\alpha \ell}{2}\right) \sin \left(\frac{\alpha c}{2}\right) \sin (\alpha z) 4 d \alpha\right] \\
\times\left[\int_{0}^{\infty} \frac{I\left(r_{2}, r_{1}\right)}{\pi \alpha^{3}}\left\{\frac{K_{1}\left(\alpha_{1} \mathrm{r}\right) D_{4}-I_{1}\left(\alpha_{1} \mathrm{r}\right) D_{1}}{\left(D_{1} D_{2}+D_{3} D_{4}\right)}\right\} \sin \left(\frac{\alpha \ell}{2}\right) \cos \left(\frac{\alpha c}{2}\right) \cos (\alpha z) 4 d \alpha\right]
\end{aligned}
$$

The definition of the various terms in Eq. (42) are given in Eqs. (30) through (38) in the discussion of the absolute boreside coil. It performs the calculations for a number of different axial distances between the center of the coil and the defect, and it can divide the defect into a number of parts and calculate the effect of each part separately. The effect of the defect is averaged over the depth of the defect, and the defect is moved from the probe center $(z=0)$ outward in the positive $z$ direction.

\section{$\underline{\text { Summary }}$}

1. Dimension arrays and declare variable types.

2. Initialize variables.

3. Begin the integration. Calculate the expressions that are independent of the position of the defect.

4. Select a value for the radial distance between the defect and the center of the coil. Do the calculations which depend only upon this component of the position.

5. Select a value for the axial distance between the defect and the center of the coil. Complete the calculations.

6. Loop to 5 until done.

7. Loop to 4 until done. 
8. Output the results.

\section{Variables}

A number of the variables which occur mainly inside the integration loops do not correspond to anything physical. These variables are described in a section at the end. Starred variables must be assigned by the user.

A
B
C

DELTAZ

DFDEP'

DFDIAM*

DFM

DFP

DSFI

DSFR

FREQ

ISIDE*

$\mathrm{E}^{*}$

LOD $^{*}$

LOE'

LOU

NRT*

N:Z:"

POW

Q6

R1"
The normalized inner radius of the tube.

The normalized outer radius of the tube.

The axial distance between the centers of the two coils. The value is input in inches and normalized by the program.

The normalized axial distance between the points at which the calculations are done.

The depth of the defect in the tube in inches.

The diameter of the defect in the tube in inches. The magnitude of the normalized impedance change in the coil due to the defect.

The phase of the normalized impedance change in the coil due to the defect.

The imaginary part of the defect sensitivity factor of the coil.

The real part of the defect sensitivity factor of the coil.

The operating frequency in hertz.

The side of the tube where the defect is located. If ISIDE $=1$, the defect is on the outside of the tube; if $\operatorname{ISIDE}=2$, the defect is on the inside of the tube.

The length of each coil. The value is input in inches and normalized by the program.

The number of the $I / O$ unit connected to the output data file.

The number of the $I / O$ unit connected to a file which contains the diameters and depths of the defects for which calculations are to be performed.

The number of the $I / O$ unit connected to the printer.

The total number of parts centered along the axis of the defect into which the defect is divided to perform the calculations.

The total number of different values of the axial distance between the center of the coil and the defect for which calculations are performed.

The depth of the defect expressed as a percentage of wall thickness.

The inductance in henries of the coil in air.

The inner radius of each coil. The value is input in inches and normalized by the program. 

R2 $^{\circ} \quad$ The outer radius of each coil. The value is input in inches and normalized by the program.
R3 The mean radius of each coil in inches.
$\mathrm{RCL}^{\circ} \quad$ The distance between the outside of the coil and the inside wall of the tube. The value is input in inches and normalized by the program.
$\mathrm{RD}$ The radial distance between the center of the coil and the defect (see note 1).
RDT The normalized depth to the bottom of the defect. A negative number.
$\mathrm{RHO1}^{\circ}$ The electrical resistivity of the tube in $\mu \Omega-\mathrm{cm}$. T1 The thickness of the tube wall. The value is input in inches and normalized by the program.
TRN" The number of turns in each coil.
U1 The relative magnetic permeability of the tube.
VOLN The normalized volume of the defect.
WUSRR The product of the angular operating frequency, the magnetic permeability of the tube, the electrical conductivity of the tube, and the square of the mean radius of the coil.
ZD The axial distance between the center of the coil and the defect.
ZNDFI The imaginary part of the normalized impedance change in the coil due to the defect.
ZNDFR The real part of the normalized impedance change in the coil due to the defect.
ZNIM The imaginary part of the normalized impedance of the coil when no defects are present.
ZNRL The real part of the normalized impedance of the coil when no defects are present.

\section{Notes}

1. The program variable $R D$ does not give the radial distance between the actual defect and the center of the coil; it gives the radial distance between the part of the defect with which the program is working at any ine and lite center of the coil. 
Integration Section of Program DFBORAR

\section{Symbol definitions}

The following are definitions of the symbols used to describe the program variables which appear in the integration section of the program. All lengths are normalized unless otherwise noted.

$\alpha$
$a$
$\beta_{1}$
$b$
$c$
$I\left(x_{2}, x_{1}\right)$
$I_{0}(x)$
$I_{1}(x)$
$J\left(x_{2}, x_{1}\right)$
$J_{1}(x)$
$K_{0}(x)$
$K_{1}(x)$
$\ell$
$\mu$
$r$
$r$
$r_{1}$
$r_{2}$
$\sigma$
$t_{1}$
$z$
$\omega$

Integration variable

Inner radius of the tube $\left(\alpha^{2}+j \omega \mu \sigma, \bar{r}^{2}\right)^{1 / 2} / \mu$

Outer radius of the tube

Distance between the coil centers

Integral of $x I_{1}(x)$ with respect to $x$ from $\alpha x_{1}$ to $\alpha x_{2}$ Modified Bessel function of the first kind of order 0 Modified Bessel function of the first kind of order 1 Integral of $x J_{1}(x)$ with respect to $x$ from $\alpha x_{1}$ to $\alpha x_{2}$ Bessel function of the first kind of order 1 Modified Bessel function of the second kind of order 0 Modified Bessel function of the second kind of order 1 Length of the coill

Relative magnetic permeability of the tube

Radial distance between center of coil and defect

Coil mean radius in inches

Inner radius of coil

Outer radius of coil

Electrical conductivity of the tube

Thickness of the tube

Axial distance between defect and probe center

Angular operating frequency

\section{Variables appearing in the integration section}

\begin{tabular}{|c|c|}
\hline $\begin{array}{l}\text { Program } \\
\text { variable }\end{array}$ & $\begin{array}{l}\text { Symbolic } \\
\text { equivalent }\end{array}$ \\
\hline$\overline{A 1}$ & $\overline{\alpha \ell+\exp (-\alpha \ell)-1}$ \\
\hline BIOA & $I_{0}(\alpha a)$ \\
\hline $\mathrm{BIOB}$ & $I_{0}(\alpha b)$ \\
\hline BIIA & $I_{1}(\infty a)$ \\
\hline $\mathrm{BI} 1 \mathrm{~B}$ & $I_{1}(\alpha b)$ \\
\hline BI1IA & $\operatorname{Im}\left[I_{1}(\alpha, a)\right]$ \\
\hline BIIIB & $\operatorname{Im}\left[I_{1}(\alpha, b)\right]$ \\
\hline BIIIR & $\operatorname{Im}\left[I_{1}(\alpha, r)\right]$ \\
\hline BIIRA & $\operatorname{Re}\left[I_{1}\left(\alpha_{1} a\right)\right]$ \\
\hline BIIRB & $\operatorname{Re}\left[I_{1}\left(\alpha_{1} b\right)\right]$ \\
\hline
\end{tabular}


BIIRR

BK.OA

BKOB

BKIA

BK1B

BK1 IA

BKIIB

BK1IR

BKIRA

BKIRB

BKIRR

DDI

DDR

DFR

DI1

DI2

DI 3

DI4

DNI
$\operatorname{Re}\left[I_{1}(\alpha, r)\right]$

$K_{0}(\alpha a)$

$K_{0}(\alpha b)$

$K_{1}(\alpha a)$

$K_{1}(\alpha b)$

$\operatorname{Im}\left[K_{1}(\alpha, a)\right]$

$\operatorname{Im}\left[K_{1}(\alpha, b)\right]$

$\operatorname{Im}\left[K_{1}(\alpha, r)\right]$

$\operatorname{Re}[K,(\alpha, a)]$

$\operatorname{Re}[K,(\alpha, b)]$

$\operatorname{Re}\left[K_{1}(\alpha, r)\right]$

$\operatorname{Im}\left\{\left[\beta_{2} b K_{0}\left(\alpha_{2} b\right) K_{1}\left(\alpha_{1} b\right)-\beta_{1} b K_{0}\left(\alpha_{1} b\right) K_{1}\left(\alpha_{2} b\right)\right]\right.$

$\left[\beta, a I_{0}(\alpha, a) I_{1}(\alpha a)-\alpha a I_{0}(\alpha a) I_{1}(\alpha, a)\right]+$

$\left[\alpha a I_{0}(\alpha a) K_{1}(\alpha, a)+\beta_{1} a K_{0}(\alpha, a) I_{1}(\alpha a)\right]$

$\left.\left[\beta, b I_{0}(\alpha, b) K_{1}\left(\alpha_{2} b\right)+\beta_{2} b K_{0}\left(\alpha_{2} b\right) I_{1}(\alpha, b)\right]\right\}$

$\operatorname{Re}\left[\left[\beta_{2} b K_{0}\left(\alpha_{2} b\right) K_{1}\left(\alpha_{1} b\right)-\beta_{1} b K_{0}\left(\alpha_{1} b\right) K_{1}\left(\alpha_{2} b\right)\right]\right.$

$\left[\beta, a I_{0}(\alpha, a) I_{1}(\alpha a)-\alpha a I_{0}(\alpha a) I_{1}(\alpha, a)\right]+$

$\left[\alpha a I_{0}(\alpha a) K_{1}(\alpha, a)+\beta_{1} a K_{0}(\alpha, a) I_{1}(\alpha a)\right]$

$\left.\left[\beta, b I_{0}\left(\alpha_{1} b\right) K_{1}\left(\alpha_{2} b\right)+\beta_{2} b K_{0}\left(\alpha_{2} b\right) I_{1}\left(\alpha_{1} b\right)\right]\right\}$

$\operatorname{Re}\left\{K_{1}(\alpha, r)\left[\beta, b I_{0}(\alpha, b) K_{1}\left(\alpha_{2} b\right)+\beta_{2} b K_{0}\left(\alpha_{2} b\right) I_{1}(\alpha, b)\right]-\right.$

$$
\left.I_{1}(\alpha, r)\left[\beta_{2} b K_{0}\left(\alpha_{2} b\right) K_{1}(\alpha, b)-\beta, b K_{0}(\alpha, b) K_{1}\left(\alpha_{2} b\right)\right]\right\}
$$

$\div\left\{\left[\beta_{2} b K_{0}\left(\alpha_{2} b\right) K_{1}(\alpha, b)-\beta, b K_{0}(\alpha, b) K_{1}\left(\alpha_{2} b\right)\right\}\right.$

$\left[\beta, a I_{0}(\alpha, a) I_{1}(\dot{a} a)-\alpha a I_{0}(\alpha a) I_{1}(\alpha, a)\right]+$

$\left[\alpha a I_{0}(\alpha a) K_{1}(\alpha, a)+\beta_{1} a K_{0}(\alpha, a) I_{1}(\alpha a)\right]$

$\left.\left[\beta, b I_{0}\left(\alpha_{1} b\right) K_{1}\left(\alpha_{2} b\right)+\beta_{2} b K_{0}\left(\alpha_{2} b\right) I_{1}(\alpha, b)\right]\right\}$

$\operatorname{Im}\left[\beta_{2} b K_{0}\left(\alpha_{2} b\right) K_{1}(\alpha, b)-\beta, b K_{0}(\alpha, b) K_{1}\left(\alpha_{2} b\right)\right]$

$\operatorname{Im}\left[\beta, a I_{0}(\alpha, a) I_{1}(\alpha a)-\alpha a I_{0}(\alpha a) I_{1}(\alpha, a)\right]$

$\operatorname{Im}\left[\alpha a I_{0}(\alpha a) K_{1}(\alpha, a)+\beta_{1} a K_{0}(\alpha, a) I_{1}(\alpha a)\right]$

$\operatorname{Im}\left[\beta, b I_{0}^{\prime}(\alpha, b) K_{1}\left(\alpha_{2} b\right)+\beta_{2} b K_{0}\left(\alpha_{2} b\right) I_{1}(\alpha, b)\right]$

$\operatorname{Im}\left\{K_{1}(\alpha, r)\left[\beta, b I_{0}(\alpha, b) K_{1}\left(\alpha_{2} b\right)+\beta_{2} b K_{0}\left(\alpha_{2} b\right) I_{1}(\alpha, b)\right]-\right.$

$$
\left.I_{1}\left(\alpha_{1} r\right)\left[\beta_{2} b K_{0}\left(\alpha_{2} b\right) K_{1}(\alpha, b)-\beta, b K_{0}\left(\alpha_{1} b\right) K_{1}\left(\alpha_{2} b\right)\right]\right\}
$$


DNR

DR1

DR2

DR3

DR4

S1

SNI

SNR

SSR

$\mathrm{X}$

$\mathrm{X} 1$

$X 1 A$

$\mathrm{X} 1 \mathrm{~B}$

$\mathrm{X} 1 \mathrm{R}$

$\mathrm{XA}$

$\mathrm{XB}$

$\mathrm{XF}$
$\operatorname{Re}\left\{K_{1}(\alpha, r)\left[\beta, b I_{0}(\alpha, b) K_{1}\left(\alpha_{2} b\right)+\beta_{2} b K_{0}\left(\alpha_{2} b\right) I_{1}(\alpha, b)\right]-\right.$

$$
\left.I_{1}(\alpha, r)\left\{\beta_{2} b K_{0}\left(\alpha_{2} b\right) K_{1}(\alpha, b)-\beta, b K_{0}(\alpha, b) K_{1}\left(\alpha_{2} b\right)\right\}\right\}
$$

$\operatorname{Re}\left[\beta_{2} b K_{0}\left(\alpha_{2} b\right) K_{1}\left(\alpha_{1} b\right)-\beta_{1} b K_{0}\left(\alpha_{1} b\right) K_{1}\left(\alpha_{2} b\right)\right]$

$\operatorname{Re}\left[\beta_{1} a I_{0}(\alpha, a) I_{1}(\alpha a)-\alpha a I_{0}(\alpha a) I_{1}(\alpha, a)\right]$

$\operatorname{Re}\left[\alpha a I_{0}(\alpha a) K_{1}(\alpha, a)+\beta_{1} a K_{0}(\alpha, a) I_{1}(\alpha a)\right]$

$\operatorname{Re}\left[\beta, b I_{0}(\alpha, b) K_{1}\left(\alpha_{2} b\right)+\beta_{2} b K_{0}\left(\alpha_{2} b\right) I_{1}(\alpha, b)\right]$

$d \alpha$

$$
\begin{array}{r}
\operatorname{Im}\left(-I_{1}\left(\alpha_{1} a\right)\left[\beta_{2} b K_{0}\left(\alpha_{2} b\right) K_{1}\left(\alpha_{1} b\right)-\beta_{1} b K_{0}\left(\alpha_{1} b\right) K_{1}\left(\alpha_{2} b\right)\right]+\right. \\
\left.K_{1}\left(\alpha_{1} a\right)\left[\beta, b I_{0}\left(\alpha_{1} b\right) K_{1}\left(\alpha_{2} b\right)+\beta_{2} b K_{0}\left(\alpha_{2} b\right) I_{1}(\alpha, b)\right]\right\} \\
\operatorname{Re}\left(-I_{1}\left(\alpha_{1} a\right)\left[\beta_{2} b K_{0}\left(\alpha_{2} b\right) K_{1}\left(\alpha_{1} b\right)-\beta_{1} b K_{0}\left(\alpha_{1} b\right) K_{1}\left(\alpha_{2} b\right)\right]+\right. \\
\left.K_{1}\left(\alpha_{1} a\right)\left[\beta_{1} b I_{0}\left(\alpha_{1} b\right) K_{1}\left(\alpha_{2} b\right)+\beta_{2} b K_{0}\left(\alpha_{2} b\right) I_{1}\left(\alpha_{1} b\right)\right]\right\}
\end{array}
$$

$$
\begin{aligned}
& \operatorname{Re} \frac{1}{I_{1}(\alpha a)}\left[-I_{1}(\alpha, a)\left[\beta_{2} b K_{0}\left(\alpha_{2} b\right) K_{1}(\alpha, b)-\beta_{1} b K_{0}(\alpha, b) K_{1}\left(\alpha_{2} b\right)\right]+\right. \\
& \left.K_{1}(\alpha, a)\left[\beta, b I_{0}(\alpha, b) K_{1}\left(\alpha_{2} b\right)+\beta_{2} b K_{0}\left(\alpha_{2} b\right) I_{1}(\alpha, b)\right]\right] \\
& {\left[\left[\beta_{2} b K_{0}\left(\alpha_{2} b\right) K_{1}(\alpha, b)-\beta_{1} b K_{0}(\alpha, b) K_{1}\left(\alpha_{2} b\right)\right]\right.} \\
& {\left[\beta_{1} a I_{0}(\alpha, a) I_{1}(\alpha a)-\alpha a I_{0}(\alpha a) I_{1}(\alpha, a)\right]} \\
& {\left[\alpha a I_{0}(\alpha a) K_{1}(\alpha, a)+\beta_{1} a K_{0}(\alpha, a) I_{1}(\alpha a)\right]} \\
& \left.\left[\beta, b I_{0}(\alpha, b) K_{1}(\alpha, b)+\beta_{2} b K_{0}(\alpha, b) I_{1}(\alpha, b)\right]\right]^{-1}-\frac{K_{1}(\alpha a)}{I_{1}(\alpha a)}
\end{aligned}
$$

$\alpha$

$\operatorname{Re}\left[\alpha_{1}\right]$

$\operatorname{Re}[\alpha, a]$

$$
\operatorname{Re}[\alpha, b]
$$

$\operatorname{Re}[\alpha, r]$

$\alpha a$

$\alpha b$

$\frac{4}{\pi} \frac{1}{\alpha^{3}} I\left(r_{2}, r_{1}\right) \sin (\alpha \ell / 2) d \alpha$ 
$\mathrm{XF1}$

$\frac{4}{\pi} \frac{1}{\alpha^{3}} I\left(r_{2}, r_{1}\right) \sin (\alpha \ell / 2) \sin \left(\frac{\alpha c}{2}\right) d \alpha$

XF2

$\frac{4}{\pi} \frac{1}{\alpha^{3}} I\left(r_{2}, r_{1}\right) \sin (\alpha \ell / 2) \cos \left(\frac{\alpha c}{2}\right) d \alpha$

XFACT

$\frac{1}{\alpha^{3}} I\left(r_{2}, r_{1}\right) \sin (\alpha \ell / 2)$

XFACT1

$\frac{4}{\pi} \frac{1}{\alpha^{3}} I\left(r_{2}, r_{1}\right) \sin (\alpha \ell / 2) \sin \left(\frac{\alpha c}{2}\right) \sin (\alpha z) d \alpha$

XFACT2

$\frac{4}{\pi} \frac{1}{\alpha^{3}} I\left(r_{2}, r_{1}\right) \sin (\alpha \ell / 2) \cos \left(\frac{\alpha c}{2}\right) \cos (\alpha z) d \alpha$

XIR21

$\frac{1}{\alpha^{3}} I\left(r_{2}, r_{1}\right)$

$\mathrm{XJR} 21$

$\frac{1}{\alpha^{3}} J\left(r_{2}, r_{1}\right)$

$\mathrm{XL}$

$\alpha \ell$

$\mathrm{XX}$

$\alpha^{2}$

$\mathrm{xxxx}$

$\alpha^{4}$

Y1

$\operatorname{Im}\left[\alpha_{1}\right]$

Y1A

$\operatorname{Im}[\alpha, a]$

Y1B

$\operatorname{Im}[\alpha, b]$

Y1R

$\operatorname{Im}[\alpha, r]$

ZIOIA

$\operatorname{Im}\left[\alpha, a I_{0}(\alpha, a)\right]$

ZIOIB

$\operatorname{Im}\left[\alpha, b I_{0}(\alpha, b)\right]$

ZIOTR

$\operatorname{Tm}\left[\alpha_{1} r T_{0}\left(\alpha_{1} r\right)\right]$

ZIORA

$\operatorname{Re}\left[\alpha, a I_{0}(\alpha, a)\right]$

ZIORB

$\operatorname{Re}\left[\alpha, b I_{0}(\alpha, b)\right]$

ZIORR

$\operatorname{Re}\left[\alpha, r I_{0}(\alpha, r)\right]$

ZKOIA

$\operatorname{Im}\left[\alpha, a K_{0}(\alpha, a)\right]$

ZKOIB

$\operatorname{Im}\left[\alpha, b K_{0}(\alpha, b)\right]$

ZKOIR.

$\operatorname{Tm}\left[\alpha_{1} r K_{0}(\alpha, r)\right]$

ZKORA

$\operatorname{Re}\left[\alpha, a K_{0}(\alpha, a)\right]$

ZKORB

$\operatorname{Re}\left[\alpha_{1} b K_{0}(\alpha, b)\right]$

ZKORR

$\operatorname{Re}\left[\alpha, r K_{0}(\alpha, r)\right]$ 


\section{Sample output}

The program DFBORAR calculates the defect signal averaged over the depth of the defect at different distances along the tube. The program can plot the defect impedance as the tube is scanned and pick out the maximum magnitude. Below we show the printer output of a sample run where the maximum signal is printed.

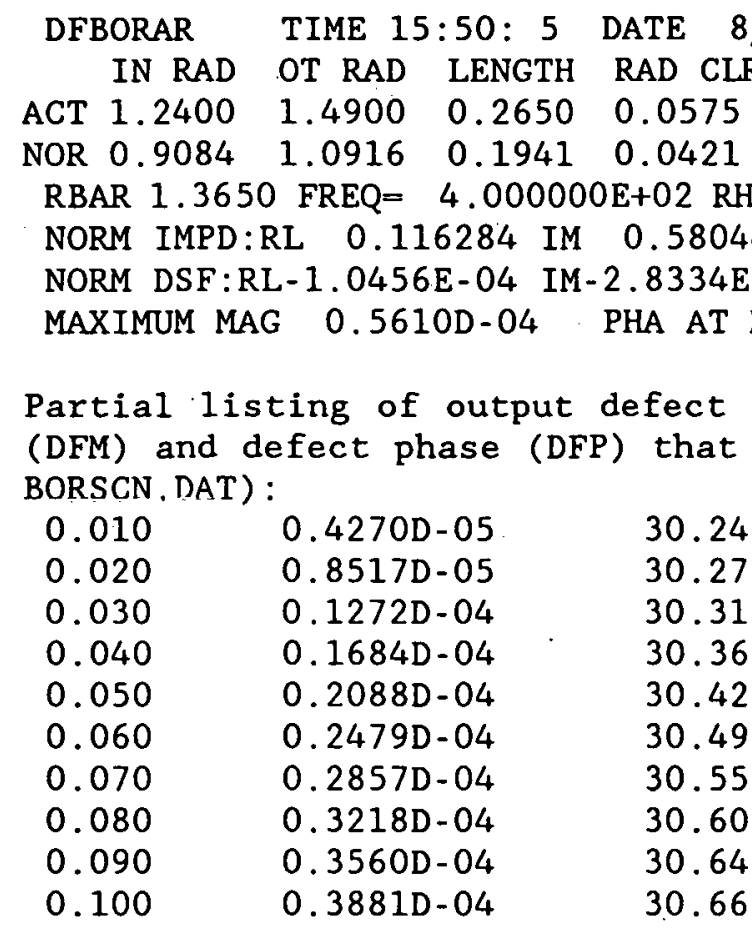

If the proper plotting software has been installed, a plot of this data is made on the CRT and a hard copy can be obtained, as shown in Fig. 17. The plot forms one-half the normal Lissajous pattern one gets in an eddycurrent test with a differential bobbin coil. The second half can be obtained by reflecting the signal in the $-z$ direction, since the signal is anti-symmetric. The phases and magnitudes given in this report are referenced to the $X$ axis being zero phase and measured counterclockwise from $X$ axis, which is standard for mathematics and electrical engineering. Standard eddy-current practice is to measure the phase shift in a clockwise direction from the $-X$ axis. Furthermore, the phase is rotated until the probe wobble/fill factor/lift-off variations lie in a horizontal direction. 
ORNL-DWG $90-8097$

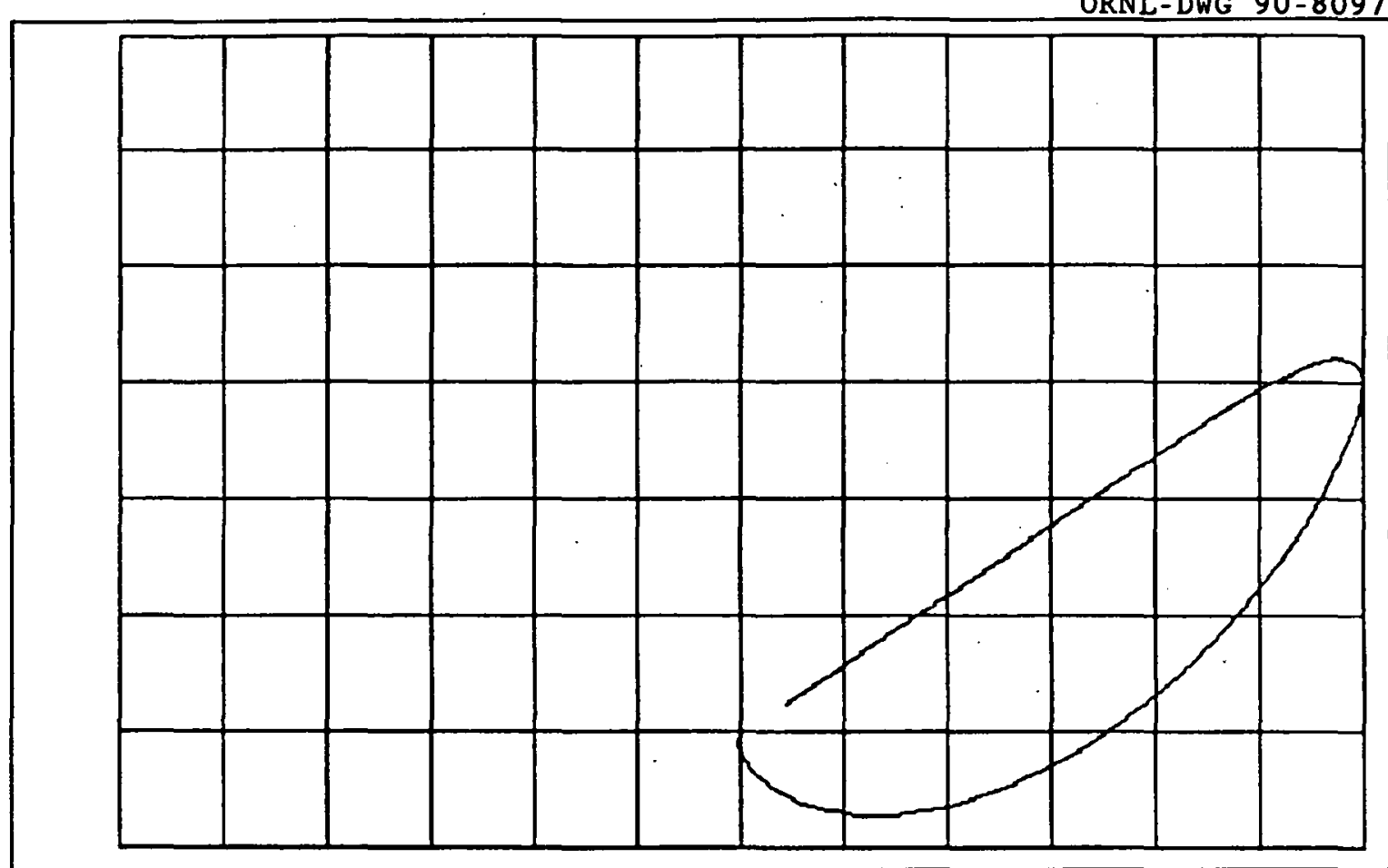

Fig. 17. Plot of defect signal on complex impedance plane as the defect is moved from the probe center in the plus $z$ direction. 


\section{Listing}

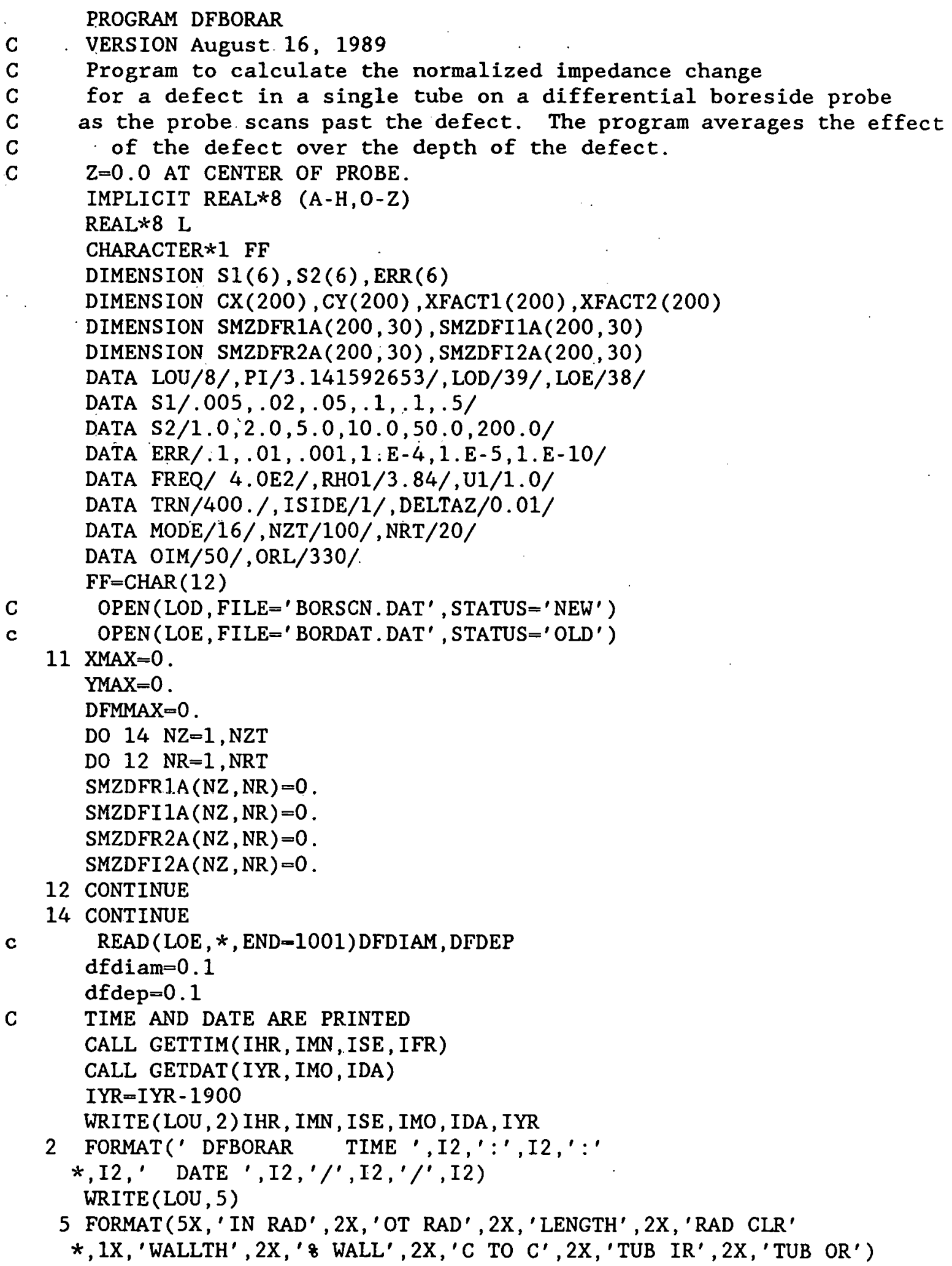




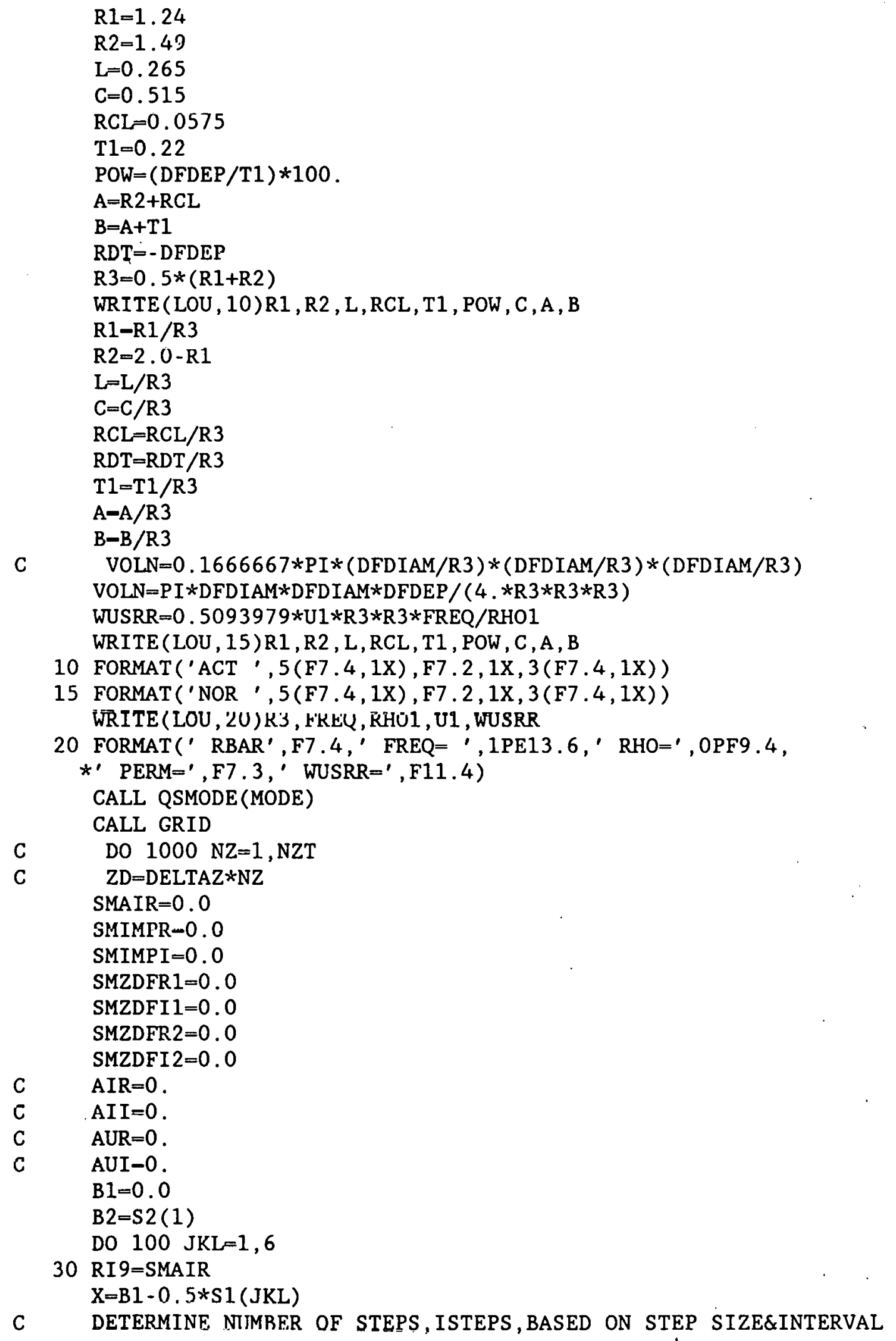


ISTEPS $=\operatorname{DNINT}((\mathrm{B} 2-\mathrm{B} 1) / \mathrm{S} 1(\mathrm{JKL}))$

DO $90 \mathrm{I}=1$, ISTEPS

$\mathrm{X}=\mathrm{X}+\mathrm{S} 1(\mathrm{JKL})$

CALL IJBSSL(X,R1,R2,XIR21, XJR21)

$\mathrm{XL}=\mathrm{X} * \mathrm{~L}$

IF (XI.GT.5.OE-3) GO TO 60

$\mathrm{A} 1=\mathrm{XL} * \mathrm{XL} *(0.5-\mathrm{XL} / 6.0)$

GO TO 80

60 IF(XL.GT.75.0) GO TO 70

$\mathrm{Al}=\mathrm{XL}+\mathrm{DEXP}(-\mathrm{XL})-1.0$

GO TO 80

$70 \mathrm{~A} 1=\mathrm{XL}-1.0$

80 SMAIR $=$ SMAIR $+2 . * \mathrm{XJR} 21 * \mathrm{XJR} 21 * \mathrm{~A} 1 * \mathrm{~S} 1(\mathrm{JKL})$

IF (X.GT . 160.) GO TO 90

$\mathrm{XX}=\mathrm{X} * \mathrm{X}$

$\mathrm{XXXX}=\mathrm{XX} * \mathrm{XX}$

$\mathrm{X} 1=\mathrm{DSQRT}(0.5 *(\mathrm{XX}+\mathrm{DSQRT}(\mathrm{XXXX}+\mathrm{WUSRR} * \mathrm{WUSRR}))) / \mathrm{U} 1$

$\mathrm{Y} 1=\mathrm{WUSRR} /(2 * \mathrm{X} 1 * \mathrm{U} 1 * \mathrm{U} 1)$

$X A=X * A$

$\mathrm{XB}=\mathrm{X} * \mathrm{~B}$

$\mathrm{X} 1 \mathrm{~A}=\mathrm{X} 1 * \mathrm{~A}$

$\mathrm{Y} 1 \mathrm{~A}=\mathrm{Y} 1 * \mathrm{~A}$

$X 1 B=X 1 * B$

$\mathrm{Y} 1 \mathrm{~B}=\mathrm{Y} 1 * \mathrm{~B}$

CALL CMDBES (X1A, Y1A, ZIORA, ZIOIA, ZKORA, ZKOIA, BI1RA, BIIIA

*, BK1RA, BK1IA)

CALL CMDBES (X1B , Y1B , ZIORB , ZIOIB , ZKORB , ZKOIB , BI1RB , BIIIB

*, BK1RB, BK1IB)

CALL BESI (XA, BIOA, BIIA)

CALL BESK(XA,BKOA,BKIA)

CALL BESI (XB, BIOB, BI1B)

CALL BESK (XB, BKOB, BK1B)

$\mathrm{DR} 1=\mathrm{XB} * \mathrm{BKOB} * \mathrm{BK} 1 \mathrm{RB}-\mathrm{ZKORB} * \mathrm{BK} 1 \mathrm{~B} / \mathrm{U} 1$

$\mathrm{DI} 1=\mathrm{XB} * \mathrm{BKOB} * \mathrm{BK} 1 \mathrm{IB}-\mathrm{ZKOIB} * \mathrm{BK} 1 \mathrm{~B} / \mathrm{U} 1$

$D R 2=Z I O R A * B I 1 A / U 1-X A * B I O A * B I 1 R A$

DI2 $=2$ IOIA $* B I 1 A / U 1-X A * B I O A * B I 1 I A$

$\mathrm{DR} 3=\mathrm{XA} * \mathrm{BIOA} * \mathrm{BK} 1 \mathrm{RA}+\mathrm{ZKORA} * \mathrm{BI} 1 \mathrm{~A} / \mathrm{U} 1$

$\mathrm{DI} 3=\mathrm{XA} * \mathrm{BIOA} * \mathrm{BK} 1 \mathrm{IA}+\mathrm{ZKOIA} * \mathrm{BI} 1 \mathrm{~A} / \mathrm{U} 1$

$\mathrm{DR} 4=\mathrm{ZIORB} * \mathrm{BK} 1 \mathrm{~B} / \mathrm{U} 1+\mathrm{XB} * \mathrm{BKOB} * \mathrm{BIIRB}$

$\mathrm{DI} 4=\mathrm{ZIOIB} * \mathrm{BK} 1 \mathrm{~B} / \mathrm{U} 1+\mathrm{XB} * \mathrm{BKOB} * \mathrm{BI} 1 \mathrm{IB}$

$\mathrm{DDR}=\mathrm{DR} 1 * \mathrm{DR} 2-\mathrm{DI} 1 * \mathrm{DI} 2+\mathrm{DR} 3 * \mathrm{DR} 4-\mathrm{DI} 3 * \mathrm{DI} 4$

$\mathrm{DDI}=\mathrm{DR} 1 * \mathrm{DI} 2+\mathrm{DR} 2 * \mathrm{DI} 1+\mathrm{DR} 3 * \mathrm{DI} 4+\mathrm{DR} 4 * \mathrm{DI} 3$

$S N R=-B I 1 R A * D R 1+B I 1 I A * D I 1+B K 1 R A * D R 4-B K 1 I A * D I 4$

$S N I=-B I 1 I A * D R I-B I 1 R A * D I 1+B K 1 R A * D I 4+B K 1 I A * D R 4$

$\mathrm{DEN}=\mathrm{DDR} * \mathrm{DDR}+\mathrm{DDI} * \mathrm{DDI}$

$\mathrm{SSR}=((\mathrm{SNR} * \mathrm{DDR}+\mathrm{SNI} * \mathrm{DDI}) / \mathrm{DEN}-\mathrm{BK} 1 \mathrm{~A}) / \mathrm{BI} 1 \mathrm{~A}$

$S S I=(S N I * D D R-S N R * D D I) /(B I 1 A * D E N)$

$\mathrm{XFACT}=\mathrm{XIR} 21 * \mathrm{DSIN}(\mathrm{XL} / 2$.

$\mathrm{SMIMPR}=\mathrm{SMIMPR}+8 . * \mathrm{XFACT} * \mathrm{XFACT} * \mathrm{~S} 1(\mathrm{JKL}) * \mathrm{SSR} / \mathrm{PI}$

SMIMPI $=S M I M P I+8 . * X F A C T * X F A C T * S 1(J K L) * S S I / P I$

$\mathrm{XF}=4 . * \mathrm{XFACT} * \mathrm{~S} 1(\mathrm{JKL}) / \mathrm{PI}$ 
$\mathrm{XF} 1=\mathrm{XF} * \mathrm{DS} \operatorname{IN}(0.5 * \mathrm{X} * \mathrm{C})$

$\mathrm{XF} 2=\mathrm{XF} * \mathrm{DCOS}(0.5 * \mathrm{X} * \mathrm{C})$

DO $89 \mathrm{NR}=1$, NRT

$\mathrm{RD}=(\operatorname{REAL}(\mathrm{NR})-0.5) *(\mathrm{RDT} / \mathrm{REAL}(\mathrm{NRT}))$

IF (ISIDE.EQ.1) THEN

$\mathrm{RD}=\mathrm{B}+\mathrm{RD}$

ELSE

$\mathrm{RD}=\mathrm{A}-\mathrm{RD}$

END IF

$\mathrm{X} 1 \mathrm{R}=\mathrm{X} 1 * \mathrm{RD}$

$\mathrm{Y} 1 \mathrm{R}-\mathrm{Y} I * \mathrm{RD}$

CALL CMDBES (X1R, Y1R, ZIORR, ZIOIR, ZKORR, ZKOIR, BIIRR, BI1IR.

*, BK1RR, BK1IR)

$\mathrm{DNR}-\mathrm{BK} 1 \mathrm{RR} * \mathrm{DR} 4-\mathrm{BK} 1 \mathrm{IR} * \mathrm{DI} 4-\mathrm{BI} 1 \mathrm{RR} * \mathrm{DR} 1+\mathrm{BI} 1 \mathrm{IR} * \mathrm{DI} 1$

$D N I=B K 1 I R * D R 4+B K 1 R R * D I 4-B I 1 I R * D R 1-B I 1 R R * D I 1$

$\mathrm{DFR}=(\mathrm{DNR} * \mathrm{DDR}+\mathrm{DNI} * \mathrm{DDI}) / \mathrm{DEN}$

$\mathrm{DFI}=(\mathrm{DNI} * \mathrm{DDR}-\mathrm{DNR} * \mathrm{DDI}) / \mathrm{DEN}$

Do $88 \mathrm{NZ}=1$, NZT

IF (NR.GT.1)GO TO 87

$\mathrm{ZD}=\mathrm{DELTA} * \mathrm{NZ}$

$\mathrm{XFACT} 1(\mathrm{NZ})=\mathrm{XF} 1 * \mathrm{DSIN}(\mathrm{X} * \mathrm{ZD})$

$\mathrm{XFACT} 2(\mathrm{NZ})=\mathrm{XF} 2 * \mathrm{DCOS}(\mathrm{X} * \mathrm{ZD})$

87 SMZDFR $1 A(N Z, N R)=\operatorname{SMZDFR} 1 A(N Z, N R)+X F A C T 1(N Z) * D F R$

$\operatorname{SMZDFI} 1 A(N Z, N R)=\operatorname{SMZDFI} 1 A(N Z, N R)+\operatorname{XFACT1}(\mathrm{NZ}) * \mathrm{DFI}$

$\operatorname{SMZDFR} 2 A(N Z, N R)=\operatorname{SMZDFR} 2 A(N Z, N R)+\operatorname{XFACT} 2(\mathrm{NZ}) * \mathrm{DFR}$

SMZDFI 2A (NZ, NR) $=$ SMZDFI $2 A(N Z, N R)+X F A C T 2(N Z) * D F I$

88 CONTINUE

89 CONTINUE

90 CONTINUE

$\mathrm{B} 1=\mathrm{B} 2$

$\mathrm{B} 2=\mathrm{B} 2+\mathrm{S} 2(\mathrm{JKL})$

CHECK $=($ SMAIR - RI9) /SMAIR

IF (ABS (CHECK) .GT.ERR(JKL)) GO TO 30

100 CONTINUE

DO $990 \mathrm{NZ}=1, \mathrm{NZT}$

$\mathrm{ZD}=\mathrm{DELTAZ} * \mathrm{NZ}$

SMZDFR $1=0$.

SMZDFI $1=0$.

SMZDFR2 $=0$.

SMZDFI $2=0$.

DO $120 \mathrm{NR}=1$, NRT

SMZDFR 1 =SMZDFR1+SMZDFR1A (NZ, NR)

SMZDFI1-SMZDFI1 I SMZDFI IA (NZ, NR)

SMZDFR2=SMZDFR2+SMZDFR2A(NZ,NR)

SMZDFI2=SMZDFI2+SMZDFI2A (NZ,NR)

120 CONTINUE

SMZDFR $1=S M Z D F R 1 /$ NRT

SMZDFI $1=$ SMZDFI1/NRT 


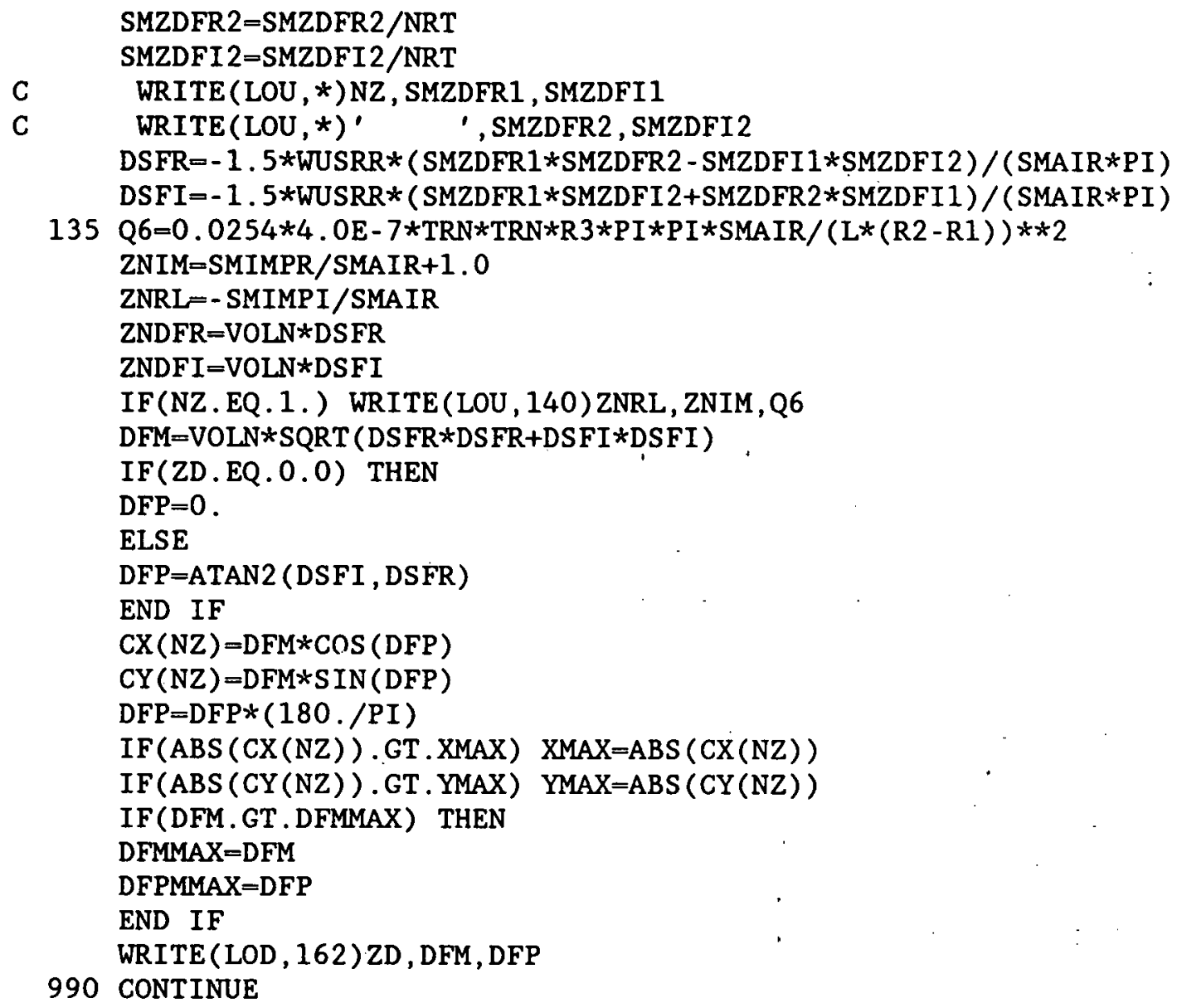


CALL QLINE(IR1, IM1, IR2, IM2, 15)

IR1 $=$ IR2

IM1=IM2

1000 CONTINUE

WRITE (LOU, *)

WRITE (LOU, *)

CALL PRTSC

WRITE (LOU, *)FF

c GO TO 11 .

140 FORMAT(' NORM IMPD:RL', F10.6,". IM" ,F10;.6, *' AIR IND',1PE13.6)

C 150 FORMAT(' NORM CHG:RL ',OPF10.6," IM" ,OPF10.6,

C *'MAG',OPF10.6,' PHA ',OPFT.2', ();

160 FORMAT(' NORM DSF:RL', 1PE11 .4,' IM!", IPEI1..4,' VOLN', 1PE11.4)

161 FORMAT(' ZD MAG

162 FORMAT (F6.3, 5X, D11.4, 5X, F7.2)

164 FORMAT(' MAXIMUM MAG ',D11.4,' PHA AT MAX MAG ',F7.2, V)

1001 END 
The programs in this section perform functions relating to the effect on an encircling coil of a defect in a single conducting tube. The types of circumferential coils dealt with in this section are absolute encircling and differential encircling coils. Calculations of the normalized impedance change in these coils due to a defect in a single conducting tube are done by programs ABENCAR and DFENCAR. Fig: 18 shows a differential coil encircling a tube.

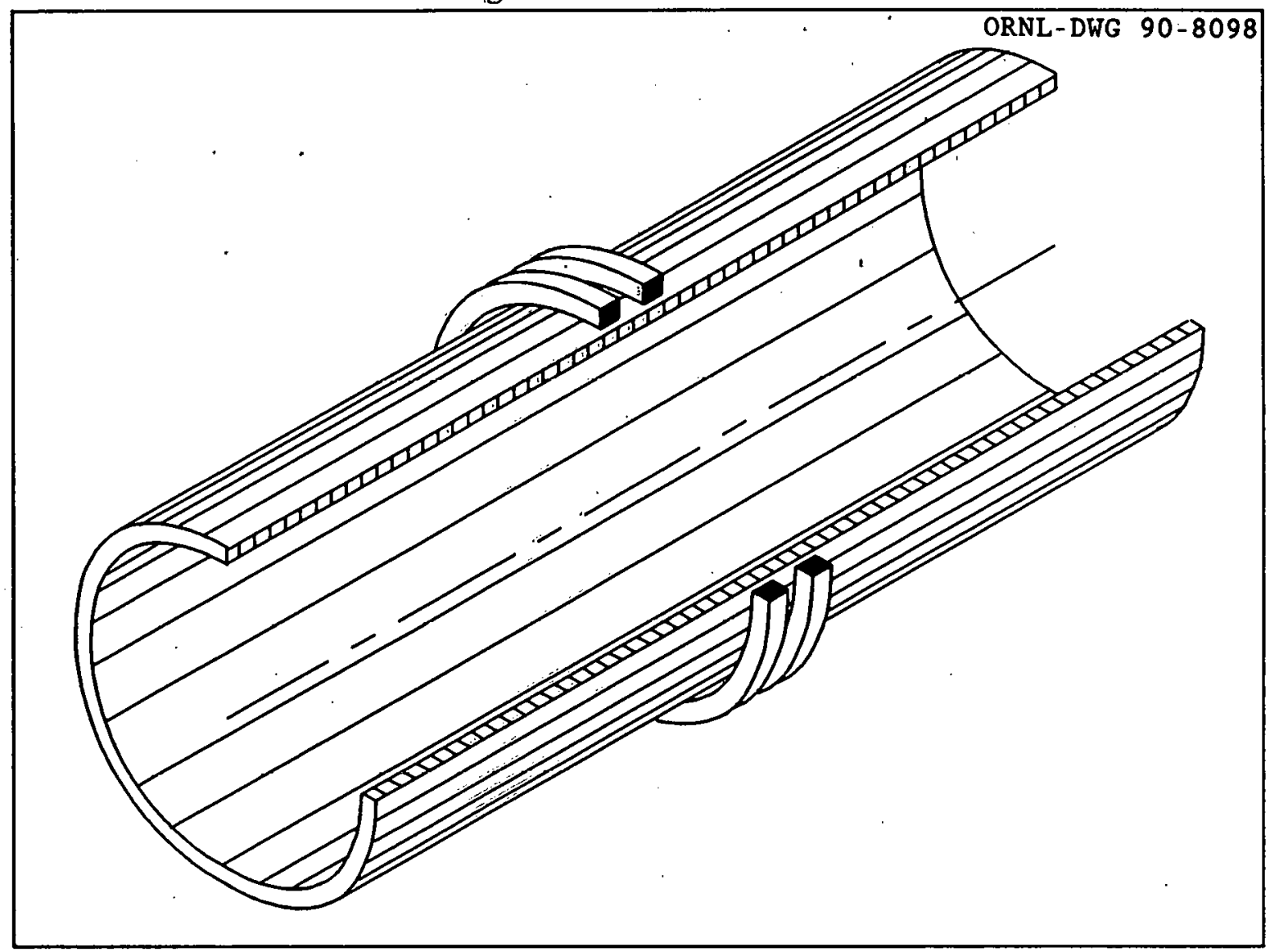

Fig. 18. Differential coil encirclïng a conducting tube. 
ABENCAR calculates impedance change for absolute coil

Program ABENCAR calculates the change in the normalized impedance of an absolute encircling coil due to the presence of a defect in a single conducting tube, as shown in Fig. 19.

ORNL-DWG $90-8100$

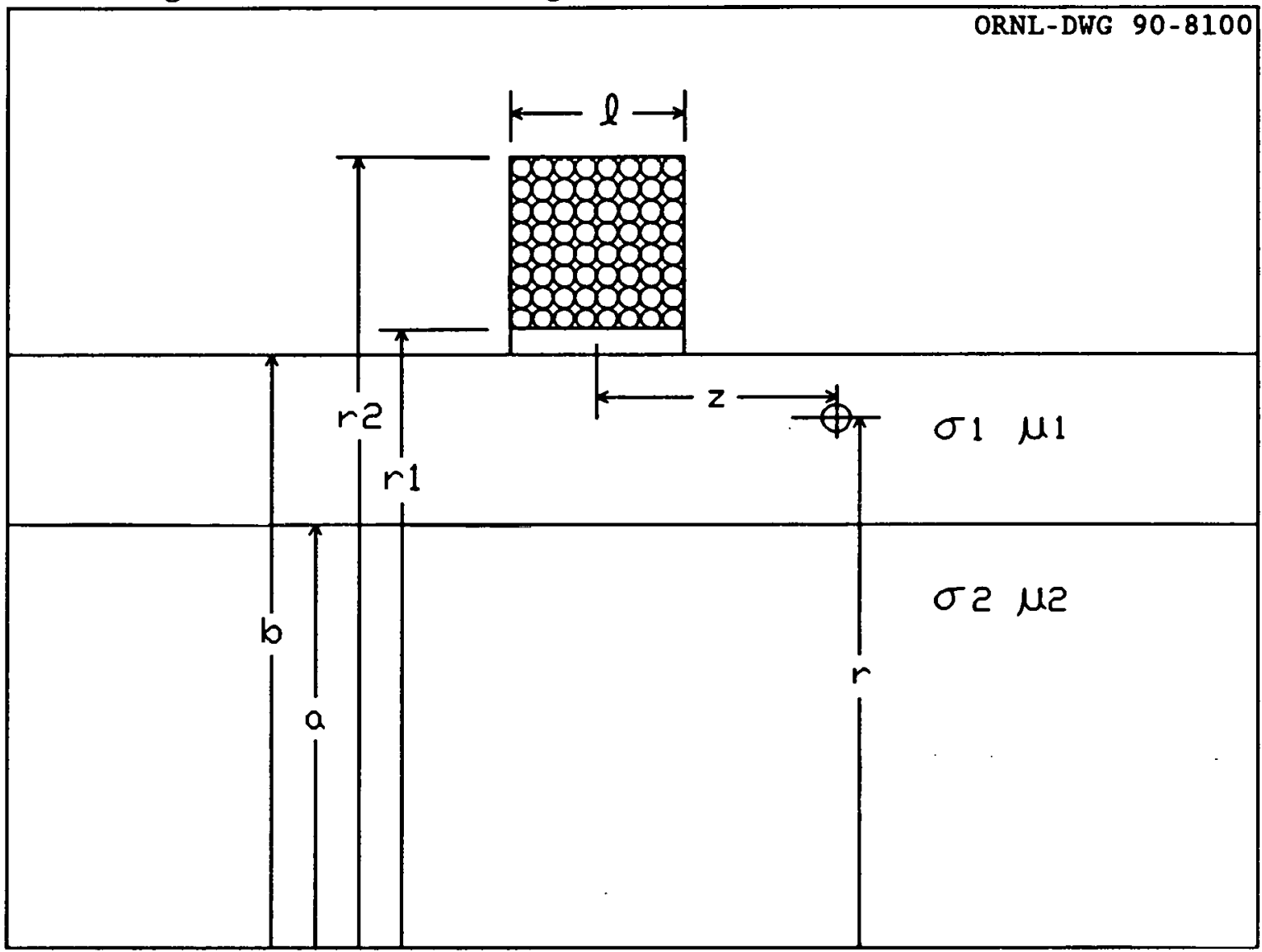

Fig. 19. Absolute coil encircling a tube in the presence of a defect.

The normalized impedance for a coil encircling a tube, without the defect, is :

$Z_{\mathrm{n}}=\frac{j}{I_{\mathrm{arr}}} \int_{0}^{\infty}\left[\frac{8}{\pi \alpha^{6}} K^{2}\left(r_{2}, r_{1}\right)\left\{\frac{K_{1}(\alpha, b) D_{2}+I_{1}(\alpha, b) D_{3}}{K_{1}(\alpha b)\left(D_{1} D_{2}+D_{3} D_{4}\right)}-\frac{I_{1}(\alpha b)}{K_{1}(\alpha b)}\right\} \sin ^{2}\left(\frac{\alpha \ell}{2}\right)+I_{\mathrm{arr}}\right] d \alpha$

and for the change in the normalized impedance due to the defect we have:

$Z_{n \sigma}(r, z)=\frac{-3\left(\omega \mu \sigma, \bar{r}^{2}\right) \operatorname{Vol} I_{n}}{2 \pi I_{\mathrm{a} \mid r}}\left[\int_{0}^{m} \frac{K\left(r_{2}, r_{1}\right)}{\pi \alpha^{3}}\left\{\frac{K_{1}(\alpha, r) D_{2}+I_{1}\left(\alpha_{1} r\right) D_{3}}{\left(D_{1} D_{2}+D_{3} D_{4}\right)}\right\} \sin \left(\frac{\alpha \ell}{2}\right) 2 \cos (\alpha z) d \alpha\right]^{2}$

where: 


$$
\begin{aligned}
& D_{1}=\alpha b K_{0}(\alpha b) K_{1}\left(\alpha_{1} b\right)-\beta_{1} b K_{0}\left(\alpha_{1} b\right) K_{1}(\alpha b) \\
& D_{2}=\beta_{1} a I_{0}(\alpha, a) I_{1}\left(\alpha_{2} a\right)-\beta_{2} a I_{0}\left(\alpha_{2} a\right) I_{1}\left(\alpha_{1} a\right) \\
& \vdots=\beta_{2} a I_{0}\left(\alpha_{2} a\right) K_{1}\left(\alpha_{1} a\right)+\beta_{1} a K_{0}\left(\alpha_{1} a\right) I_{1}\left(\alpha_{2} a\right) \\
& D_{4}=\beta_{1} b I_{0}\left(\alpha_{1} b\right) K_{1}(\alpha b)+\alpha b K_{0}(\alpha b) I_{1}\left(\alpha_{1} b\right) \\
& K\left(r_{2}, r_{1}\right)=\int_{\alpha r_{1}}^{a r_{2}} x K_{1}(x) d x \\
& \alpha_{1}=\left(\alpha^{2}+j \omega \mu \sigma_{1} \bar{r}^{2}\right)^{1 / 2}
\end{aligned}
$$

The term $I_{\text {ail. }}$ is related to the air inductance of the coil and is:

$$
I_{\mathrm{air}}=\int_{0}^{\infty} \frac{1}{\alpha^{6}}\left[J\left(r_{2}, r_{1}\right)\right]^{2} 2[\alpha \ell+\exp (-\alpha \ell)-1] d \alpha
$$

where $J\left(r_{2}, r_{1}\right)=\int_{\alpha r_{1}}^{\alpha r_{2}} x J,(x) d x$

The program performs the calculations for a number of different axial distances between the coil and the defect, and it can divide the defect into a number of parts and calculate the effect of each part separately. In the inner conductor, the value of the conductivity $\sigma_{2}$ is taken as zero, and the relative permeability $\mu_{2}$ is taken as unity, so that $\beta_{2}$ becomes $\alpha$. These values are used in the program, but not in the derivation.

\section{Variables}

A number of the variables which occur mainly inside the integration loops do not correspond to anything physical. These variables are described in a section at the end. Starred variables must be assigned by the user.
A
B
The normalized inner radius of the tube.
DELTAZ
The normalized outer radius of the tube.
The normalized axial distance between the points at which the calculations are done.
DFDEP' The depth of the defect in the tube in inches.
DFDIAM The diameter of the defect in the tube in inches.
DFM
The magnitude of the normalized impedance change in the coil due to the defect.
DFP
The phase of the normalized impedance change in the coil due to the defect. 


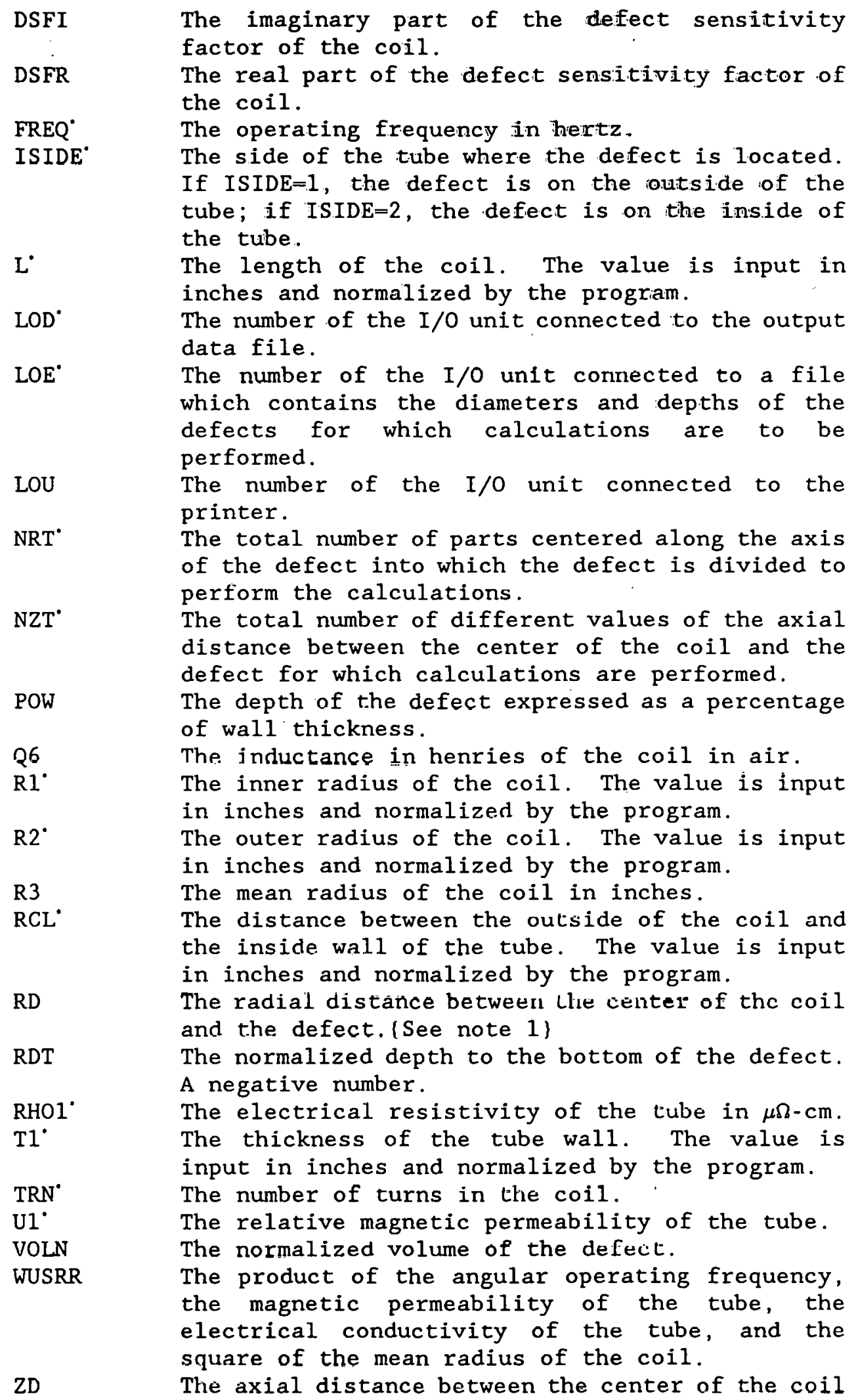


and the defect.

ZNDFI The imaginary part of the normalized impedance change in the coil due to the defect.

ZNDFR The real part of the normalized impedance change in the coil due to the defect.

ZNIM The imaginary part of the normalized impedance of the coil when no defects are present.

ZNRL The real part of the normalized impedance of the coil when no defects are present.

Integration Section of Program ABENGAR

\section{Symbol definitions}

The following are definitions of the symbols used to describe the program variables which appear in the integration section of the program. All lengths are normalized unless otherwise noted.

$\alpha$
$a$
$\beta_{1}$
$b$
$I_{0}(x)$
$I_{1}(x)$
$J\left(x_{2}, x_{1}\right)$
$J_{1}(x)$
$K\left(x_{2}, x_{1}\right)$
$K_{0}(x)$
$K_{1}(x)$
$\ell$
$\mu$
$\sigma_{1}$
$r$
$r$
$r_{1}$
$r_{2}$
$z$
$\omega$

\section{Integration variable}

Inner radius of the tube $\left(\alpha^{2}+j \omega \mu \sigma, \bar{r}^{2}\right)^{1 / 2} / \mu$

Outer radius of the tube

Modified Bessel function of the first kind of order 0

Modified Bessel function of the first kind of order 1

Integral of $x J_{1}(x)$ with respect to $x$ from $\alpha x_{1}$ to $\alpha x_{2}$

Bessel function of the first kind of order 1

Integral of $x K_{1}(x)$ with respect to $x$ from $\alpha x_{1}$ to $\alpha x_{2}$

Modified Bessel function of the second kind of order 0

Modified Bessel function of the second kind of order 1

Length of the coil

Relative magnetic permeability of the tube

Electrical conductivity of the tube

Radial distance between center of coil and defect

Coil mean radius in inches

Inner radius of coil

Outer radius of coil

Axial distance between defect and coil center

Angular operating frequency

\section{Variables appearing in the integration section}

\begin{tabular}{|c|c|}
\hline $\begin{array}{l}\text { Program } \\
\text { variable }\end{array}$ & $\begin{array}{l}\text { Symbolic } \\
\text { equivalent }\end{array}$ \\
\hline B:IOA & $I_{0}(\alpha a)$ \\
\hline BIOB & $I_{0}(\alpha b)$ \\
\hline BIIA & $I_{1}(\alpha a)$ \\
\hline BIIB & $I_{1}(\alpha b)$ \\
\hline
\end{tabular}




\begin{tabular}{|c|c|}
\hline BI1IA & $\operatorname{Im}\left[I_{1}(\alpha, a)\right]$ \\
\hline BI1IB & $\operatorname{Im}\left[I_{1}(\alpha, b)\right]$ \\
\hline BI1IR & $\operatorname{Im}\left[I_{1}\left(\alpha_{1} r\right)\right]$ \\
\hline BI1RA & $\operatorname{Re}\left[I_{1}(\alpha, a)\right]$ \\
\hline BI1RB & $\operatorname{Re}\left[I_{1}(a, b)\right]$ \\
\hline BI1RR & $\operatorname{Re}\left[I_{1}(\alpha, r)\right]$ \\
\hline BKOA & $K_{0}(\alpha a)$ \\
\hline BKOB & $K_{0}(\alpha b)$ \\
\hline BKlA & $K_{1}(\alpha a)$ \\
\hline BK1B & $K_{1}(a b)$ \\
\hline BK1IA & $\operatorname{Im}\left[K_{1}(\alpha, a)\right]$ \\
\hline BK1IB & $\operatorname{Im}\left[K_{1}(\alpha, b)\right]$ \\
\hline BK1IR & $\operatorname{Im}\left[K_{1}(\alpha, r)\right]$ \\
\hline BK1RA & $\operatorname{Re}\left[K_{1}(\alpha, a)\right]$ \\
\hline BK1RB & $\operatorname{Re}\left[K_{1}(\alpha, b)\right]$ \\
\hline BK1RR & $\operatorname{Re}\left[K_{1}(\alpha, r)\right]$ \\
\hline \multirow[t]{4}{*}{ DDI } & $\operatorname{Im}\left(\left[\alpha b K_{0}(\alpha b) K_{1}(\alpha, b)-\beta_{1} b K_{0}(\alpha, b) K_{1}(\alpha b)\right]\right.$ \\
\hline & {$\left[\beta, a I_{0}(\alpha, a) I_{1}\left(\alpha_{2} a\right)-\beta_{2} a I_{0}\left(\alpha_{2} a\right) I_{1}(\alpha, a)\right]+$} \\
\hline & {$\left[\beta_{2} a I_{0}\left(\alpha_{2} a\right) K_{1}\left(\alpha_{1} a\right)+\beta_{1} a K_{0}\left(\alpha_{1} a\right) I_{1}\left(\alpha_{2} a\right)\right]$} \\
\hline & $\left.\left[\beta, b I_{0}(\alpha, b) K_{1}(\alpha b)+\alpha b K_{0}(\alpha b) I_{1}(\alpha, b)\right]\right\}$ \\
\hline \multirow[t]{4}{*}{$\mathrm{DDR}$} & $\operatorname{Re}\left(\left[\alpha b K_{0}(\alpha b) K_{1}(\alpha, b)-\beta_{1} b K_{0}(\alpha, b) K_{1}(\alpha b)\right]\right.$ \\
\hline & {$\left[\beta_{1} a I_{0}(\alpha, a) I_{1}\left(\alpha_{2} a\right)-\beta_{2} a I_{0}\left(\alpha_{2} a\right) I_{1}(\alpha, a)\right]+$} \\
\hline & {$\left[\beta_{2} a I_{0}\left(\alpha_{2} a\right) K_{1}(\alpha, a)+\beta_{1} a K_{0}(\alpha, a) I_{1}\left(\alpha_{2} a\right)\right]$} \\
\hline & $\left.\left[\beta, b I_{0}(\alpha, b) K_{1}(\alpha b)+\alpha b K_{0}(\alpha b) I_{1}(\alpha, b)\right]\right\}$ \\
\hline \multirow[t]{6}{*}{ DFR } & $\operatorname{Re}\left\{K_{1}(\alpha, r)\left[\beta_{1} a I_{0}(\alpha, a) I_{1}\left(\alpha_{2} a\right)-\beta_{2} a I_{0}\left(\alpha_{2} a\right) I_{1}(\alpha, a)\right]+\right.$ \\
\hline & $\left.I_{1}(\alpha, r)\left[\beta_{2} a I_{0}\left(\alpha_{2} a\right) K_{1}(\alpha, a)+\beta_{1} a K_{0}(\alpha, a) I_{1}\left(\alpha_{2} a\right)\right]\right\}$ \\
\hline & $\div\left\{\left[\alpha b K_{0}(\alpha b) K_{1}(\alpha, b)-\beta, b K_{0}(\alpha, b) K_{1}(\alpha b)\right]\right.$ \\
\hline & {$\left[\beta, a I_{0}\left(\alpha_{1} a\right) I_{1}\left(\alpha_{2} a\right)-\beta_{2} a I_{0}\left(\alpha_{2} a\right) I_{1}\left(\alpha_{1} a\right)\right]+$} \\
\hline & {$\left[\beta_{2} a I_{0}\left(\alpha_{2} a\right) K_{1}\left(\alpha_{1} a\right)+\beta_{1} a K_{0}\left(\alpha_{1} a\right) I_{1}\left(\alpha_{2} a\right)\right]$} \\
\hline & $\left.\left[\beta, b I_{0}(\alpha, b) K_{1}(\alpha b)+\alpha b K_{0}(\alpha b) I_{1}(\alpha, b)\right]\right\}$ \\
\hline DI1 & $\operatorname{Im}\left[\alpha b K_{0}(\alpha b) K_{1}(\alpha, b)-\beta, b K_{0}(\alpha, b) K_{1}(\alpha b)\right]$ \\
\hline
\end{tabular}


DI2

DI3

DI4

DNI

DNR

DR1

DR2

DR3

DR4

S1

SNI

SNR

SSR

$\mathrm{X}$

$\mathrm{X} 1$

$\mathrm{X} 1 \mathrm{~A}$

$\mathrm{X} 1 \mathrm{~B}$

$X 1 R$
$\operatorname{Im}\left[\beta_{1} a I_{0}(\alpha, a) I_{1}\left(\alpha_{2} a\right)-\beta_{2} a I_{0}\left(\alpha_{2} a\right) I_{1}(\alpha, a)\right]$

$\operatorname{Im}\left[\beta_{2} a I_{0}\left(\alpha_{2} a\right) K_{1}(\alpha, a)+\beta_{1} a K_{0}(\alpha, a) I_{1}\left(\alpha_{2} a\right)\right]$

$\operatorname{Im}\left[\beta, b I_{0}(\alpha, b) K_{1}(\alpha b)+\alpha b K_{0}(\alpha b) I_{1}(\alpha, b)\right]$

$\operatorname{Im}\left(K_{1}(\alpha, r)\left[\beta, a I_{0}(\alpha, a) I_{1}\left(\alpha_{2} a\right)-\beta_{2} a I_{0}\left(\alpha_{2} a\right) I_{1}(\alpha, a)\right]+\right.$

$$
\left.I_{1}(\alpha, r)\left[\beta_{2} a I_{0}\left(\alpha_{2} a\right) K_{1}(\alpha, a)+\beta, a K_{0}(\alpha, a) I_{1}\left(\alpha_{2} a\right)\right]\right\}
$$

$\operatorname{Re}\left\{K_{1}\left(\alpha_{1} r\right)\left[\beta, a I_{0}(\alpha, a) I_{1}\left(\alpha_{2} a\right)-\beta_{2} a I_{0}\left(\alpha_{2} a\right) I_{1}(\alpha, a)\right\}+\right.$

$$
\left.I_{1}\left(\alpha_{1} r\right)\left[\beta_{2} a I_{0}\left(\alpha_{2} a\right) K_{1}(\alpha, a)+\beta_{1} a K_{0}(\alpha, a) I_{1}\left(\alpha_{2} a\right)\right]\right\}
$$

$\operatorname{Re}\left[\alpha b K_{0}(\alpha b) K_{1}(\alpha, b)-\beta, b K_{0}(\alpha, b) K_{1}(\alpha b)\right]$

$\operatorname{Re}\left[\beta_{1} a I_{0}(\alpha, a) I_{1}\left(\alpha_{2} a\right)-\beta_{2} a I_{0}\left(\alpha_{2} a\right) I_{1}(\alpha, a)\right]$

$\operatorname{Re}\left[\beta_{2} a I_{0}\left(\alpha_{2} a\right) K_{1}(\alpha, a)+\beta_{1} a K_{0}(\alpha, a) I_{1}\left(\alpha_{2} a\right)\right]$

$\operatorname{Re}\left[\beta, b I_{0}(\alpha, b) K_{1}(\alpha b)+\alpha b K_{0}(\alpha b) I_{1}(\alpha, b)\right]$

$d \alpha$

$\operatorname{Im}\left\{K_{1}(\alpha, b)\left[\beta, a I_{0}(\alpha, a) I_{1}\left(\alpha_{2} a\right)-\beta_{2} a I_{0}\left(\alpha_{2} a\right) I_{1}(\alpha, a)\right]+\right.$

$\left.I_{1}(\alpha, b)\left\{\beta_{2} a I_{0}\left(\alpha_{2} a\right) K_{1}\left(\alpha_{1} a\right)+\beta_{1} a K_{0}\left(\alpha_{1} a\right) I_{1}\left(\alpha_{2} a\right)\right]\right\}$

$\operatorname{Re}\left[K_{1}(\alpha, b)\left[\beta, a I_{0}\left(\alpha_{1} a\right) I_{1}\left(\alpha_{2} a\right)-\beta_{2} a I_{0}\left(\alpha_{2} a\right) I_{1}(\alpha, a)\right]+\right.$ $\left.I_{1}(\alpha, b)\left[\beta_{2} a I_{0}\left(\alpha_{2} a\right) K_{1}(\alpha, a)+\beta_{1} a K_{0}\left(\alpha_{1} a\right) I_{1}\left(\alpha_{2} a\right)\right]\right\}$

$\operatorname{Re} \frac{1}{K_{1}(\alpha b)}\left[K_{1}(\alpha, b)\left[\beta, a I_{0}(\alpha, a) I_{1}\left(\alpha_{2} a\right)-\beta_{2} a I_{0}\left(\alpha_{2} a\right) I_{1}(\alpha, a)\right]+\right.$

$\left.I_{1}(\alpha, b)\left[\beta_{2} a I_{0}\left(\alpha_{2} a\right) K_{1}\left(\alpha_{1} a\right)+\beta_{1} a K_{0}\left(\alpha_{1} a\right) I_{1}\left(\alpha_{2} a\right)\right]\right]$

$\left[\left[\alpha b K_{0}(\alpha b) K_{1}(\alpha, b)-\beta, b K_{0}(\alpha, b) K_{1}(\alpha b)\right]\right.$

$\left[\beta, a I_{0}(\alpha, a) I_{1}\left(\alpha_{2} a\right)-\beta_{2} a I_{0}\left(\alpha_{2} a\right) I_{1}(\alpha, a)\right]+$

$\left[\beta_{2} a I_{0}\left(\alpha_{2} a\right) K_{1}\left(\alpha_{1} a\right)+\beta_{1} a K_{0}\left(\alpha_{1} a\right) I_{1}\left(\alpha_{2} a\right)\right]$

$\left[\left[\beta, b I_{0}(\alpha, b) K_{1}(\alpha b)+\alpha b K_{0}(\alpha b) I_{1}(\alpha, b)\right]\right]^{-1}-\frac{I_{1}(\alpha b)}{K_{1}(\alpha b)}$

$\alpha$

$\operatorname{Re}\left[\alpha_{1}\right]$

$\operatorname{Re}[\alpha, a]$

$\operatorname{Re}[\alpha, b]$

$\operatorname{Re}[\alpha, r]$ 


\begin{tabular}{|c|c|}
\hline XA & $\alpha a$ \\
\hline $\mathrm{XB}$ & $\alpha b$ \\
\hline $\mathrm{XF} 2$ & $\frac{2}{\pi} \frac{1}{\alpha^{3}} K\left(r_{2}, r_{1}\right) \sin (\alpha \ell / 2) d \alpha$ \\
\hline XFACT & $\frac{1}{\alpha^{3}} K\left(r_{2}, r_{1}\right) \sin (\alpha \ell / 2)$ \\
\hline XFACT2 & $\frac{2}{\pi} \frac{1}{\alpha^{3}} K\left(r_{2}, r_{1}\right) \sin (\alpha \ell / 2) \cos (\alpha z) d \alpha$ \\
\hline XJR21 & $\frac{1}{\alpha^{3}} J\left(r_{2}, r_{1}\right)$ \\
\hline XKR21 & $\frac{1}{\alpha^{3}} K\left(r_{2}, r_{1}\right)$ \\
\hline $\mathrm{XL}$ & $\alpha \ell$ \\
\hline $\mathrm{XX}$ & $\alpha^{2}$ \\
\hline $\mathrm{xxxx}$ & $\alpha^{4}$ \\
\hline $\mathrm{Y} 1$ & $\operatorname{Im}\left[\alpha_{1}\right]$ \\
\hline Y1A & $\operatorname{Im}[\alpha, a]$ \\
\hline Y1B & $\operatorname{Im}[\alpha, b]$ \\
\hline Y1R & $\operatorname{Im}[\alpha, r]$ \\
\hline ZIOIA & $\operatorname{Im}\left[\alpha, a I_{0}(\alpha, a)\right]$ \\
\hline ZIOIB & $\operatorname{Im}\left[\alpha_{1} b I_{0}(\alpha, b)\right]$ \\
\hline ZIOIR & $\operatorname{Im}\left[\alpha, r I_{0}(\alpha, r)\right]$ \\
\hline ZIORA & $\operatorname{Re}\left[\alpha, a I_{0}(\alpha, a)\right]$ \\
\hline ZIORB & $\operatorname{Re}\left[\alpha_{1} b I_{n}(\alpha, b)\right]$ \\
\hline ZIORR & $\operatorname{Re}\left[\alpha_{1} r I_{0}\left(\alpha_{1} r\right)\right]$ \\
\hline ZKOIA & $\operatorname{Im}\left[\alpha, a K_{0}(\alpha, a)\right]$ \\
\hline ZKOIB & $\operatorname{Im}\left[\alpha, b K_{0}(\alpha, b)\right]$ \\
\hline ZKOIR & $\operatorname{Im}\left[\alpha_{1} r K_{0}\left(\alpha_{1} r\right)\right]$ \\
\hline ZKOKA & $\operatorname{Re}\left[\alpha, a K_{0}(\alpha, a)\right]$ \\
\hline ZKORB & $\operatorname{Re}\left[\alpha, b K_{0}(\alpha, b)\right]$ \\
\hline ZKORR & $\operatorname{Re}\left[\alpha, r K_{0}(\alpha, r)\right]$ \\
\hline
\end{tabular}




\section{Sample output}

Output sent to printer:

ABENCAR TIME 6:34:51 DATE 8/18/89

IN RAD OT RAD LENGTH RAD CLR WALLTH \& WALL TUB IR TUB OR

$\begin{array}{lllllllll}\text { ACT } & 1.7750 & 2.0670 & 0.2650 & 0.0075 & 0.2200 & 45.45 & 1.5475 & 1.7675\end{array}$

$\begin{array}{lllllllll}\text { NOR } & 0.9240 & 1.0760 & 0.1379 & 0.0039 & 0.1145 & 45.45 & 0.8056 & 0.9201\end{array}$

RBAR $1.9210 \mathrm{FREQ}=4.000000 \mathrm{E}+02 \mathrm{RHO}=3.8400 \mathrm{PERM}=1.000 \mathrm{WUSRR}=195.8126$ NORM IMPD:RL 0.135056 IM 0.494500 AIR IND $1.826299 \mathrm{E}-02$

NORM DSF:RL 2.8462E-04 IM-1.0838E-04 VOLN 1.1079E-04

MAXIMUM MAG $0.1845 \mathrm{D}-03$ PHA AT MAX MAG 87.61 OD DEFECT

Partial listing of output defect axial position (ZD), defect magnitude (DFM) and defect phase (DFP) that is sent to LOD file (either FORT39 or ENCSCN.DAT):
0.000
$0.1845 D-03$
87.61
0.010
$0.1832 \mathrm{D}-03$
87.43
0.020
$0.1793 \mathrm{D}-03$
86.87
0.030
0.1729D-03
85.89
0.040
$0.1642 \mathrm{D}-03$
84.46
0.050
$0.1537 \mathrm{D}-03$
82.54
0.060
$0.1420 \mathrm{D}-03$
80.14
0.070
$0.1296 \mathrm{D}-03$
77.30
0.080
$0.1172 \mathrm{D}-03$
0.090
$0.1052 \mathrm{D}-03$
74.09
0.100
$0.9405 \mathrm{D}-04$
70.61
66.98

The program also plots the output on the CRT, and a plot similar to Fig: 17 can be obtained. The phases and magnitudes given in this report are referenced to the $X$ axis being zero phase and measured counterclockwise from $X$ axis, which is standard for mathematics and electrical engineering. Standard eddy-current practice is to measure the phase shift in a clockwise direction from the $-X$ axis. Furthermore, the phase is rotated until the probe wobble/fill factor/lift-off variations lie in a horizontal direction. 


\section{Listing}

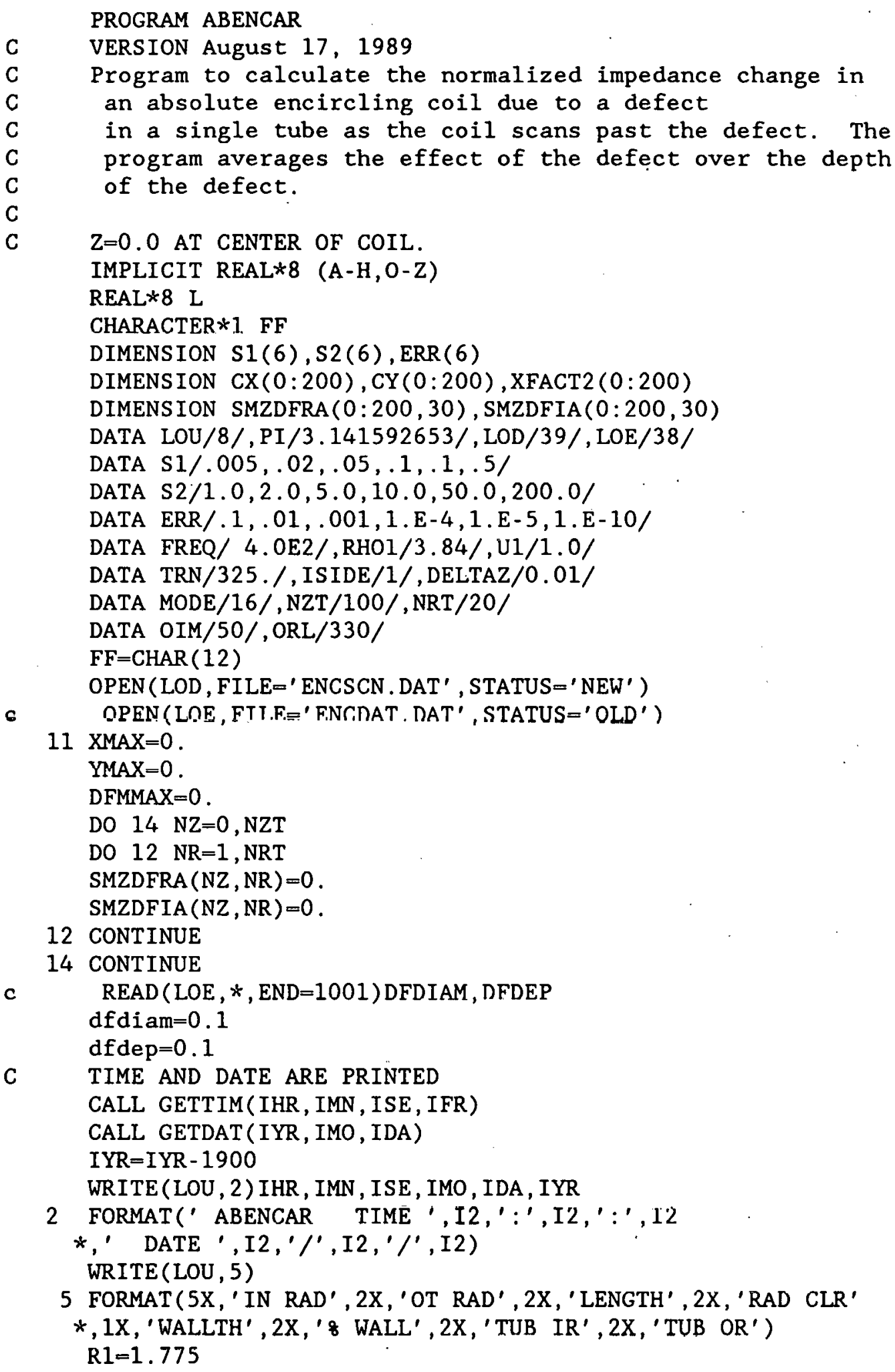




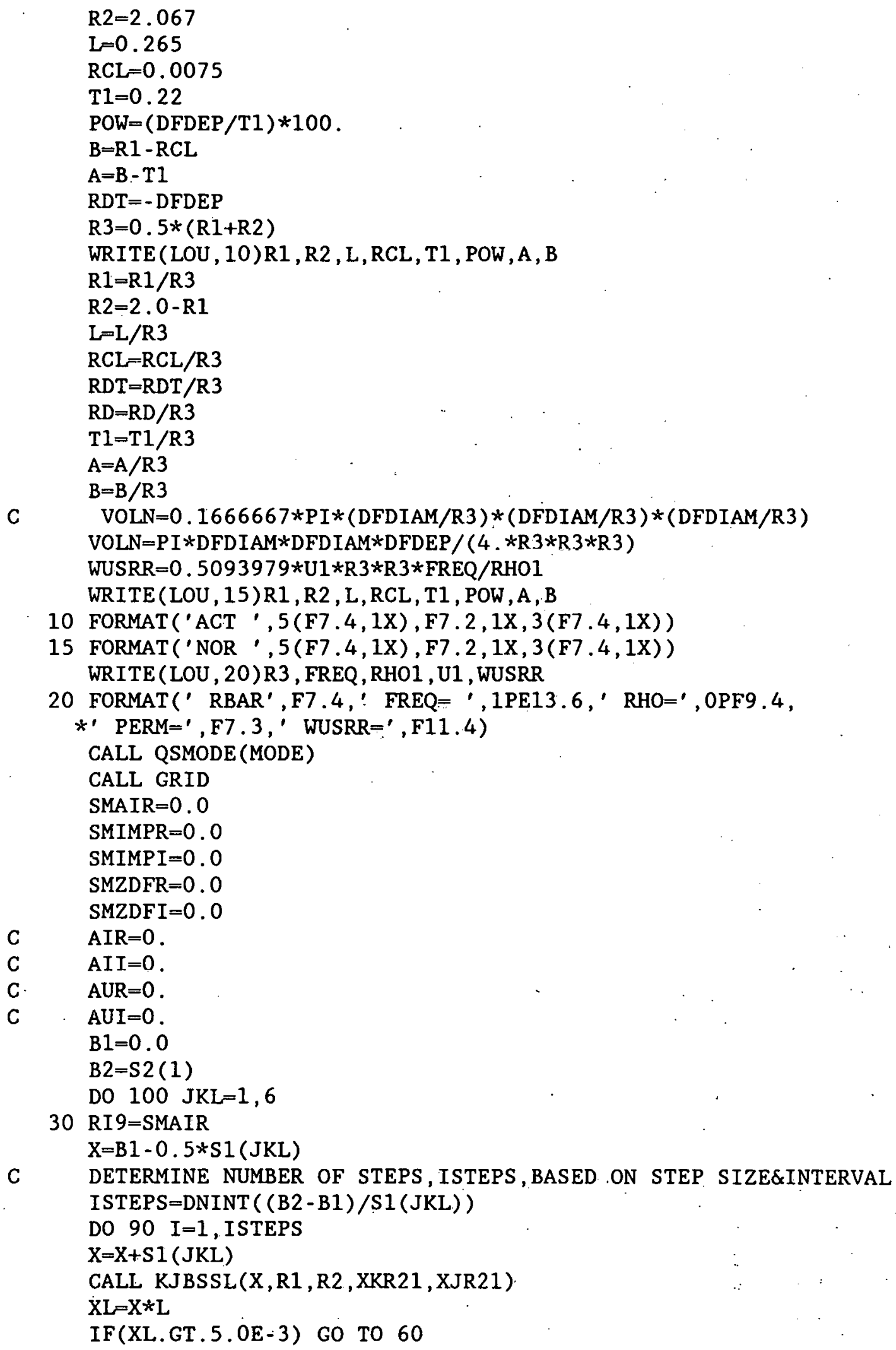




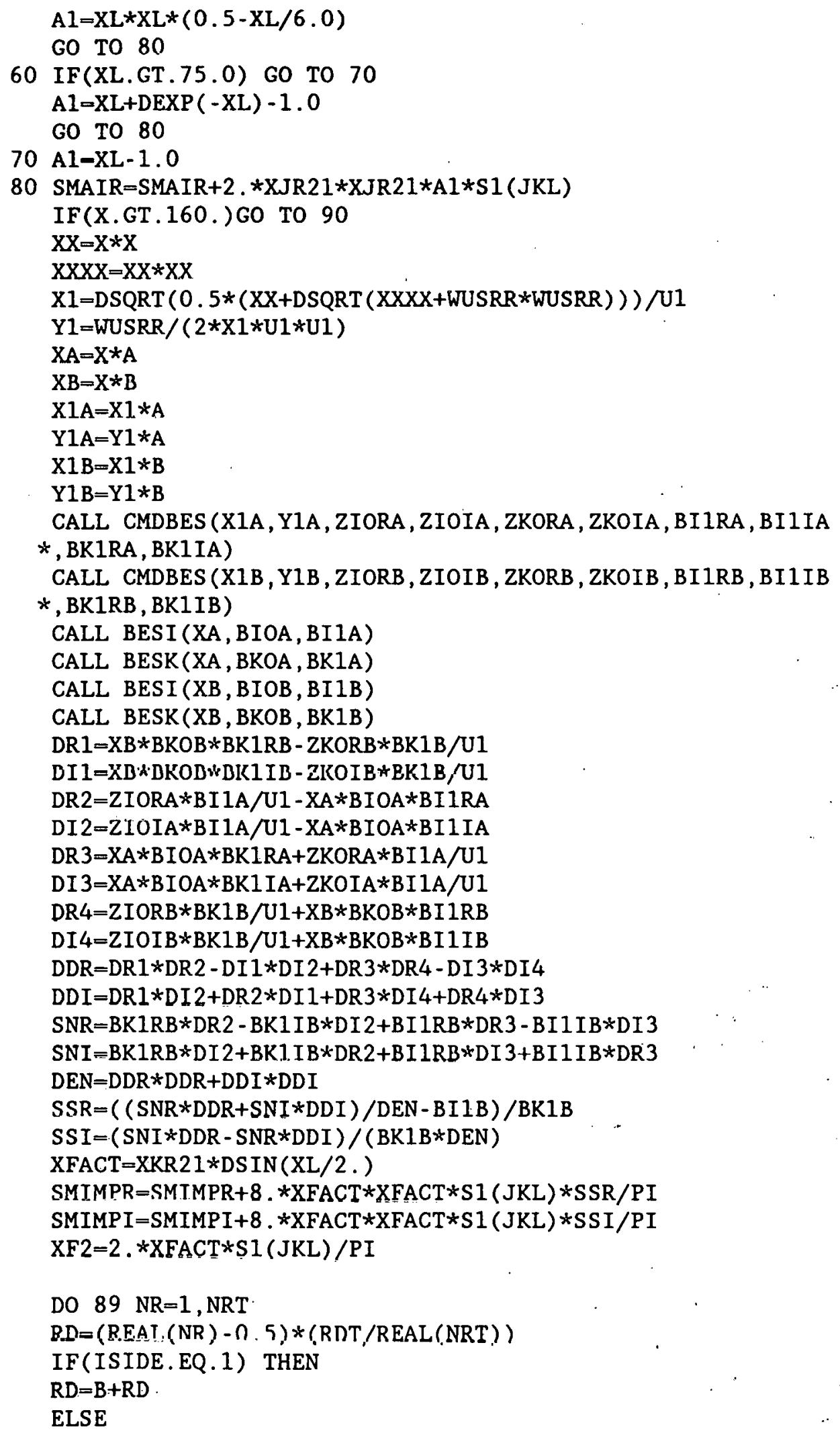




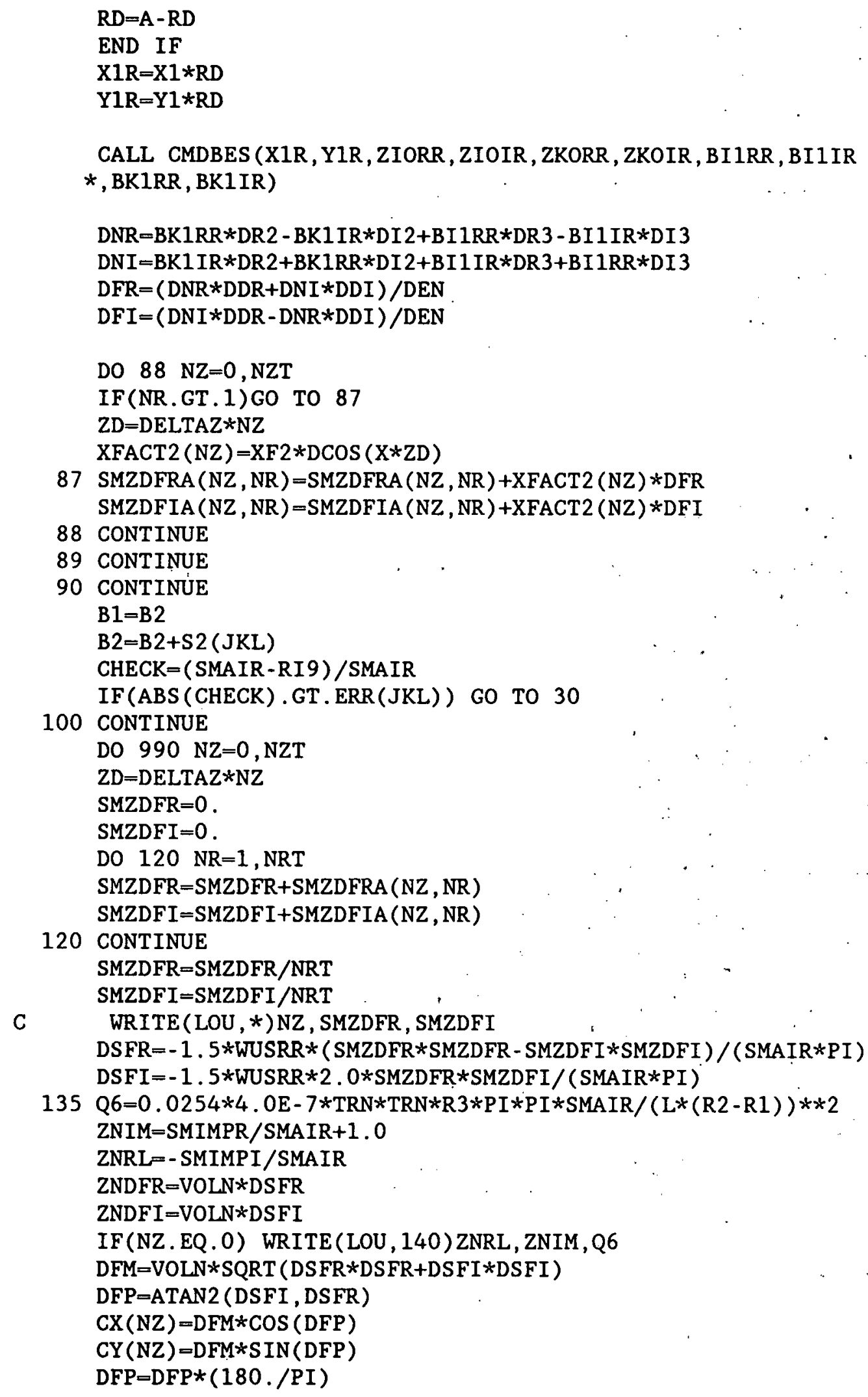




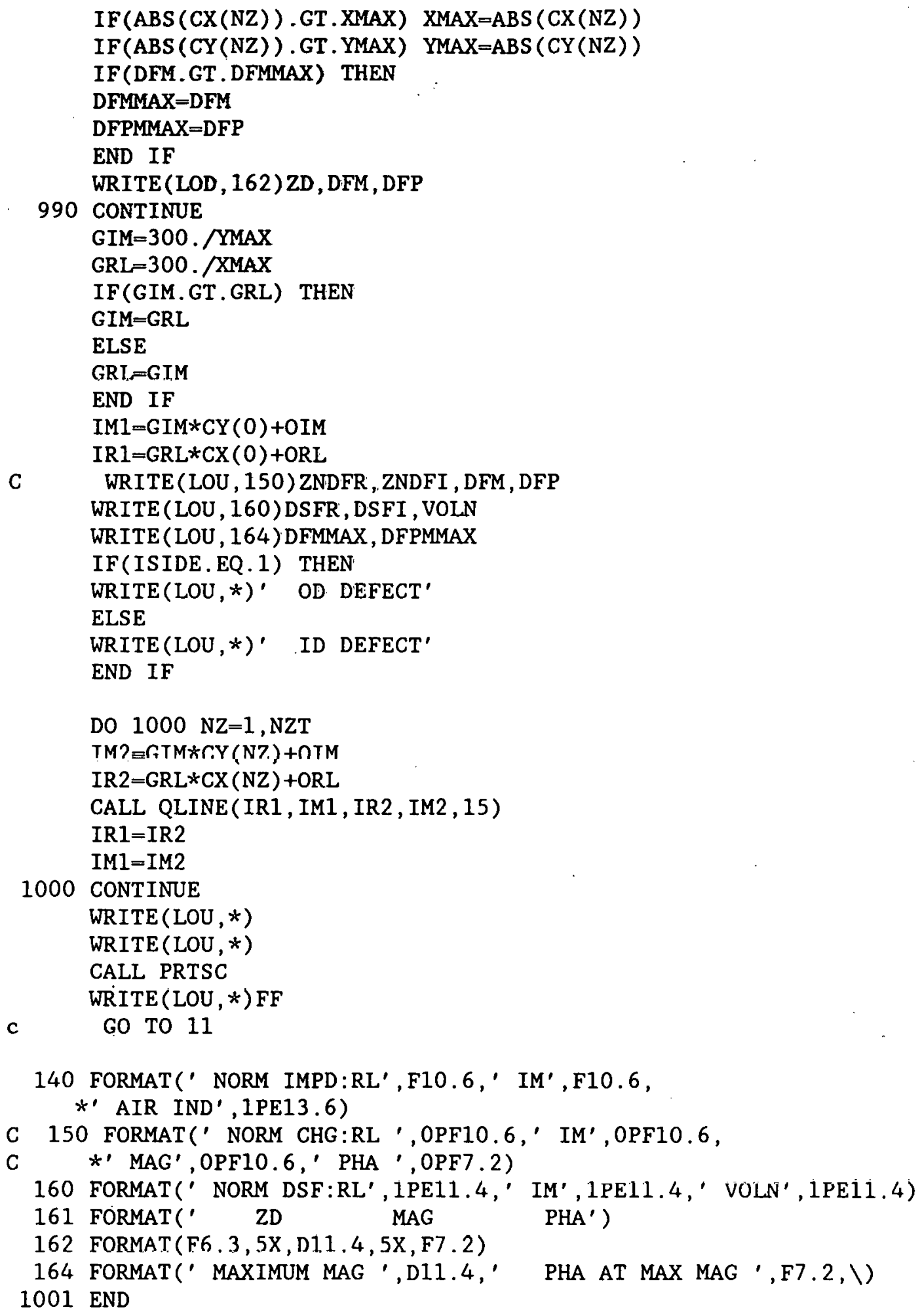


DFENCAR calculates defect impedance change, average over depth

Program DFENCAR calculates the change in the normalized impedance "of a differential encircling coil due to the presence of a defect in a single conducting tube, as shown in Fig. 20.

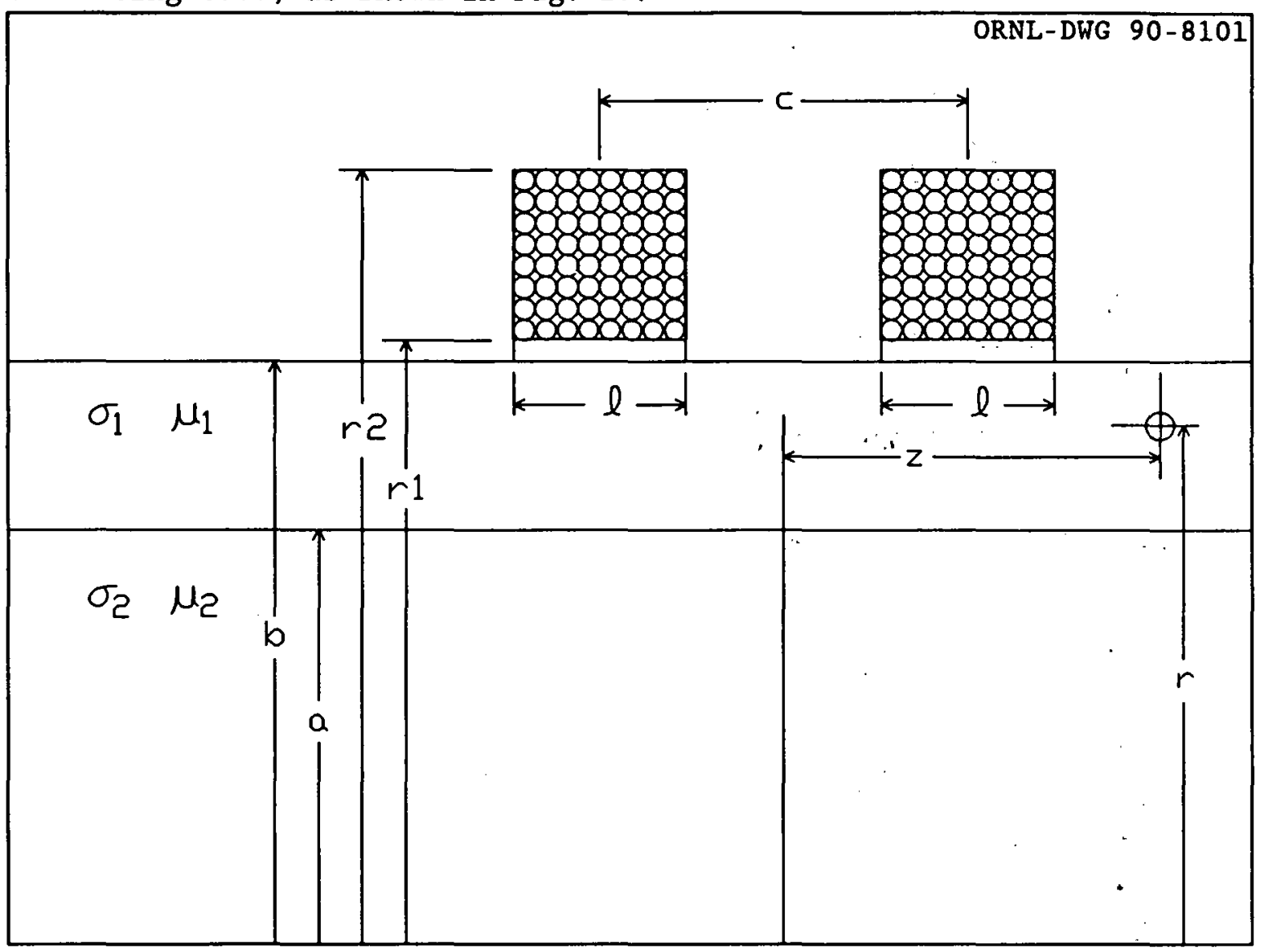

Fig. 20. Cross sectional view of a differential encircling probe.

The distance to the defect is measured from the center of the coil assembly, and the center-to-center distance of the coils is denoted $c$. The Impedance difference between the two matched coils is:

$$
Z_{\text {nd }}=Z_{1 \mathrm{~d}}-Z_{2 \mathrm{~d}}
$$

Substituting in from Éq. (29) for the impedance change of each coil and using some trigonometric identities gives for the normalized impedance difference: 


$$
\begin{aligned}
Z_{\mathrm{nd}}(r, z)=\frac{-3\left(\omega \mu \sigma_{1} \overline{\mathrm{r}}^{2}\right) V o I_{\mathrm{n}}}{2 \pi I_{\mathrm{a} \mid \mathrm{r}}} \\
\times\left[\int_{0}^{\infty} \frac{K\left(r_{2}, r_{1}\right)}{\pi \alpha^{3}}\left\{\frac{K_{1}\left(\alpha_{1} r\right) D_{2}+I_{1}\left(\alpha_{1} r\right) D_{3}}{\left(D_{1} D_{2}+D_{3} D_{4}\right)}\right\} \sin \left(\frac{\alpha \ell}{2}\right) \sin \left(\frac{\alpha c}{2}\right) \sin (\alpha z) 4 d \alpha\right] \\
\times\left[\int_{0}^{\infty} \frac{K\left(r_{2}, r_{1}\right)}{\pi \alpha^{3}}\left\{\frac{K_{1}\left(\alpha_{1} r\right) D_{2}+I_{1}\left(\alpha_{1} r\right) D_{3}}{\left(D_{1} D_{2}+D_{3} D_{4}\right)}\right\} \sin \left(\frac{\alpha \ell}{2}\right) \cos \left(\frac{\alpha c}{2}\right) \cos (\alpha z) 4 d \alpha\right]
\end{aligned}
$$

The definitions of the various terms in Eq. (55) are given in Eqs. (45) through (53) in the discussion of the absolute encircling coil. The program performs the calculations for a number of different axial distances between the center of the coil and the defect, and it can divide the defect into a number of parts and calculate the effect of each part separately.

\section{Summary}

1. Dimension arrays and declare variable types.

2. Initialize variables.

3. Begin the integration. Calculate the expressions that are independent of the position of the defect.

4. Select a value for the radial distance between the defect and the center of the coil. Do the calculations which depend only upon this component of the position.

5. Select a value for the axial distance between the defect and the center of the coil. Complete the calculations.

6. Loop to 5 until done.

7. Loop to 4 until done.

8. Output the results.

\section{Variables}

A number of the variables which occur mainly inside the integration loops do not correspond to anything physical. These variables are described in a section at the end. Starred variables must be assigned by the user.
A
B
The normalized inner radius of the tube.
C.
The normalized outer radius of the tube.
C. The axial distance between the centers of the two coils. The value is input in inches and
DELTAZ normalized by the program.
at which the calculations are done.
DFDEP ${ }^{\circ} \quad$ The depth of the defect in the tube in inches.
DFDIAM $\quad$ The diameter of the defect in the tube in inches. 
DFM

DFP

DSFI

DSFR

FREQ

ISIDE*

$L^{\circ}$

LOD $^{\circ}$

LOE*

LOU

$\mathrm{NRT}^{*}$

NZT*

POW

Q6

$\mathrm{R} 1^{\circ}$

R2

R3

RCL

RD

RDT

RHO1*

$\mathrm{T}^{\circ}$

TRN $^{*}$

$\mathrm{U} 1^{\circ}$

VOLN

WUSRR

The magnitude of the normalized impedance change in the coil due to the defect.

The phase of the normalized impedance change. in the coil due to the defect.

The imaginary part of the defect sensitivity factor of the coil.

The real part of the defect sensitivity factor of the coil.

The operating frequency in hertz.

The side of the tube where the defect is located. If ISIDE $=1$, the defect is on the outside of the tube; if ISIDE $=2$, the defect is on the inside of the tube.

The length of each coil. The value is input in inches and normalized by the program.

The number of the $I / O$ unit connected to the output data file.

The number of the $I / 0$ unit connected to a file which contains the diameters and depths of the defects for which calculations are to be performed.

The number of the $I / O$ unit connected to the printer.

The total number of parts centered along the axis of the defect into which the defect is divided to perform the calculations.

The total number of different values of the axial distance between the center of the coil and the defect for which calculations are performed.

The depth of the defect expressed as a percentage of wall thickness.

The inductance in henries of the coil in air.

The inner radius of each coil. The value is input in inches and normalized by the program.

The outer radius of each coil. The value is input in inches and normalized by the program.

The mean radius of each coil in inches.

The distance between the inside of the coil and the outside wall of the tube. The value is input in inches and normalized.by the program.

The radial distance between the center of the coil and the defect (see note 1).

The normalized depth to the bottom of the defect. A negative number.

The electrical resistivity of the tube in $\mu \Omega-\mathrm{cm}$.

The thickness of the tube wall. The value is input in inches and normalized by the program.

The number of turns in each coil.

The relative magnetic permeability of the tube.

The normalized volume of the defect.

The product of the angular operating frequency, 
the magnetic permeability of the tube, the electrical conductivity of the tube, and the square of the mean radius of the coil.

The axial distance between the center of the coil and the defect.

ZNDFI

The imaginary part of the normalized impedance change in the coil due to the defect.

ZNDFR The real part of the normalized impedance change in the coil due to the defrect..

ZNIM The imaginary part of the normalized impedance of the coil when no defects are present.

ZNRL The real part of the normalized impedance of the coil when no defects are present. 


\section{Integration section of Program DFENCAR}

\section{Symbol definitions}

The following are definitions of the symbols used to describe the program variables which appear in the integration section of the program. All lengths are normalized unless otherwise noted.

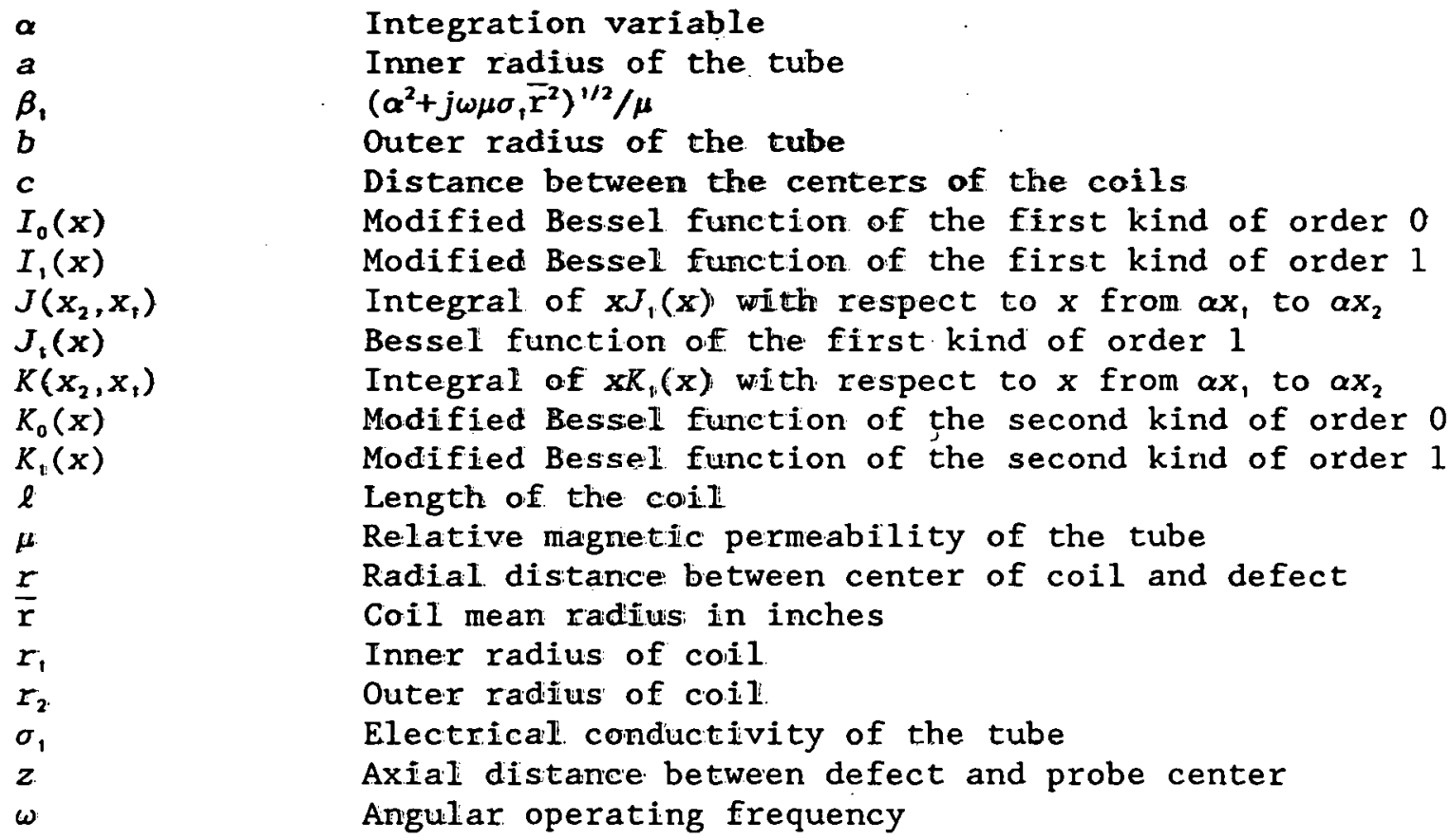

Variables appearing in the integration section

\begin{tabular}{|c|c|}
\hline $\begin{array}{l}\text { Program } \\
\text { variable }\end{array}$ & $\begin{array}{l}\text { Symbolic } \\
\text { equivalent }\end{array}$ \\
\hline A1 & $\alpha \ell+\exp (-\alpha \ell)-1$ \\
\hline BIOA & $I_{0}(\alpha a)$ \\
\hline BIOB & $I_{0}(\alpha b)$ \\
\hline BIIA & $I_{1}(\alpha a)$ \\
\hline BI $1 B$ & $I_{1}(\alpha b)$ \\
\hline BIIIA & $\operatorname{Im}\left[I_{1}(\alpha, a)\right]$ \\
\hline BIIIB & $\operatorname{Im}\left[I_{1}\left(\alpha_{1} b\right)\right]$ \\
\hline BIIIR & $\operatorname{Im}\left[I_{1}(\alpha, r)\right]$ \\
\hline BI1RA & $\operatorname{Re}\left[I_{1}(\alpha, a)\right]$ \\
\hline BIIRB & $\operatorname{Re}\left[I_{1}(\alpha, b)\right]$ \\
\hline
\end{tabular}




\begin{tabular}{|c|c|}
\hline BIIRR & $\operatorname{Re}\left[I_{1}(\alpha, r)\right]$ \\
\hline BKOA & $K_{0}(\alpha a)$ \\
\hline $\mathrm{BKOB}$ & $K_{0}(\alpha b)$ \\
\hline BK1A & $K_{1}(\alpha a)$ \\
\hline BK1B & $K_{1}(\alpha b)$ \\
\hline BK1IA & $\operatorname{Im}[K,(\alpha, a)]$ \\
\hline BK1IB & $\operatorname{Im}\left[K_{1}(\alpha, b)\right]$ \\
\hline BK1IR & $\operatorname{Im}\left[K_{1}(\alpha, r)\right]$ \\
\hline BK1RA & $\operatorname{Re}\left[K_{1}\left(\alpha_{1} a\right)\right]$ \\
\hline BKIRB & $\operatorname{Re}\left[K_{1}(\alpha, b)\right]$ \\
\hline BK1RR & $\operatorname{Re}\left[K_{1}\left(\alpha_{1} r\right)\right]$ \\
\hline \multirow[t]{4}{*}{ DDI } & $\operatorname{Im}\left(\left[\alpha b K_{0}(\alpha b) K_{1}(\alpha, b)-\beta, b K_{0}(\alpha, b) K_{1}(\alpha b)\right]\right.$ \\
\hline & {$\left[\beta, a I_{0}(\alpha, a) I_{1}\left(\alpha_{2} a\right)-\beta_{2} a I_{0}\left(\alpha_{2} a\right) I_{1}(\alpha, a)\right]+$} \\
\hline & {$\left[\beta_{2} a I_{0}\left(\alpha_{2} a\right) K_{1}(\alpha, a)+\beta_{1} a K_{0}(\alpha, a) I_{1}\left(\alpha_{2} a\right)\right]$} \\
\hline & $\left.\left[\beta, b I_{0}(\alpha, b) K_{1}(\alpha b)+\alpha b K_{0}(\alpha b) I_{1}(\alpha, b)\right]\right\}$ \\
\hline \multirow[t]{4}{*}{ DDR } & $\operatorname{Re}\left\{\left[\alpha b K_{0}(\alpha b) K_{1}(\alpha, b)-\beta, b K_{0}(\alpha, b) K_{1}(\alpha b)\right]\right.$ \\
\hline & {$\left[\beta_{1} a I_{0}(\alpha, a) I_{1}\left(\alpha_{2} a\right)-\beta_{2} a I_{0}\left(\alpha_{2} a\right) I_{1}\left(\alpha_{1} a\right)\right]+$} \\
\hline & {$\left[\beta_{2} a I_{0}\left(\alpha_{2} a\right) K_{1}(\alpha, a)+\beta, a K_{0}(\alpha, a) I,\left(\alpha_{2} a\right)\right]$} \\
\hline & $\left.\left[\beta, b I_{0}(\alpha, b) K_{1}(\alpha b)+\alpha b K_{0}(\alpha b) I_{1}(\alpha, b)\right]\right\}$ \\
\hline \multirow[t]{6}{*}{ DFR } & $\operatorname{Re}\left\{K_{1}(\alpha, r)\left[\beta, a I_{0}(\alpha, a) I_{1}\left(\alpha_{2} a\right)-\beta_{2} a I_{0}\left(\alpha_{2} a\right) I_{1}(\alpha, a)\right\}+\right.$ \\
\hline & $\left.I_{1}(\alpha, r)\left[\beta_{2} a I_{0}\left(\alpha_{2} a\right) K_{1}\left(\alpha_{1} a\right)+\beta_{1} a K_{0}\left(\alpha_{1} a\right) I_{1}\left(\alpha_{2} a\right)\right]\right\}$ \\
\hline & $\div\left(\left[\alpha b K_{0}(\alpha b) K_{1}(\alpha, b)-\beta, b K_{0}(\alpha, b) K_{1}(\alpha b)\right]\right.$ \\
\hline & {$\left[\beta, a I_{0}(\alpha, a) I_{1}\left(u_{2} a\right)-\beta_{2} a I_{0}\left(\alpha_{2} a\right) I_{1}\left(\alpha_{1} a\right)\right]+$} \\
\hline & {$\left[\beta_{2} a I_{0}\left(\alpha_{2} a\right) K,\left(\alpha_{1} a\right)+\beta_{1} a K_{0}\left(\alpha_{1} a\right) I_{1}\left(\alpha_{2} a\right)\right]$} \\
\hline & $\left.\left[\beta, b I_{0}(\alpha, b) K_{1}(\alpha b)+\alpha b K_{0}(\alpha b) I_{1}(\alpha, b)\right]\right\}$ \\
\hline DI1 & $\operatorname{Im}\left[\alpha b K_{0}(\alpha b) K_{1}(\alpha, b)-\beta_{t} b K_{0}(\alpha, b) K_{\mathfrak{t}}(\alpha b)\right]$ \\
\hline DI2 & $\operatorname{Im}\left[\beta_{1} a I_{0}(\alpha, a) I_{1}\left(\alpha_{2} a\right)-\beta_{2} a I_{0}\left(\alpha_{2} a\right) I_{1}\left(\alpha_{1} a\right)\right]$ \\
\hline DI 3 & $\operatorname{Im}\left[\beta_{2} a I_{0}\left(\alpha_{2} a\right) K_{1}(\alpha, a)+\beta_{1} a K_{0}(\alpha, a) I_{1}\left(\alpha_{2} a\right)\right]$ \\
\hline DI4 & $\operatorname{Im}\left[\beta, b I_{0}(\alpha, b) K_{1}(\alpha b)+\alpha b K_{0}(\alpha b) I_{1}(\alpha, b)\right]$ \\
\hline
\end{tabular}


DNI

DNR

DRI

DR2

DR3

DR 4

S1

SNI

SNR

SSR

$\mathrm{X}$

$\mathrm{X} 1$

$\mathrm{X} 1 \mathrm{~A}$

$\mathrm{X} 1 \mathrm{~B}$

$\mathrm{X} 1 \mathrm{R}$

$\mathrm{X} A$

$\mathrm{XB}$

XFACT
$\operatorname{Im}\left\{K_{1}(\alpha, r)\left[\beta, a I_{0}(\alpha, a) I_{1}\left(\alpha_{2} a\right)-\beta_{2} a I_{0}\left(\alpha_{2} a\right) I_{1}(\alpha, a)\right]+\right.$ $\left.I_{1}(\alpha, r)\left[\beta_{2} a I_{0}\left(\alpha_{2} a\right) K_{1}\left(\alpha_{i} a\right)+\beta_{1} a K_{0}\left(\alpha_{1} a\right) I_{i}\left(\alpha_{2} a\right)\right]\right\}$

$\operatorname{Re} \mid K_{1}(\alpha, r)\left[\beta, a I_{0}(\alpha, a) I_{1}\left(\alpha_{2} a\right)-\beta_{2} a I_{0}\left(\alpha_{2} a\right) I_{1}(\alpha, a)\right]+$ $\left.I_{1}(\alpha, r)\left[\beta_{2} a I_{0}\left(\alpha_{2} a\right) K_{1}(\alpha, a)+\beta_{1} a K_{0}(\alpha, a) I_{1}\left(\alpha_{2} a\right)\right]\right)$

$\operatorname{Re}\left[\alpha b K_{0}(\alpha b) K_{1}\left(\alpha_{1} b\right)-\beta_{1} b K_{0}(\alpha, b) K_{1}(\alpha b)\right]$

$\operatorname{Re}\left[\beta, a I_{0}(\alpha, a) I_{1}\left(\alpha_{2} a\right)-\beta_{2} a I_{0}\left(\alpha_{2} a\right) I_{1}(\alpha, a)\right]$

$\operatorname{Re}\left[\beta_{2} a I_{0}\left(\alpha_{2} a\right) K_{1}\left(\alpha_{1} a\right)+\beta_{1} a R_{0}\left(\alpha_{1} a\right) I_{1}\left(\alpha_{2} a\right)\right]$

$\operatorname{Re}\left[\beta, b I_{0}(\alpha, b) K_{1}(\alpha b)+\alpha b K_{0}(\alpha b) I_{1}(\alpha, b)\right]$

$d \alpha$

$\operatorname{Im}\left\{K_{1}\left(\alpha_{1} b\right)\left[\beta_{1} a I_{0}\left(\alpha_{1} a\right) I_{1}\left(\alpha_{2} a\right)-\beta_{2} a I_{0}\left(\alpha_{2} a\right) I_{1}\left(\alpha_{1} a\right)\right]+\right.$

$\left.I_{1}(\alpha, b)\left[\beta_{2} a I_{0}\left(\alpha_{2} a\right) K_{1}(\alpha, a)+\beta_{1} a K_{0}(\alpha, a) I_{1}\left(\alpha_{2} a\right)\right]\right\}$

$\operatorname{Re}\left[\mathcal{K}_{1}\left(\alpha_{1} b\right)\left[\beta_{1} a I_{0}\left(\alpha_{1} a\right) I_{1}\left(\alpha_{2} a\right)-\beta_{2} a I_{0}\left(\alpha_{2} a\right) I_{1}(\alpha, a)\right]+\right.$

$\left.I_{1}\left(\alpha_{1} b\right)\left[\beta_{2} a I_{0}\left(\alpha_{2} a\right) K_{1}\left(\alpha_{1} a\right)+\beta_{1} a K_{0}\left(\alpha_{1} a\right) I_{1}\left(\alpha_{2} a\right)\right]\right\}$

$\operatorname{Re} \frac{1}{K_{n}(\alpha, b)}\left[K_{t}\left(\alpha_{1} b\right)\left[\beta_{1} a I_{0}\left(\alpha_{1} a\right) I_{1}\left(\alpha_{2} a\right)-\beta_{2} a I_{0}\left(\alpha_{2} a\right) I_{1}(\alpha, a)\right]+\right.$

$\left.\left.I_{11}\left(\alpha_{1} b\right) \| \beta_{2} a I_{10}\left(\alpha_{2} a\right) K_{1}\left(\alpha_{11} a\right)+\beta_{1} a K_{0}(\alpha, a) I_{1}\left(\alpha_{2} a\right)\right]\right]$

$\left[\left[\alpha b K_{0}(\alpha b) K_{11}\left(\alpha_{11} b\right)-\beta, b K_{0}\left(\alpha_{1} b\right) K_{1}(\alpha b)\right]\right.$

$\left[\beta_{11} a I_{0}\left(\alpha_{1} a\right) I_{1}\left(\alpha_{2} a\right)-\beta_{2} a I_{0}\left(\alpha_{2} a\right) I_{1}(\alpha, a)\right]+$

$\left[\beta_{2} a I_{0}\left(\left(\alpha_{2} a\right) K_{11}(\alpha, a)+\beta_{1} a K_{0} \cdot\left(\alpha_{1} a\right) I_{1}\left(\alpha_{2} a\right)\right]\right.$

$\left[\left[\beta_{1} b I_{0}(\alpha, b) K_{1}(\alpha b)+\alpha b K_{0}(\alpha b) I_{1}(\alpha, b)\right]\right]^{-1}-\frac{I_{1}(\alpha b)}{K_{1}(\alpha b)}$

$\alpha$

$\operatorname{Re}\left[\boldsymbol{\alpha}_{1}\right]$

$\operatorname{Re}[\alpha, a]$

$\operatorname{Re}\left[\left[\alpha_{i} b\right]\right.$

$\operatorname{Re}[\alpha, r]$

$\alpha a$

$\alpha b$

$\frac{1}{\alpha^{3}} K\left(r_{2}, r_{1}\right) \sin (\alpha \ell / 2)$ 


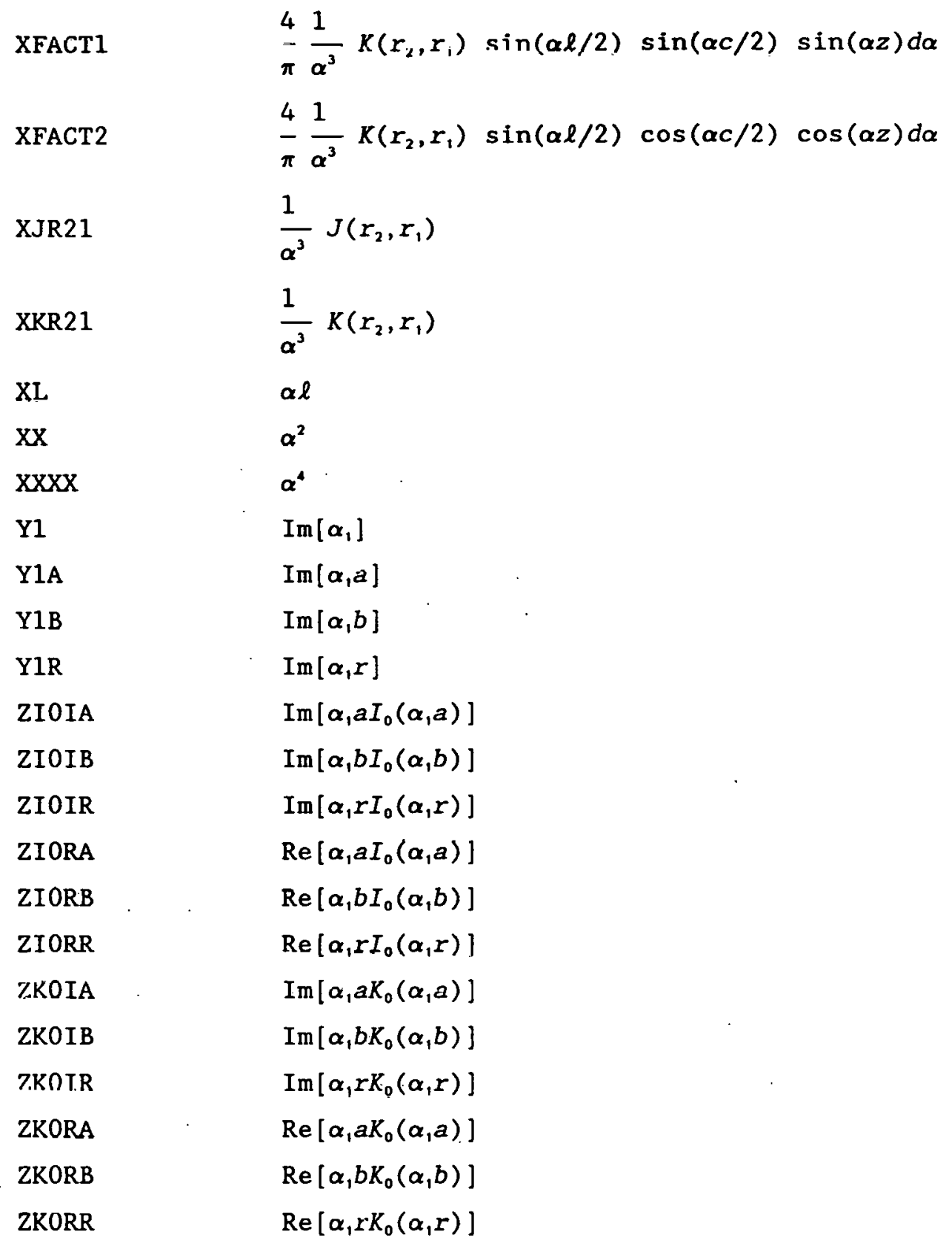

\section{Sample output}

A sample of the printer output is given below:

DFENCAR TIME 11:32: 0 DATE 8/18/89

IN RAD OT RAD LENGTH RAD CLR WALLTH \& WALL C TO C TUB IR TUB OR $\begin{array}{llllllllll}\text { ACT } & 1.7750 & 2.0670 & 0.2650 & 0.0075 & 0.2200 & 45.45 & 0.5150 & 1.5475 & 1.7675\end{array}$ $\begin{array}{lllllllll}\text { NOR. } 0.9240 & 1.0760 & 0.1379 & 0.0039 & 0.1145 & 45.45 & 0.2681 & 0.8056 & 0.9201\end{array}$ RBAR 1.9210 FREQ $=4.000000 E+02 \mathrm{RHO}=3.8400 \mathrm{PERM}=1.000 \mathrm{WUSRR}=195.8126$ NORM IMPD:RL 0.135056 IM 0.494500 AIR IND 1.826299E-02 
NORM DSF:RL 3.3340E-04 IM-2.1253E-04 VOLN 1.1079E-04

MAXIMUM MAG 0.1826D-03 PHA AT MAX MAG 91.17 OD DEFECT

Partial listing of output defect axial position (ZD), defect magnitude (DFM) and defect phase (DFP) that is sent to LOD file (either FORT39 or ENCSCN.DAT):

$\begin{array}{ccc}0.010 & 0.1723 \mathrm{D}-04 & 0.8304 \mathrm{D}+02 \\ 0.020 & 0.3455 \mathrm{D}-04 & 0.8350 \mathrm{D}+02 \\ 0.030 & 0.5201 \mathrm{D}-04 & 0.8425 \mathrm{D}+02 \\ 0.040 & 0.6962 \mathrm{D}-04 & 0.8524 \mathrm{D}+02 \\ 0.050 & 0.8728 \mathrm{D}-04 & 0.8641 \mathrm{D}+02 \\ 0.060 & 0.1047 \mathrm{D}-03 & 0.8766 \mathrm{D}+02 \\ 0.070 & 0.1216 \mathrm{D}-03 & 0.8888 \mathrm{D}+02 \\ 0.080 & 0.1373 \mathrm{D}-03 & 0.8996 \mathrm{D}+02 \\ 0.090 & 0.1513 \mathrm{D}-03 & 0.9083 \mathrm{D}+02 \\ 0.100 & 0.1631 \mathrm{D}-03 & 0.9144 \mathrm{D}+02 \\ \ldots \ldots & & \end{array}$

If the proper plotting software has been installed, a plot of these data is made on the CRT and a hard copy can be obtained, as shown in Fig. 21. The plot forms one-half the normal Lissajous pattern one gets in an eddycurrent test with a differential bobbin coil. The second half can be obtained by reflecting the signal in the $-z$ direction, since the signal is anti-symmetric. The phases and magnitudes given in this report are referenced to the $X$ axis being zero phase and measured counterclockwise from $X$ axis, which is standard for mathematics and electrical engineering. Standard eddy-current practice is to measure the phase shift in a clockwise direction from the $-X$ axis. Furthermore, the phase is rotated until the probe wobble/fill factor/lift-off variations 1 ie in a horizontal direction. 


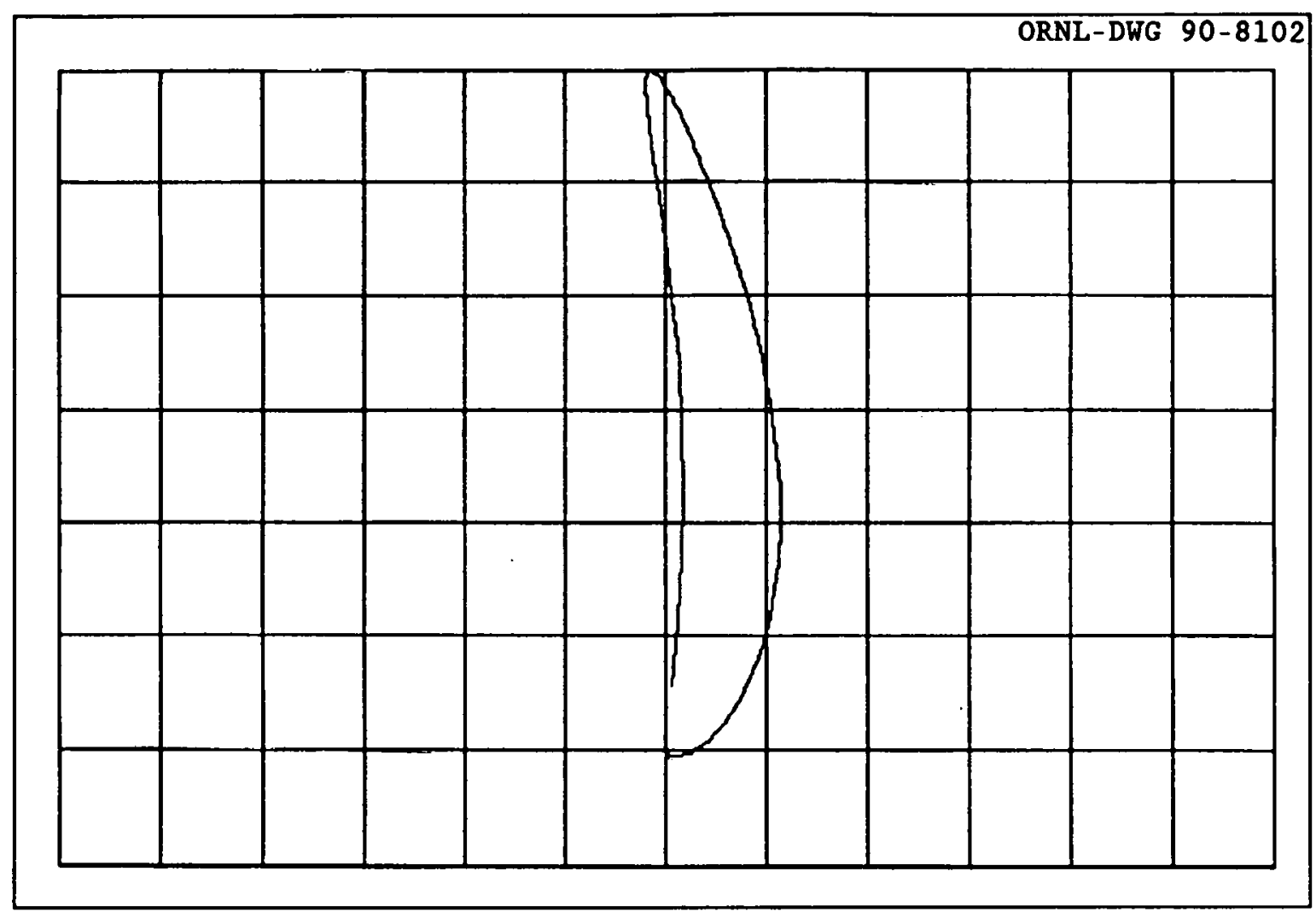

Fig. 21. Plot of defect signal on complex impedance plane as the defect is moved from the probe center in the plus $z$ direction. 


\section{Listing}

\section{PROGRAM DFENCAR}

C VERSION July 11,1988

C PROGRAM TO CALCULATE THE NORMALIZED IMPEDANCE CHANGE

C FOR A DEFECT IN A SINGLE TUBE WITH AN ENCIRCLING COIL

C $Z=0.0$ AT CENTER OF COIL.

IMPLICIT REAL*8 (A-H,O-Z)

REAL $* 8$ L

CHARACTER $* 1$ FF.

DIMENSION $S 1(6), S 2(6), \operatorname{ERR}(6)$

DIMENSION CX(200), CY(200)

DIMENSION SMZDFRIA $(200,30), \operatorname{SMZDFIIA}(200,30)$

DIMENSION SMZDFR2A $(200,30), \operatorname{SMZDFI} 2 A(200,30)$

DATA LOU/8/,PI/3.141592653/,LOD/39/,LOE/38/

DATA $S 1 / .005, .02, .05, .1, .1, .5 /$

DATA $S 2 / 1.0,2.0,5.0,10.0,50.0,200.0 /$

DATA ERR/.1,.01,.001,1.E-4,1.E-5,1.E-10/

DATA FREQ/ 4.0E2/,RHO1/3.84/,U1/1.0/

DATA TRN $/ 325 . /$, ISIDE $/ 1 /$,DELTAZ $/ 0.01 /$

DATA MODE $/ 16 /$, NZT $/ 100 /$, NRT $/ 20 /$

DATA OIM $/ 50 /, 0 R L / 330 /$

$F F=$ CHAR (12)

C OPEN(LOD, FILE=' ENCSCN.DAT' , STATUS = ' NEW' )

OPEN (LOE , FILE =' ENCDAT . DAT' , STATUS $=$ ' OLD' )

$11 \mathrm{XMAX}=0$.

YMAX $=0$.

DFMMAX $=0$.

DO $14 \mathrm{NZ}=1$, NZT

DO $12 \mathrm{NR}=1$, NRT

SMZDFR1A (NZ, NR $)=0$.

$\operatorname{SMZDFIIA}(\mathrm{NZ}, \mathrm{NR})=0$.

$\operatorname{SMZDFR} 2 A(N Z, N R)=0$.

12 CONTINUE

SMZDFI2A (NZ, NR $)=0$.

14 CONTINUE

READ (LOE , * END=1001).DFDIAM, DFDEP

C

TIME AND DATE ARE PRINTED

CALL GETTIM(IHR, IMN, ISE, IFR)

CALL GETDAT (IYR, IMO, IDA)

$I Y R=I Y R-1900$

WRITE (LOU , 2) IHR, IMN, ISE, IMO, IDA, IYR

2 FORMAT(' DFENCAR TIME ', I2,' :', I2, ' :' , I2

*,' DATE ', I2,'/', I2,' /, I2)

WRITE (LOU , 5)

5 FORMAT (5X,'IN RAD', $2 \mathrm{X}$, 'OT RAD', $2 \mathrm{X}$, 'LENGTH', $2 \mathrm{X}$, 'RAD CLR'

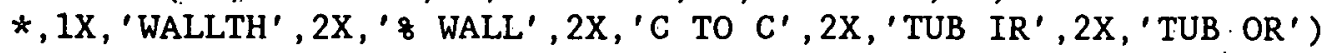

$\mathrm{R} 1=1.775$

$\mathrm{R} 2=2.067$

$\mathrm{L}=0.265$

$\mathrm{C}=0.515$ 


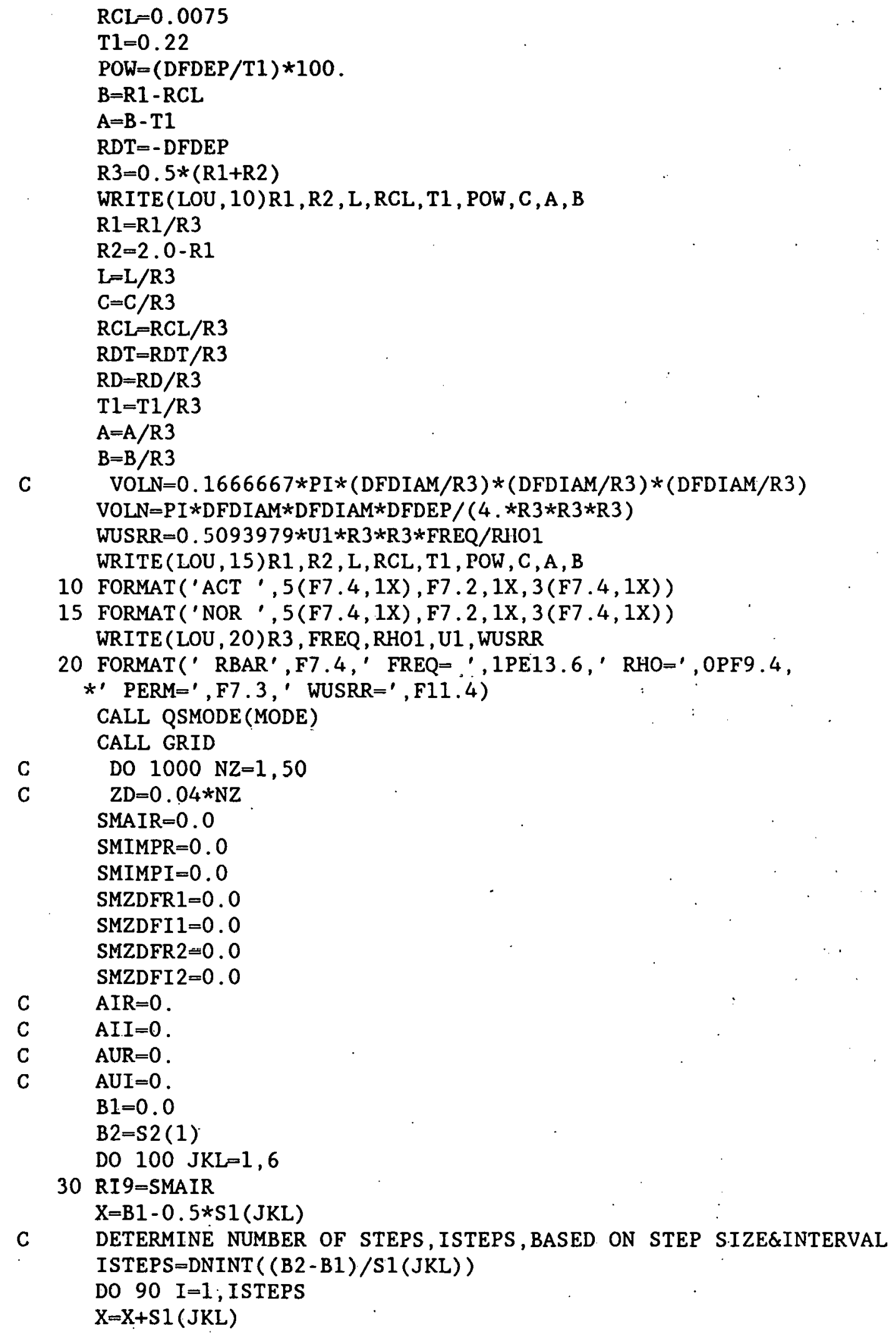


CALL KJBSSL(X,R1, R2, XKR21, XJR21)

$\mathrm{XL}=\mathrm{X} * \mathrm{~L}$

IF (XL.GT.5.0E - 3) GO TO 60

$\mathrm{Al}=\mathrm{XL} * \mathrm{XL} *(0.5-\mathrm{XL} / 6.0)$

GO TO 80

60 IF(XL.GT.75.0) GO TO 70

$\mathrm{Al}=\mathrm{XL}+\mathrm{DEXP}(-\mathrm{XL})-1.0$

GO TO 80

$70 \mathrm{~A} 1=\mathrm{XL}-1.0$

80 SMAIR=SMAIR $+2 . * X J R 21 * X J R 21 * A 1 * S 1(J K L)$

IF (X.GT.160.) GO TO 90

$\mathrm{XX}=\mathrm{X} * \mathrm{X}$

$\mathrm{XXXX}=\mathrm{XX} * \mathrm{XX}$

$\mathrm{X} 1=\mathrm{DSQRT}(0.5 *(\mathrm{XX}+\mathrm{DSQRT}(\mathrm{XXXX}+\mathrm{WUSRR} * \mathrm{WUSRR}))) / \mathrm{U} 1$

$\mathrm{Y} 1=\mathrm{WUSRR} /(2 * \mathrm{X} 1 * \mathrm{U} 1 * \mathrm{U} 1)$

$X A=X * A$

$\mathrm{XB}=\mathrm{X} * \mathrm{~B}$

$\mathrm{X} 1 \mathrm{~A}=\mathrm{X} 1 * \mathrm{~A}$

$Y 1 A=Y 1 * A$

$\mathrm{X} 1 \mathrm{~B}=\mathrm{X} 1 * \mathrm{~B}$

$\mathrm{Y} 1 \mathrm{~B}=\mathrm{Y} 1 * \mathrm{~B}$

CALL CMDBES (XIA, Y1A, ZIORA, ZIOIA, ZKORA, ZKOIA, BIIRA, BIIIA *, BKIRA, BKIIA)

CALL CMDBES (X1B , Y1B , ZIORB , ZIOIB , ZKORB , ZKOIB , BIIRB , BI1 IB

*, BK1RB, BK1IB)

CALL BESI (XA, BIOA, BIIA)

CALL BESK(XA, BKOA, BK1A)

CALL BESI (XB, BIOB, BI1B)

CALL BESK (XB,BKOB, BKIB)

$\mathrm{DR} 1=\mathrm{XB} * \mathrm{BKOB} * \mathrm{BK} 1 \mathrm{RB}-\mathrm{ZKORB} * \mathrm{BK} 1 \mathrm{~B} / \mathrm{U} 1$

$\mathrm{DI} 1=\mathrm{XB} * \mathrm{BKOB} * \mathrm{BK} 1 \mathrm{IB}-\mathrm{ZKOIB} * \mathrm{BK} 1 \mathrm{~B} / \mathrm{U} 1$

$\mathrm{DR} 2=\mathrm{ZIORA} * \mathrm{BI} 1 \mathrm{~A} / \mathrm{U} 1-\mathrm{XA} * \mathrm{BIOA} * \mathrm{BI} 1 \mathrm{RA}$

DI2 $=Z I O I A * B I 1 A / U 1-X A * B I O A * B I 1 I A$

$\mathrm{DR} 3=\mathrm{XA} * \mathrm{BIOA} * \mathrm{BK} 1 \mathrm{RA}+\mathrm{ZKORA} * \mathrm{BI} 1 \mathrm{~A} / \mathrm{U} 1$

$\mathrm{DI} 3=\mathrm{XA} * \mathrm{BIOA} * \mathrm{BK} 1 \mathrm{IA}+2 \mathrm{KOIA} * \mathrm{BI} 1 \mathrm{~A} / \mathrm{U} 1$

$\mathrm{DR} 4=\mathrm{ZIORB} * \mathrm{BK} 1 \mathrm{~B} / \mathrm{U} 1+\mathrm{XB} * \mathrm{BKOB} * \mathrm{BI} 1 \mathrm{RB}$

$\mathrm{DI} 4=\mathrm{ZIOIB} * \mathrm{BK} 1 \mathrm{~B} / \mathrm{U} 1+\mathrm{XB} * \mathrm{BKOB} * \mathrm{BI} 1 \mathrm{IB}$

$\mathrm{DDR}=\mathrm{DR} 1 * \mathrm{DR} 2-\mathrm{DI} 1 * \mathrm{DI} 2+\mathrm{DR} 3 * \mathrm{DR} 4-\mathrm{DI} 3 * \mathrm{DI} 4$

$\mathrm{DDI}=\mathrm{DR} 1 * \mathrm{DI} 2+\mathrm{DR} 2 * \mathrm{DI} 1+\mathrm{DR} 3 * \mathrm{DI} 4+\mathrm{DR} 4 * \mathrm{DI} 3$

$\mathrm{SNR}=\mathrm{BK} 1 \mathrm{RB} * \mathrm{DR} 2-\mathrm{BK} 1 \mathrm{IB} * \mathrm{DI} 2+\mathrm{BI} 1 \mathrm{RB} * \mathrm{DR} 3-\mathrm{BI} 1 \mathrm{IB} * \mathrm{DI} 3$

$S N I=B K 1 R B * D I 2+B K 1 I B * D R 2+B I 1 R B * D I 3+B I 1 I B * D R 3$

$\mathrm{DEN}=\mathrm{DDR} * \mathrm{DDR}+\mathrm{DDI} * \mathrm{DDI}$

$\mathrm{SSR}=((\mathrm{SNR} * \mathrm{DDR}+\mathrm{SNI} * \mathrm{DDI}) / \mathrm{DEN}-\mathrm{BI} 1 \mathrm{~B}) / \mathrm{BK} 1 \mathrm{~B}$

$S S I=(S N I * D D R-S N R * D D I) /(B K I B * D E N)$

$\mathrm{XFACT}=\mathrm{XKR} 21 * \mathrm{DSIN}(\mathrm{XL} / 2$.

SMIMPR $=S M I M P R+8 . * X F A C T * X F A C T * S 1(J K L) * S S R / P I$

SMIMPI $=S M I M P I+8 . * X F A C T * X F A C T * S I(J K L) * S S I / P I$

DO $89 \mathrm{NR}=1$, NRT

$\mathrm{RD}=(\operatorname{REAL}(\mathrm{NR})-0.5) *(\mathrm{RDT} / \mathrm{REAL}(\mathrm{NRT}))$

IF (IS IDE. EQ. 1) THEN 
$\mathrm{RD}=\mathrm{B}+\mathrm{RD}$

ELSE

$\mathrm{KD}=\mathrm{A}-\mathrm{KD}$

END IF

$\mathrm{X} 1 \mathrm{R}=\mathrm{X} 1 * \mathrm{RD}$

$\mathrm{Y} 1 \mathrm{R}=\mathrm{Y} 1 * \mathrm{RD}$

CALL CMDBES (X1R, Y1R, ZIORR, ZIOIR, ZKORR, ZKOIR, BI1RR, BI1IR

*, BKIRR, BKIIR)

$D N R=B K 1 R R * D R 2-B K 1 I R * D I 2+B I 1 R R * D R 3-B I 1 I R * D I 3$

$\mathrm{DNI}=\mathrm{BK} 1 \mathrm{IR} * \mathrm{DR} 2+\mathrm{BK} 1 \mathrm{RR} * \mathrm{DI} 2+\mathrm{BI} 1 \mathrm{IR} * \mathrm{DR} 3+\mathrm{BI} 1 \mathrm{RR} * \mathrm{DI} 3$

$\mathrm{DFR}=(\mathrm{DNR} * \mathrm{DDR}+\mathrm{DNI} * \mathrm{DDI}) / \mathrm{DEN}$

$\mathrm{DFI}=(\mathrm{DNI} * \mathrm{DDR}-\mathrm{DNR} * \mathrm{DDI}) / \mathrm{DEN}$

DO $88 \mathrm{NZ}=1$, NZT

$\mathrm{ZD}=\mathrm{DELTAZ} * \mathrm{NZ}$

$\mathrm{XFACT} 1=4 . * \mathrm{XFACT} * \mathrm{DSIN}(\mathrm{X} * \mathrm{ZD}) * \mathrm{DSIN}(0.5 * \mathrm{X} * \mathrm{C}) * \mathrm{~S} 1(\mathrm{JKL}) / \mathrm{PI}$

$\mathrm{XFACT} 2=4 . * \mathrm{XFACT} * \mathrm{DCOS}(\mathrm{X} * \mathrm{ZD}) * \mathrm{DCOS}(0.5 * \mathrm{X} * \mathrm{C}) * \mathrm{~S} 1(\mathrm{JKL}) / \mathrm{PI}$

SMZDFR1A $(\mathrm{NZ}, \mathrm{NR})=\mathrm{SMZDFR} 1 \mathrm{~A}(\mathrm{NZ}, \mathrm{NR})+\mathrm{XFACT} 1 * \mathrm{DFR}$

SMZDFIIA $(N Z, N R)=S M Z D F I 1 A(N Z, N R)+X F A C T 1 * D F I$

SMZDFR $2 A(N Z, N R)=S M Z D F R 2 A(N Z, N R)+X F A C T 2 * D F R$

88 CONTINUE

89 CONTINUE

90 CONTINUE

$\mathrm{B} 1=\mathrm{B} 2$

$\mathrm{B} 2=\mathrm{B} 2+\mathrm{S} 2(\mathrm{JKL})$

CHECK $=($ SMAIR - RI9) $/$ SMAIR

IF (ABS (CHECK) . GT.ERR (JKL)) GO TO 30

100 CONTINUE:

DO $990 \mathrm{NZ}=1, \mathrm{NZT}$

$\mathrm{ZD}=\mathrm{DELTAZ} * \mathrm{NZ}$

SMZDFR $1=0$.

SMZDFI $1=0$.

SMZDFR2 $=0$.

SMZDFI $2=0$.

DO $120 \mathrm{NR}=1$, NRT

SMZDFR1=SMZDFR1+SMZDFR1A(NZ, NR)

SMZDFI 1=SMZDFI1+SMZDFIIA (NZ, NR)

SMZDFR2=SMZDFR2+SMZDFR2A(NZ,NR)

SMZDFI2=SMZDFI2+SMZDFI2A(NZ,NR)

120 CONTINUE

SMZDFR $1=$ SMZDFR $1 /$ NRT

SMZDF I $1=$ SMZDFI1/NRT

SMZDFR2=SMZDFR2/NRT

SMZDFI2=SMZDFI2/NRT

C

WRITE $($ LOU , *) NZ, SMZDFR1, SMZDFI1

C

WRITE $(\text { LOU }, *)^{\prime}$

', SMZDFR2, SMZDFI2

DSFR $=-1.5 *$ WUSRR * (SMZDFR $1 *$ SMZDFR2 - SMZDFI $1 *$ SMZDFI 2$) /($ SMAIR*PI $)$

DSFI $=-1.5 *$ WUSRR * (SMZDFR $1 *$ SMZDFI2+SMZDFR $2 *$ SMZDFI1) $/($ SMAIR *PI)

$135 \mathrm{Q} 6=0.0254 * 4.0 \mathrm{E}-7 * \mathrm{TRN} * \mathrm{TRN} * \mathrm{R} 3 * \mathrm{PI} * \mathrm{PI} * \mathrm{SMAIR} /(\mathrm{L} *(\mathrm{R} 2-\mathrm{R} 1)) * * 2$ 


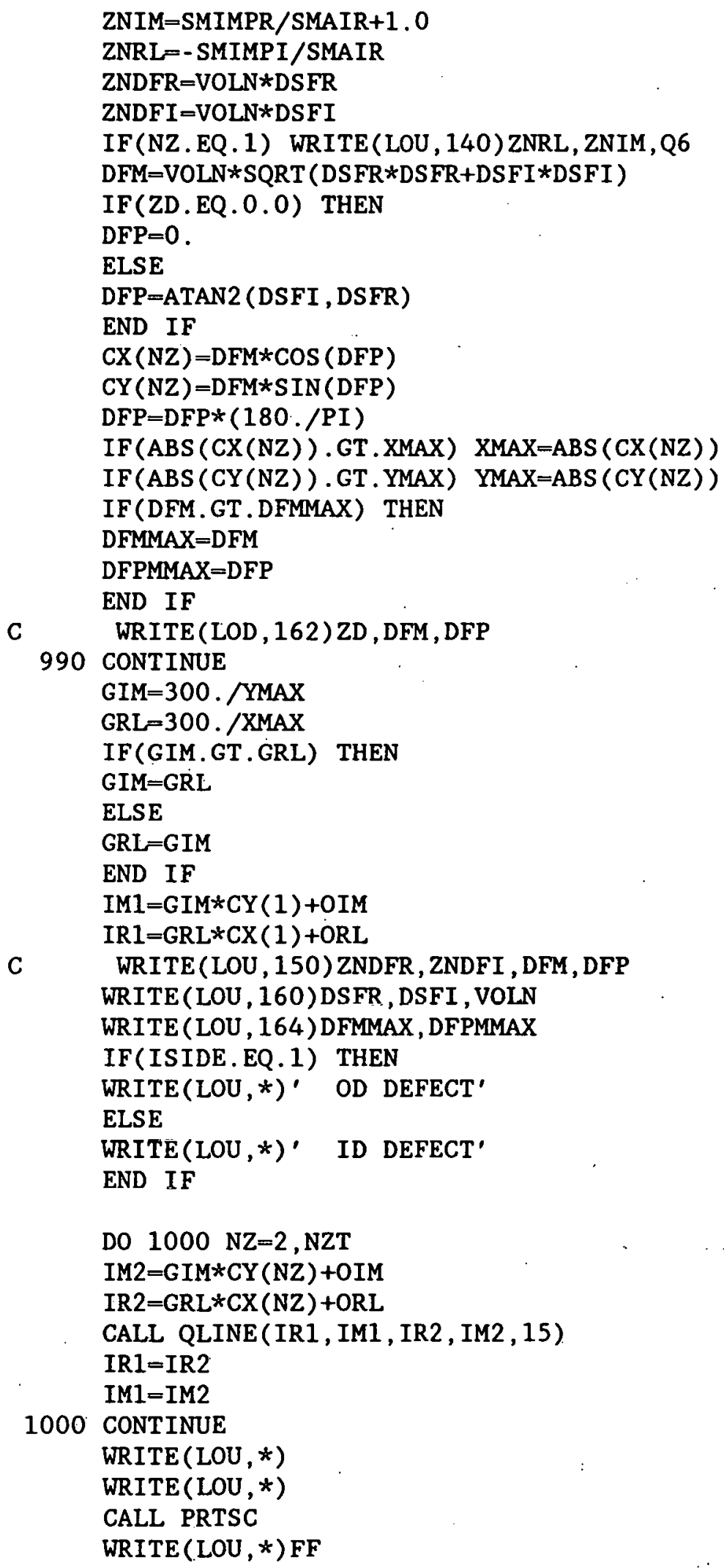


GO TO 11

140 FORMAT(' NORM IMPD: $\mathrm{RL}^{\prime}, \mathrm{F} 10.6,,^{\prime}$ IM', F'10.6, *' AIR IND', 1PE13.6)

C 150 FORMAT(' NORM CHG:RL ',OPF10.6,' IM',OPF10.6,

C *'MAG',OPF10.6,' PHA ',OPF7.2)

160 FORMAT(' NORM DSF:RL', 1PE11.4,' IM', 1PE11.4,' VOLN', IPE11.4)

161 FORMAT(' 2D MAG

162 FORMAT(F6. 3, 5X, D11.4, 5X, D11.4)

164 FORMAT(' MAXIMUM MAG ',D11.4,' PHA AT MAX MAG ',F7.2, )

1001 END 


\section{SUBROUTINES}

The following is a collection of subroutines that are used by many of the programs. Rather than list them every time with each program, they are collected here. A more detailed description of these routines is given in other reports. ${ }^{4-6}$ 


\section{Subroutine BESO(XJOR,R)}

Subroutine BESO calculates $J_{0}(r)$, the Bessel function of the first kind of order 0 . It is called by program PCBLDF.

\section{Input}

$\mathbf{R}$ The argument of the function.

\section{Output}

XJOR The function $J_{0}(r)$

\section{Listing}

SUBROUTINE BESO(XJOR,R)

C PROGRAM TO CALCULATE JO(R)

IMPLICIT REAL*8 (A-H,O-Z)

IF(R.GT. 3.0)GO TO 50

$\mathrm{Y}=\mathrm{R} * \mathrm{R} / 9.0$

$\mathrm{XJOR}=(((((.00021 * \mathrm{Y}-.0039444) * \mathrm{Y}+.0444479) * \mathrm{Y}-.3163866)$

$1 * Y+1.2656208) * Y-2.2499997) * Y+1.0$

GO TO 100

$50 \mathrm{Y}=3 / \mathrm{R}$

$\mathrm{FO}=(((((.00014476 * \mathrm{Y}-.00072805) * \mathrm{Y}+.00137237) * \mathrm{Y}=.00009512)$

$1 * Y-.00552740) * Y-.00000077) * Y+.79788456$

$\mathrm{ANG}=(((((.00013558 * \mathrm{Y}-.00029333) * \mathrm{Y}-.00054125) * \mathrm{Y}+.00262573)$

$1 * Y-.00003954) * Y-.04166397) * Y-.78539816+R$

100 RETURN

$\mathrm{XJOR}=\mathrm{F} 0 * \operatorname{COS}(\mathrm{ANG}) / \mathrm{SQRT}(\mathrm{R})$

END 
Subroutine BESELI(Q1,RJ1)

Subroutine BESEL1 calculates $J_{1}(r)$, the Bessel function of the first kind of order 1 . It is called by programs PCDSF and PCAVZSCN.

Input

Q1 The argument of the function

Output

RJ1 The function $J_{1}(r)$

\section{Listing}

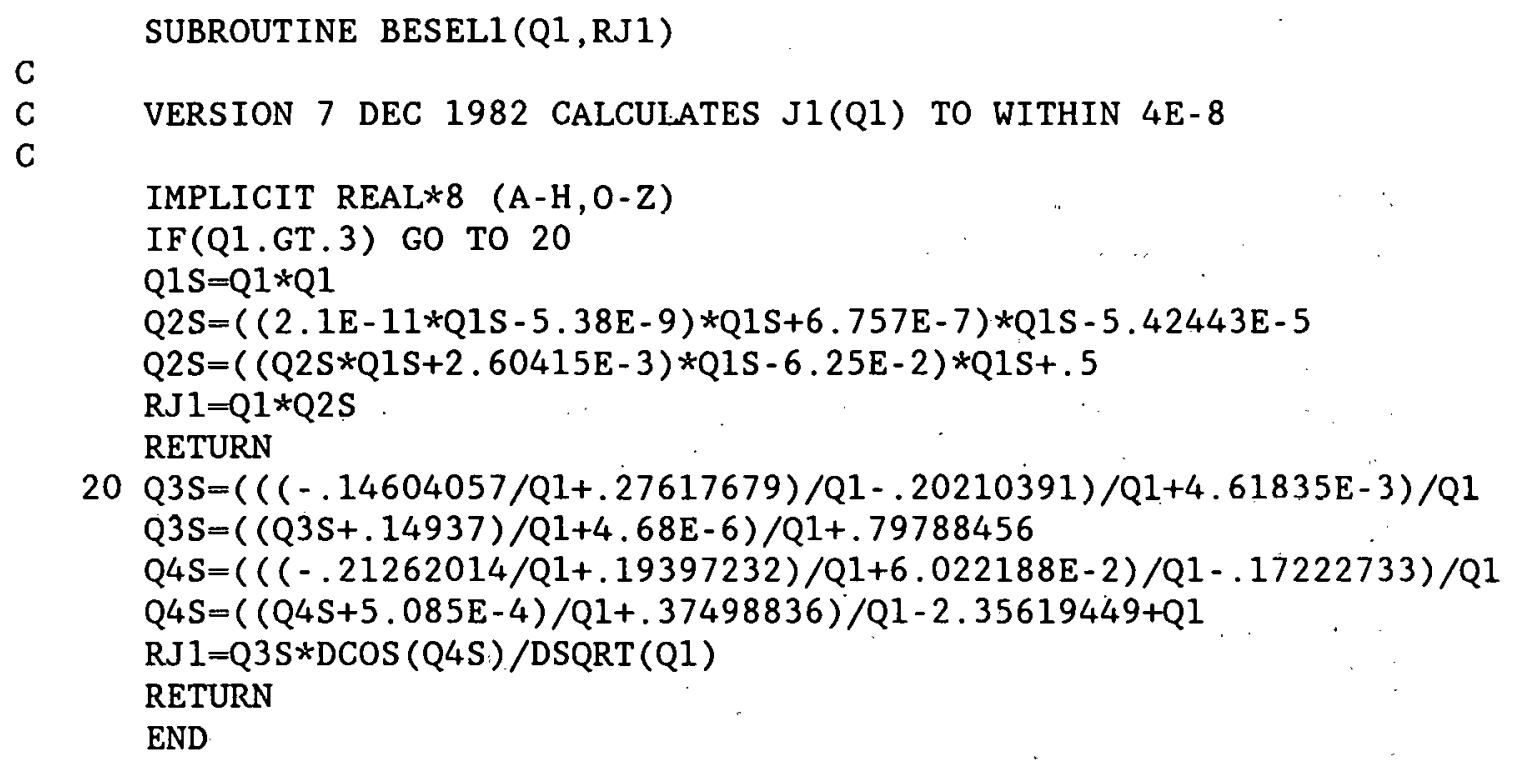


Subroutine BESI(X,XIZRO, XIONE)

Subroutine BESI calculates $I_{0}(x)$ and $I_{1}(x)$ where $I_{0}$ is the modified Bessel function of the first kind of order 0 , and $I_{1}$ is the modified Bessel function of the first kind of order 1 .

\section{Input}

$\mathrm{X} \quad$ The argument of the functions

Output

$\begin{array}{ll}\text { XKZRO } & I_{0}(\mathbf{x}) \\ \text { XKONE } & I_{1}(\mathbf{x})\end{array}$

Listing

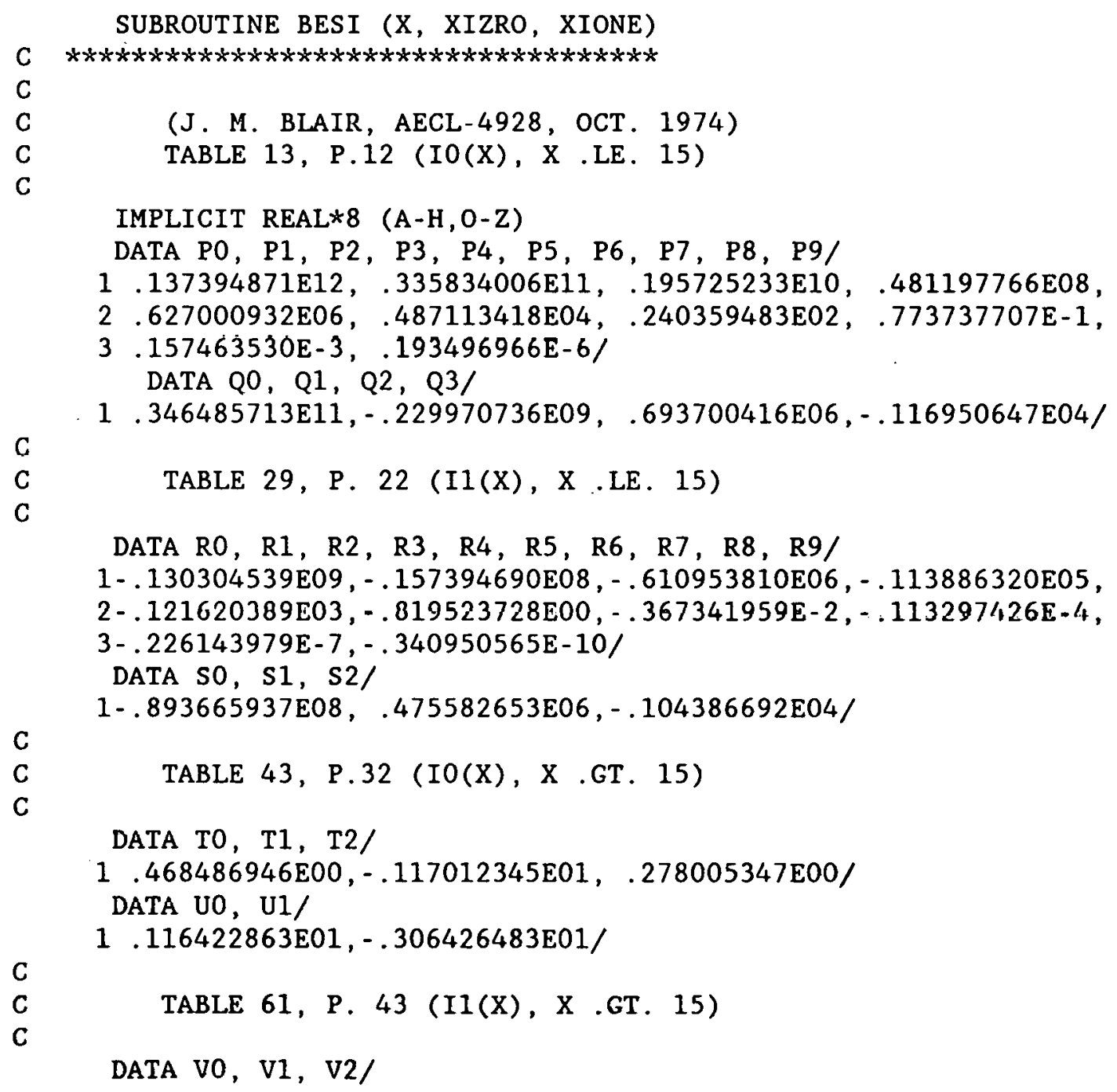




\section{2}

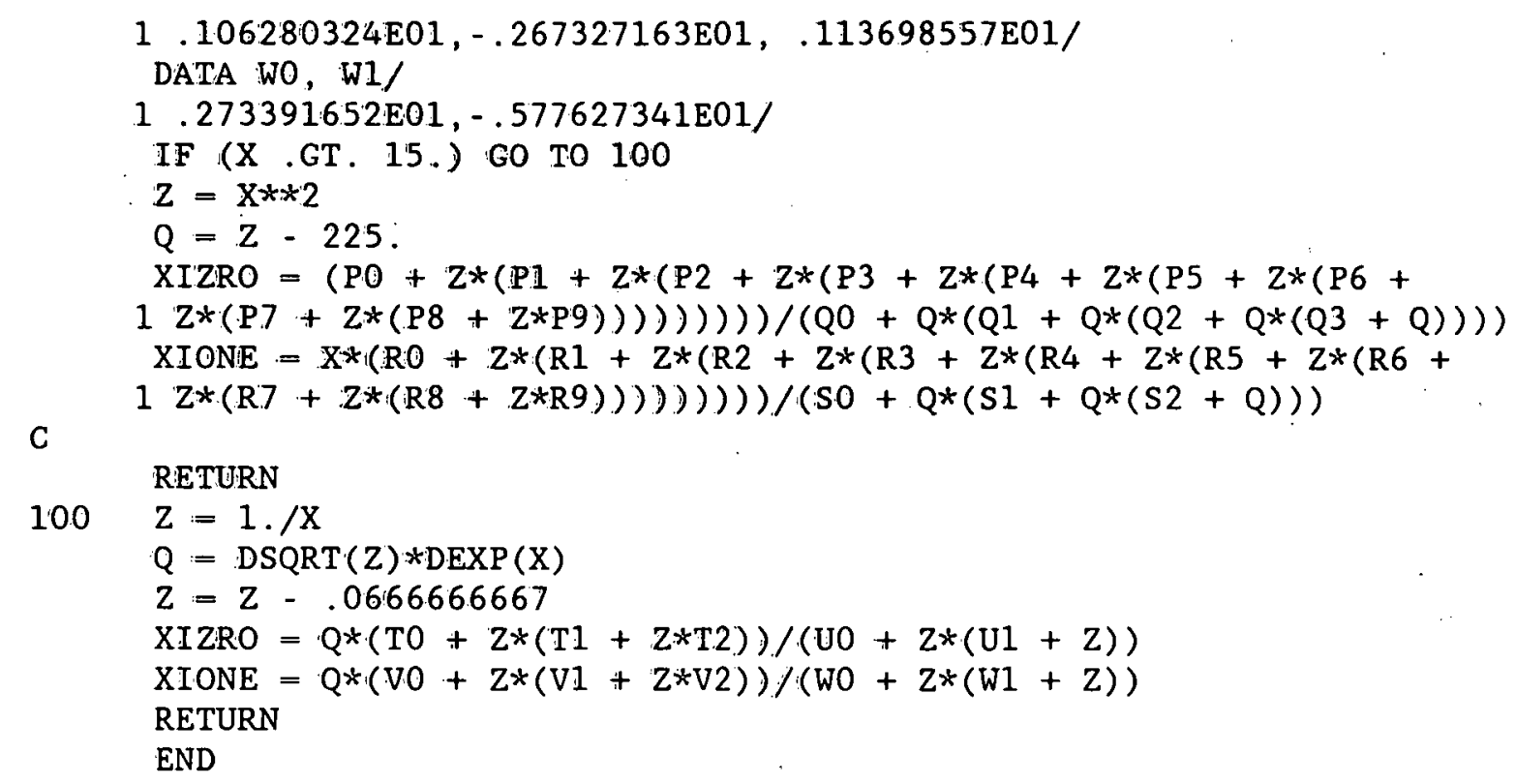




\section{Subroutine BESK( $\mathrm{X}, \mathrm{XKZRO}, \mathrm{XKONE})$}

Subroutine BESK calculates $K_{0}(x)$ and $K_{1}(x)$ where $K_{0}$ is the modified Bessel function of the second kind of order 0 , and $K_{1}$ is the modified Bessel function of the second kind of order 1 .

Input

$\mathrm{X}$ The argument of the functions

\section{Output}

$\begin{array}{ll}\text { XKZRO } & K_{0}(\mathrm{x}) \\ \text { XKONE } & K_{1}(\mathrm{x})\end{array}$

\section{Listing}

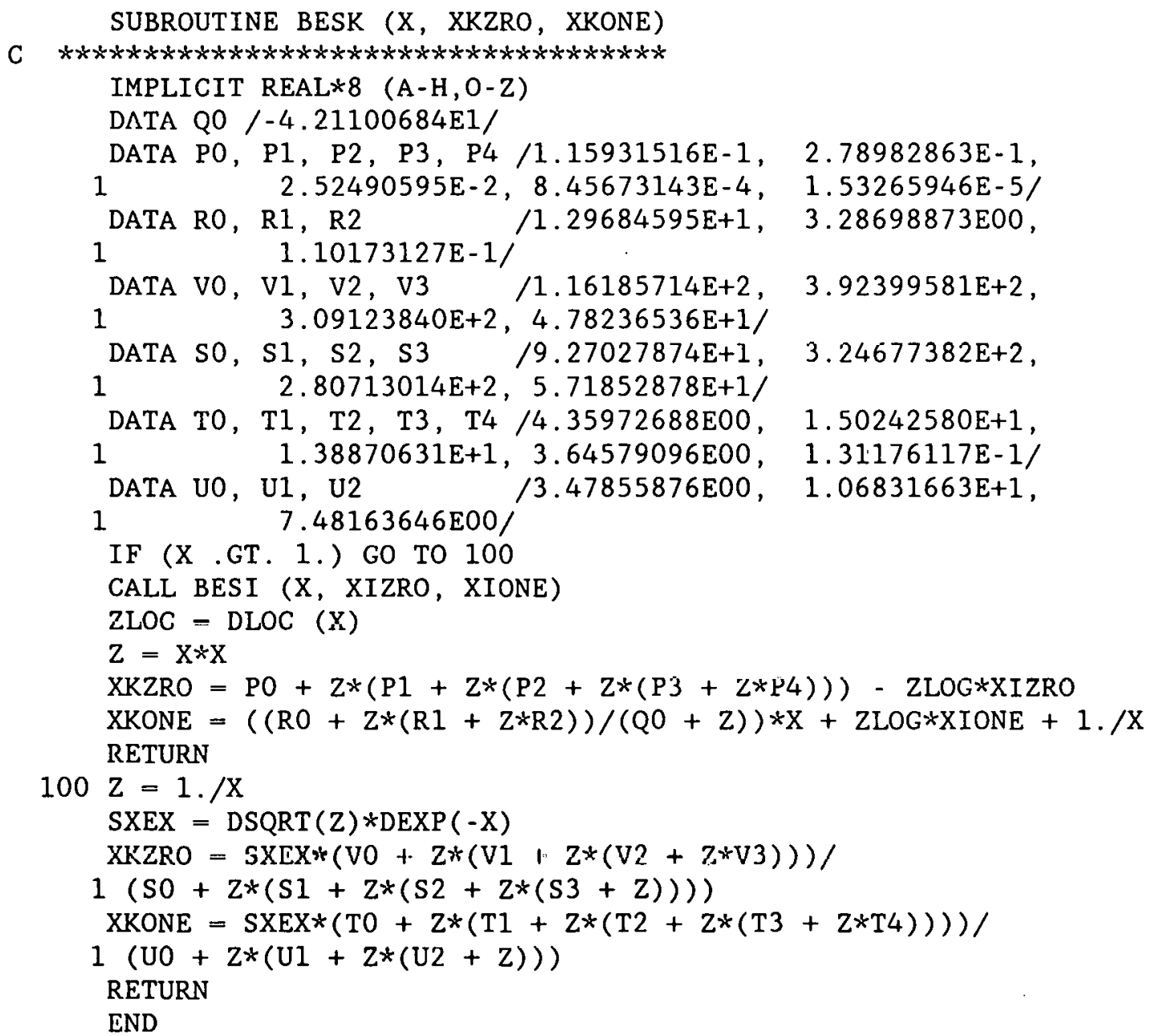


Subroutine BESSEL(XJ1, $\mathrm{X}, \mathrm{R})$

Subroutine BESSEL calculates

$$
\frac{1}{\alpha^{3}} J\left(r_{1}, 0\right)=\frac{1}{\alpha^{3}} \int_{0}^{r_{1}} \alpha r J_{1}(\alpha r) d r
$$

where $J_{1}$ is the Bessel function of the first kind of order 1 . It is called by programs PCBLDF, PCDSF, and PCAVZSCN.

\section{Input}

$$
\begin{array}{ll}
X & \alpha \text { in the above equation } \\
R & r, \text { in the above equation }
\end{array}
$$

\section{Output}

XJ1 The right side of the above equation

\section{Listing}

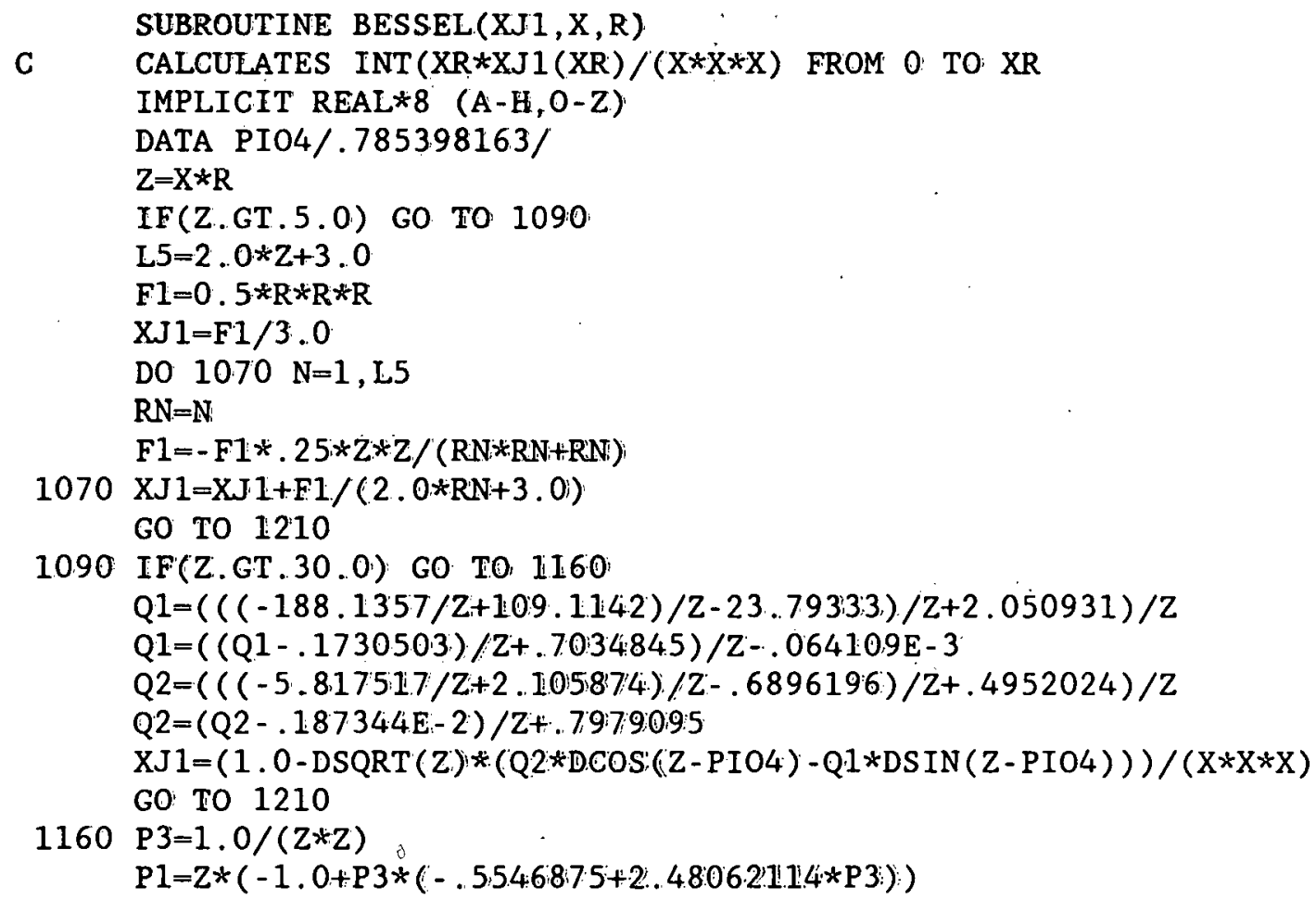


$\mathrm{P} 2=.875+\mathrm{P} 3 *(-.93457031+8.98975114 * \mathrm{P} 3)$

$\mathrm{XJ} 1=1.0+.79788456 *(\mathrm{P} 1 * \mathrm{DCOS}(\mathrm{Z}-.78539816)$

$8+\mathrm{P} 2 * \operatorname{DSIN}(Z-.78539816)) / \mathrm{DSQRT}(\mathrm{Z})$

$\mathrm{XJ} 1=\mathrm{XJ} 1 /(\mathrm{X} * \mathrm{X} * \mathrm{X})$

1210 RETURN

END 
Subroutine CMDBES (X,Y,ZIOR,ZIOI,ZKOR,ZKOI, BIIR, BIII, BKIR,BKII)

Subroutine CMDBES calculates the following four quantities: $z I_{0}(z)$, $z K_{0}(z), I_{1}(z)$, and $K_{1}(z)$, where $z$ is a complex number and where $I_{0}$ is the modified Bessel function of the first kind of order $0, K_{0}$ is the modified Bessel function of the second kind of order $0, I_{1}$ is the modified Bessel function of the first kind of order 1 , and $K_{0}$ is the modified Bessel function of the second kind of order 1 .

\section{Input}

$\begin{array}{ll}\mathrm{X} & \operatorname{Re}(z) \\ \mathrm{Y} & \operatorname{Im}(z)\end{array}$

output

$\begin{array}{ll}\text { ZIOI } & \operatorname{Im}\left[z I_{0}(z)\right] \\ \text { ZIOR } & \operatorname{Re}\left[z I_{0}(z)\right] \\ \text { ZKOI } & \operatorname{Im}\left[z K_{0}(z)\right] \\ \text { ZKOR } & \operatorname{Re}\left[z K_{0}(z)\right] \\ \text { BI1I } & \operatorname{Im}\left[I_{1}(z)\right] \\ \text { BI1R } & \operatorname{Re}\left[I_{i}(z)\right] \\ \text { BK1I } & \operatorname{Im}\left[K_{1}(z)\right] \\ \text { BK1R } & \operatorname{Re}\left[K_{1}(z)\right]\end{array}$

\section{Listing}

SUBROUTINE CMDBES (X, Y, ZIOR, ZIOI , ZKOR, ZKOI , BI 1R , BI1I , BK1R, BK1I)

C
C
C
C
C
C
C
C

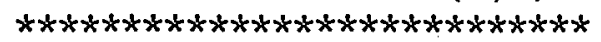

FOR R.LE. 8 USE RATIONAL APPROXIMATION FOR KO(Z) AND K1(Z) AND BACKWARD RECURRENCE FOR IO(Z) AND II $(Z)$

CALL $\operatorname{COMKB}(X, Y, Z K O R, Z K O I, B K 1 R, B K 1 I)$

CALL CMI (X, Y, ZIOR, ZIOI, BI1R, BIII)

GO TO 125 


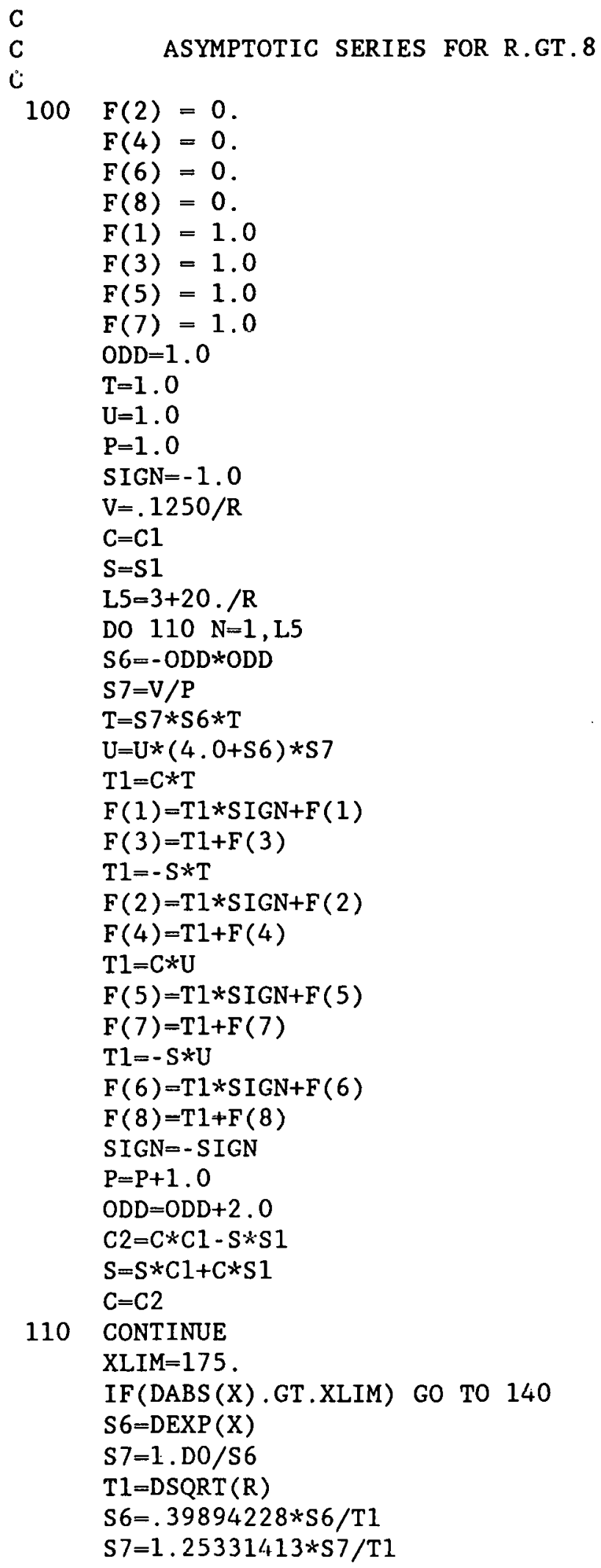


C

C

C

THE ABOVE FACTORS ARE 1/DSQRT(2*PI) AND DSQRT.(PI/2)

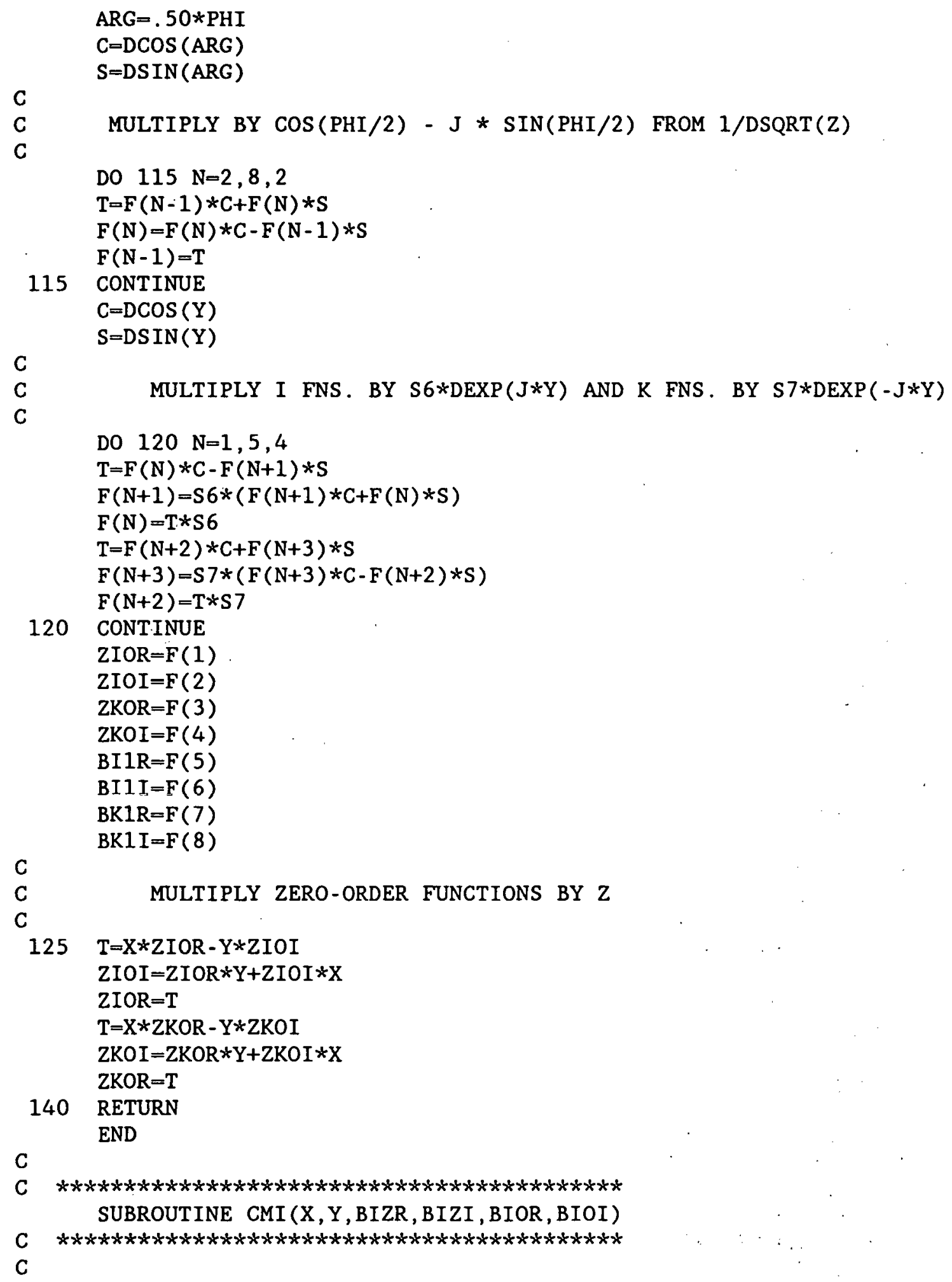

C

C

$125 \mathrm{~T}=\mathrm{X} * \mathrm{ZIOR}-\mathrm{Y} * \mathrm{ZIOI}$

$\mathrm{ZIOI}=\mathrm{ZIOR} * \mathrm{Y}+\mathrm{ZIOI} * \mathrm{X}$

$\mathrm{ZIOR}=\mathrm{T}$

$\mathrm{T}=\mathrm{X} * \mathrm{ZKOR}-\mathrm{Y} * \mathrm{ZKOI}$

$Z K O I=Z K O R * Y+Z K O I * X$

$\mathrm{ZKOR}=\mathrm{T}$

140 RETURN

END

C

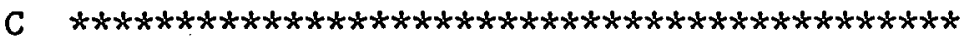

SUBROUTINE CMI (X,Y,BIZR, BIZI, BIOR, BIOI)

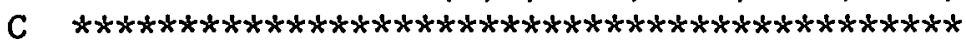

$\mathrm{C}$ 
C

C

C

C

C

C

C

C

C

C

C

C

C

C

C

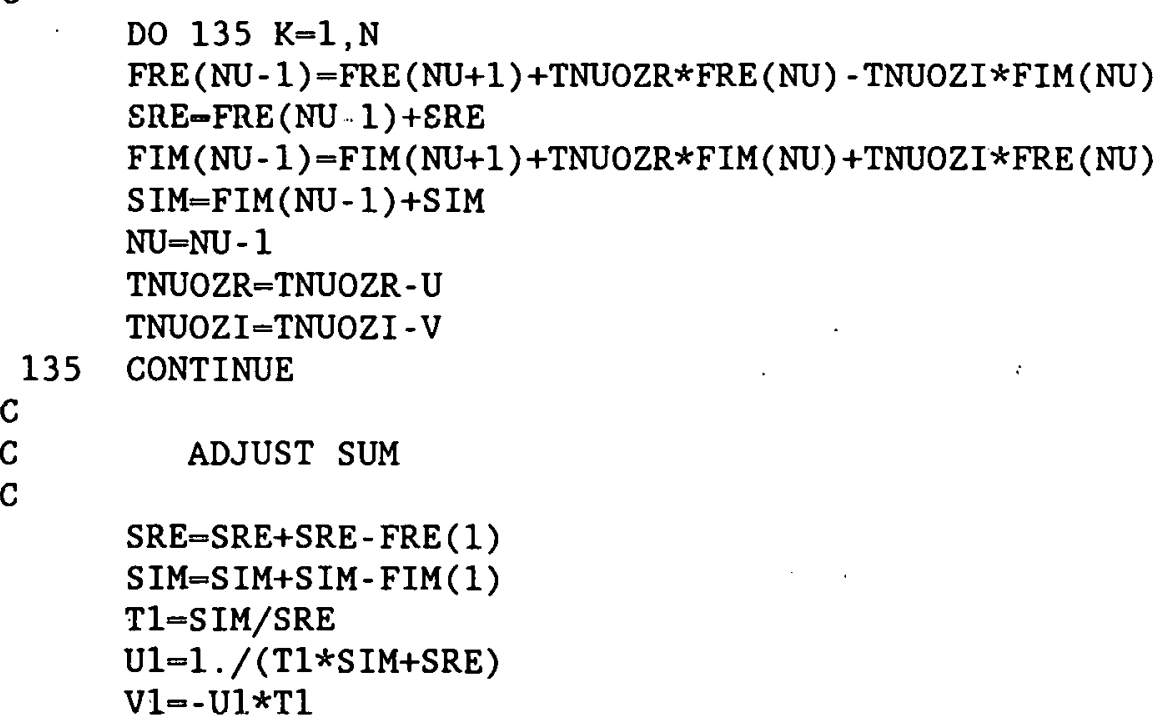


C

C

C

NORMALIZE

$\operatorname{EX}=\operatorname{DEXP}(X)$

$S Y=D S I N(Y)$

$C Y=D C O S(Y)$

FANRE $=E X *(C Y * U 1-S Y * V 1)$

FANIM $=E X *(C Y * V 1+S Y * U 1)$

BIZR $=$ FANRE $*$ FRE (1) - FANIM $*$ FIM (1)

$B I Z I=$ FANRE $*$ FIM $(1)+$ FANIM*FRE (1)

$B I O R=F A N R E * F R E(2)-$ FANIM*FIM( 2$)$

337 RETURN

BIOI=FANRE $*$ FIM $(2)+$ FANIM $*$ FRE $(2)$

END

C $* * * * * * * * * * * * * * * * * * * * * * * * * * * * * * * * * * * * * * * * * * * * * * * * * * * *$

SUBROUTINE COMKB (X, Y, BKZR, BKZI, BKOR, BKOI)

C

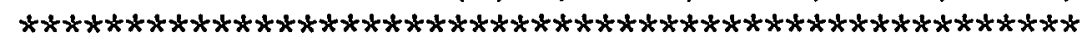

COMPUTES THE REAL \& IMAGINARY PARTS OF THE MODIFIED BESSEL FUNCTIONS $K O(Z) \& K 1(Z)$, WHERE $Z=X+I * Y$, BY THE METHOD OF Y.L. LUKE, THE SPECIAL FUNCTIONS AND THEIR APPROXIMATIONS, VOL.2, PG. 229.

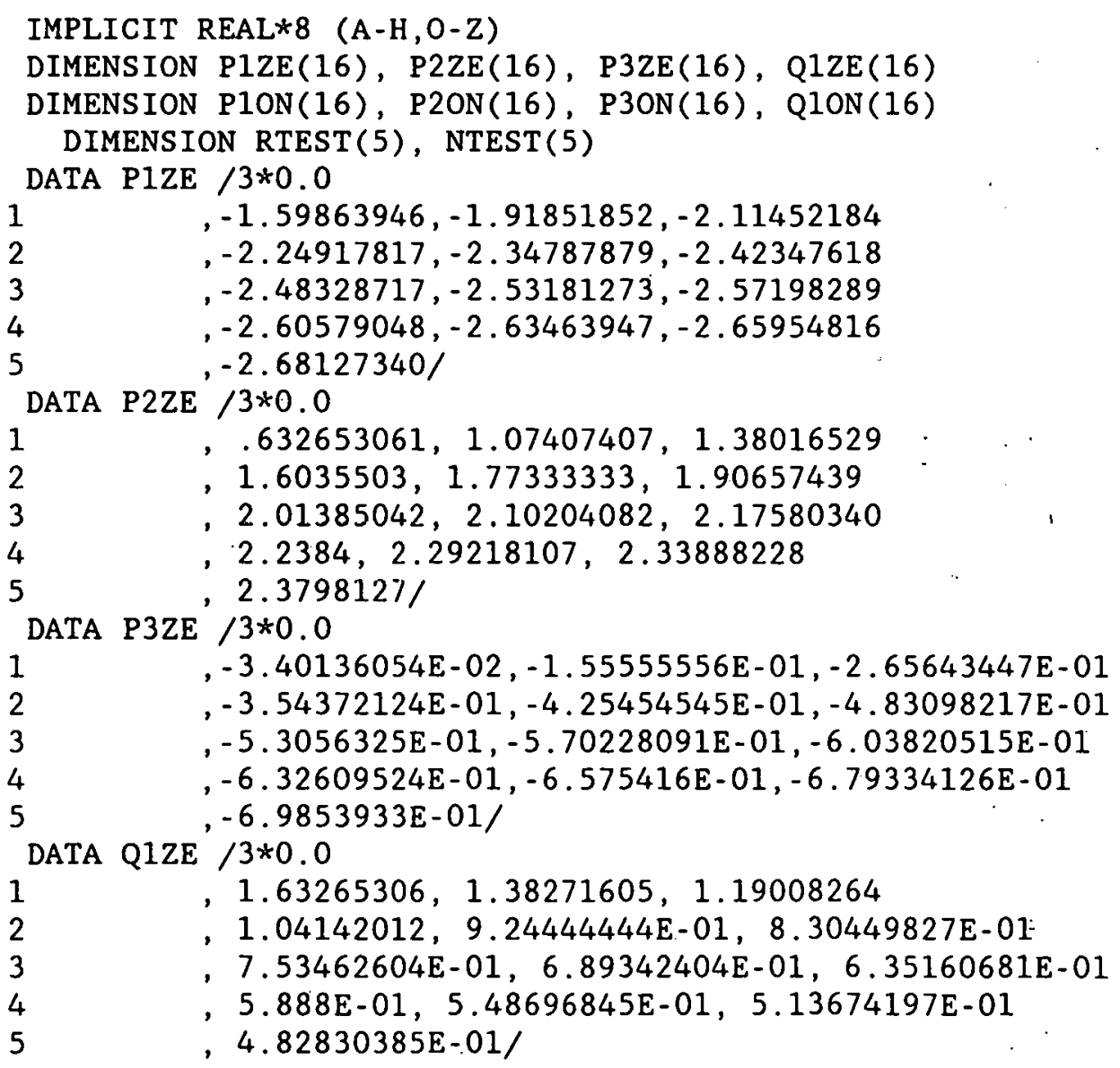




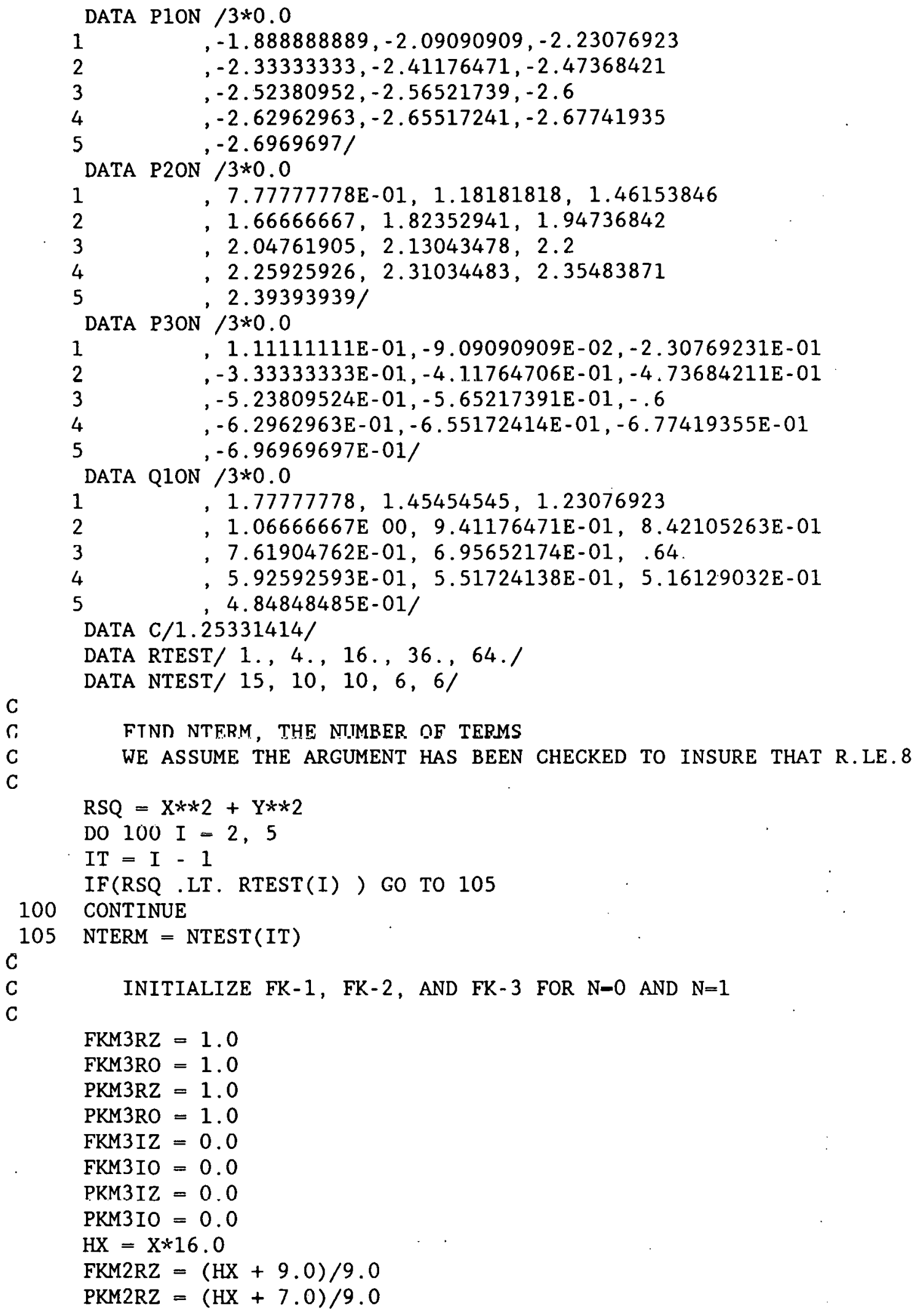


$F X=X * 3.20$

FKM2RO $=F X+1.0$

PKM2RO $=$ FKM2RO +1.20

FKM2IO $=\mathrm{Y} * 3.20$

PKM2IO $=$ FKM2IO

$\mathrm{HY}=\mathrm{Y} * 16.0$

FKM2IZ $=\mathrm{HY} / 9.0$

PKM2IZ = FKM2IZ

HYS $=H Y * * 2$

$\mathrm{T}=\mathrm{HX}+25.0$

FKM1RZ $=(\mathrm{HX} * \mathrm{~T}+75.0-\mathrm{HYS}) / 75.0$

$\mathrm{FKM1IZ}=\mathrm{HY} *(\mathrm{HX}+\mathrm{T}) / 75.0$

$\mathrm{T}=\mathrm{HX}+23.0$

PKMIRZ $=(H X * T+43.0-$ HYS $) / 75.0$

$\mathrm{PKM1IZ}=\mathrm{HY} *(\mathrm{HX}+\mathrm{T}) / 75.0$

$\mathrm{T}=\mathrm{HX}+21.0$

FKM1RO $=(H X * T+35.0-H Y S) / 35.0$

FKM1IO $=\mathrm{HY} *(\mathrm{HX}+\mathrm{T}) / 35.0$

$\mathrm{T}=\mathrm{HX}+27.0$

PKMIRO $=\left(\mathrm{HX}_{1}^{* \mathrm{~T}}+131.0-\mathrm{HYS}\right) / 35.0$

C

PKM1IO $=\mathrm{HY} *(\mathrm{HX}+\mathrm{T}) / 35.0$

C

C

BEGIN RECURRENCE

DO $110 \mathrm{~K}=3$, NTERM

$\mathrm{KP} 1=\mathrm{K}+1$

C

CALCULATIONS OF FKRZ, FKIZ, PKRZ, AND PKIZ FOR $\mathrm{N}=0$

$\mathrm{P} 1=\mathrm{P} 1 \mathrm{ZE}(\mathrm{KP} 1)$

$\mathrm{P} 2=\mathrm{P} 2 \mathrm{ZE}(\mathrm{KP} 1)$

$\mathrm{P} 3=\mathrm{P} 3 Z \mathrm{E}(\mathrm{KP} 1)$

$\mathrm{Q} 1=\mathrm{Q} 12 \mathrm{E}(\mathrm{KP} 1)$

$\mathrm{HX}=\mathrm{Q} 1 * \mathrm{X}$

$\mathrm{HY}=\mathrm{Q} I * \mathrm{Y}$

$\mathrm{T} 1=\mathrm{FKM} 1 \mathrm{RZ}+\mathrm{FKM} 2 \mathrm{RZ}$

$\mathrm{T} 2=\mathrm{FKM} 1 \mathrm{IZ}+\mathrm{FKM} 2 \mathrm{IZ}$

FKRZ $=\mathrm{HX} * \mathrm{~T} 1-\mathrm{P} 1 * \mathrm{FKM} 1 \mathrm{RZ}-\mathrm{P} 2 * \mathrm{FKM} 2 \mathrm{RZ}-\mathrm{HY} * \mathrm{~T} 2-\mathrm{P} 3 * \mathrm{FKM} 3 \mathrm{RZ}$

FKIZ $=\mathrm{HX} * \mathrm{~T} 2-\mathrm{P} 1 * F K M 1 I Z-\mathrm{P} 2 * \mathrm{FKM} 2 \mathrm{IZ}+\mathrm{HY} * \mathrm{~T} 1-\mathrm{P} 3 * \mathrm{FKM} 3 \mathrm{IZ}$

FKM3RZ $=$ FKM2RZ

FKM2RZ $=$ FKM1RZ

FKMIRZ $=$ FKRZ

FKM3IZ = FKM2IZ

FKM2IZ = FKM1IZ

FKM1IZ = FKIZ

$\mathrm{T} 1=\mathrm{PKM} 1 \mathrm{RZ}+\mathrm{PKM} 2 \mathrm{RZ}$

$\mathrm{T} 2=\mathrm{PKM} 1 \mathrm{IZ}+\mathrm{PKM} 2 \mathrm{IZ}$

$\mathrm{PKRZ}=\mathrm{HX} * \mathrm{~T} 1-\mathrm{P} 1 * \mathrm{PKM} 1 \mathrm{RZ}-\mathrm{P} 2 * \mathrm{PKM} 2 \mathrm{RZ}-\mathrm{HY} * \mathrm{~T} 2-\mathrm{P} 3 * \mathrm{PKM} 3 \mathrm{RZ}$

$\mathrm{PKIZ}=\mathrm{HX} * \mathrm{~T} 2-\mathrm{P} 1 * \mathrm{PKM} 1 \mathrm{IZ}-\mathrm{P} 2 * \mathrm{PKM} 2 \mathrm{IZ}+\mathrm{HY} * \mathrm{~T} 1-\mathrm{P} 3 * \mathrm{PKM} 3 \mathrm{IZ}$

PKM3RZ = PKM2RZ

PKM2RZ = PKM1RZ 


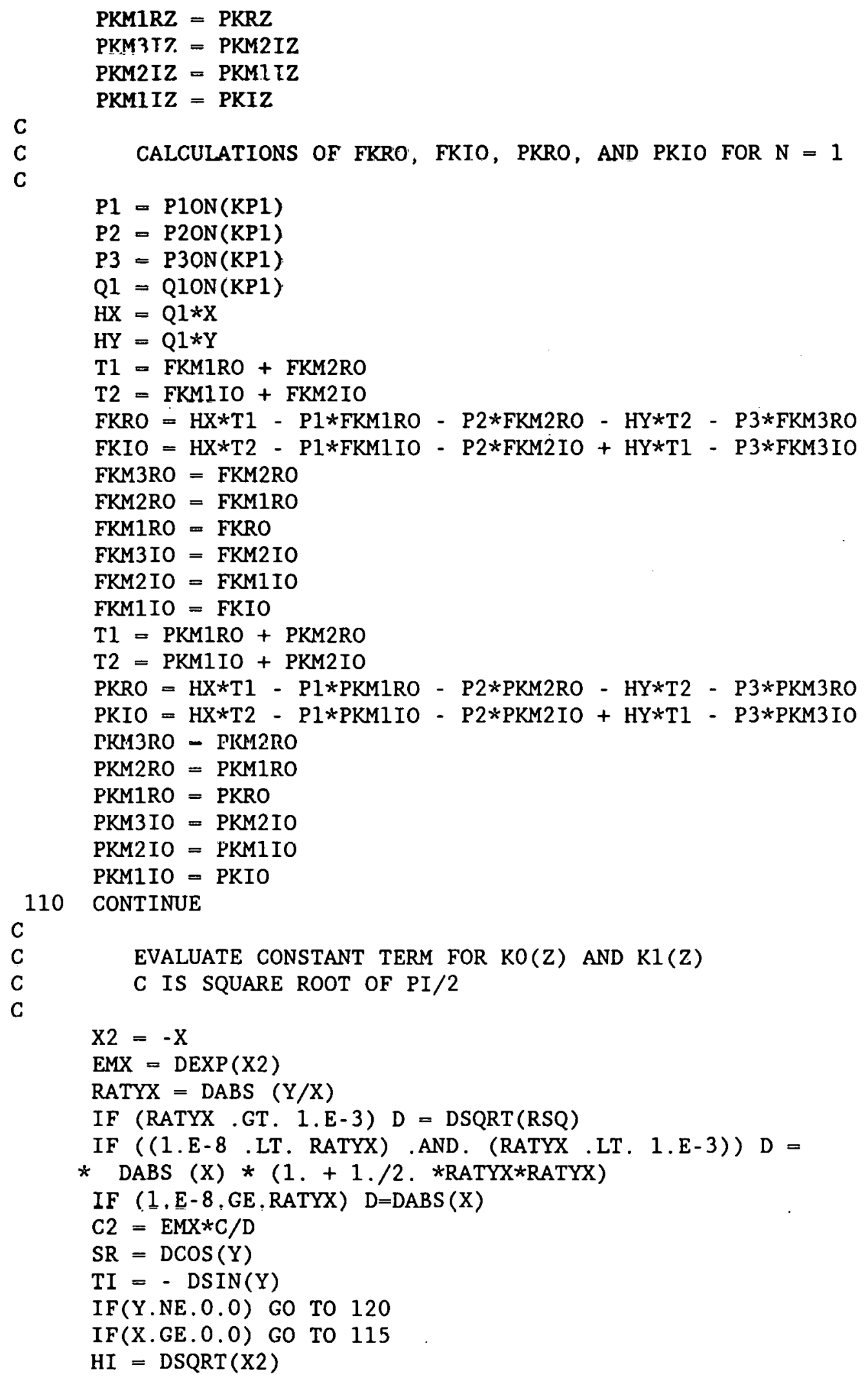




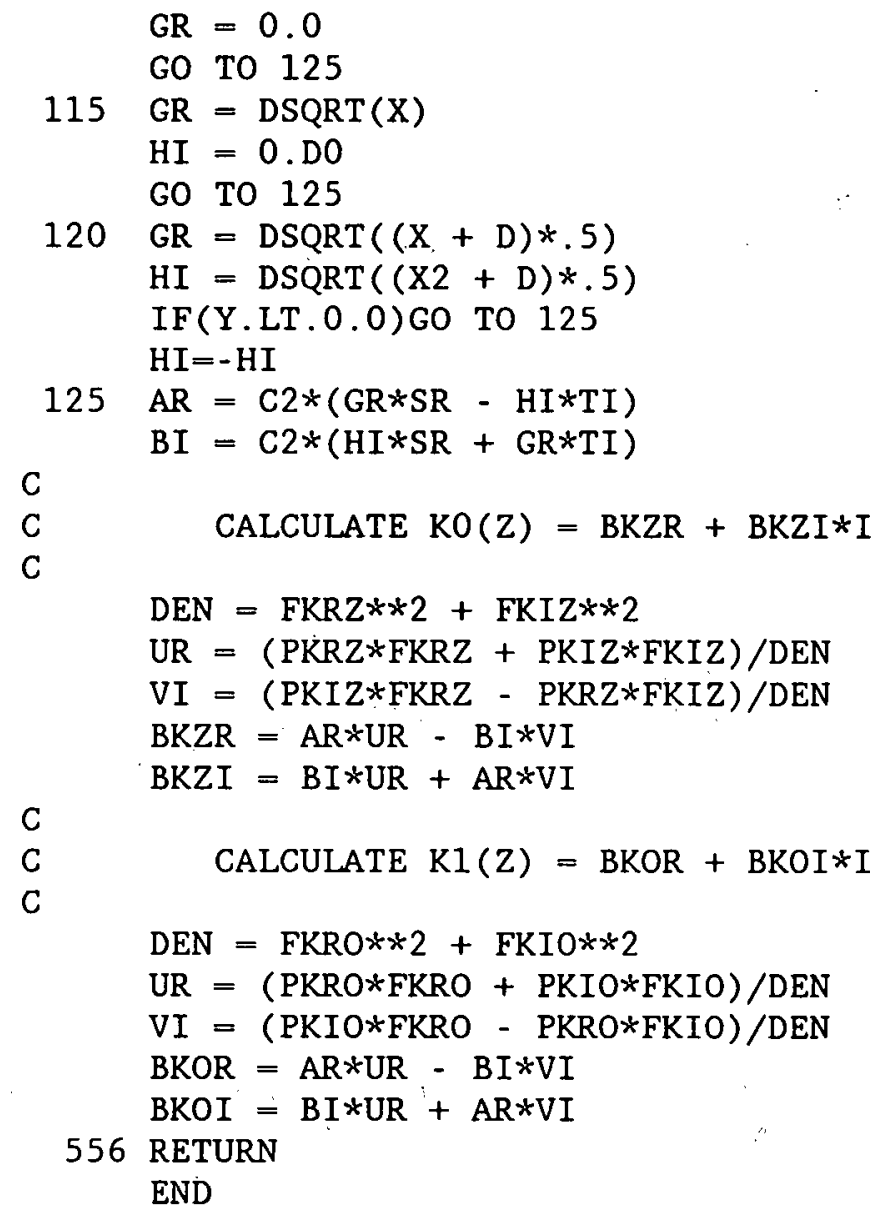

C

C

CALCULATE $\mathrm{KI}(\mathrm{Z})=\mathrm{BKOR}+\mathrm{BKOI} \times \mathrm{I}$

$\mathrm{DEN}=\mathrm{FKRO} * * 2+\mathrm{FKIO} * * 2$

$\mathrm{UR}=(\mathrm{PKRO} * \mathrm{FKRO}+\mathrm{PKIO} * \mathrm{FKIO}) / \mathrm{DEN}$

$V I=(P K I O * F K R O-P K R O * F K I O) / D E N$

$\mathrm{BKOR}=\mathrm{AR} * \mathrm{UR}-\mathrm{BI} * \mathrm{VI}$

$\mathrm{BKOI}=\mathrm{BI} * \mathrm{UR}+\mathrm{AR} * \mathrm{VI}$

556 RETURN

END 
Subroutine GRID

Subroutine GRID draws a grid on the screen.

\section{Listing}

SUBROUTINE GRID

CALL QLINE $(30,0,630,0,7)$

CALL QLINE $(30,50,630,50,7)$

CALL QLINE $(30,100,630,100,7)$

CALL QLINE $(30,150,630,150,7)$

CALL QLINE $(30,200,630,200,7)$

CALL QLINE $(30,250,630,250,7)$

CALL QLINE $(30,300,630,300,7)$

CALL QLINE $(30,349,630,349,7)$

CALL QLINE $(30,0,30,349,7)$

CALL QLINE $(80,0,80,349,7)$

CAI.L QLINE $(130,0,130,349,7)$

CALL QLINE $(180,0,180,349,7)$

CALL QLINE $(230,0,230,349,7)$

CALL QLINE $(280,0,280,349,7)$

CALL QLINE $(330,0,330,349,7)$

CALL QLINE $(380,0,380,349,7)$

CALL QLINE $(430,0,430,349,7)$

CALL QLINE $(480,0,480,349,7)$

CALL QLINE $(530,0,530,349,7)$

CALL QLINE $(580,0,580,349,7)$

EALL QLINE $(630,0,630,349,7)$

RETURN

END 
Subroutine IJBSSL(A, R1, R2, I, J)

Subroutine IJBSSL calculates

$$
\frac{1}{\alpha^{3}} I\left(r_{2}, r_{1}\right)=\frac{1}{\alpha^{3}} \int_{r_{1}}^{r_{2}} \alpha r I_{1}(\alpha r) d r
$$

and

$$
\frac{1}{\alpha^{3}} J\left(r_{2}, r_{1}\right)=\frac{1}{\alpha^{3}} \int_{r_{1}}^{r_{2}} \alpha r J_{1}(\alpha r) d r
$$

where $I_{1}$ is the modified Bessel function of the first kind of order 1 , and $J$, is the Bessel function of the first kind of order 1 .

\section{Input}
A
$\alpha$ in the above equations
R1 $r_{1}$ in the above equations
R2 $r_{2}$ in the above equations

\section{Output}

I The right side of the first equation above

$\mathrm{J}$ The right side of the second equation above

\section{Listing}

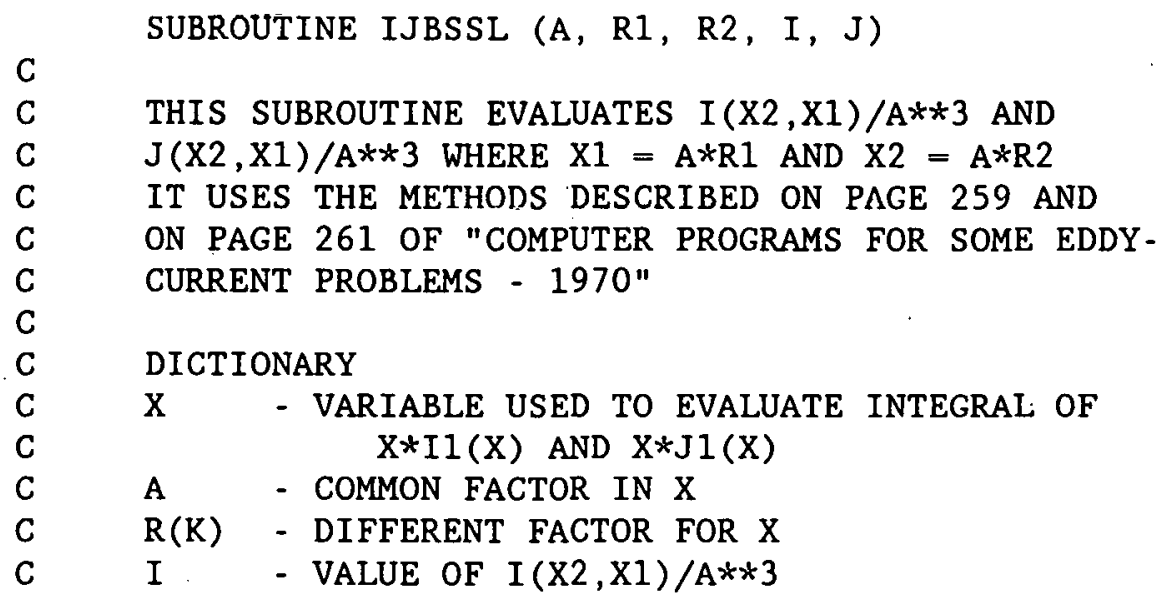




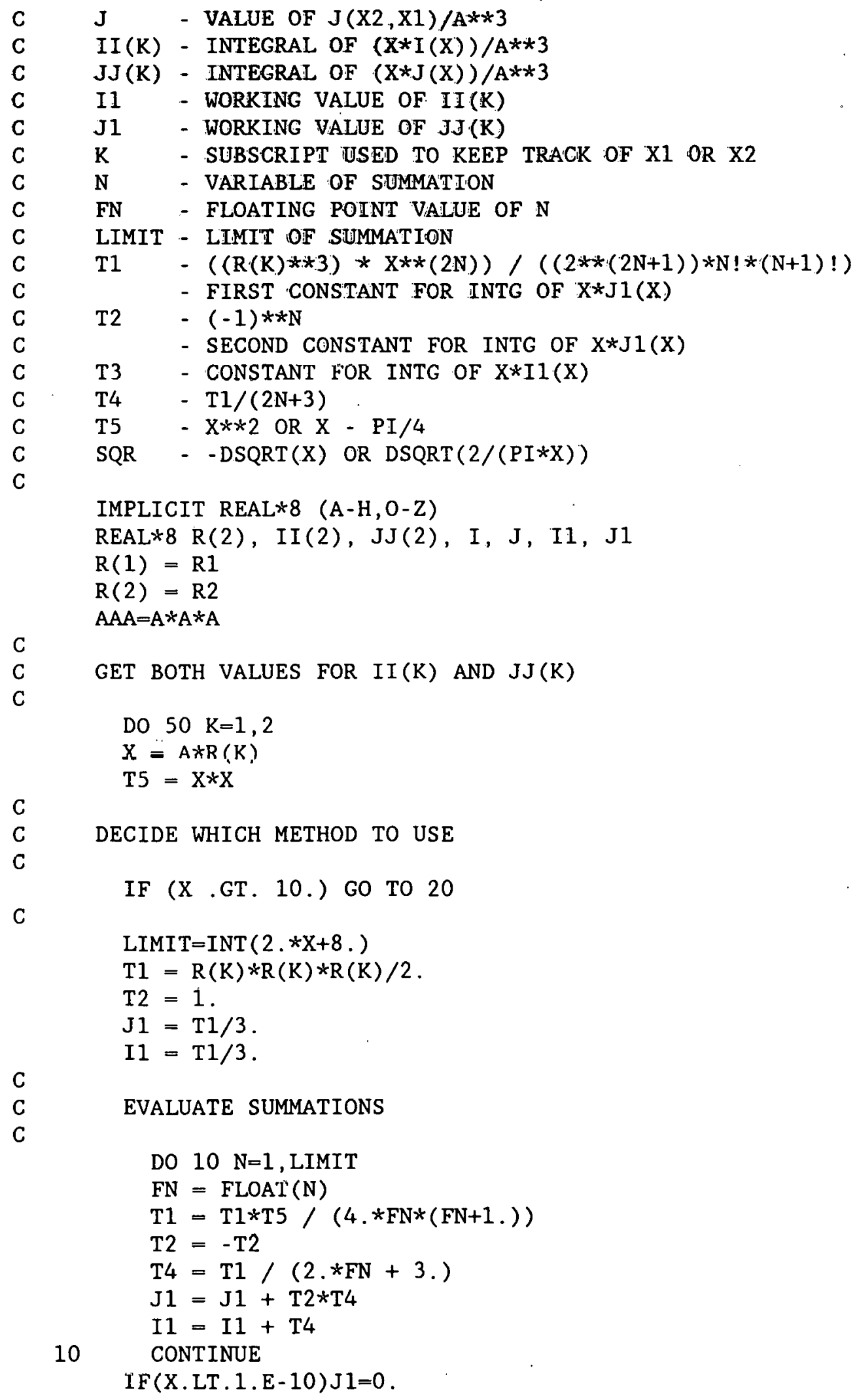




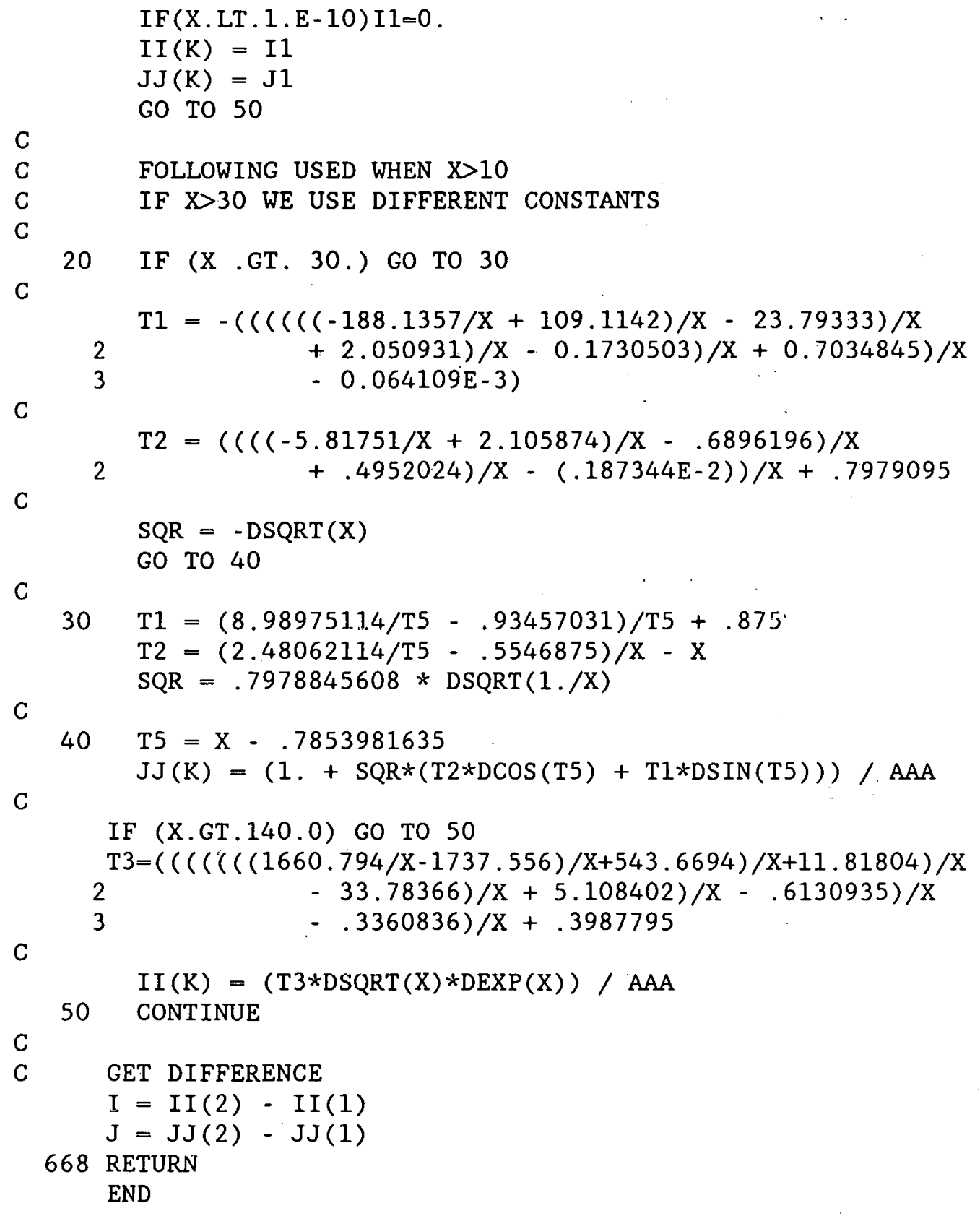


Subroutine $\operatorname{KJBSSL}(A, R 1, R 2, K, J)$

Subroutine KJBSSL calculates

$$
\frac{1}{\alpha^{3}} K\left(r_{2}, r_{1}\right)=\frac{1}{\alpha^{3}} \int_{r_{1}}^{r_{2}} \alpha r K_{1}(\alpha r) d r
$$

and

$$
\frac{1}{\alpha^{3}} J\left(r_{2}, r_{1}\right)=\frac{1}{\alpha^{3}} \int_{r_{1}}^{r_{2}} \alpha r . J_{1}(\alpha r) d r
$$

where $K_{1}$ is the modified Bessel function of the second kind of order 1 , and $J_{1}$ is the Bessel function of the first kind of order 1 .

$\underline{\text { Input }}$
A
$\alpha$ in the above equations
R1
$r_{1}$ in the above equations
R2
$r_{2}$ in the above equations

\section{Output}

$$
\begin{array}{ll}
K & \text { The right side of the first equation above } \\
J & \text { The right side of the second equation above }
\end{array}
$$

\section{Listing}

$\mathrm{C}$

C THIS SUBROUTINE EVALUATES $\mathrm{K}(\mathrm{X} 2, \mathrm{X} 1) /(\mathrm{A} * * 3)$ AND

C $\mathrm{J}(\mathrm{X} 2, \mathrm{X} 1) /(\mathrm{A} * * 3)$ WHERE X1 $=\mathrm{A} * \mathrm{R}(1)$ AND $\mathrm{X} 2=\mathrm{A} * \mathrm{R}(2)$

C IT USES THE METHODS DESCRIBED ON PAGE 259 AND ON

C PAGE 263 OF "COMPUTER PROGRAMS FOR SOME EDDY-

C CURRENT PROBLEMS - 1970"

C

C. DTCTTONARY

C $\mathrm{X}$ - VARIABLE USED TO EVALUATE INTEGRAL

C $\mathrm{X} * \mathrm{~K} 1(\mathrm{X})$ AND $\mathrm{X} * \mathrm{~J} 1(\mathrm{X})$

C A - COMMON FACTOR IN X

C $R(I)$ - FACTOR FOR DIFFERENT VALUES OF $X$

C $\mathrm{K}$ - VALUE OF $\mathrm{K}(\mathrm{X} 2, \mathrm{X} 1) /(\mathrm{A} * * 3)$ 


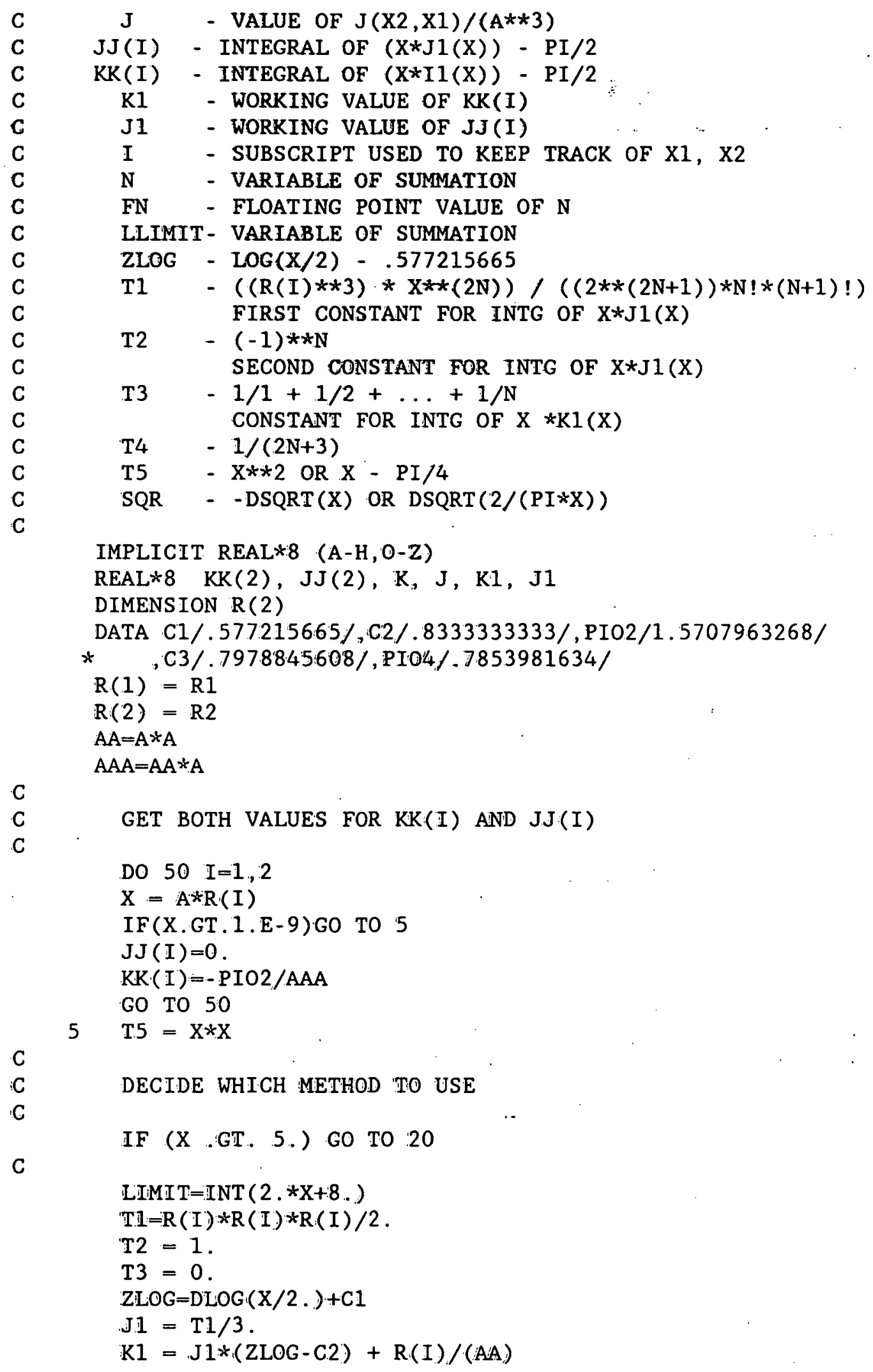


C

C

C

10

$\mathrm{KK}(\mathrm{I})=\mathrm{K} 1$ - PIO2 $/ \mathrm{AAA}$

$\mathrm{JJ}(\mathrm{I})=\mathrm{J} 1$

GO TO 50

C

C

C

C

C

C 2

FOLLOWING IS USED WHEN $X>5$

IF $X>30$ WE USE DIFFERENT CONSTANTS FOR $X * J 1(X)$

20 IF (X.GT. 30.) GO TO 30

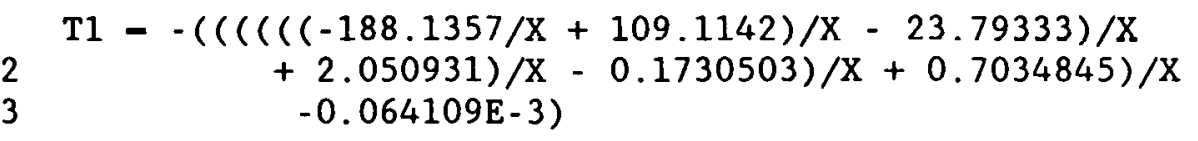

$\mathrm{T} 2=((((-5.817517 / \mathrm{X}+2.105874) / \mathrm{X}-.6896196) / \mathrm{X}$

C

$\mathrm{SQR}=-\mathrm{DSQRT}(\mathrm{X})$

GO TO 40

C

C

40 T5 $=\mathrm{X}-\mathrm{PIO} 4$

$$
\mathrm{JJ}(\mathrm{I})-(1 .+\mathrm{SQR} *(\mathrm{~T} 2 * \mathrm{DCOS}(\mathrm{T} 5)+\mathrm{T} 1 * \mathrm{DSIN}(\mathrm{T} 5))) / \mathrm{AAA}
$$

C

C

$\mathrm{C}$

C

C

IF $X$ IS GREATER THAN 77, WE EXPERIENCE UNDERFLOW

IN CALCULATING $\mathrm{KK}(\mathrm{I})$ AND SO SET $\mathrm{KK}(\mathrm{I})$ TO 0.0

IF (X .GT. 77.0) GO TO 45

$\mathrm{T} 3=((((.79898397 / \mathrm{X}-1.1768576) / \mathrm{X}+0.9157142 .1) / \mathrm{X}$

2 $-.67491295) / \mathrm{X}+1.0958276) / \mathrm{X}+1.2533263$

$K K(I)=-\operatorname{DSQRT}(\mathrm{X}) * \mathrm{DEXP}(-\mathrm{X}) * \mathrm{~T} 3 / \mathrm{AAA}$

GO TO 50

$45 \mathrm{KK}(\mathrm{I})=0.0$

50 CONTINUE

$$
\mathrm{K}=\mathrm{KK}(2)-\mathrm{KK}(1)
$$$$
\mathrm{J}=\mathrm{JJ}(2)-\mathrm{JJ}(1)
$$ 
RETURN

END 
Subroutine PCLKUP(DEPTH, RHSMAG, LHSPHA)

Subroutine PCLKUP searches through a lookup table created by program PCBLDF to find the depth and magnitude of an integral corresponding to the phase which it is given as input. It is called by programs PCINV and PCRTSCAN.

Input

LHSPHA The phase of the integral

Output

DEPTH The depth at which the integral has this phase

RHSMAG The magnitude of the integral when it has this phase

\section{Listing}

SUBROUTINE PCLKUP (DEPTH, RHSMAG, LHSPHA)

IMPLICIT REAL*8(A-H,O-Z)

REAL $* 8$ LHSPHA

DATA PI/3.141592653/,LOU/8/,LOE/40/

OPEN (LOE , FILE $=$ ' ASPHAJO. DAT' , STATUS $={ }^{\prime} \mathrm{OLD}^{\prime}$ )

C OPEN (LOE, FILE $=$ 'ADPHAF. DAT' , STATUS $={ }^{\prime}$ OLD' $)$

RHSPHAO $=0$.

1120 READ (LOE , *, END=1380) Z, RHSMAG , RHSPHA

DPH=ABS (ABS (RHSPHA) - A $\ddot{B} S$ (RHSPHAU))

$D M G=$ RHSMAG - RHSMAGO

RLMR $=A B S(A B S($ RHSPHA $)-A B S($ LHSPHA $))$

$\mathrm{DZ}=\mathrm{Z}-\mathrm{ZO}$

IF (RHSPHAO.EQ.0.) GO TO 1180

IF (RHSPHA.GE. LHSPHA) THEN

IF (RHSPHAO . LT . LHSPHA) THEN

$A F=R L M R / D P H$

DEPTH $=Z-D Z * A F$

RHSMAG $=$ RHSMAG $-D M G * A F$

GO TO 1400

END IF

ELSE

IF (RHSPHAO .GE. LHSPHA) THEN

$A F=R L M R / D P H$

DEPTH $=Z-D Z * A F$

RHSMAG $=$ RHSMAG $-D M G * A F$

GO TO 1400

END IF

END IF

$1180 \mathrm{ZO}=\mathrm{Z}$

RHSMAGO $=$ RHSMAG

RHS $P H A 0=$ RHS $P H A$ 


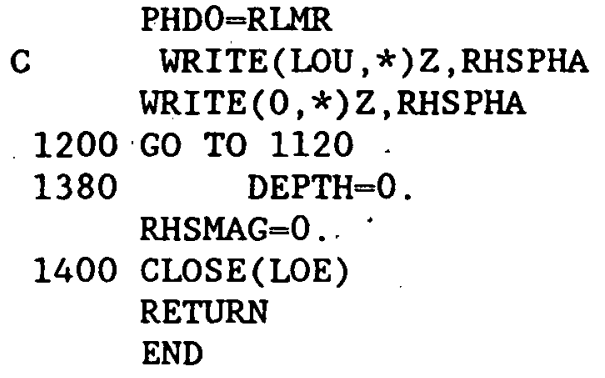


Subrout ine RFLKUP(DEPTH, RHSMAG, LHSPHA)

Subroutine RFLKUP searches through a lookup table created by program RFBLDF to find the depth and magnitude of an integral corresponding to the phase which it is given as input. It is called by program RFINV.

Input

LHSPHA The phase of the integral

Output

DEPTH The depth at which the integral has phase LHSPHA

RHSMAG The magnitude of the integral when it has phase IHSPHA

\section{Listing}

SUBROUTINE RFLKUP(DEPTH, RHSMAG, LHSPHA)

IMPLICIT REAL*8(A-H,O-Z)

REAL $* 8$ LHSPHA

DATA PI/3.141592653/,LOU/8/,LOE/40/

OPEN (LOE , FILE $=$ ' RF25P. 500' , STATUS $=$ ' OLD' $)$

RHSPHAO $=0$.

$1140 \operatorname{READ}$ (LOE , *, END=1280) Z, RHSMAG , RHSPHA

$D P H=A B S$ (ABS (RHSPHA) - ABS (RHSPHAO))

DMG=RHSMAG - RHSMAGO

$K L M K=A B S(A B S$ (RHS PHA) $-A B S$ (LHSPHA) )

$\mathrm{DZ}=\mathrm{Z}-\mathrm{ZO}$

IF (RHSPHAO .EQ. O.) GO TO 1180

IF (RHSPHA.GE. LHSPHA) THEN

IF (RHSPHAO . LT . LHSPHA) THEN

$A F=R L M R / D P H$

$\mathrm{DEPTH}=\mathrm{Z}-\mathrm{DZ} * \mathrm{AF}$

RHSMAG $=$ RHSMAG $-D M G * A F$

GO TO $1 / 00$

END IF

ELSE

IF (RHSPHAO .GE. LHSPHA) THEN

$\mathrm{AF}=\mathrm{RLMR} / \mathrm{DPH}$

DEPTH $=Z-D Z * A F$

RHSMAG $=$ RHSMAG - DMG *AF

GO TO 1400

END IF

END IF

$1180 \mathrm{ZO}=\mathrm{Z}$

RHSMAG0 =RHSMAG

RHGFILA0-RUIGFILA

PHDO = RLMR

$\operatorname{WRITE}(0, *) Z$, RHSPHA 
1200 GO TO 1140

$1280 \mathrm{DEPTH}=0$.

RHSMAG $=0$.

1400 CLOSE (LOE) RETURN

END 
Data File REF.DAT

The following data file is required to run some of the reflection coil programs in this report.

\begin{tabular}{|c|c|c|c|c|c|c|c|c|c|c|c|c|c|c|c|}
\hline 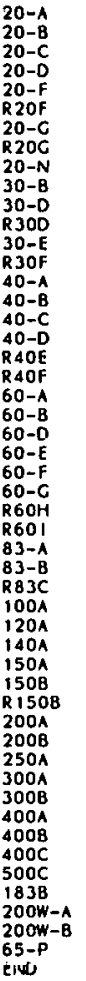 & $\begin{array}{l}0.0200 \\
0.0200 \\
0.0200 \\
0.0200 \\
0.0200 \\
0.0200 \\
0.0200 \\
0.0200 \\
0.0200 \\
0.0300 \\
0.0300 \\
0.0300 \\
0.0300 \\
0.0300 \\
0.0400 \\
0.0400 \\
0.0400 \\
0.0400 \\
0.0400 \\
0.0400 \\
0.0600 \\
0.0600 \\
0.0600 \\
0.0600 \\
0.0600 \\
0.0600 \\
0.0600 \\
0.0600 \\
0.0832 \\
0.0832 \\
0.0830 \\
0.1000 \\
0.1200 \\
0.1400 \\
0.1500 \\
0.1500 \\
0.1500 \\
0.2000 \\
0.2000 \\
0.2500 \\
0.3000 \\
0.3000 \\
0.4000 \\
0.4000 \\
0.4000 \\
0.5000 \\
0.1830 \\
0.9000 \\
\end{array}$ & 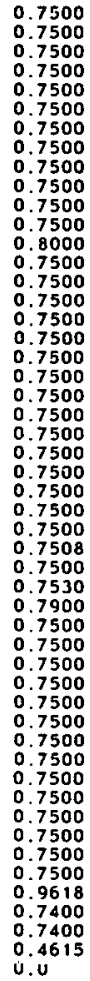 & $\begin{array}{l}1.2500 \\
1.2500 \\
1.2500 \\
1.2500 \\
1.2500 \\
1.2500 \\
1.2500 \\
1.2500 \\
1.2500 \\
1.2500 \\
1.2000 \\
1.2500 \\
1.2500 \\
1.2500 \\
1.2500 \\
1.2500 \\
1.2500 \\
1.2500 \\
1.2500 \\
1.2500 \\
1.2500 \\
1.2500 \\
1.2500 \\
1.2500 \\
1.2500 \\
1.2490 \\
1.2500 \\
1.2530 \\
1.2100 \\
1.2500 \\
1.2500 \\
1.2500 \\
1.2500 \\
1.2500 \\
1.2500 \\
1.2500 \\
1.2500 \\
1.2500 \\
1.2500 \\
1.2500 \\
1.2500 \\
1.2500 \\
1.2500 \\
1.0382 \\
1.2600 \\
1.2600 \\
1.5385 \\
1.0\end{array}$ & $\begin{array}{l}5000 \\
9000 \\
.9000 \\
.9000 \\
.6000 \\
.6000 \\
.6000 \\
.6000 \\
.6000 \\
.6000 \\
.5000 \\
5000 \\
.5000 \\
.5000 \\
.6000 \\
.3000 \\
.6000 \\
.3000 \\
.4000 \\
4000 \\
.3000 \\
.3000 \\
4000 \\
.3000 \\
.3000 \\
.3000 \\
.3000 \\
.3000 \\
.3604 \\
.3600 \\
.3614 \\
.4200 \\
.3000 \\
.3930 \\
.4000 \\
.3600 \\
.3600 \\
.6000 \\
.4000 \\
.6000 \\
.6000 \\
.4000 \\
.6000 \\
.6000 \\
.4000 \\
.4000 \\
.3825 \\
.5000 \\
.5000 \\
.1538 \\
.0\end{array}$ & $\begin{array}{l}0.3 \\
0.3 \\
0.3 \\
0.3 \\
0.3 \\
0.3 \\
0.3 \\
0.3 \\
0.3 \\
0.3 \\
0.7 \\
0.0 \\
0.0 \\
0.3\end{array}$ & $\begin{array}{l}0.700 \\
0.700 \\
0.690 \\
0.700 \\
0.700 \\
0.700 \\
0.700 \\
0.700 \\
0.700\end{array}$ & $\begin{array}{l}0.1 \\
0.1 \\
0.2 \\
0.1 \\
0.2 \\
0.2 \\
0.0 \\
0.2 \\
0.2 \\
0.0 \\
0.0 \\
0.1 \\
0.0 \\
0.0 \\
0.0\end{array}$ & $\begin{array}{l}0.00 \\
0.00 \\
0.00 \\
0.00 \\
0.00 \\
0.00 \\
0.00 \\
0.00 \\
0.00 \\
0.00 \\
0.00 \\
0.00 \\
0.00 \\
0.00 \\
0.00 \\
0.00 \\
0.00 \\
0.00 \\
0.00 \\
0.00 \\
0.00 \\
0.00 \\
0.00 \\
0.00 \\
0.00 \\
0.00 \\
0.00 \\
0.00 \\
0.00 \\
0.00 \\
0.00 \\
0.00 \\
0.00 \\
0.00\end{array}$ & $\begin{array}{l}0 . \\
0 . \\
0 . \\
0 . \\
0 . \\
0 . \\
0 . \\
0 . \\
0 . \\
0 . \\
0 . \\
0 . \\
0 . \\
0 . \\
0 . \\
0 . \\
0 . \\
0 . \\
0 .\end{array}$ & $\begin{array}{r}3 \\
6 \\
2 \\
2 \\
0 \\
0 \\
3 \\
3 \\
0 \\
34 \\
10 \\
10 \\
0 \\
4 \\
5 \\
15 \\
84 \\
9 \\
1 \\
3\end{array}$ & $\begin{array}{l}7.30 \\
10.1 \\
4.60 \\
7.5 \\
5.0 \\
3.23 \\
3.4 \\
3.00 \\
5.30 \\
5.98 \\
5.80 \\
4.15 \\
0.0\end{array}$ & $\begin{array}{r}13 . \\
128 . \\
24 \\
23 \\
2 . \\
2 . \\
24 \\
26 \\
0 . \\
74 \\
62 \\
62 \\
2 . \\
9 . \\
205 \\
79 \\
659 \\
114 \\
11 . \\
140 .\end{array}$ & & $\begin{array}{r}10 \\
2 \\
6 \\
9 \\
11 \\
37 \\
9 \\
3 \\
6 \\
24 \\
25 \\
17 \\
6 \\
4 \\
10 \\
10\end{array}$ & $\begin{array}{r}65 \\
72 \\
25 \\
25 \\
66 \\
54 \\
8 \\
144 \\
112 \\
112 \\
21 \\
108 \\
264 \\
240 \\
510 \\
154 \\
46 \\
75 \\
450 \\
427\end{array}$ \\
\hline
\end{tabular}




\section{REFERENCES}

1. M. L. Burrows, A Theory of Eddy Current Flaw Detection, University Microfilms, Inc., Ann Arbor, Michigan, 1964.

2. C. V. Dodd, W. E. Deeds, J. W. Luquire, and W. G. Spoeri, Union Carbide Corporation Nuclear Division, Oak Ridge National Laboratory, Some Eddy-Current Problems and Their Integral Solutions, ORNL-4384, April 1969.*

3. C. V. Dodd, C. C. Cheng, and W. E. Deeds, "Induction Coils Coaxial with an Arbitrary Number of Cylindrical Conductors," J. Appl. Phys. 45(2), 638-47.†

4. C. V. Dodd, C. C. Cheng, C. W. Nestor, and R. B. Hofstra, Union Carbide Corporation Nuclear Division, Oak Ridge National Laboratory, Design of. Induction Probes for Measurement of Level of Liquid Metals, ORNL-TM-4175, May 1973*.

5. W. A. Simpson, C. V. Dodd, J. W. Luquire, and W. G. Spoeri, Union Carbide Corporation Nuclear Division, Oak Ridge National Laboratory, Computer Programs for Some Eddy-Current Problems - 1970, ORNL-TM-3295, June $1971^{*}$.

6. C. W. Nestor Jr., C. V. Dodd, and W. E. Deeds, Union Carbide Corporation Nuclear Division, Oak Ridge National Laboratory, Analysis and Computer Programs for Eddy Current Coils Concentric with Multiple Cylindrical Conductors, ORNL-5220, July 1979.*

*Available for purchase from National Technical Information Service, Springfield, Virginia 22161.

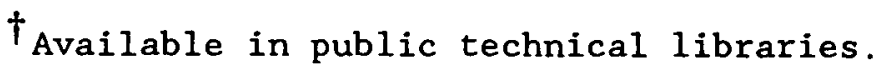


NUREG/CR - 5553

ORNL/TM- 11505

Dist. Category RF

INTERNAL DISTRIBUTION

1. W. R. Corwin

2-6. L. D. Chitwood

7-11. W. E. Deeds

12-16. C. V. Dodd

17. R. W. McClung

18. D. J. McGuire

19. R. K. Nanstad

20-24. J. R. Pate

25. J. G. Purett

26. C. E. Pugh
27. W. A. Simpson

28. G. M. Slaughter

29. J. R. Weir

30-32. M\&C Records Office

33. ORNL Patent Section

34. Central Research Library

35. Document Reference Section

36-37. Laboratory Records Department

38. Laboratory Records (RC)

39. Nuclear Safety Information

Center

EXTERNAL DISTRIBUTION

40. NRC, OFFICE OF NUCLEAR REGULATORY RESEARCH, Washington, DC 20555

G. A. Arlotto

J. Muscara

C. Z. Serpan

41. ELECTRIC POWER RESEARCH INSTITUTE, P.0. BOX 217097,

Charlotte, NC 28221

Mike Elmo

Kenji Kryzwosz

42. BATTELLE PACIFIC NORTHWEST LABORATORIES, P.O. BOX 999, Richland, WA 99352

Bob Ferris

43. ROCKY FLATS PLANT, P.O. Box 464, Golden, CO 80402 Bruce Allen

44. 23 Greensburg Strcet, Delmont Borough, PA 15626 Steve D. Brown 
45: DOE, Oak Ridge Operations Office, Oak Ridge, TN 37831-8600 Office of Assistant Manager for Energy Research and Development

46-47. DOE, Office of Scientific and Technical Information, P.0. Box 62, Oak Ridge, TN 37831

48-297. Given distribution as shown in category RF (NTIS-10) 


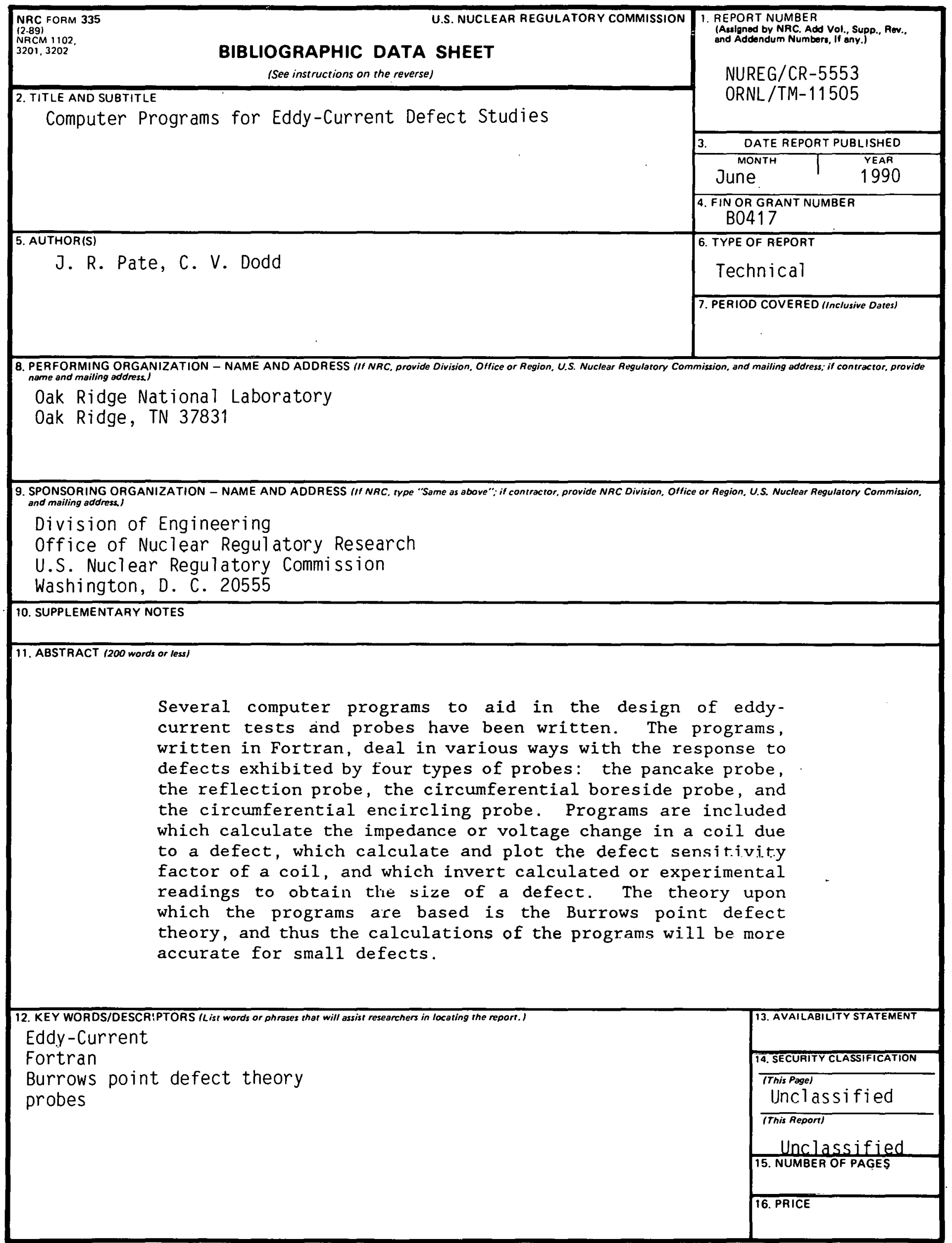


UNITED STATES

NUCLEAR REGULATORY COMMISSION

WASHINGTON, D.C. 20555

OFFICIAL BUSINESS

PENALTY FOR PRIVATE USE, $\$ 300$
SPECIAL FOUATH.CLASS RATE POSTAGE 6 FEES PAID

USNRC

PERMIT No. G-67 